\title{
The Breeding Biology and Habitat Relationships of the Yellowhead
}

Graeme Peter Elliott

Submitted for the degree of Doctor of Philosophy in Zoology at Victoria University of Wellington, New Zealand. 


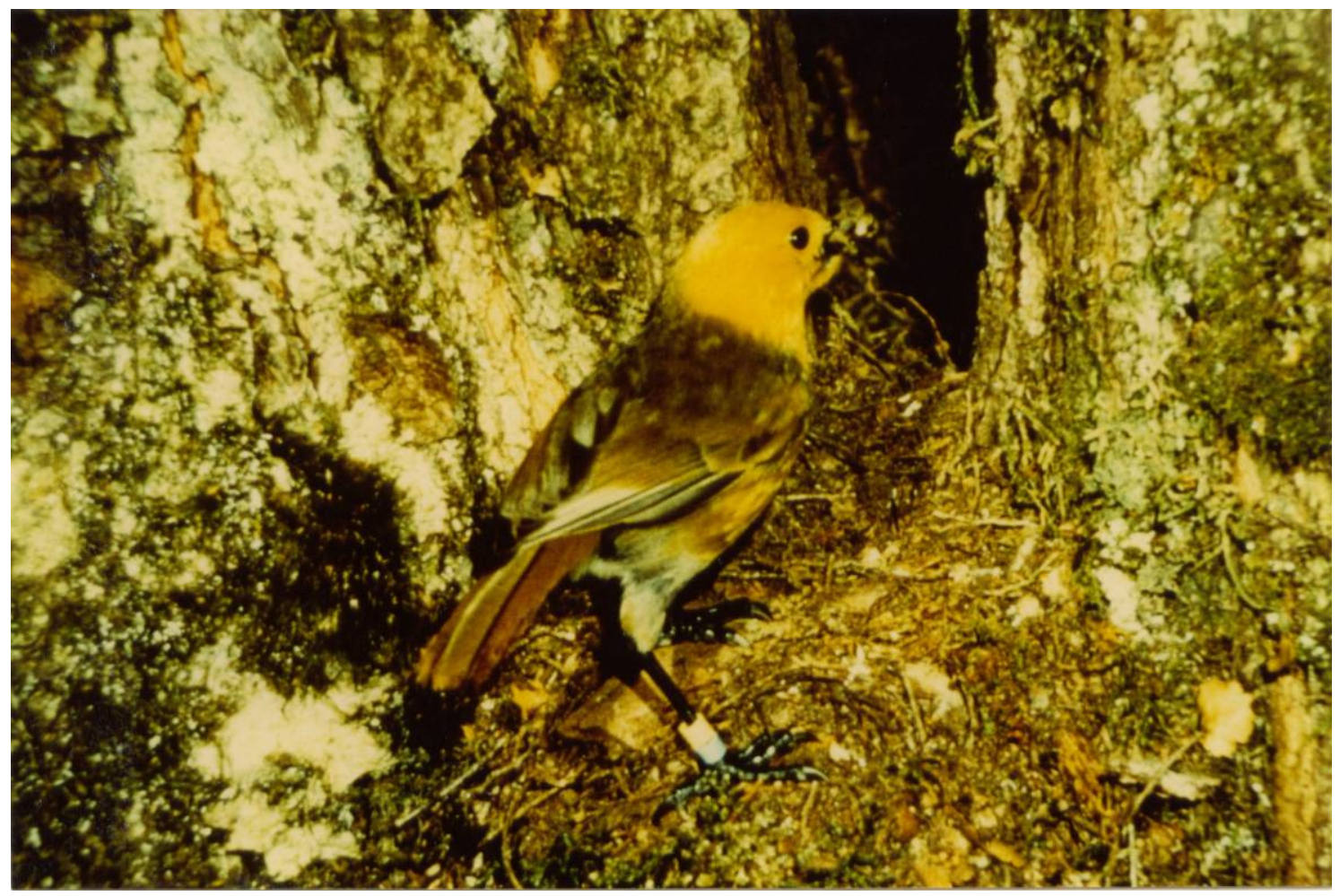




\section{ABSTRACT}

This study aimed to find an explanation for the decline of yellowheads and formulate recommendations for management and further research on the species. There were three main lines of investigation: basic population ecology and behaviour; the effect of introduced predators on breeding; and the habitat relationships of the species. A detailed study of a yellowhead population in the Eglinton Valley in Fiordland National Park was undertaken. Birds were caught and banded and their behaviour, breeding and survival monitored for 4 years. The relationship between yellowhead distribution and vegetation, topography, and fertility were investigated in part of Mt Aspiring National Park during one summer.

Yellowheads suffered high rates of predation from stoats during "plagues" that occurred after heavy beech seeding. Three aspects of yellowhead biology made them vulnerable to mammalian predation: (1) they nested in holes and predators killed not only eggs and nestlings, but also incubating adults; (2) only the females incubated, thus losses to predators had a greater effect on the population than if equal numbers of males and females were killed; and (3) yellowheads nested later than most other forest passerines and were still nesting when stoat numbers reached their summer peak. Though the yellowhead's hole nesting habit made them vulnerable to mammals it restricted nest parasitism and predation by long-tailed cuckoos and hole nesting is likely to have evolved in response to cuckoos.

Yellowheads were found to be tall forest specialists; they occurred more frequently in tall forests than short ones, and preferentially used the largest trees. Their choice of nest sites had no effect on their preference for any forest types. The forests they favoured grew mainly on fertile valley floors at low altitudes.

Yellowhead populations in "good habitats" raised two broods a year and these populations are probably sufficiently productive to withstand stoat plagues occurring once every 5 years, the average frequency of this event. Populations in "poor habitats" raise only one brood and their productivity is probably insufficient to match losses to stoats. Such populations are probably slowly declining, and are very vulnerable to extinction.

A habitat suitability index was devised and forests in the north of the South Island from which yellowheads have disappeared, were compared with those in the south where yellowheads persist. Northern forests were as good for yellowheads as southern ones. 
Thus, the combination of habitat preference and predation cannot account for the recent disappearance of yellowheads from the northern half of the South Island.

The decline in yellowheads was attributed to both predation by introduced mammals and competition with introduced vespulid wasps. Predation may have eliminated yellowheads from podocarp-dominated forests where predator numbers are constantly high, but they survive in some beech forests where predator numbers rise only once every five years. However, even within beech forests only the most productive populations are sufficiently productive to survive predation and these populations are probably susceptible to competition with wasps which eat large numbers of invertebrates.

Yellowheads are likely to be more vulnerable to wasp competition than other forest insectivores because: (1) predation has reduced their productivity more than other birds because they nest in holes; (2) they are specialised in low altitude, tall forest that the wasps also favour; (3) their breeding is later than most other forest birds and their period of juvenile dependence much longer. Yellowheads are still feeding fledgling yellowheads at the time when wasps numbers reach their peak in the autumn, whereas the offspring of other forest birds are independent by this stage. 


\section{TABLE OF CONTENTS}

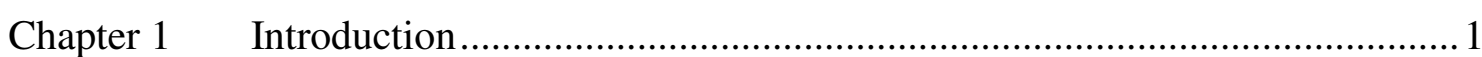

1.1 Taxonomic relationships of the yellowhead .........................................2

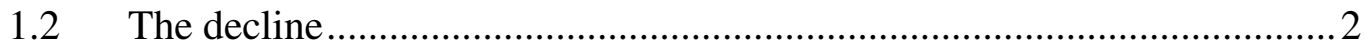

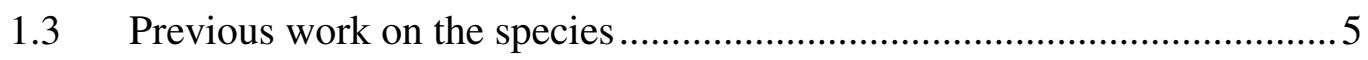

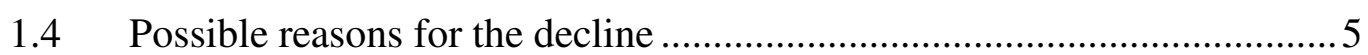

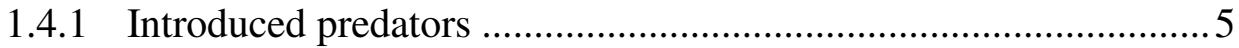

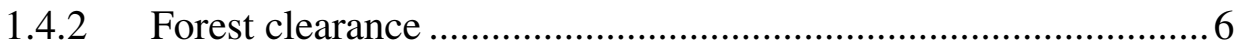

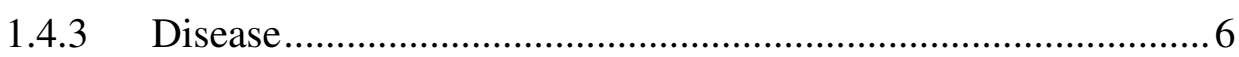

1.4.4 Introduced vespulid wasps................................................. 7

1.4.5 Habitat specialisation..........................................................

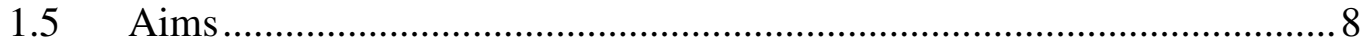

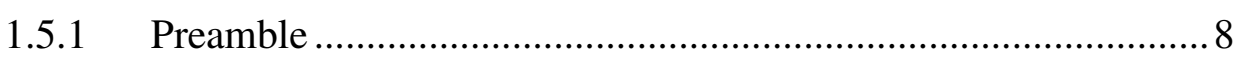

1.5.2 Population ecology and behaviour......................................... 8

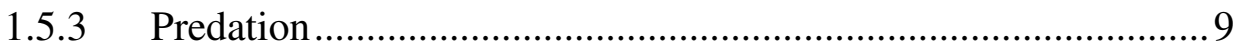

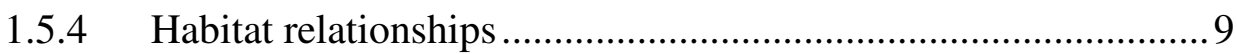

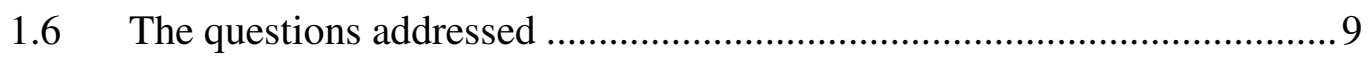

1.6.1 Population ecology and behaviour.......................................... 9

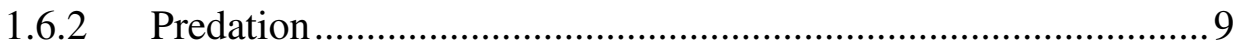

1.6.3 Habitat relationships ........................................................ 10

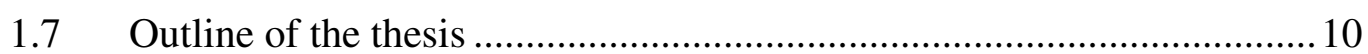

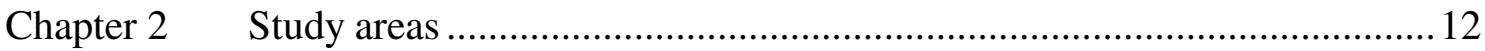

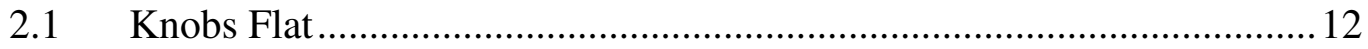

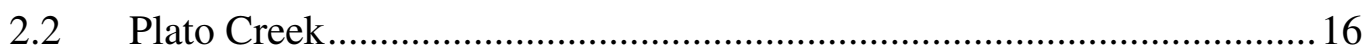

Section One The yellowhead................................................................... 18

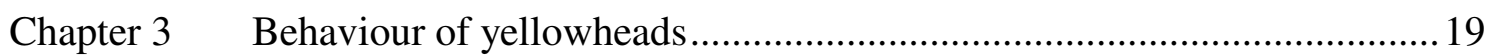

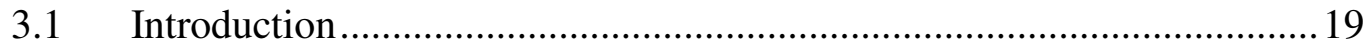

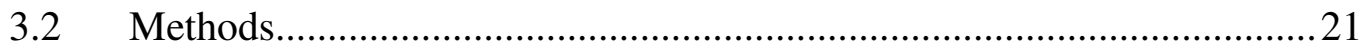

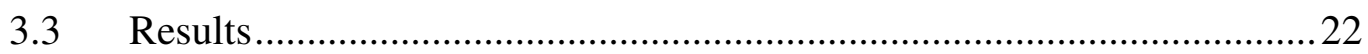

3.3.1 Behavioural differences between males and females ............... 22

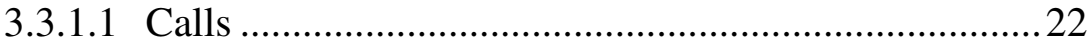

3.3.1.2 Territorial behaviour ........................................ 22

3.3.1.3 Courtship feeding ............................................ 22

3.3.1.4 Nesting ................................................................. 23 
3.3.2 Behavioural differences with age …....................................23

3.3.2.1 Juvenile .......................................................... 23

3.3.2.2 Immature ....................................................... 24

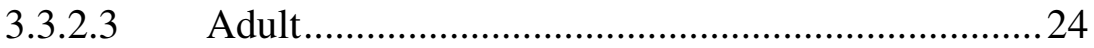

3.3.3 Behaviour during the breeding season...................................25

3.3.3.1 Primary adults.................................................. 25

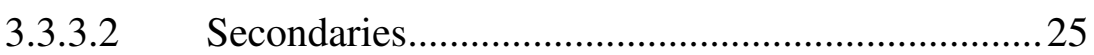

3.3.3.2.1 Adult secondaries ...............................25

3.3.3.2.2 Immature secondaries .........................26

3.3.3.3 Secondary pairs .............................................. 27

3.3.3.4 Territory defence ........................................... 27

3.3.3.5 Territory and mate fidelity.................................... 28

3.3.3.6 Establishing new territories ................................. 28

3.3.4 Behaviour outside the breeding season..................................... 30

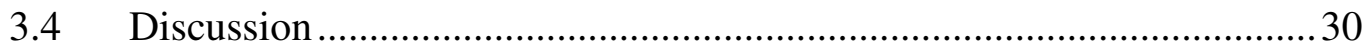

chapter $4 \quad$ Plumage and morphometrics of yellowheads ....................................... 33

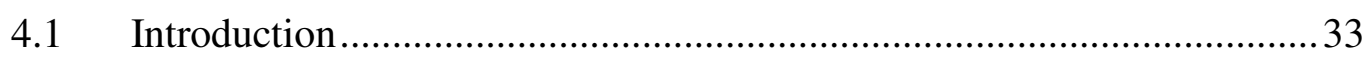

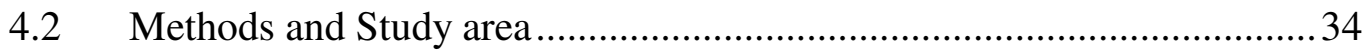

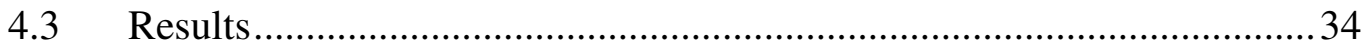

4.3.1 Adult male and female plumages ……................................... 34

4.3.2 Age-related plumages .......................................................... 34

4.3.2.1 Juvenile plumage ................................................ 35

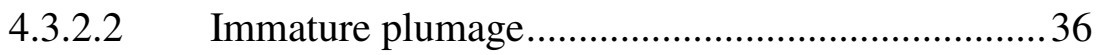

4.3.2.3 Adult plumage ................................................... 36

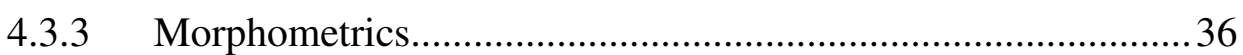

4.3.3.1 Morphometrics of pairs ...................................... 37

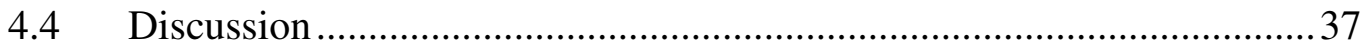

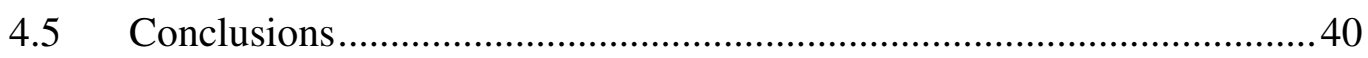

Section 2 The effect of predation on yellowheads............................4

Chapter 5 Productivity and Mortality of yellowheads ........................................... 42

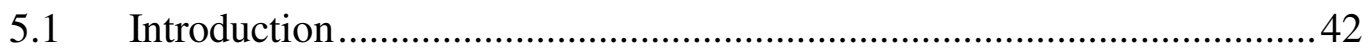

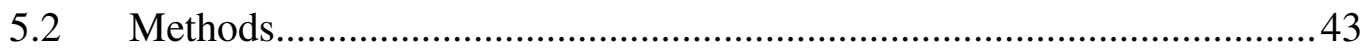

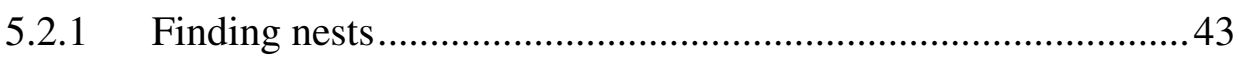

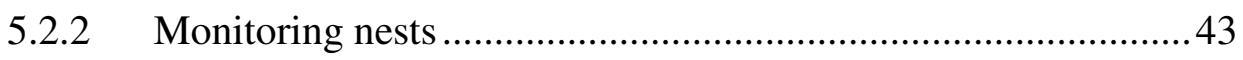

5.2.2.1 Climbing up to nests............................................ 43 
5.2.3 Catching, banding and measuring birds..................................4 44

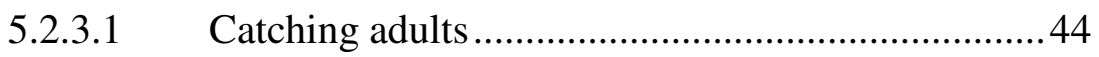

5.2.3.2 Catching fledglings and nestlings......................... 44

5.2.3.3 Banding ........................................................ 45

5.2.3.4 Measurements.................................................. 45

5.2.3.5 Sex and age of birds ........................................ 45

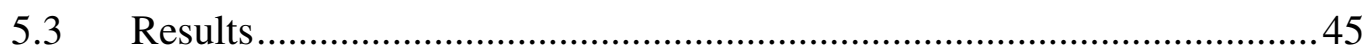

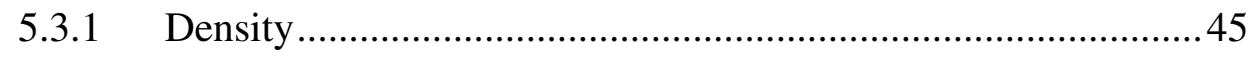

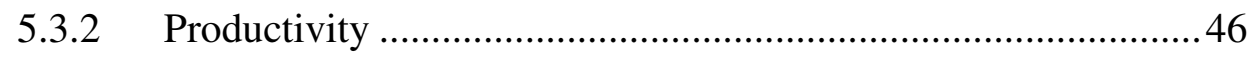

5.3.2.1 Number of clutches ............................................. 46

5.3.2.2 Timing of breeding ............................................. 47

5.3.2.3 Nest building ........................................................ 49

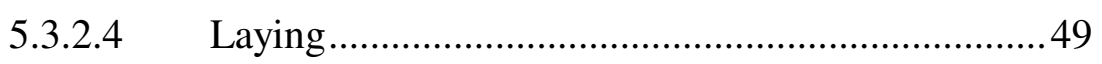

5.3.2.5 Incubation period.................................................... 49

5.3.2.6 Nestling period ....................................................50

5.3.2.7 Clutch size .......................................................... 51

5.3.2.8 Hatching success ..................................................... 52

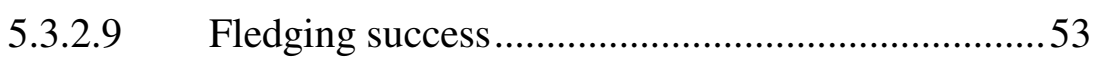

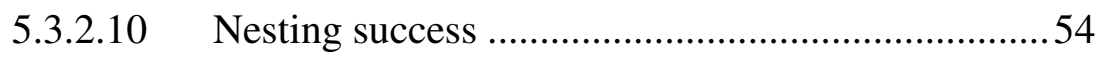

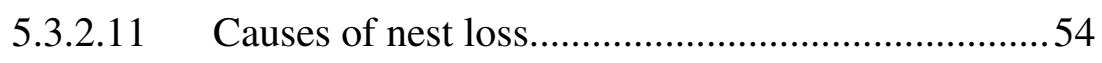

5.3.2.12 Productivity ......................................................56

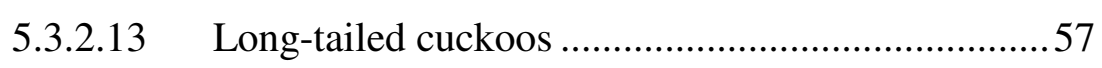

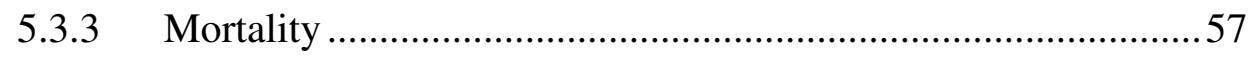

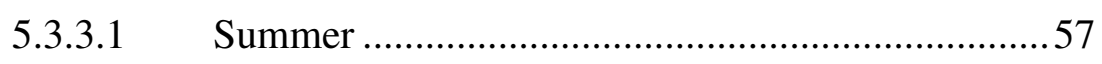

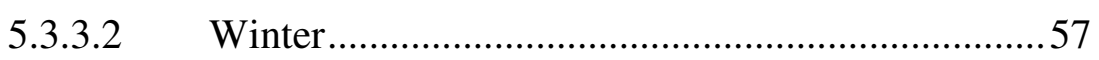

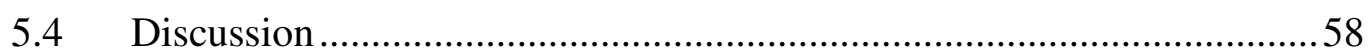

5.4.1 Incubation and nestling periods .........................................58

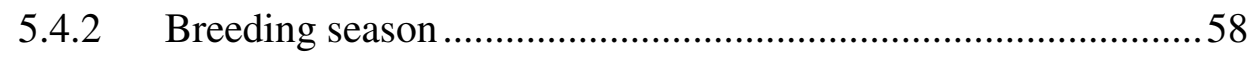

5.4.3 Variation in stoat numbers....................................................6 60

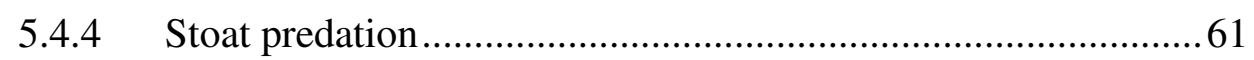

5.4.5 Productivity, mortality and stoat plagues ...............................61

5.4.6 Other predators ................................................................63

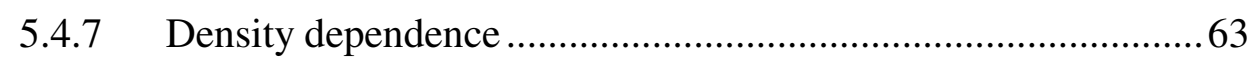

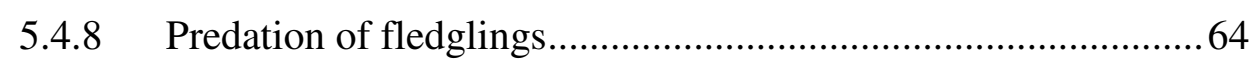

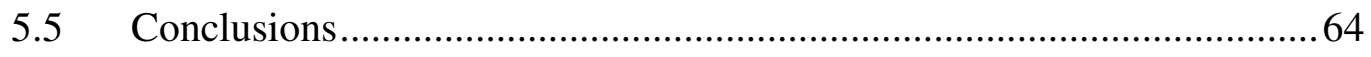

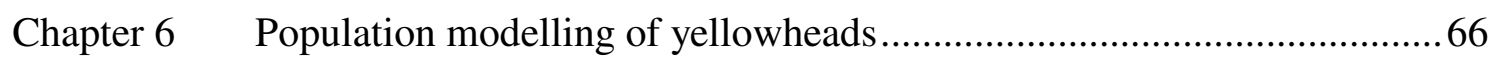

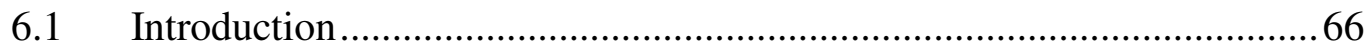




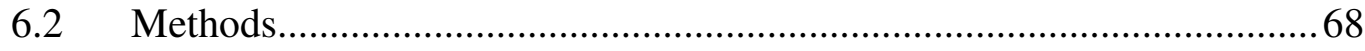

6.2.1 Population parameters .......................................................68

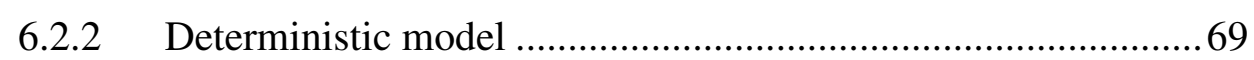

6.2.2.1 Density dependence........................................... 71

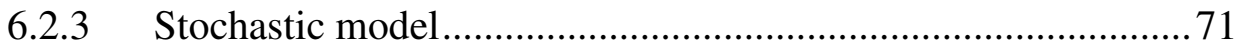

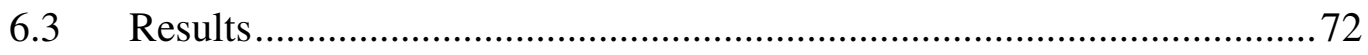

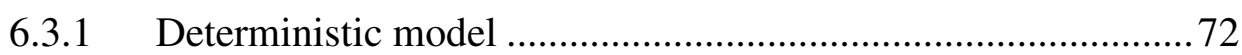

6.3.1.1 Frequency of stoat plagues ..................................73

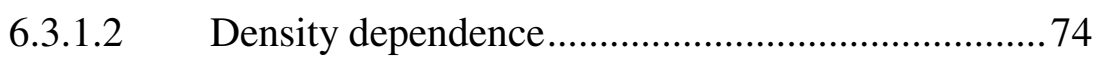

6.3.2 Stochastic model................................................................. 76

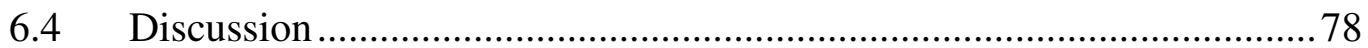

Chapter $7 \quad$ Yellowheads and Long-tailed cuckoos .............................................. 82

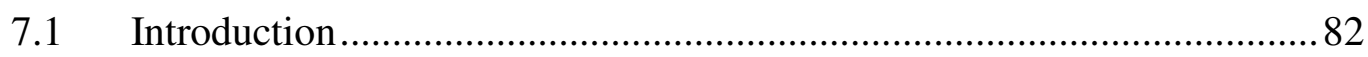

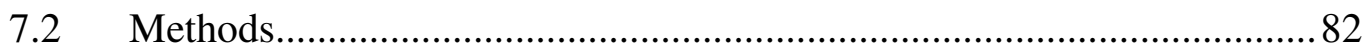

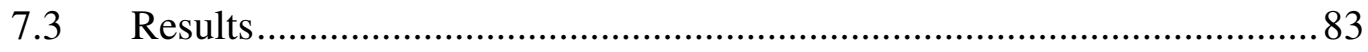

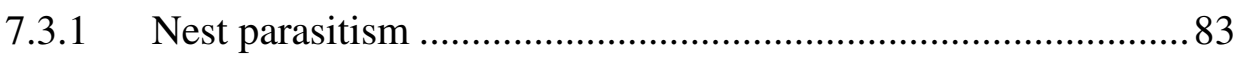

7.3.1.1 Nest sites ...................................................... 83

7.3.1.2 Long-tailed cuckoo eggs.................................... 84

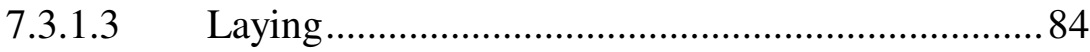

7.3.1.4 Timing of egg laying ......................................... 84

7.3.1.5 Hatching and nestling period................................ 85

7.3.1.6 Fledging to independence..................................... 86

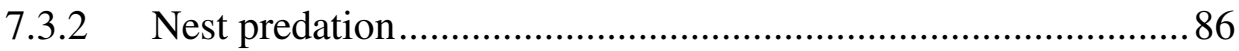

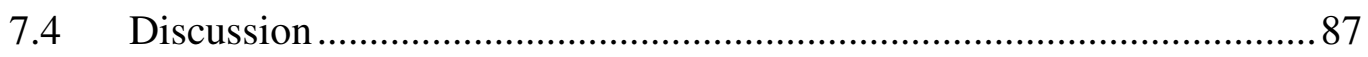

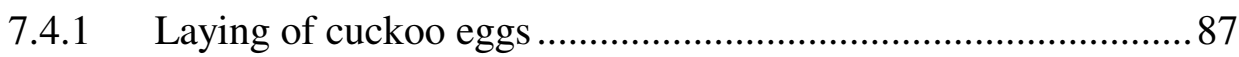

7.4.2 The timing of yellowhead and cuckoo laying ...........................8 87

7.4.3 Fledgling cuckoo behaviour .................................................. 88

7.4.4 The rate of nest parasitism and its effect on yellowhead productivity 88

7.4.5 Does hole nesting protect yellowheads from long-tailed

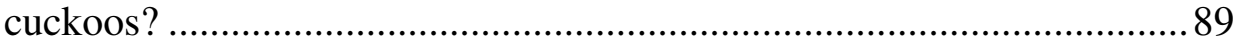

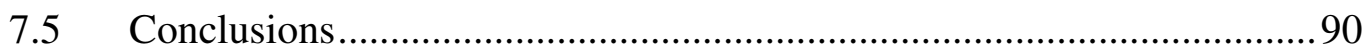

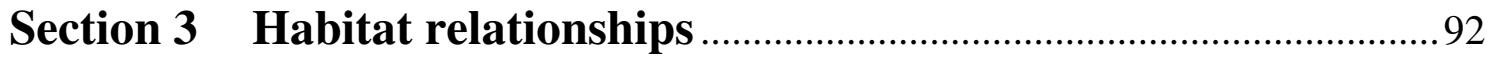

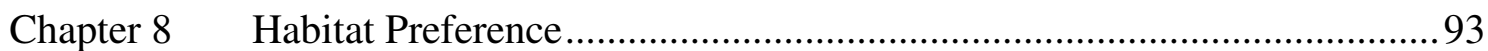

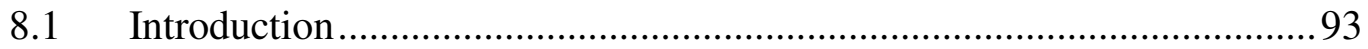




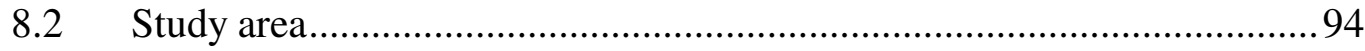

8.2.1 Habitat suitability index study area ........................................94

8.2.2 Sites for comparison ......................................................... 95

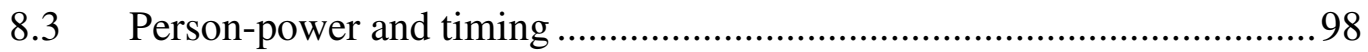

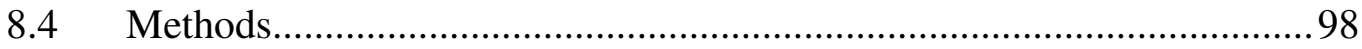

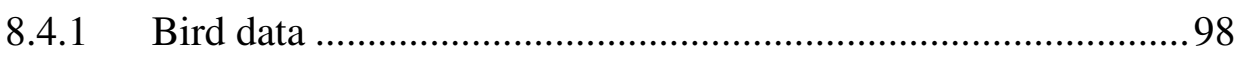

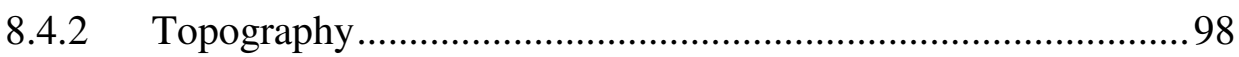

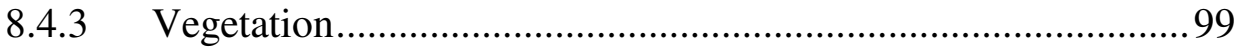

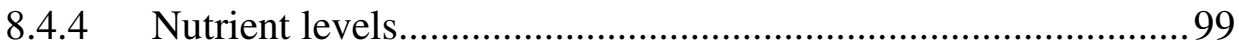

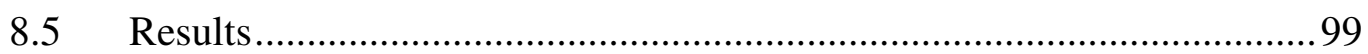

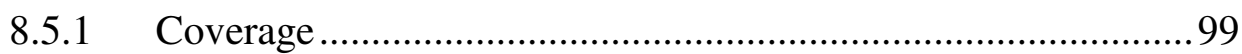

8.5.2 Patterns of yellowhead distribution ......................................... 100

8.5.3 Yellowhead and brown creeper distribution............................. 104

8.5.4 Habitat suitability index...................................................... 105

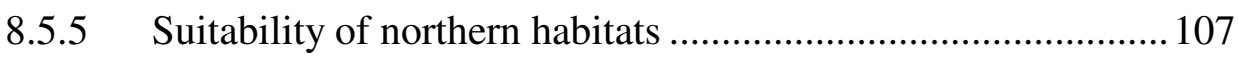

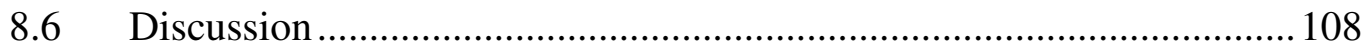

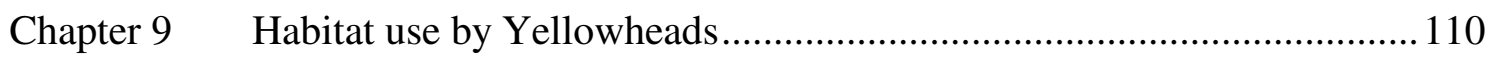

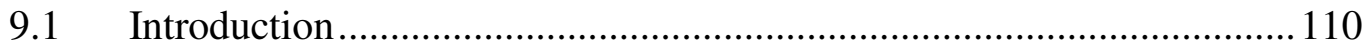

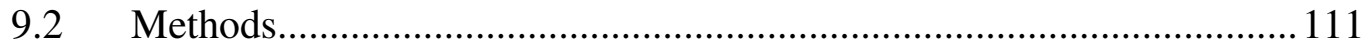

9.2.1 Sampling yellowhead habitat use ....................................... 111

9.2.2 Vegetation sampling .......................................................... 112

9.2.2.1 Variable area plots............................................ 112

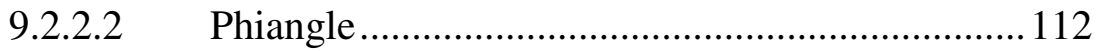

9.2.3 Assessing selective use of trees ........................................... 115

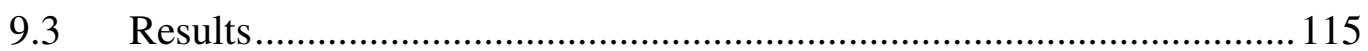

9.3.1 Yellowhead activities ........................................................... 115

9.3.2 Yellowhead use of perch sites ................................................ 117

9.3.3 Yellowheads and tree species ................................................. 119

9.3.4 Yellowheads and tree size ................................................... 120

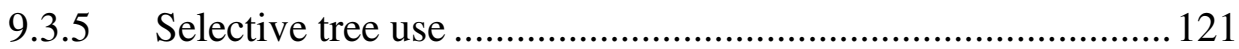

9.3.5.1 Variable area plot measures of tree abundance............................................................ 121

9.3.5.2 Phiangle measures of tree abundance................... 124

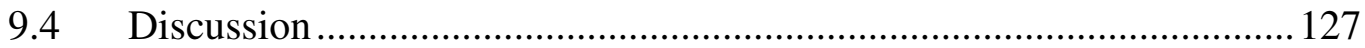

9.4.1 Some problems with the habitat use sampling method ........... 127

9.4.1.1 Pooling and repeated sampling bias .................... 127

9.4.1.2 Visibility bias .................................................... 128 
9.4.1.3 Assessing the magnitude of bias ......................... 128

9.4.2 Foraging behaviour ................................................................. 130

9.4.3 Selective tree use ............................................................. 131

Chapter $10 \quad$ Nest Site Selection by yellowheads ................................................... 133

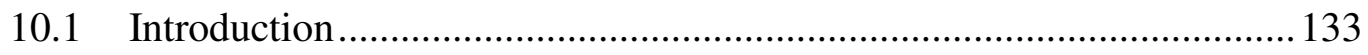

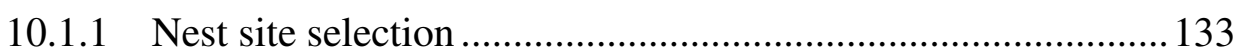

10.1.2 Nest sites and habitat preference .......................................... 133

10.1.3 The evolution of hole nesting in yellowheads ........................ 134

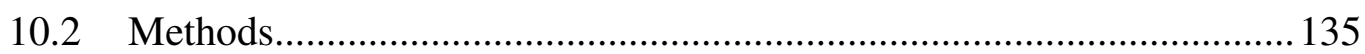

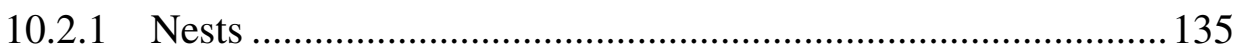

10.2.2 Vegetation measurement ................................................... 136

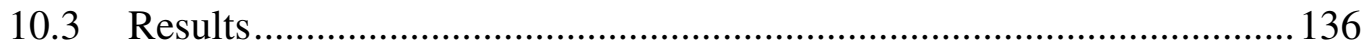

10.3.1 Description of nests ............................................................ 136

10.3.2 Successful and unsuccessful nest sites ................................. 137

10.3.3 Nest site selectivity ............................................................. 138

10.3.3.1 Re-use and knowledge of a nest site ................... 139

10.3.3.2 Re-use of successful nests ................................... 140

10.3.3.3 Inherent preferability of nest sites ....................... 141

10.3.4 Nest site - vegetation relationships ...................................... 142

10.3.4.1 Vegetation near the nest ................................... 142

10.3.4.2 Nest sites and trees .............................................. 143

10.3.4.3 Direction of nest holes....................................... 147

10.3.4.4 Nest hole entrance size ...................................... 148

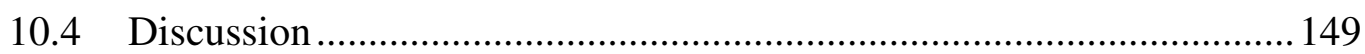

10.4.1 Nest site selectivity ....................................................... 149

10.4.2 Nest sites and habitat preferences......................................... 149

10.4.3 Evolution of hole nesting .................................................... 150

10.4.3.1 Nest site density............................................... 151

10.4.3.2 Competition for nest sites.................................... 153

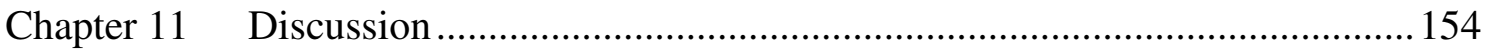

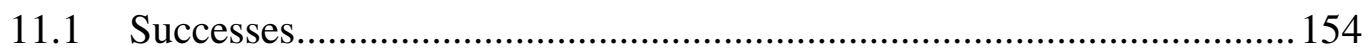

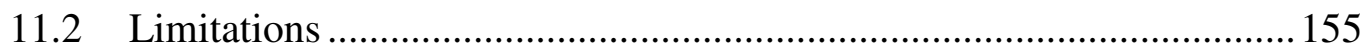

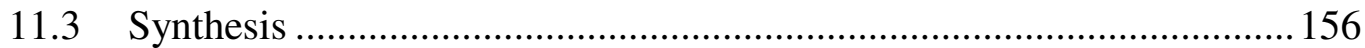

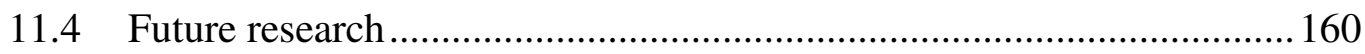

11.4.1 Research for management.................................................. 160

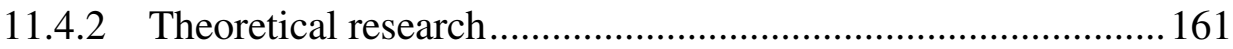


References

Appendix A Basic deterministic population simulation

Appendix B Fortran stochastic population simulation

Appendix C A New Method of measuring vegetation for studies of bird habitat use: Point height intercept analysis at an angle 179

C.1 Introduction. 179

C. 2 The new method 180

C.2.1 Background to the new Method. 180

C.2.2 The new method 181

C.3 References 


\section{LIST OF TABLES}

Table

Page

4.1 Comparison of measurements of male and female yellowheads .36

4.2 The relative sizes of males and females in 12 pairs of yellowhead .37

The probability of a female yellowhead being larger than its mate in randomly selected pairs.

4.4 Test of hypothesis that male and female yellowheads randomly assort 39

4.5 The probability of a female whitehead being larger than its mate in randomly selected pairs

Test of hypothesis that male and female whiteheads randomly assort 40

5.1 Numbers of yellowheads in November at Knobs Flat. .46

5.3 Variation in the timing of breeding between years at Knobs Flat ................48

Clutch sizes of first and subsequent clutches and Mann-Whitney test 51

Variation in clutch size between months and Kruskal-Wallis test ...... .52

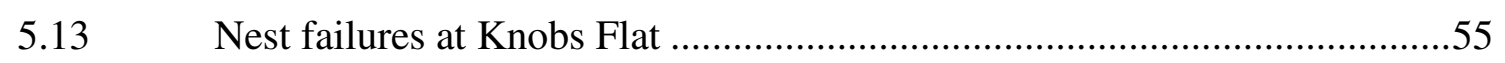

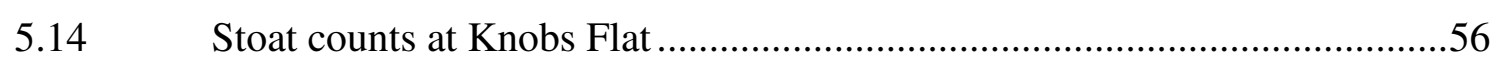

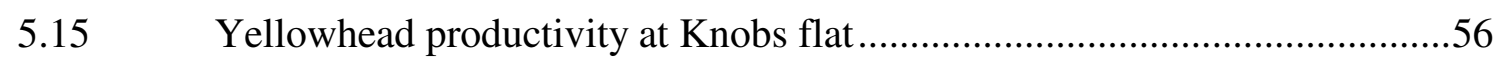

5.16 Population structure and winter mortality rates at Knobs Flat .....................58

5.17 Breeding success of New Zealand forest-dwelling insectivorous passerines

5.18 Survival rates of New Zealand forest-dwelling insectivorous passerines

6.1 Population parameters of yellowheads at Knobs Flat between 1984 and 1989

6.2 Population projection matrices and growth rates of one and two brood yellowhead populations

7.1 Comparison of long-tailed-cuckoo-parasitised and unparasitised yellowhead nests

8.1 Differences between places with and without yellowheads continuous variables. 
8.2 Differences between places with and without yellowheads discontinuous variables

8.3 Differences between places with high and low numbers of yellowheads - continuous variables

8.4 Differences between places with high and low numbers of yellowheads - discontinuous variables

8.5 Contingency table test of association between the distribution of yellowheads and brown creepers

Results of stepwise logistic regression

9.1 The distribution of observations amongst activity classes and tree species 117

9.2 The distribution of observations amongst perch sites and tree species 119

9.3 The distribution of observations amongst species and size classes of tree 122

9.4 The distribution of observations amongst tree species

9.5 The distribution of observations amongst size classes of tree

9.6 The distribution of observations amongst species of tree and goodness of fit test of hypotheses that yellowheads use tree species in proportion to their surface areas as measured using phiangle 124

9.7 The distribution of observations amongst size classes of tree and goodness of fit test of hypotheses that yellowheads use size classes in the proportion to their surface areas as measured using phiangleof fit test of hypotheses that yellowheads use size classes in the proportion to their surface areas as measured using phiangle 125

9.8 The distribution of observations amongst perch sites and goodness of fit test of hypotheses that yellowheads use perch sites in proportion to their surface areas as measured using phiangleness of fit test of hypotheses that yellowheads use perch sites in proportion to their surface areas as measured using phiangle .126

9.9 The distribution of observations amongst height strata and goodness of fit test of hypotheses that yellowheads use height strata in proportion to their surface areas as measured using phiangless of fit test of hypotheses that yellowheads use height strata in proportion to their surface areas as measured using phiangle 126 
9.10 The distribution of observations amongst height strata and goodness of fit test of hypotheses that yellowheads use height strata in proportion to their surface areas as measured using phiangle in re fit test of hypotheses that yellowheads use height strata in proportion to their surface areas as measured using phiangle in red and silver beech trees

9.11 Test for the effect of sampling regime on the distribution of observations amongst activities

9.12 Test for the effect of sampling regime on the distribution of observations amongst tree species and size classes Association between nest success and tree species.

Observed and expected frequency of nests in species by size classes 146

$10.11 \quad$ Nest site direction 


\section{LIST OF FIGURES}

Figure

1.1

2.1

2.2

2.3

2.4

2.5

2.6

3.1

4.1

5.3

5.4

6.1

6.2

6.3

6.4

\section{Page}

The distribution of yellowheads in 1850 and 1990 4

The location of the Knobs Flat and Plato Creek study areas..... .13

An aerial view of the Eglinton Valley at Knobs Flat ....................................14

The Eglinton Valley at Knobs Flat..........................................................14

Silver beech dominated forest in the Knobs Flat study area ........................15

Red beech dominated forest in the Knobs Flat study area ..... 15

The Knobs Flat study area. 17

The locations of yellowhead territories and their occupants at Knobs Flat between 1984 and 1988. .29

A juvenile yellowhead. .35

Timing of nesting 48

Clutch size. .51

Fledgling survival rates used in density dependent yellowhead population simulation. .69

Stoat plague frequency and yellowhead population growth rates .74

Density dependent population simulations for one and two-brood yellowhead populations .75

The effect of infrequent stoat plagues on the sex ratio of a two brood yellowhead population. .76

The relationship between probability of survival, and carrying capacity for two-brood populations suffering stoat plagues every 5 years

Growth curve for a two-brood yellowhead population with a carrying capacity of 30 pairs and suffering stoat plagues every five years .77

The relationship between probability of survival, and carrying capacity for one-brood populations suffering stoat plagues every 5 years .78

A comparison of the entrance hole cross-sectional area of yellowhead nests parasitised by long-tailed cuckoos, with those not parasitised by long-tailed cuckoos .84

The beginning of incubation at cuckoo-parasitised and unparasitised yellowhead nests .85

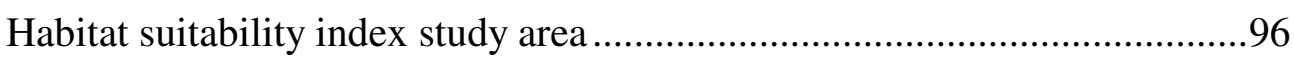

Comparison sites in the northern South Island. .97 
8.3 Survey coverage and distribution of yellowheads within the study

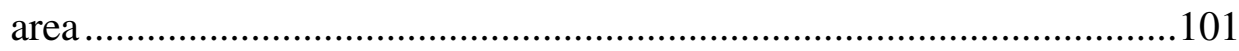

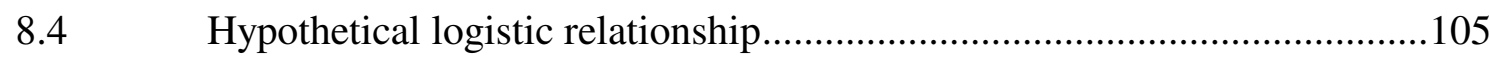

8.5 Habitat suitability of sites in Mt Aspiring National Park, and the

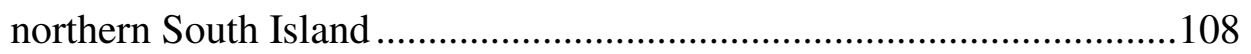

9.1 Variable area vegetation plots in the Knobs Flat study area ......................113

9.2 Phiangle vegetation plots in the Knobs Flat study area.............................. 114

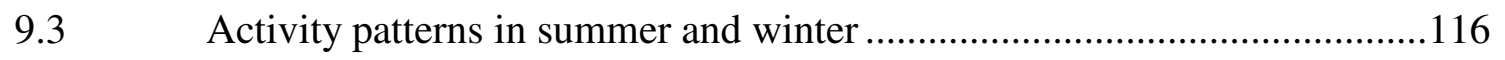

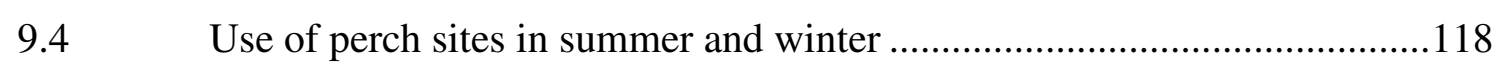

9.5 Use of different tree species in summer and winter ...................................120

$9.6 \quad$ Use of different tree sizes in summer and winter....................................... 121

10.1 Frequency distribution of nest hole entrance size ....................................... 148

C.1 The arrangement of phiangle sightings with respect to the grid at

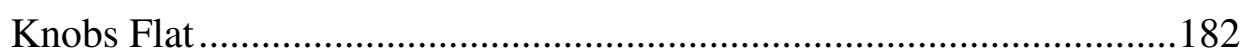




\section{CHAPTER 1}

\section{INTRODUCTION}

The yellowhead (Mohoua ochrocephala (Gmelin 1789)) is an endemic forest-dwelling passerine found in the South Island of New Zealand. Last century it was found throughout the forests of the South and Stewart Islands, but during the last 30 years it has contracted in range from the north southwards and is now virtually confined to large forest blocks in the south of the South Island (Gaze 1985).

Yellowheads are about the size of house sparrows (Passer domesticus). Their diet consists mainly of insects and spiders that they pick off the surface of leaves and bark, though they occasionally scratch amongst loose bark or accumulations of litter in crooks or on the ground. They occasionally take small fruit. They spend most of their time foraging high in the forest canopy. For the last 50 years they have been found only in beech (Nothofagus) forests of the South Island, where they appear to be in highest densities in tall forest on valley floors.

Following European colonisation of New Zealand, some New Zealand birds, such as huia (Heteralocha acutirostris), saddleback (Philesturnus carunculatus) and kakapo (Strigops habroptilus) declined rapidly to extinction or near extinction. Endangered species such as these have been the focus of recent conservation work, but there has been a growing realisation that, for better or worse, their futures are already decided and that attention should now shift to less immediately endangered species. Some (e.g., Williams 1986) have even suggested that some "hopeless case" endangered species should be abandoned in favour of conservation management of threatened species for which there is a better chance of success.

The yellowhead is one of the top priorities amongst forest-dwelling threatened species for conservation work (Crawley 1981). Its range has shrunk considerably and is still apparently contracting, but it still seems possible that populations of the species can be maintained on the South Island mainland.

This thesis reports on my attempt to find reasons for the yellowhead's decline and to identify action which could halt it. It is a case study of a threatened New Zealand forest bird. 


\section{$1.1 \quad$ TAXONOMIC RELATIONSHIPS OF THE YELLOWHEAD}

The yellowhead and brown creeper (Finschia novaeseelandiae) of the South and Stewart Islands, and the whitehead (Mohoua albicilla) of the North Island, together form a closely related group of forest dwelling passerines with rather obscure relationships to other birds.

The yellowhead and whitehead were originally placed in different families, but their close relationship was soon recognised and they have been considered congeners since 1845 (Fleming 1982). Similarly they were originally placed in a different family to the brown creeper, but the three species are now widely recognised as being close relatives and the most recent work suggests they belong in the same genus (Sibley and Ahlquist 1987).

The relationships of these three species with other southern hemisphere birds have until recently been unclear. Oliver (1955) placed them with the fernbird (Bowdleria punctata) in the Sylviidae (warblers), as the only members of the family in New Zealand. Kinsky (1970) placed them with the Gerygone warblers in Malurinae (Australian warblers), a sub-family of the hold-all family Muscicapidae. Keast (1977) believed they were derived from "... an early generalized, forest-dwelling protopachycephaline stock" and their closest relatives were the pachycephaline warblers of Australia. Fleming (1982) gave little credence to Keast's ideas, but recent DNA hybridization work by Sibley and Ahlquist (1987) supports Keast's hypothesis. The yellowhead, whitehead and brown creeper probably belong to a group of Australasian passerines not closely related to the predominantly northern hemisphere sylviids or muscicapids with which they had previously been placed (Sibley and Ahlquist 1985, 1987).

Fleming (1982) believed that yellowheads, whiteheads and brown creepers differentiated during the Pleistocene. Whiteheads became forest and scrub dwellers in the North Island, while yellowheads and brown creepers shared these habitats in the South; brown creepers became specialised scrub and low forest birds, while yellowheads specialised in tall forest. 
At the beginning of European settlement of New Zealand yellowheads were found throughout the forests of the South and Stewart Islands (Gaze 1985). They were originally found in the podocarp-dominated forests of central Westland and Stewart Island, but between 1850 and 1900 they disappeared from these forests. They had gone from central Westland by about 1900 (Pascoe 1957), and though they were certainly present on Stewart Island (Gaze 1985) they disappeared so early that there has been confusion as to whether or not they were ever there (Williams 1962). On the east coast of the South Island their disappearance coincided with the clearance of forest, but in beech forests throughout the rest of the South Island they initially remained common and have only slowly declined (Gaze 1985). In the last 20 years they have disappeared from large parts of their former range; this may represent the end of a slow steady decline or a recently accelerated decline.

Except for a remnant population of about 20 birds in the Marlborough Sounds, there are no known yellowhead populations in the South Island north of the Hurunui River, and none have been reported from Nelson or north Westland for about 5 years. There are small, scattered populations in Arthur's Pass National Park and Lake Sumner Forest Park, and a few birds in south Westland. In Fiordland and Mt Aspiring National Parks yellowheads are still widespread and even common in some valleys east of the main divide. There are a few populations in isolated forest blocks in Southland. Figure 1.1 compares the present distribution of yellowheads with their distribution last century.

Though yellowhead populations may have been slowly declining since the turn of the century, until the mid 1970's the yellowhead was thought to be common and probably secure in all the large forest blocks of the South Island (e.g. Falla et al. 1979). With publication of the results of a 7 year study of bird distribution in 1978 (Bull et al. 1978) ornithologists realised that it had become quite rare. In 1981 Crawley (1981) ranked work on the "distribution, status and habitat requirements" of yellowheads as a high priority for future work by the New Zealand Wildlife Service, and in 1982 the Ornithological Society of New Zealand began a distribution survey of yellowheads (Gaze 1982). The publication of the results of this survey (Gaze 1985) suggested that the yellowhead should be regarded as "threatened". 


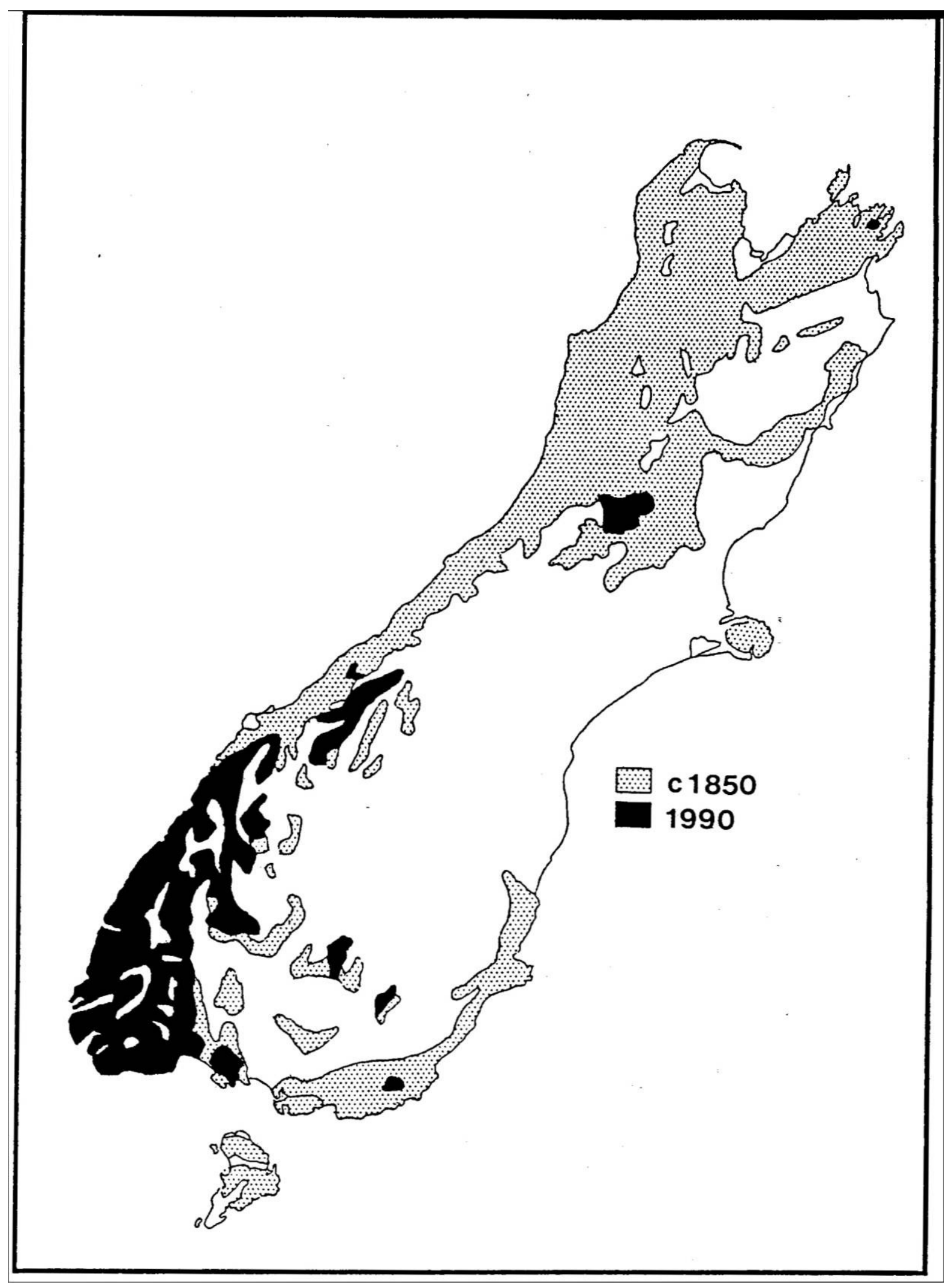

Figure 1.1: $\quad$ The distribution of yellowheads in 1850 and 1990. 


\section{PREVIOUS WORK ON THE SPECIES}

Late last century and early this century a series of descriptive, taxonomic and anecdotal papers about yellowheads were published, mostly in the Transactions and Proceedings of the New Zealand Institute (see Oliver 1968). Through these papers the nomenclature of the species stabilised, and its measurements and some of its habits were recorded.

The first detailed study of yellowhead biology was by Guthrie-Smith (1936), who worked on the Pikikiruna Range near Nelson. He studied the habits of yellowheads during their breeding season and recorded nest sites, clutch sizes, incubation periods and some aspects of their behaviour. In the late 1950's Soper (1960, 1963, 1976) studied similar aspects of breeding yellowheads in the Eglinton Valley in Fiordland.

In the early 1980's, awareness that the yellowhead was becoming rare, and conflict between conservationists and forest managers lead to intensified interest in the species.

In 1981 and 1982 Buckingham (1982) studied the distribution of yellowheads in the Catlins area and made notes on their habitat preferences. In 1983 and 1984 Read (1984, 1987, 1988a, 1988b, Read and O'Donnell 1987) studied patterns of habitat preference and use by yellowheads in the Hawdon Valley in Arthur's Pass National Park. Between 1982 and 1985 Gaze (1982, 1985) collected information on the distribution of the species.

Numerous accounts of yellowhead distribution have appeared in reports of regional bird surveys carried out by the New Zealand Wildlife Service. (e.g. Coker and Imboden 1979, Coker 1980, Elliott and Ogle 1985).

\subsection{POSSIBLE REASONS FOR THE DECLINE}

Five reasons have been suggested for the decline of yellowheads.

\subsubsection{Introduced predators}

Introduced rats (Rattus spp.), mustelids (Mustela spp.) and cats (Felis catus) have been widely blamed for the declines of many native birds (see King 1984). Gaze (1985) suggested that yellowheads might be particularly vulnerable to predation while on the nest and Read (1984) noted that fledgling yellowheads often leave the nest and fall to the ground where they might be vulnerable to cats and mustelids. 
The early disappearance of yellowheads from the podocarp-dominated forests of Stewart Island and central Westland is coincident with the introduction of cats, ship rats (Rattus rattus) and stoats (Mustela erminea). The question arises - why should yellowheads disappear from podocarp forests but not beech forests? Probably because predators reach higher densities in podocarp forests which have a greater variety of fruiting trees. Ship rats are more abundant in diverse podocarp forests than in pure beech forests (Daniel 1976, King 1983). I can find no published comparisons of stoat densities in beech and podocarp forests, but in Nelson Lakes National Park, more stoats have been caught in mixed podocarp-beech forest at low altitude, than have been caught in pure beech forest at higher altitudes (Rowley Taylor pers. comm.).

The yellowhead's hole nesting habits may have a bearing on its susceptibility to predation. The yellowhead is much more rare than either the whitehead or brown creeper, and it is also the only one that nests in holes; the other two have open nests. Soper (1976) suggested that hole nesting was a recently acquired adaptation to reduce nest parasitism and predation by long-tailed cuckoos (Eudynamys taitensis). Alerstam and Högstedt (1981) said that although hole nesting may provide significant protection from avian predators that find their prey by sight, it might make nests more vulnerable to predation by mammals which find their prey by smell.

The recent disappearance of yellowheads from beech forests in the northern South Island, is not easily explained by predation because there is no evidence that predators are any more numerous in beech forests in the north than the south.

\subsubsection{Forest clearance}

Forest clearance probably accounts for the disappearance of yellowheads from most of the east coast of the South Island where there is no longer any forest, but it does not account for their disappearance from large areas of intact forest in Nelson and North Westland.

\subsubsection{Disease}

Disease has long been suspected as a contributory cause of native bird declines in New Zealand (Williams 1973). To account for the decline of yellowheads disease would have to be more prevalent in the north than the south and there is no evidence that this is so. There is almost no information on diseases in wild native birds. 


\subsubsection{Introduced vespulid wasps}

The spread of German wasps (Vespula germanica) into the northern South Island is coincident with the virtual extinction of yellowheads there. Wasps probably compete with yellowheads for food as recent work has shown that they take large numbers of caterpillars from beech trees during the summer (Richard Harris pers. comm.) as do yellowheads (pers. obs.). German wasps established in the North Island near Hamilton in about 1945 and became established in the South Island about 10 years later. They quickly became common in some forested areas (Fordham 1961, 1962), and are now very abundant in the honeydew beech forests in the northern South Island (Sandlant and Moller 1989), but are virtually absent from beech forests in the south where there is no honeydew. Yellowheads in contrast, were widely reported in the northern South Island up until the late 1950s but their range seems to have steadily contracted since then. They are now absent from honeydew forests, which occur to the north of Christchurch in the east, and Greymouth in the west.

Though German wasps may have all but eliminated yellowheads from the northern South Island by the beginning of the 1980's, the arrival of common wasps (Vespula vulgaris) at that time may have sealed their fate, as it caused a net increase in the density of wasps in honeydew beech forests (Sandlant and Moller 1989).

\subsubsection{Habitat specialisation}

Buckingham (1982), Elliott and Ogle (1985) and Read (1984) have all shown that even in areas where yellowheads are common, they are not found in uniform densities, nor are they found throughout the forest. They appear to have quite specific habitat requirements. Furthermore, Read (1984) found that even within favoured forest types yellowheads spent a high proportion of their time feeding. This perhaps implies that less favoured forests may have insufficient food to support yellowheads.

Yellowheads, like most other native forest birds, have probably declined in abundance throughout their range since the arrival of mammalian predators Because of the yellowhead's specific habitat requirements, many forests probably never supported high densities of yellowheads, and it is possible that the places from which yellowheads have disappeared are simply those places that supported low density populations, and the places where they survive are the places that supported high density ones. 
AIMS

\subsubsection{Preamble}

This study aims to make a contribution to our understanding of the reasons for the decline of the yellowhead in the hope that this might lead to management to prevent further decline.

From the preceding section it is apparent that there are more likely explanations for the decline of yellowheads than can be investigated in one $\mathrm{PhD}$ study.

When I started this study I suspected that mammalian predation of nests, and wasp competition were the most likely causes of yellowhead decline, but unfortunately study of both was not possible given available time and finance.

Wasps are implicated in the demise of yellowheads because they occur in highest numbers in areas from which yellowheads disappeared a few years after the wasps arrived. This, of course, could be coincidence, and yellowheads may have disappeared from the northern South Island simply because yellowheads have become less common everywhere, and the forests in the north were never as good for yellowheads as the forests in the south. By comparing northern and southern yellowhead habitats I hoped not only to document the habitat requirements of yellowheads, but to further implicate, or exonerate wasps in their demise.

I therefore chose three main lines of research: basic population ecology and behaviour, the effect of predation, and habitat relationships.

\subsubsection{Population ecology and behaviour}

What are the demographic and social characteristics of the yellowhead?

No detailed studies of yellowhead population ecology and behaviour have previously been undertaken. A necessary precursor to understanding why yellowheads have declined is an understanding of their basic biology. Furthermore, such an understanding might reveal previously unsuspected reasons for their decline. I studied the population ecology and behaviour of about 12 pairs of yellowheads living in 30 ha of forest over a period of 4 years. 


\subsubsection{Predation}

Is there evidence that yellowheads have declined throughout their range?

Yellowheads have obviously declined in those areas where they are now extinct, so the question becomes: has the density of surviving populations declined? There are no accurate estimates of historical yellowhead densities, so rather than attempt to compare historical and recent yellowhead densities, I examined the dynamics of existing populations for evidence of decline.

The usual cause of reductions in endemic bird populations in New Zealand is predation by recently introduced predators. Since yellowheads forage high in trees, they are not vulnerable to these predators while feeding. However, they will be more vulnerable in their hole nests, and fledgling yellowheads that spend time on the ground may also be vulnerable (Read 1987).

\subsubsection{Habitat relationships}

Did forests in the northern South Island support lower densities of yellowheads than forests in the south?

It is not possible to compare yellowhead population densities in the past, but if one assumes that population density is related to habitat quality (Berry 1986, Brennan et al. 1986), one can develop measures of habitat quality and compare the quality of yellowhead habitat in the south with that in the north.

\subsection{THE QUESTIONS ADDRESSED}

\subsubsection{Population ecology and behaviour}

1. How much does yellowhead population density vary between years and seasons?

2. What sort of social organisation do yellowheads have and to what extent do they co-operatively breed?

3. What are the main features of their breeding biology?

4. What are the main features of their demography?

\subsubsection{Predation}

5. Does predation by introduced predators during the breeding season reduce yellowhead populations? 
6. Does hole nesting contribute to the yellowhead's susceptibility to predation?

7. Does hole nesting protect yellowheads from nest predation and parasitism by long-tailed cuckoos?

8. Is there evidence that hole nesting is a recently acquired trait?

9. Does hole nesting in yellowheads match the normal explanations for the evolution of hole nesting?

\subsubsection{Habitat relationships}

10. What are the features of good yellowhead habitat?

11. Are forests in the northern South Island worse for yellowheads than those in the south?

12. Do yellowhead patterns of habitat preference result from selective use of some types of tree for foraging?

13. Do yellowhead patterns of habitat preference result from selective use of some types of tree for nesting?

\section{7}

\section{OUTLINE OF THE THESIS}

Most of this thesis is presented as a series of stand-alone chapters, which will later be published separately. To avoid repetition I describe the main study areas separately in the next chapter (Chapter 2). The rest of the thesis is divided into 3 sections and a concluding chapter.

Section One includes two descriptive chapters, one on yellowhead behaviour and social organisation and one on morphometrics and plumage. These two chapters include information on the basic biology of yellowheads that is not covered in the following two sections.

In Section Two I present an investigation of the question - "does predation by mammalian predators during the breeding season reduce yellowhead populations?" Chapter 5 is an account of the productivity and mortality of yellowheads, with special attention to the effect of mammalian predation. In Chapter 6 I use modelling techniques to assess the effect of mammalian predation on the long-term survival prospects of yellowhead populations. In Chapter 7 I assess the relationship between long-tailed cuckoos and hole nesting in yellowheads.

Section Three addresses the question - "are areas that still support yellowhead populations better quality habitat than areas in which yellowheads have become rare or 
extinct?" In Chapter 8 I develop a "habitat suitability index" (Verner et al. 1986) and compare some forests in the southern South Island, where there are still good yellowhead populations, with some forests in the northern South Island, where yellowheads are rare or have disappeared. In Chapter 9 I discuss the relationship between yellowhead habitat preferences and the use yellowheads make of various tree types and parts of trees. In Chapter $10 \mathrm{I}$ investigate the relationship between yellowhead habitat preference and nesting sites.

Chapter 11 is a synthesis of all my findings. 


\section{CHAPTER 2}

\section{STUDY AREAS}

Two main study areas, Knobs Flat and Plato Creek were used in several parts of this study (Figure 2.1). One other study area in Mt Aspiring National Park was used only for a habitat selection study reported on in Chapter 8. It is described in that chapter.

\section{1}

KNOBS FLAT

Knobs Flat $\left(168^{\circ} 01^{\prime} \mathrm{E}, 44^{\circ} 58^{\prime} \mathrm{S}\right)$ is in the Eglinton Valley in Fiordland National Park, $67 \mathrm{~km}$ north of Te Anau on highway 94 between Te Anau and Milford Sound.

The Eglinton Valley runs approximately NNE - SSW. It is a glaciated valley with steep sides and a flat floor which is 0.5 to $1 \mathrm{~km}$ wide. The valley floor has large open grassy areas near the river, but otherwise the valley is forested up to the tree line at about $1000 \mathrm{~m}$ (Figures 2.2 and 2.3).

My study area comprised approximately 33 ha of forest on alluvium on the valley floor at an altitude of $380 \mathrm{~m}$ above sea level. The forest is dominated by red and silver beech (Nothofagus fusca and N. menziesii) with the forest composition ranging from pure stands of silver beech, generally of low stature (approximately $20 \mathrm{~m}$ tall) (Figure 2.4) to tall stands of red beech (up to approximately $40 \mathrm{~m}$ tall) with an understorey of silver beech (Figure 2.5). Under the canopy the forest is generally open, with few understorey plants. The most common understorey plants are mountain toatoa (Phyllocladus alpinus) and broadleaf (Griselinia littoralis). The felling of some tall red beech trees from parts of the study area during the 1930's has led to vigorous regeneration of small diameter silver beech trees in some areas.

Mean monthly temperatures at Knobs Flat range from $0-8^{\circ} \mathrm{C}$ and the rainfall is approximately $2300 \mathrm{~mm}$ per year. 


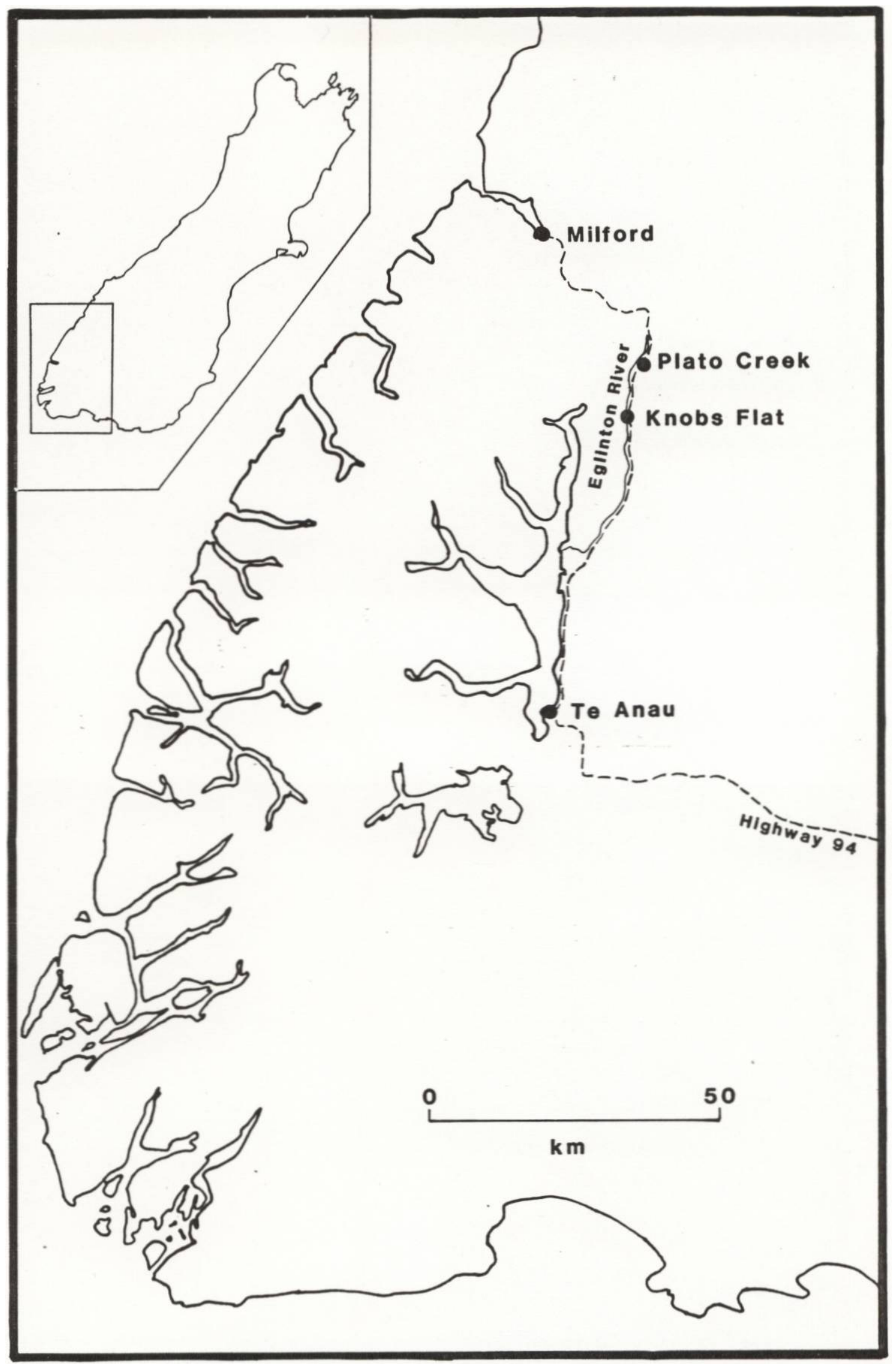

Figure 2.1: $\quad$ The location of the Knobs Flat and Plato Creek study areas. 


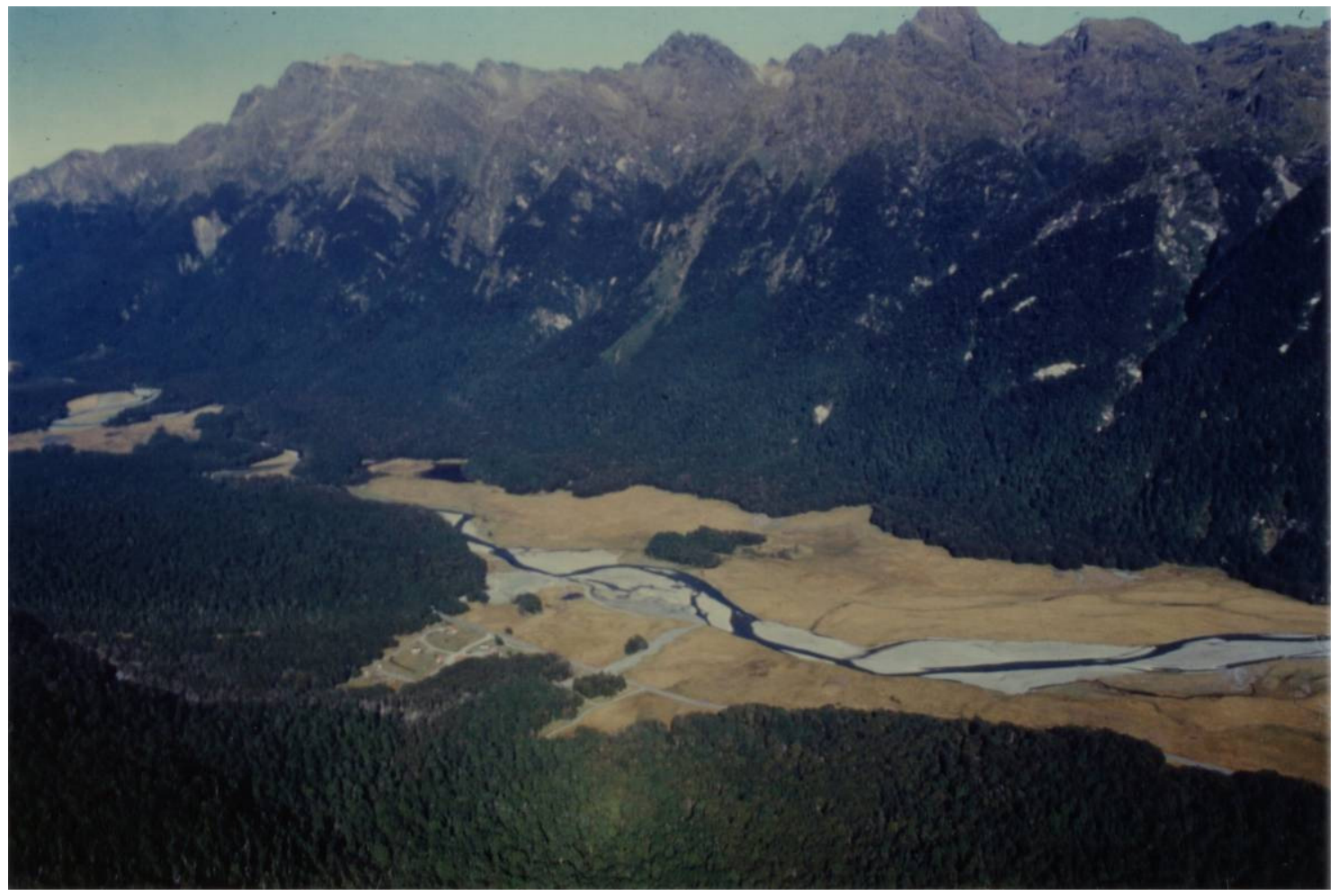

Figure 2.2: $\quad$ An aerial view of the Eglinton Valley at Knobs Flat.

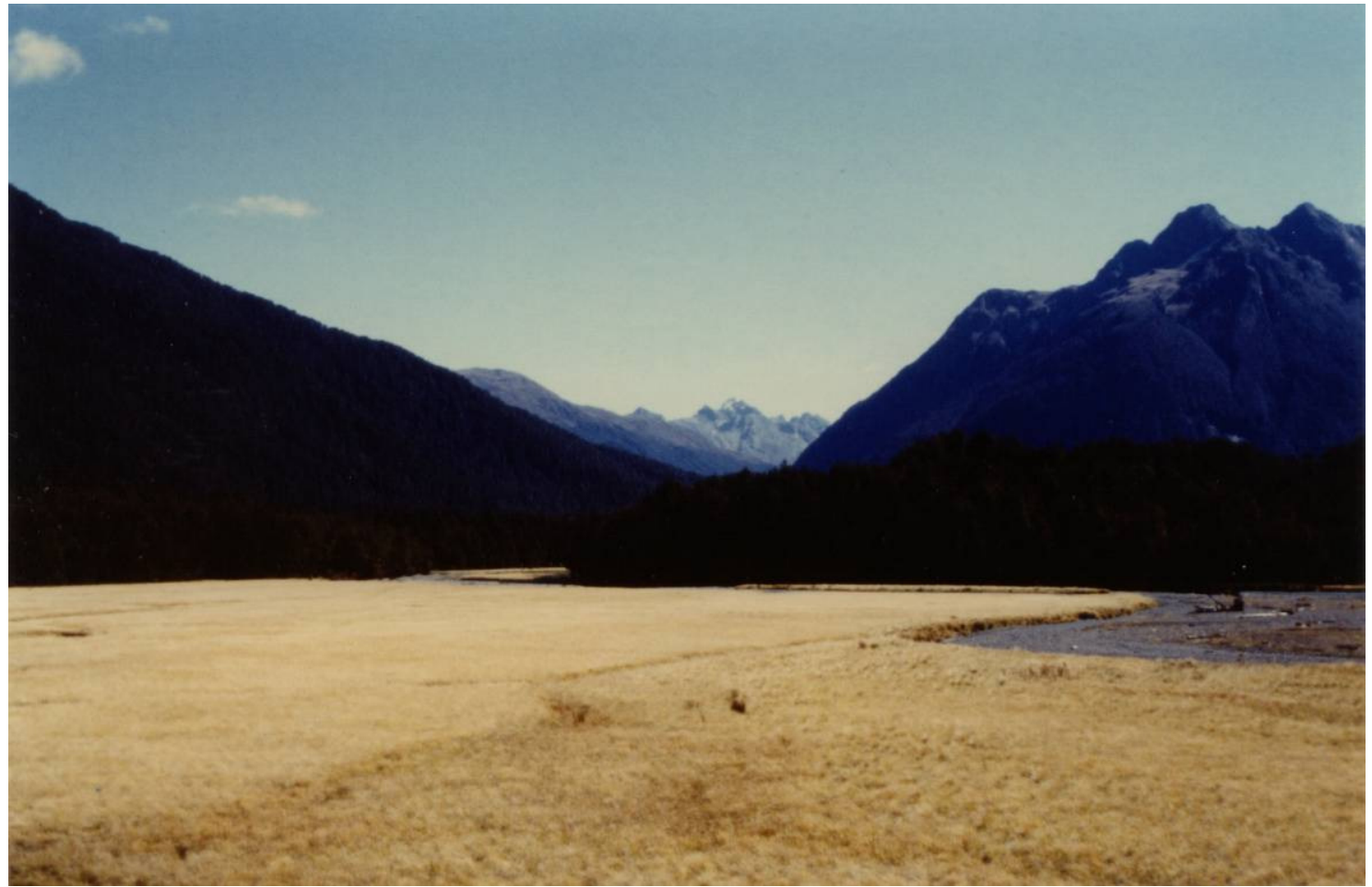

Figure 2.3: $\quad$ The Eglinton Valley at Knobs Flat. 


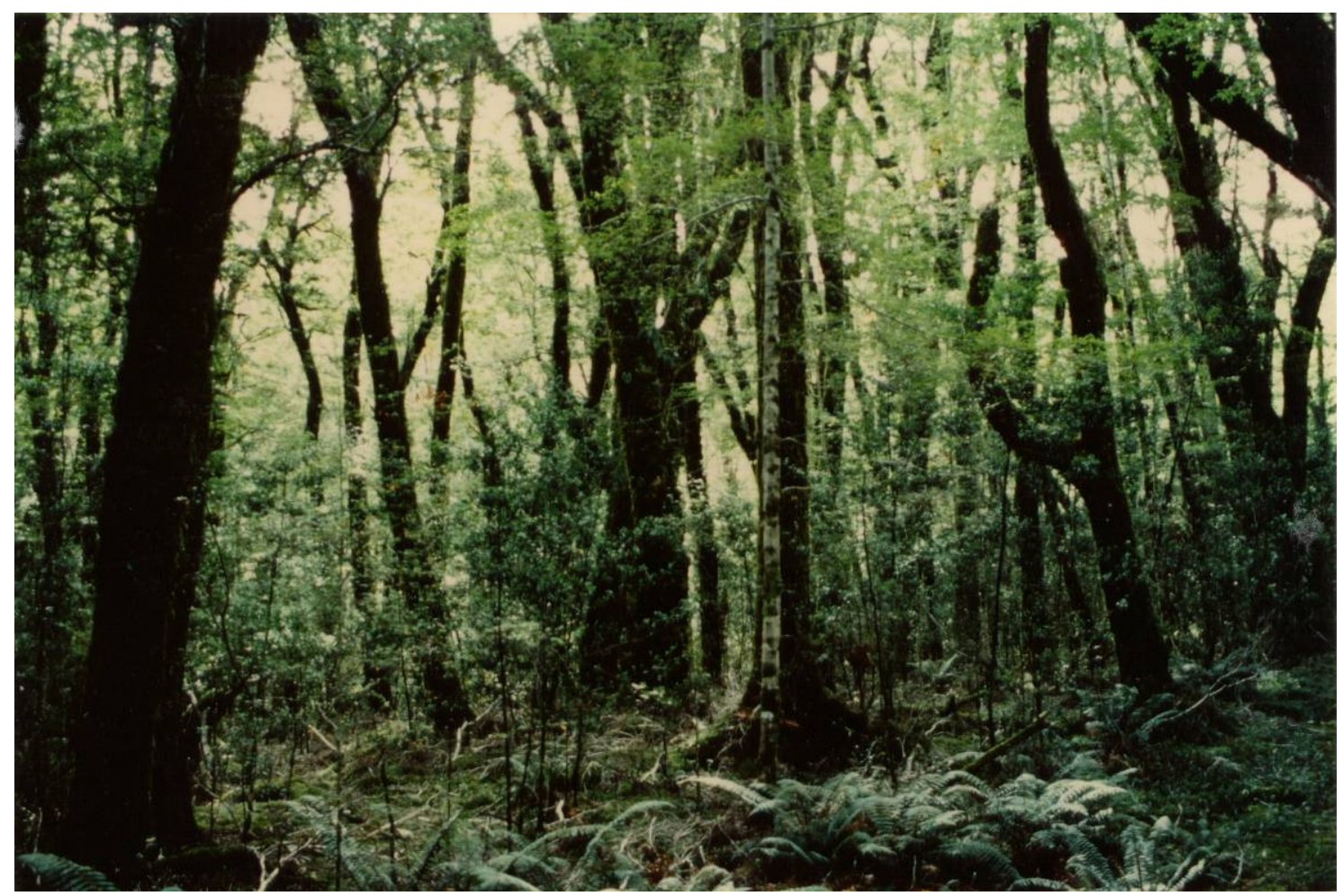

Figure 2.4: $\quad$ Silver beech dominated forest in the Knobs Flat study area. Note the understorey of mountain toatoa.

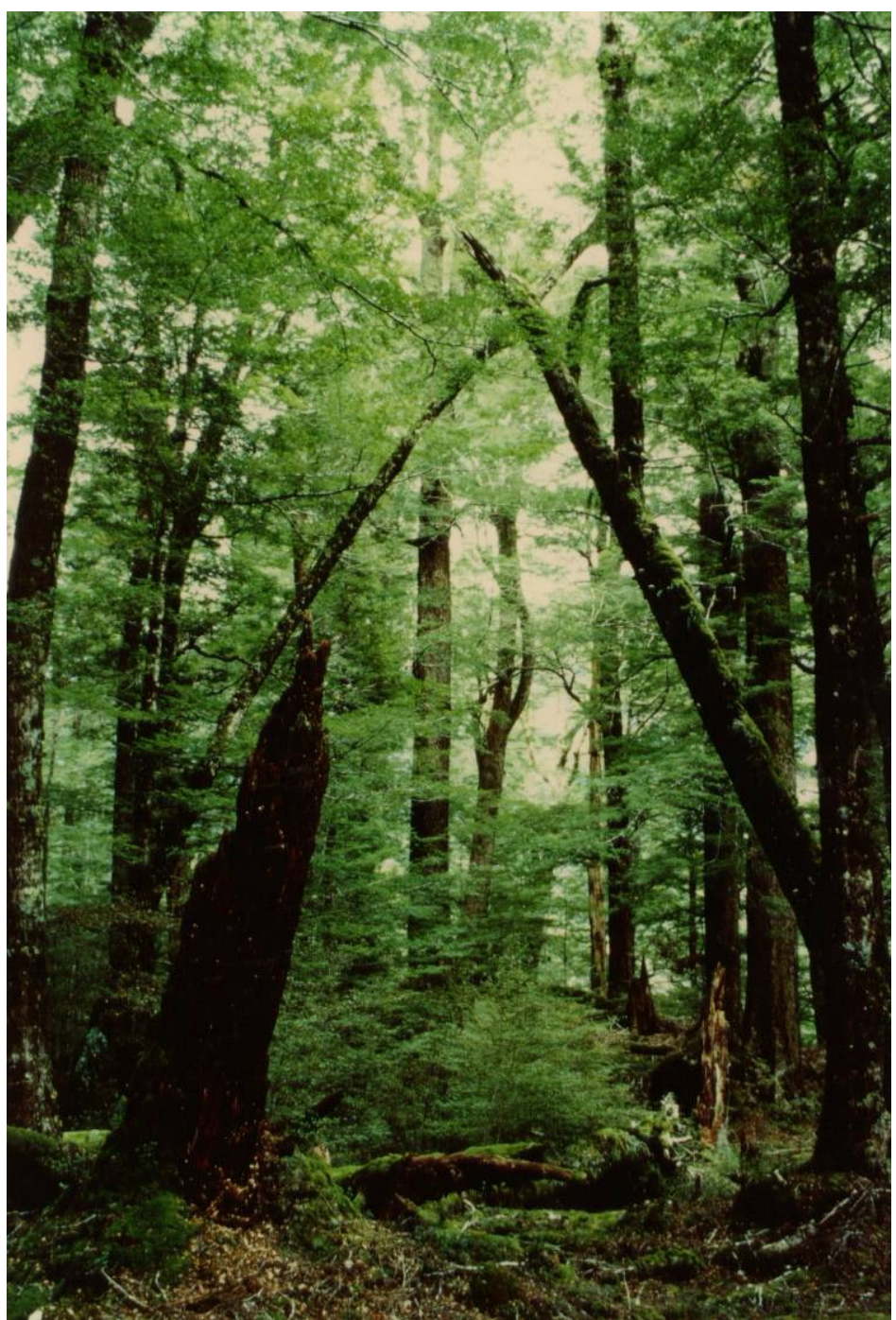

Figure 2.5: $\quad$ Red beech dominated forest in the Knobs Flat study area. 
To facilitate the mapping of bird movements, territories, and nest sites, the Knobs Flat study area was "gridded" by tying red plastic tape around trees at $50 \mathrm{~m}$ intervals (Figure 2.6). Grid points were located using compass and tape measure. The grid co-ordinates at each site were written on the plastic tape. Positions between grid points were determined during the study by taking a compass bearing from the nearest grid point and measuring the distance to it by pacing.

\section{2}

\section{PLATO CREEK}

The Plato Creek study area, is an 80 ha forested plateau at $770 \mathrm{~m}$ on the eastern side of the Eglinton valley about $7 \mathrm{~km}$ north of Knobs Flat. The forest there is of similar structure and composition to that at Knobs Flat except that the tallest trees are only about $25 \mathrm{~m}$ high.

There are no temperature or rainfall measures available for Plato Creek but the mean temperatures are probably lower than at Knobs Flat because of higher altitude, and the rainfall is probably higher because it is nearer the main divide.

Bird movements, territories and nest sites at Plato Creek were marked on a large scale map. Mapping at Plato Creek was much less accurate than at Knobs Flat. 


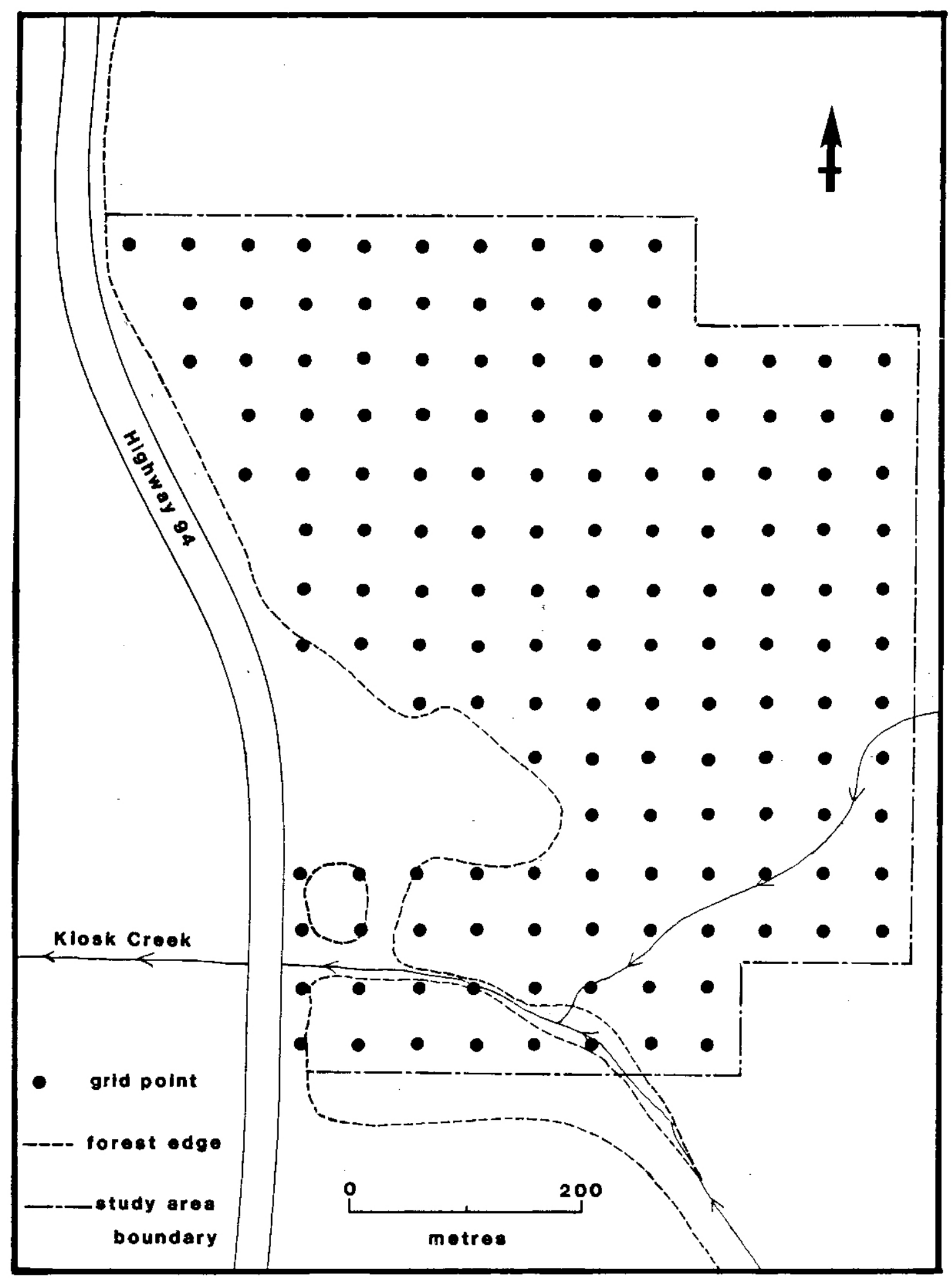

Figure 2.6: The Knobs Flat study area. 
SECTION ONE

THE YELLOWHEAD 


\section{CHAPTER 3}

\section{BEHAVIOUR OF YELLOWHEADS}

\section{1}

\section{INTRODUCTION}

Anecdotal accounts of yellowhead breeding biology by Guthrie-Smith (1936) and Soper (1976) include some information on the behaviour of the species, as does Read's (1984) more rigorous study.

All noted that during their breeding season yellowheads do not always occur in pairs. Guthrie-Smith (1936) noted that breeding pairs often associated with "... unmated though apparently marriageable youths and maidens ...", and he noted that male birds approaching a nest to feed the female were often accompanied by ". . a brace of other Canaries ..." Soper (1976) observed that families sometimes had a male and two females, and at one nest, both females incubated. Soper (1976) called these families polygamous, though Read (1984) pointed out that it was not possible to determine whether the extra female was a "nest helper" (Emlen 1984) or whether both females laid eggs. The fact that the clutch of Soper's trio was no larger than that of a pair suggests that the extra birds were helpers.

Whiteheads are also co-operative breeders, with many breeding groups consisting of more than just a pair (McLean and Gill 1988). The role of secondary birds in whitehead groups (terminology after Dow 1980) varies; some help, some are just associated with the group and some breed but do not hold their own territories (McLean et al. 1986, McLean and Gill 1988).

Co-operative breeding may occur in the brown creeper, but it is rare (McLean et al. 1987).

In all of the studies of yellowheads and whiteheads the age and sex of birds has been judged primarily by plumage, which has been shown to be unreliable (Chapter 4, Read 1984, Cunningham and Holdaway 1986, Gill and McLean 1986), and the relationship between birds has been unknown. My four year study of banded birds of known age and sex provides an opportunity to determine the nature and extent of co-operative breeding in yellowheads.

The most widely accepted model for the evolution of co-operative breeding in birds (Emlen and Vehrencamp 1983) states: 
... that when ecological constraints exist that severely limit the possibility

of personal, independent reproduction, selection will favour delayed dispersal and continued retention of grown offspring within the natal units.

Three categories of ecological constraints have been recognised:

1. shortage of territory openings;

2. shortage of mates;

3. prohibitive costs of reproduction.

Category 3 is usually associated with birds of arid and semiarid environments where breeding is difficult most of the time. For yellowheads (and whiteheads) only 1 and 2 are likely to apply.

If co-operative breeding in yellowheads did evolve in response to a shortage of territory openings or mates, it is possible that the conditions that caused its evolution no longer apply. In pre-European New Zealand for example, yellowheads may have existed in very high densities and there may have been a shortage of territory openings, thus they may have become co-operative breeders. Today yellowhead densities are mostly low and co-operative breeding may be non-adaptive or even maladaptive.

The search for explanations for the evolution of co-operative breeding has been complicated by recent work on Australian birds. It has long been known that there is a high incidence of co-operative breeding amongst Australian birds (Rowley 1969), and researchers have sought a single explanation for this (e.g. Rowley 1965, Harrison 1969). Recently Russell (1989) argued that the high incidence of co-operative breeding amongst Australasian birds is not necessarily related to recent ecological conditions, but rather to the ecological conditions encountered by their common ancestors. She argues that if we accept the new phylogeny of Sibley and Ahlquist (1985), then most of the cooperative breeders have a common ancestry. She concludes :

It would seem that co-operative breeding is yet another aspect of life history that developed early and has persisted during the evolutionary history of the passerines in Australia.

According to Sibley and Ahlquist's classification (see Holdaway 1988), yellowheads are part of the mostly Australasian parvorder Corvi which has a high incidence of cooperative breeding. Thus co-operative breeding may have developed in the yellowheads ancestors 35-40 million years ago, and conditions since then need not have favoured its development, only its persistence. 
These arguments reduce the usefulness of attempting to assess how well the ecology of yellowheads in pre-human times suits models proposed for the evolution of cooperative breeding. However, an assessment of the possibility that co-operative breeding in yellowheads is maladaptive under present conditions and has contributed to the decline of the species is still of interest.

At another level, researchers have investigated the advantages of helping behaviour to the helpers and those they help (Emlen 1982), and the covariance of helping behaviour and kinship relatedness (Brown and Brown 1980). While these topics are not closely allied to the main theme of this study (the conservation biology of yellowheads), this study has provided some information on them.

\subsection{METHODS}

Observations were made of 63 groups of yellowheads at Knobs Flat between 1984 and 1988, and 4 groups at Plato Creek during the summers of 1985-6 and 1986-7. A few observations were also made at Knobs Flat during a two week trip there in the summer of 1988-9; these are included where appropriate.

Most of the birds at Knobs Flat and some at Plato Creek were colour banded with individual combinations (for methods of capture and banding see Chapter 5).

Most observations were of necessity qualitative, or at most involved counting the frequency of behaviours.

I refer to breeding units as "groups". Groups contain a pair, or a primary pair, and secondary birds that are resident in the primary pair's territory but do not usually breed and are subordinate (terminology following Dow 1980). 


\subsubsection{Behavioural differences between males and females}

\subsubsection{Calls}

In the early stages of my study it was apparent that male and female yellowheads were easily distinguished by their calls. This was later confirmed by Harper (1988) who described yellowhead calls, their dialectal variation, and speculated on their function. Most of Harper's work was on my study populations. The following is a brief account of the differences between the calls of male and female yellowheads.

Both male and female yellowheads make the "chatter" (Harper 1988) or "rattle" (Soper 1976) call. This call is made all the time by foraging birds. However, only males were seen to utter this call loudly from the tops of trees for several minutes at a time. Harper (1988) found no detectable dialectical variation in this call.

Only male birds produce the musical and variable canary-like whistles for which the species is well known (the "male song" of Harper (1988)). Male song seems to have a strong role in territorial defence: it is often used by males in obvious territorial conflict, or males calling loudly from the tops of trees. The considerable dialectical variation that occurs in male song has been described by Harper (1988).

Only females make a buzzing call that sounds similar to one of the calls of brown creepers. Harper (1988) dubbed this call the "zurz" call. The call is not often used, and is usually associated with male song. There is some dialectical variation in this call.

\subsubsection{Territorial behaviour}

Females took a passive role in territorial disputes. Males were seen chasing other yellowheads and having "singing duels"(see section 3.3.3.4); females never did this. The greatest involvement of females in territorial interactions was when chased by males, or when making "zurz" calls when their mates were involved in "singing duels".

\subsubsection{Courtship feeding}

Female yellowheads are fed by their mates for at least two weeks before they begin laying. When being fed, female yellowheads have a distinctive wing-fluttering and begging display. 


\subsubsection{Nesting}

Only female yellowheads were observed nest-building and incubating.

Incubating females had distinctive behaviour when off the nest. They spent about 10 minutes off the nest every hour, during which time they were fed by their mates. While off the nest they foraged noticeably more quickly than their mates and when returning to their nest they flew directly to it; yellowheads rarely flew in straight lines at other times.

\subsubsection{Behavioural differences with age}

During the course of the study I banded 42 nestling or fledgling yellowheads. Six of these birds were seen in the breeding season after they fledged, four were seen two breeding seasons after they fledged and one three breeding seasons after it fledged. Of the six birds that survived at least one year, three were known to be males but the sex of the other three was unknown. Observation of these birds provided information on the behavioural development of male yellowheads, and I have speculated about the behavioural development of females.

Male, and probably female, yellowheads pass through three age classes, juvenile, immature and adult, which are recognisable by plumage and behaviour. Plumage changes are described in the next chapter.

\subsubsection{Juvenile}

From fledging until the beginning of the next breeding season, young birds remain with their parents and are fed by them and by secondary adults. Initially they are totally dependent on their parents for food, but their dependence rapidly decreases and during the winter and early spring they are fed only very rarely by their parents. The latest that juvenile birds were seen being fed by their parents was early October.

Juvenile male yellowheads attempt to make male song, though it is easily distinguishable from the song of adult males. The rhythm of their song is the same as adult male song, but the pitch is often different.

I did not see a juvenile female give the distinctive "zurz" call.

During the winter juvenile birds remained with their parents and joined other yellowhead families to form flocks of rather fluid composition (see section 3.3.4). 


\subsubsection{Immature}

By October adult yellowheads had stopped feeding juvenile birds which then became totally independent of their parents: I regard this point as the transition from juvenile to immature. They remained in this age class only until the end of the breeding season, when they moulted and their plumage and behaviour became indistinguishable from older birds.

Juvenile and immature birds were both present in the population from the beginning of December, when the first chicks fledged, until late summer when the immature birds moulted into adult plumage. Juveniles begged from their parents and were fed by them, whereas immature birds were never fed and were occasionally chased by adult birds.

Immature males attempted to sing, though their song was still distinguishable from adult song. Birds that I took to be immature were seen to give the distinctive female "zurz" call.

During the breeding season immature birds were secondaries in breeding groups (terminology of Dow 1980). Most secondaries just associated with a primary pair and were even occasionally chased by them. However, four apparently immature females paired with adult males, and one apparently paired with an apparently immature male. These pairs did not hold their own territories, but were secondary pairs. One secondary pair showed no signs of attempting to breed; one built a nest but did not lay; and two laid eggs and hatched chicks, though only one fledged chicks.

\subsubsection{Adult}

After their second moult yellowheads assumed adult plumage, and adopted adult behaviour.

During my study all adult females were members of primary pairs, but adult males were either members of primary or secondary pairs or were non-breeding secondaries. Some adult male secondaries fed the chicks of the primary pair and one even fed the primary female. Three adult male secondaries formed pairs with apparently immature females, and one pair successfully raised chicks, although these pairs did not maintain separate territories. 


\subsubsection{Behaviour during the breeding season}

By early October, all the birds in my study area at Knobs Flat had joined groups and become sedentary. Each group had a territory. The composition of groups varied: most contained a pair, but one had a lone adult male. Others included a primary pair and secondary birds that were either adult or immature birds. Two of the 63 groups studied had more than one breeding pair in the group.

The behaviour of birds during the breeding season varied with their age, sex and status.

\subsubsection{Primary adults}

The behaviour of primary pairs was identical to that described by McLean and Gill (1988) for whiteheads. That is:

The primary female built the nest and did all incubation and brooding. The primary male fed the primary female during courtship, laying, and incubation. Most feeds given during incubation were when the female was off the nest; it was rare for any bird other than the female to approach the nest before eggs hatched. Primary males accompanied ('guarded') the female during the nest building and egg-laying periods, spent long periods singing from song posts, and were occasionally involved in interactions with neighbours.

\subsubsection{Secondaries}

The behaviour of secondary birds is probably also identical in whiteheads and yellowheads though I was able to detect differences between the behaviour of adult and immature secondaries.

\subsection{Adult secondaries}

The role of adult secondaries varied, though all accompanied the female when she was off the nest. Only one was ever seen to feed a primary female. Male secondaries often sang; some sang more frequently than the primary male. Some were involved in territorial interactions with neighbours. All gave alarm calls and all mobbed intruders, such as myself, near the nest. Some adult secondaries fed nestlings and fledglings.

All but one secondary adult was male. This may not reflect a difference in the social behaviour of males and females, but simply the fact that females have a higher mortality than males (see Chapter 5). 
I made 17 nest watches of at least an hour at 7 different nests, and attempted to identify all birds visiting the nest. At nests where there were no secondary helpers females made 42.6\% (S.D. $=12.561 n=10$ ) and males $57.4 \%$ (S.D. $=12.561 n=10$ ) of visits. At nests where there was one secondary helper the primary male made 37.6\% (S.D.=9.8, $n=7$ ), the primary female $31.9 \%$ (S.D. $=7.8, n=7$ ) and the secondary male $30.4 \%$ (S.D. $=10.6, n$ $=7$ ) of visits. Two nests with three nestlings, one with a secondary helper and the other without, were each watched five times at the same time of day to determine whether nestlings were fed any more frequently in a nest with helpers than in one without. The average rate of visits to these nests was very similar (13.6 and 13.2 visits per hour) and there was no significant difference between them $(\mathrm{t}=0.169, \mathrm{df}=8, \mathrm{p}=0.87)$.

When yellowheads raised two clutches, as they usually did at Knobs Flat, the females laid their second clutches very soon after the first brood fledged and rarely fed the first fledglings. Thus, in pairs without helpers these fledglings were fed only by the primary male, who also fed the primary female when she was off the nest. Helpers that fed nestlings invariably continued to feed these chicks after they fledged and they may thus have made a considerable difference to the survival prospects of fledglings.

During my study I had only three adult secondaries of known parentage. One fed the chicks and nestlings of a pair to which it was apparently unrelated, another fed the chicks of a pair which included its father, and the third associated with a family that included its father and brother.

\subsection{Immature secondaries}

Immature male secondaries behaved in a similar manner to adult secondaries who did not help at the nest. They foraged with the primary pair, but never fed the primary female or chicks. They did not get involved in territorial interactions, but they did mob intruders.

Immature males sang, but their song was easily distinguished from that of adult males (see section 3.3.2.1) and did not seem to have any territorial function.

During the study I had no banded immature females. Most immature females probably behave in the same way as immature males. However, three females that "paired" with adult males had a lot of olive on their heads (see Chapter 4), and I suspected they were immature. None of these pairs had their own territory; they were secondaries. However, one such pair built a nest but never laid, and another hatched chicks, though none fledged. 
One apparently immature male paired with an apparently immature female and though they associated with another breeding pair they also laid and hatched chicks.

Since none of the apparently immature birds that "paired" were banded, I am not certain these birds were immature. Though immature birds did not often pair and breed, the question of whether they are capable of breeding remains in doubt.

\subsubsection{Secondary pairs}

I define secondary pairs as pairs that associated closely together and courtship fed, but which foraged with another pair to which they were apparently subordinate. The males of secondary pairs sang and apparently defended territory against birds from outside their group, but they were occasionally briefly chased by the primary male as were other secondaries.

I saw five such pairs during my study; four had adult males, but apparently immature females and the fifth had a pair of apparently immature birds.

One secondary pair showed no signs of attempting to breed, one built a nest but did not lay. Two laid eggs and hatched chicks, though only one fledged chicks. Early in the breeding season one of these pairs appeared to try and set up its own territory on the edge of the territory of the group it belonged to. However the pair was harassed by the primary male to such an extent that they did not attempt to breed and the male bird became a helper at the primary pair's nest.

\subsubsection{Territory defence}

Most territory defence was passive, with primary and secondary males singing for long periods from song-posts within their territory. Occasionally however, males from adjacent territories would have more active territorial interactions that I dubbed "singing duels". During singing duels males hopped around within a few centimetres of each other and sang very loudly. The birds did not appear to deliberately strike each other, though they were once seen to touch.

\subsubsection{Territory and mate fidelity}

Where both members of a pair were known to be alive in successive breeding seasons, they remained paired; there was no evidence of divorce. Many pairs also held similar territories in successive years: two pairs held roughly the same territories for four breeding seasons in a row and three males held roughly the same territories for five 
breeding seasons in a row, though they did not have the same mates during all this time (Figure 3.1).

When the primary males in two territories disappeared during the winter, their place was taken by a male adult helper (I and G in Figure 3.1). Thus the helpers "inherited" a mate and a territory. In one territory, both the primary male and female disappeared over a period of two years, but the territory was passed on in a recognizable chain of inheritance. In the first year the primary male (E in Figure 3.1) disappeared and his place was taken by a helper $(\mathrm{G})$. In the next year the primary female (F) disappeared and was replaced.

\subsubsection{Establishing new territories}

During my study several groups of unmarked birds apparently established new territories near the edge of my study area. However, these birds may not have moved into the area, but may have previously held territories just outside it. In the centre of my study area no new territories were established by birds from outside the study area. Two territories changed ownership in the way described in the previous section, and two male birds ( $\mathrm{K}$ and $\mathrm{J}$ in Figure 3.1) apparently annexed part of the territory of a group in which they had previously been secondaries.

For one male $(\mathrm{J})$ this process was protracted. For the first two years he was a secondary helper, in the third year he paired and tried to establish his own territory but after harassment by the primary male, reverted to his role as a helper. In the fourth year he again paired and successfully bred, though he did not hold his own territory but was a secondary breeding bird. In the fifth year the male and female were the primary pair in their own territory adjacent to the territory of the group in which the male had been a secondary.

Two other males ( $\mathrm{L}$ and $\mathrm{M}$ ) established territories adjacent, but outside, the territories in which they had been secondaries. 

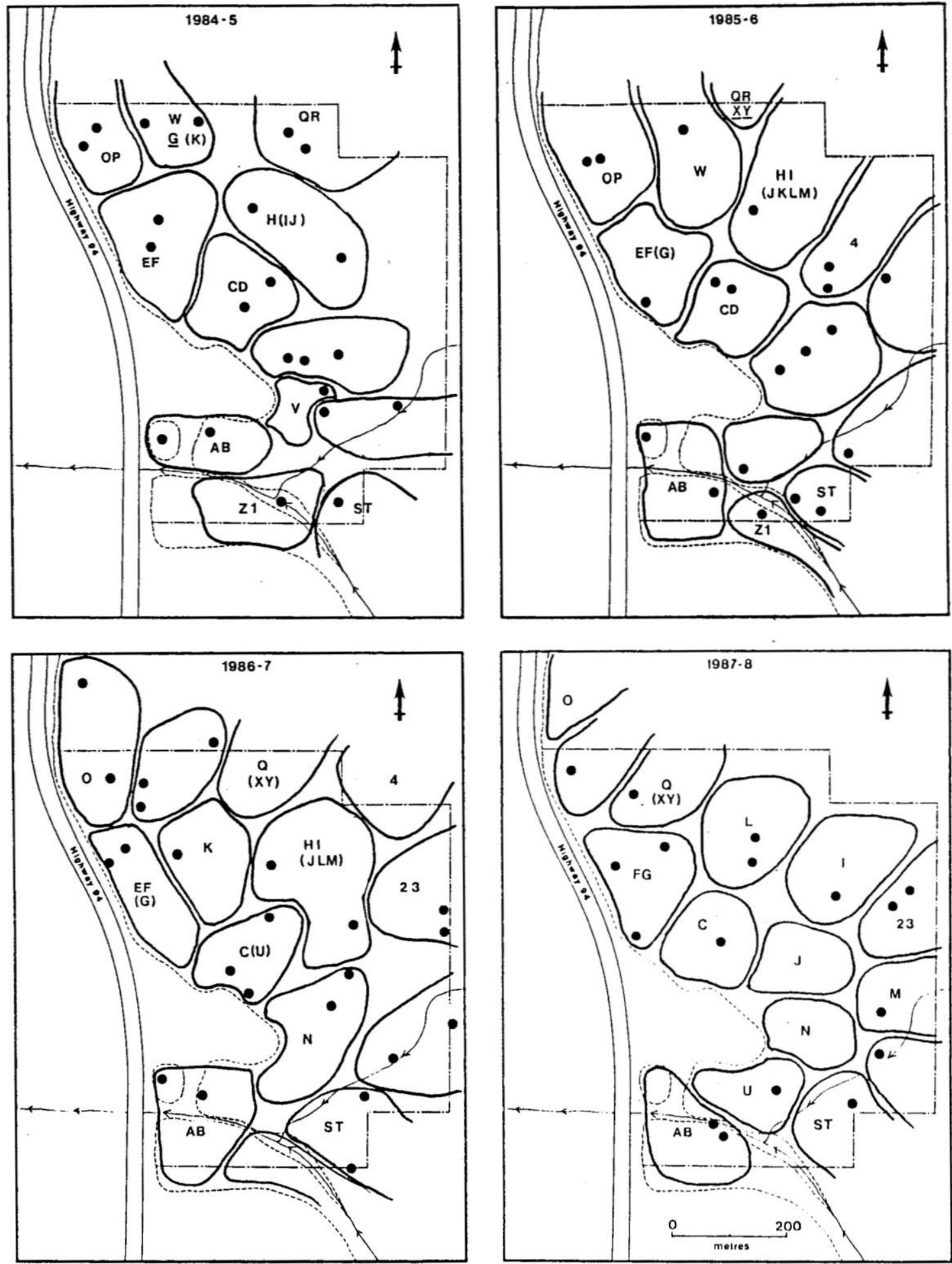

Figure 3.1: The locations of yellowhead territories and their occupants at Knobs Flat between 1984 and 1988. Thick lines represent approximate territory boundaries, black circles represent nests, and letters and numbers are banded birds. Ordinary letters and numbers represent members of primary pairs, those in brackets are secondaries, and those underlined are fledglings. 


\subsubsection{Behaviour outside the breeding season}

From the time the last chicks fledged and were mobile yellowheads ceased to defend territories, wandered outside their territories, and neighbouring groups often joined to form flocks. For most groups at Knobs Flat this change in behaviour occurred in February when their second broods were mobile. However, some pairs at Knobs Flat and all those at Plato Creek raised only one brood, and these birds started wandering in January. At Arthur's Pass, Read (1987) found that yellowheads raised only one brood and moved away from their breeding territories in January.

Yellowhead flocks at Knobs Flat were not of fixed composition but comprised "waves" of yellowheads. Flocks moved through the forest picking up groups of yellowheads which joined the flock, but later left it and returned to their summer territories. While in flocks, yellowheads moved up to $500 \mathrm{~m}$ from their summer territories, though most of their time was spent in their territory or within a few hundred metres of it.

Within the flocks families of yellowheads remained together, and adult birds occasionally fed their chicks throughout the winter. The last time chicks were seen being fed by their parents was in early October.

Some family groups at Knobs Flat and all those at Plato Creek did not follow this pattern, but behaved in the manner that Read (1987) described at Arthur's Pass. That is, once chicks fledged and were mobile families moved away from their home range and were not seen there again.

\section{4}

\section{DISCUSSION}

In the popular ornithological literature (Oliver 1955, Soper 1976, Falla et al. 1979) and in Read's (1984) study the sex of yellowheads has been determined primarily by their plumage, yet Read (1984) and Cunningham and Holdaway (1986) have found that they could not be reliably sexed on the basis of plumage. Fortunately yellowheads can easily be sexed on the basis of their calls and behaviour. Only males undertook "territorial duties" and only the females built nests and incubated. Only males made the loud song, and females made a distinctive call of their own.

The long period that young yellowheads are dependent on their parents ( 8 months) can be interpreted as evidence that yellowheads have specialised foraging behaviour . Length of parental care is positively correlated with foraging technique difficulty; the offspring of foraging specialists are generally dependent on their parents for longer than the offspring of unspecilised foragers (Morse 1980). Yellowheads certainly have a longer period of dependence than do grey warblers (Gerygone igata) (3 weeks, Falla et 
al. 1979), Chatham Island warblers (G. albofrontata) (24 days, Dennison et al. 1984), fantails (Rhipidura fuliginosa) (24 days, Powlesland 1982), yellow-breasted tits (Petroica macrocephala) (18-23 days, Kearton 1979), and robins (P. australis) (up to 50 days, Powlesland 1983).

My study suggests that yellowheads were not able to hold their own territories until they were two years old, though immature birds sometimes attempted to breed as secondaries. In my study some secondary adult males helped the primary pair by feeding the nestlings and fledglings. Soper's (1976) observation of two birds incubating a normal sized clutch suggests that females may also help by incubating as well as feeding nestlings and fledglings. Since the arrival of mammalian predators in New Zealand the presence of excess adult females is probably a rare occurrence (see Chapter 5), and female helping is consequently likely to be uncommon.

The presence of secondary breeding pairs and the possible suppression of their breeding attempts has also been observed in the whitehead (McLean et al. 1986) and in less closely related species (e.g. the splendid wren, Malurus splendens (Payne et al. 1985)).

The presence of helpers did not result in nestling yellowheads being fed more frequently, and nestlings were probably not directly advantaged by the presence of helpers. Helpers did, however, reduce the amount of effort that the primary pair put into feeding nestlings, and it may have meant that they remained in better condition during their first clutch and were more likely to attempt to raise a second.

Emlen and Vehrencamp (1983) noted that co-operative breeding often occurs in species that (1) are permanently territorial or inhabit stable or regularly predictable environments, (2) have specific ecological requirements such that suitable habitat is restricted, (3) fill all high quality habitat such that unoccupied territories are few and territory turnovers are few.

Yellowheads at Knobs Flat fitted this mould. They were not permanently territorial, but they did occupy their territories for the whole year. Their habitats were stable and they had well defined habitat requirements (see Chapter 8) such that permanent habitat was restricted. All the habitat at Knobs Flat was used by yellowheads, and territory turnovers were few. Furthermore, the commonest mechanisms of obtaining territories conferred a great advantage on co-operative breeders. Co-operative breeders sometimes inherited a territory when a member of a primary pair died, or they "budded-off" a portion of the territory. As Emlen and Vehrencamp (1983) noted "The nonbreeder must wait until it attains sufficient age, experience, and status to enable it to obtain and defend an independent territory." 
In modern New Zealand, the situation at Knobs Flat is probably rare. Most yellowhead populations today probably do not saturate their habitat and under these circumstances they may be better off attempting to breed at a young age rather than helping older pairs. However, yellowheads have substantially reduced populations throughout the South Island (Gaze 1985) and it is likely that in pre-European times many yellowhead populations would have been saturated, and co-operative breeding advantageous.

Though lack of territory openings may have led to the development, or at least maintenance, of co-operative breeding in yellowheads in pre-human New Zealand, lack of mates is likely to be a more important factor today. Stoat predation of nesting yellowheads means that there are usually more males than females (see Chapters 5 and 6) and even though there may be plenty of empty territories, males cannot find females and are forced to be secondaries in the territories of other pairs.

Co-operative breeding is probably advantageous for male yellowheads under present conditions since it is the best way for them to acquire mates and good territories. However, helping behaviour exposes helpers, especially female ones, to a greater risk of predation near the nest. Co-operative breeding may reduce unnecessary dispersal of young birds away from declining populations (I am not suggesting this advantage is adaptive) but conversely additional birds feeding nestlings might make nests more conspicuous and prone to predation.

Co-operative breeding is unlikely to significantly affect the yellowhead's plight. 


\section{CHAPTER 4}

\section{PLUMAGE AND MORPHOMETRICS OF YELLOWHEADS}

4.1 INTRODUCTION

The popular ornithological literature in New Zealand suggests that yellowheads can be sexed and aged on the basis of nape colour: yellow in adult males, but yellow tinged with olive-brown in females and juveniles (Oliver 1955, Soper 1976, Falla et al. 1979). Recently, however, Read (1984) and Cunningham and Holdaway (1986) have shown that yellowheads cannot be reliably sexed on these characteristics, though they agree that juveniles can easily be distinguished from adults.

Read (1984) noted that the proportion of bright yellow birds seen in the field dropped dramatically at about the time they moulted. He suggested that either:

1. male yellowheads had a pre-nuptial moult during which they became more yellow, and a post-nuptial moult during which they became less yellow, or

2. male yellowheads became less conspicuous after they breed.

Cunningham and Holdaway (1986) however, found that the crown feathers of adult yellowheads initially had brown tips which wore away and the bird appeared progressively more yellow.

Similar difficulties have been encountered when attempting to sex whiteheads on the basis of plumage characteristics (Gill and McLean 1986, Robertson et al. 1983).

Gill and McLean (1986), using data from this study, reported that male yellowheads were significantly larger than females, as were brown creepers and whiteheads.

Soper (1976) described some behavioural clues that might help in sexing yellowheads, not the least of which was the fact that only the females incubate. However, since Soper (1976) primarily sexed birds on the basis of their plumage, some of his behavioural clues could be misleading.

In this chapter I describe plumage and morphometric differences between yellowheads of different ages and sexes. It was not the intention of this study to investigate this in detail as it would have required repeated capture of known birds over several years and 
at different times of year. Nesting yellowheads proved easy to catch a first time, but they were very difficult to catch again. In winter, yellowheads were almost impossible to capture, as were non-breeding birds in the summer. For these reasons I have not attempted to resolve all the problems of sexing and aging yellowheads pointed out by earlier authors. This chapter's role is to provide necessary descriptive background for the main focuses of this study.

Yellowheads were studied at Knobs Flat between October 1984 and March 1988. Methods of finding nests and catching birds are described in Chapter 5. Birds were measured using the methods described by Gurr (1947). The sex of birds was judged from their behaviour (see Chapter 3).

\section{3 $\quad$ RESULTS}

\subsubsection{Adult male and female plumages}

During the study 14 breeding adult females and 15 breeding adult males were caught.

All but one of the males had some olive markings on their napes. It is clear that Soper's (1976) statement that "... the male has a completely yellow head, front and back" is incorrect. However, the males and females of 11 pairs of birds were caught at the same time and it was possible to directly compare the amount of olive of their heads. The males in 9 pairs had less olive brown on their heads than their mates. In one pair there was no detectable difference, and in another the female had less than the male. In all cases the difference between members of a pair was slight and difficult to detect except in the hand.

The difference between males and females appeared to change during the summer. The males of all 7 pairs caught in November or December had less olive than their mates. But only half of the males in the 4 pairs caught in January or February had less olive. Most adult yellowheads had commenced their moult by this time.

\subsubsection{Age-related plumages}

During the course of the study I banded 42 nestling or fledgling yellowheads. Six of these birds were seen in the breeding season after they fledged, four were seen two breeding seasons after they fledged and one, three breeding seasons after it fledged. Of the six birds that survived at least one year, three were males and the sex of the other 
three was unknown. Observation of these birds provided information on the plumage changes of male yellowheads, and I assume females are the same.

Male, and probably female, yellowheads have three recognizable age-related plumages, juvenile, immature and adult.

\subsubsection{Juvenile plumage}

Juvenile birds (i.e., birds up to one year old) (terminology of Dow 1980) are easily recognisable by the amount of olive in their plumage. All those parts of an adult yellowhead that are yellow, are yellow but flecked with olive in juveniles, and the amount of olive in the crown and nape roughly equals the amount of yellow. They also have pale legs and feet, whereas the legs and feet of older birds are black (Figure 4.1).

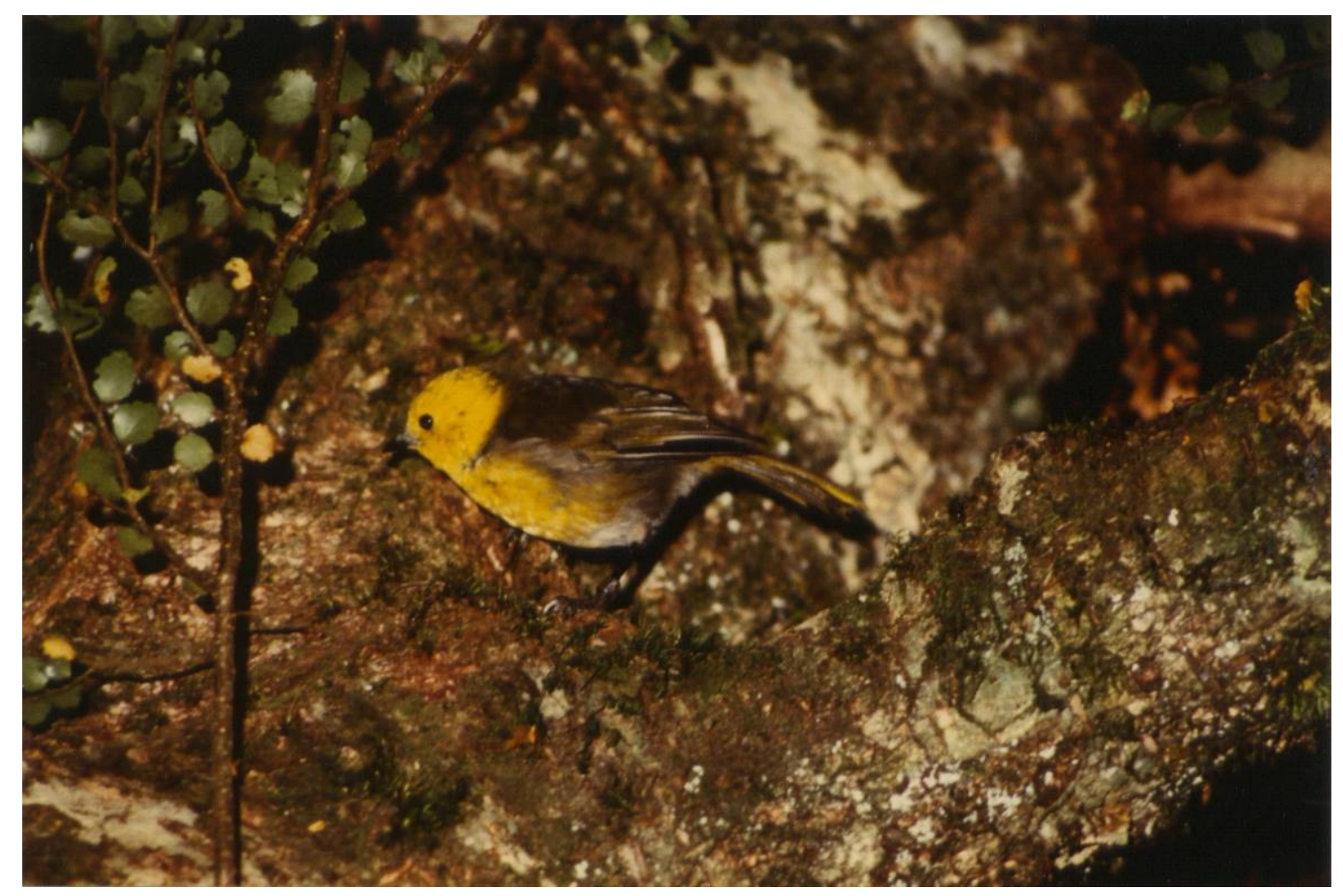

Figure 4.1: $\quad$ A juvenile yellowhead.

Juvenile birds initially have the fluffy plumage typical of young passerines, but during their first prebasic moult (terminology of Humphrey and Parkes 1959) in late summer, they lose the "fluffy" look, though they remain the same colour.

\subsubsection{Immature plumage}

Juvenile and immature birds are both present in the population from the beginning of December, when the first chicks fledge till later summer when the immature birds moult 
into adult plumage. Immatures are distinguishable by their less fluffy plumage, and darker coloured legs. Juvenile and immature plumage is the same colour.

\subsubsection{Adult plumage}

After their second moult yellowheads assume adult plumage (see frontispiece). However, two birds that were first caught in adult plumage had significantly less olive on their napes when they were recaptured a year later. It seems that adults may get progressively less olive on their napes as they get older.

\subsubsection{Morphometrics}

Measurements were made of 35 adult birds whose sex was determined from behavioural observations (Chapter 3). Males were significantly larger than females in all but one of the dimensions that I measured (Table 4.1).

\begin{tabular}{|c|c|c|c|c|c|}
\hline \multicolumn{6}{|c|}{$\begin{array}{l}\text { Comparison of measurements of male and female yellowheads. } \\
* \text { - significantly different at the } 5 \% \text { level. } \\
* * \text { - significantly different at the } 1 \% \text { level. }\end{array}$} \\
\hline & & & & & \\
\hline Measure & Male & Female & $\mathrm{t}$ & df & Probability \\
\hline Bill length (mm) & 12.73 & 11.99 & 2.69 & 33 & $0.011 *$ \\
\hline Bill depth (mm) & 5.74 & 5.20 & 3.77 & 26 & $0.001 * *$ \\
\hline Tarsus (mm) & 33.00 & 31.73 & 2.85 & 32 & $0.008 * *$ \\
\hline Mid-toe (mm) & 14.79 & 14.37 & 1.41 & 32 & 0.168 \\
\hline Wing-length(mm) & 82.48 & 76.92 & 7.26 & 32 & $0.000 * *$ \\
\hline Tail (mm) & 62.25 & 61.31 & 3.76 & 31 & $0.001 * *$ \\
\hline Weight (g) & 29.54 & 25.71 & 7.24 & 30 & $0.000 * *$ \\
\hline
\end{tabular}

Though males were significantly larger than females in all but one measure, there was overlap between the sexes in all of them: none could be used reliably to determine the sex of a bird.

I used discriminant function analysis to attempt to develop a reliable way of sexing yellowheads by measurement. Only two measures, weight and wing-length, contributed significantly to the discriminating power of the function which was given by:

Discriminant function $=0.205 \infty$ weight $+0.137 \infty$ wing-length 
When the value of the function exceeds 16.686 the bird is a male; otherwise it is a female. This function mis-classified only $6.7 \%$ of the birds of known sex for which I had measures. Classification on the basis of weight or wing-length alone would have mis-classified $10 \%$ and $12.5 \%$ respectively.

\subsubsection{Morphometrics of pairs}

Within 12 pairs of birds where both sexes were measured, the male was larger than the female for most measures (Table 4.2).

\section{Table 4.2}

The relative sizes of male and female yellowheads in 12 pairs of yellowhead.

\begin{tabular}{l|ccc} 
& Male larger & Female larger & Equal \\
\hline & & & \\
Bill length & 9 & 1 & 2 \\
Bill depth & 8 & 1 & 0 \\
Tarsus & 9 & 2 & 0 \\
Mid-toe & 10 & 1 & 0 \\
Wing length & 11 & 0 & 0 \\
Tail length & 9 & 1 & 0 \\
Weight & 11 & 0 & 0
\end{tabular}

\section{DISCUSSION}

My finding that pairs of yellowheads were more similar in plumage in late summer is consistent with Read's (1984) observation that it became more difficult to sex birds as the summer progressed.

Cunningham \& Holdaway (1986) provide a possible explanation for these observations. They found that immediately after yellowheads moult the feathers of the nape and crown have olive tips, and that the extent of the olive tip increases down the nape. They suggested that during the winter the tips wear off these feathers so that by summer the birds are olive only on their lower napes. Just before the moult female birds probably have more olive than males, but when they moult this difference is obscured by the fact that all of their head feathers have a small olive tip.

Another possible explanation is that females are usually younger than their mates. At least one adult bird that was captured twice became less olive as it aged (see later in this chapter), and the average age of males is very likely greater than females because females suffer much higher mortality (see Chapter 5). 
Further work involving repeated capture of birds of known age is required to finally sort out sex related plumage differences in yellowheads.

Gill and McLean (1986) observed in 15 whitehead pairs that males were mostly larger than females. They acknowledged that such a result could occur by chance alone, but they implied that there was assortative mating amongst whiteheads, i.e., that within a pair the male is always bigger than the female.

Given the means and variances of male and female measurements one can calculate the likelihood of a female being larger than its mate assuming they randomly assort. If, for example, male and female weights are normally distributed with means of $\bar{W}_{m}$ and $\bar{W}_{f}$ and variances of $\mathrm{S}_{\mathrm{m}}^{2}$ and $\mathrm{S}_{\mathrm{f}}^{2}$ respectively, then the difference $\left(\mathrm{D}_{\mathrm{i}}\right)$ between randomly selected males and females will also be normally distributed with a mean of $\bar{D}$ and a variance of $S_{D}^{2}$ where:

$$
\begin{aligned}
& \overline{\mathrm{D}}=\overline{\mathrm{W}}_{\mathrm{m}}-\overline{\mathrm{W}}_{\mathrm{f}} \\
& \mathrm{S}_{\mathrm{D}}^{2}=\mathrm{S}_{\mathrm{m}}^{2}+\mathrm{S}_{\mathrm{f}}^{2}
\end{aligned}
$$

When $D_{i}$ is less than 0 the female is larger than the male, and for large samples $\frac{D_{i}-\bar{D}}{S_{D}}$ is a standard normal deviate. One can thus get the probability of a female being larger than its mate from a table of areas under the normal curve (Table 4.3).

\section{Table 4.3}

The difference between measurements of male and female yellowheads and the probability of a female being larger than its mate in a randomly selected pair

\begin{tabular}{l|ccc} 
& $\begin{array}{c}\text { Average difference } \\
\text { between } \\
\text { males and females }\end{array}$ & $\begin{array}{c}\text { Standard } \\
\text { deviation of } \\
\text { difference }\end{array}$ & $\begin{array}{c}\text { Probability that } \\
\text { female is larger } \\
\text { than male }\end{array}$ \\
\hline Bill length & & & \\
Bill depth & 0.736 & 1.177 & 0.266 \\
Mid-toe & 0.535 & 0.491 & 0.138 \\
Tarsus & 0.417 & 1.148 & 0.358 \\
Wing & 1.264 & 1.698 & 0.238 \\
Tail & 5.553 & 3.225 & $0.043^{*}$ \\
Weight & 3.942 & 4.065 & 0.176 \\
& 3.827 & 2.094 & $0.034^{*}$
\end{tabular}

* Significant at the $5 \%$ level 
Given these probabilities one can calculate the expected ratio of pairs where males are larger, to pairs where females are larger, and test whether the observed results differ significantly from expected (Table 4.4). The goodness of fit test I have used is that of Sokal and Rohlf (1981) and I calculate the expected probability of the observed result and all "worse" cases by binomial expansion. There is no evidence of assortative mating in yellowheads.

\section{Table 4.4}

Test of hypothesis that male and female yellowheads randomly assort.

\begin{tabular}{|c|c|c|c|}
\hline & \multicolumn{2}{|c|}{$\begin{array}{c}\text { Ratio } \\
\text { Male large : female large }\end{array}$} & \multirow[b]{2}{*}{ Probability } \\
\hline & Observed & Expected & \\
\hline Bill length & $9: 1$ & 7.3:2.7 & 0.210 \\
\hline Bill depth & $8: 1$ & 7.8:1.2 & 0.641 \\
\hline Tarsus & $9: 2$ & $8.4: 2.6$ & 0.492 \\
\hline Mid-toe & 10:1 & $7.1: 3.9$ & 0.054 \\
\hline Wing & 11:0 & $10.5: 0.5$ & 0.620 \\
\hline Tail & $9: 1$ & $8.2: 1.8$ & 0.452 \\
\hline Weight & 10:0 & $9.7: 0.3$ & 0.708 \\
\hline
\end{tabular}

In Tables 4.5 and 4.6 I have gone through the same exercise for whiteheads using Gill and McLean's (1986) data. There is also no evidence of assortative mating in whiteheads.

\section{Table 4.5}

The difference between measurements of male and female whiteheads and the probability of a female being larger than its mate in a randomly selected pair. Data from Gill and McLean (1986).

\begin{tabular}{l|ccc} 
& $\begin{array}{c}\text { Average difference } \\
\text { between } \\
\text { males and females }\end{array}$ & $\begin{array}{c}\text { Standard } \\
\text { deviation of } \\
\text { difference }\end{array}$ & $\begin{array}{c}\text { Probability that } \\
\text { female is larger } \\
\text { than male }\end{array}$ \\
\hline Bill length & & & \\
Tarsus & 0.70 & 0.455 & 0.062 \\
Wing & 1.79 & 1.230 & 0.073 \\
Tail & 5.70 & 3.263 & $0.040^{*}$ \\
Weight & 4.70 & 4.363 & 0.157 \\
& 4.04 & 1.323 & $0.001^{* *}$
\end{tabular}




\section{Table 4.6}

Test of hypothesis that male and female whiteheads randomly assort. Data from Gill and McLean (1986)

\begin{tabular}{|c|c|c|c|}
\hline & \multicolumn{2}{|c|}{$\begin{array}{c}\text { Ratio } \\
\text { Male large : female large }\end{array}$} & \multirow[b]{2}{*}{ Probability } \\
\hline & Observed & Expected & \\
\hline Bill length & $13: 1$ & $13.1: 0.8$ & 0.787 \\
\hline Tarsus & $15: 0$ & $13.9: 1.1$ & 0.321 \\
\hline Wing & $15: 0$ & $14.4: 0.6$ & 0.540 \\
\hline Tail & $15: 0$ & $12.6: 2.4$ & 0.077 \\
\hline Weight & $15: 0$ & 14.9:0.1 & 0.984 \\
\hline
\end{tabular}

4.5 CONCLUSIONS

1. Yellowheads cannot be reliably sexed on the basis of their plumage.

2. In early summer males usually have more yellow on their heads than females, but the difference is reduced when the birds moult.

3. The amount of yellow on the heads of adult yellowheads increases as they age.

4. The apparent plumage differences between adult male and female yellowheads may not be sex related, but may be a result of females being on average younger than males.

5. Yellowheads do not assume adult plumage until they are two years old, and birds of less than two years old are easily distinguished by their dark coloured crowns and napes.

6. Immature and juvenile birds can be distinguished from each other by slight plumage differences and differences in leg colour.

7. Like whiteheads and brown creepers, the average size of male yellowheads in all dimensions is greater than female yellowheads, but there is overlap between the sexes.

8. Yellowheads do not assortatively mate. 
SECTION 2

\section{THE EFFECT OF PREDATION ON YELLOWHEADS}




\section{CHAPTER 5}

\section{PRODUCTIVITY AND MORTALITY OF YELLOWHEADS}

\section{1}

INTRODUCTION

If mammalian predators have caused a decrease in yellowhead populations throughout their range as I suggested in the introduction, then these predators must have a significant effect on yellowhead productivity or mortality or both.

Until recently, studies of yellowhead breeding biology had been anecdotal (e.g. GuthrieSmith 1936, Soper 1963), and had not attempted to assess productivity and mortality. More recently, Read (1984) studied the breeding biology of yellowheads near Arthur's Pass, and though his study was more rigorous than previous ones, he too was unable to measure productivity or mortality. He did, however, identify some factors that may significantly reduce productivity and survival. He noted that the productivity of some yellowhead populations might be much reduced because they raised only one brood a year, and he observed that recently fledged yellowheads spent much time on the ground and could be vulnerable to mammalian predation.

Yellowheads nest in holes, and though hole nesting is often thought to be a mechanism to reduce predation of nests, Alerstam and Högstedt (1981) have suggested that hole nesting may make birds more prone to mammalian predators. Gaze (1985) suggested that nest predation may be a significant factor in the yellowhead's decline.

This study aimed to measure the productivity and mortality of yellowheads and to assess:

1. the impact of mammalian predation;

2. the difference in productivity between yellowheads that raise one brood and those that raise two.

To answer these, and other questions I studied the ecology of yellowheads in part of Fiordland National Park for four years. In this chapter I report on quantitative aspects of population ecology, and in the following chapter I assess the implications of these findings for long-term trends in yellowhead populations. 
The study commenced in October 1984 and the last field work was completed in March 1988. Each summer during the period from late October till early March all the groups of yellowheads in the Knobs Flat study area were regularly monitored. In addition, a few pairs which nested near the boundaries of the study area were monitored. Nests were searched for and regularly checked, and where possible, birds were caught, measured and banded with a unique combination of numbered metal and coloured plastic bands.

At Plato Creek the progress of yellowhead breeding was monitored only in the summers of 1985-6 and 1986-7. The difficulties of access and low number of birds at Plato Creek meant that very little information on yellowhead breeding was obtained.

Observation of birds during winter was confined to two, two-week visits to Knobs Flat in 1985 and 1987 when the behaviour and locations of banded birds were recorded.

During the summer of 1988-1989, i.e., the year following the completion of this study, I visited Knobs Flat for a week and counted the number of birds within the study area. During this time I did not attempt to find nests but from behavioural clues I was able to determine which of the birds were breeding. The results of this visit are included where appropriate.

\subsubsection{Finding nests}

Nests were found by following each group of birds for at least an hour every few days. Nest sites were marked with red plastic tape and the position of the nest recorded.

\subsubsection{Monitoring nests}

Once located, nests were inspected periodically. Each time the nest was inspected, the number of eggs and chicks, the presence of adults at the nest, and any sign of predation or interference was recorded. Yellowhead nests are often deep within holes and it was often necessary to use a small mirror and torch to see the nest contents.

\subsubsection{Climbing up to nests}

Yellowheads nest in holes in trees and most nests are high off the ground (see Chapter 10). Nests were reached by placing a rope over a branch above the nest hole, and ascending and descending the rope using equipment and techniques developed by cavers and mountaineers (Montgomery 1977). 
Ropes were placed over branches above nests by firing a spherical $57 \mathrm{~g}$ lead sinker attached to braided polyester fly-fishing backing line ( $9 \mathrm{~kg}$ breaking strain), over the branch using a commercially available sporting catapult. The line was dispensed from a fishing reel strapped to the wrist. Once the fishing line was over a suitable branch, a stronger braided polyester line was pulled over the branch and that was in turn used to pull over an $11 \mathrm{~mm}$ diameter caving rope. One end of the rope was secured to another tree and the free end was climbed.

After the nest was inspected the rope was replaced with braided polyester which was left in place to facilitate the next nest inspection (placing the rope in the tree for the first time took up to two hours).

Using these techniques, nests up to $30 \mathrm{~m}$ off the ground could be regularly inspected.

\subsubsection{Catching, banding and measuring birds}

\subsubsection{Catching adults}

Adult yellowheads were caught in mist nets erected up to $25 \mathrm{~m}$ above the ground using techniques developed by Humphrey et al. (1968) and Whitaker (1972).

Initially, yellowheads were attracted into the nets by playing tape-recordings of their calls near the nets, but by the second year of the study most of the yellowheads showed only a slight response to taped calls. From the second year, yellowheads were caught by erecting mist nets near their nests, where they were found to be more responsive to taped calls. The nets were erected when large chicks were in the nest and their parents were making frequent feeding trips. When both adults were in or near the nest hole and had just fed the chicks, taped calls were played from the side of the net farthest from the nest and in most cases the male bird, and sometimes the female, would fly into the net.

\subsubsection{Catching fledglings and nestlings}

Some nest holes were large enough for nestlings to be removed by hand for banding, but most were not. Some nestlings in small holes could be removed by passing a small noose around their bodies and pulling them out, but even using this technique most nestlings could not be removed. 
The timing of banding nestlings was important since they might fledge prematurely if handled too close to their normal fledging time. This problem was anticipated and nestlings were banded at least four days before they were due to fledge.

Some fledglings were caught and banded on the day they fledged. Recently fledged yellowheads have only limited powers of flight and some fall to the ground when they first leave the nest. They eventually climb up into the canopy, but until they do so, they are easily caught.

\subsubsection{Banding}

Each yellowhead caught was banded with a unique combination of bands, including one size C numbered metal band, and two split-ring, coloured, plastic bands.

\subsubsection{Measurements}

Each bird caught was weighed, measured and a description of its plumage, and any evidence of moult recorded. The following measurements were taken; tarsus length, tail length, wing length, bill length, width and depth, and mid-toe and claw length.

\subsubsection{Sex and age of birds}

Birds were classed as either juvenile, immature or adult on the basis of their plumage (see Chapter 4). The sex of birds was determined from their behaviour (see Chapter 3) either immediately before they were caught or by subsequent observation.

\section{RESULTS}

\subsubsection{Density}

Table 5.1 shows the numbers of birds in each of the various age, sex, and breedingstatus classes in November, the beginning of each breeding season, at Knobs Flat. 


\begin{tabular}{|c|c|c|c|c|c|c|c|c|}
\hline \multirow[b]{5}{*}{ Year } & \multicolumn{7}{|c|}{ Table 5.1} & \multirow[b]{5}{*}{ Total } \\
\hline & \multicolumn{7}{|c|}{ Numbers of yellowheads in November at Knobs Flat } & \\
\hline & \multicolumn{4}{|c|}{ Adult } & \multirow{2}{*}{\multicolumn{3}{|c|}{ Immature }} & \\
\hline & \multicolumn{2}{|c|}{ Breeding } & \multicolumn{2}{|c|}{ Non-breeding } & & & & \\
\hline & $\mathrm{m}$ & $\mathrm{f}$ & $\mathrm{m}$ & $\mathrm{f}$ & $\underline{\underline{m}}$ & $\mathrm{f}$ & unk. & \\
\hline 1984 & 12 & 12 & 0 & 0 & 3 & 0 & 1 & 28 \\
\hline 1985 & 13 & 13 & 3 & 0 & 4 & 1 & 7 & 41 \\
\hline 1986 & 12 & 12 & 6 & 0 & 3 & 0 & 1 & 34 \\
\hline 1987 & 11 & 11 & 5 & 3 & 0 & 0 & 1 & 31 \\
\hline 1988 & 7 & 7 & 4 & 0 & 4 & 0 & 1 & 23 \\
\hline
\end{tabular}

The low numbers of birds in 1988 resulted from high mortality and low breeding success in the previous summer, in which there was a stoat plague (see section 5.3.2.11).

A notable feature of the 1987 breeding season was that only 11 pairs bred, though there were sufficient adults for 14 pairs. This supports behavioural observations which suggest that territorial interactions limit the number of birds that breed (see Chapter 3 ).

\subsubsection{Productivity}

\subsubsection{Number of clutches}

Yellowheads at Knobs Flat reared a maximum of two broods a year, but where nests failed, some pairs attempted three. Although apparently capable of raising two broods, only $77 \%$ of the breeding pairs investigated in this study attempted to do so. The proportion of pairs that nested more than once each year varied from 85 to $67 \%$ (Table 5.2) but the differences were not significant $\left(\chi^{2}=2.298\right.$, df $\left.=3, p=0.51\right)$. 
Table 5.2

Proportion of pairs that attempted more than one clutch.

\begin{tabular}{l|ccc} 
& $\begin{array}{c}\text { No. } \\
\text { pairs } \\
\text { studied }\end{array}$ & $\begin{array}{c}\text { No. } \\
\text { pairs } \\
\text { 2+ clutches }\end{array}$ & Percent \\
\hline $84-85$ & & 11 & $85 \%$ \\
$85-86$ & 13 & 10 & $67 \%$ \\
$86-87$ & 15 & 12 & $86 \%$ \\
$87-88$ & 14 & 4 & $67 \%$ \\
& 6 & 37 & $77 \%$
\end{tabular}

The four pairs of yellowheads that bred at Plato Creek laid only one clutch each, and after nest failures they did not renest.

\subsubsection{Timing of breeding}

Active yellowhead nests were found between early October and early March (Figure 5.3). At Knobs Flat nesting activity was initially synchronised with the incubation of most first clutches starting in the first few days of November. Hatching, fledging and laying of second clutches was not so well synchronised, since some failed and were soon replaced, whereas others produced chicks before another was laid. None-the-less, there are two peaks of breeding activity, the first corresponding with the laying of first clutches and the second, less pronounced peak, corresponding with second clutches laid after successful first ones. 


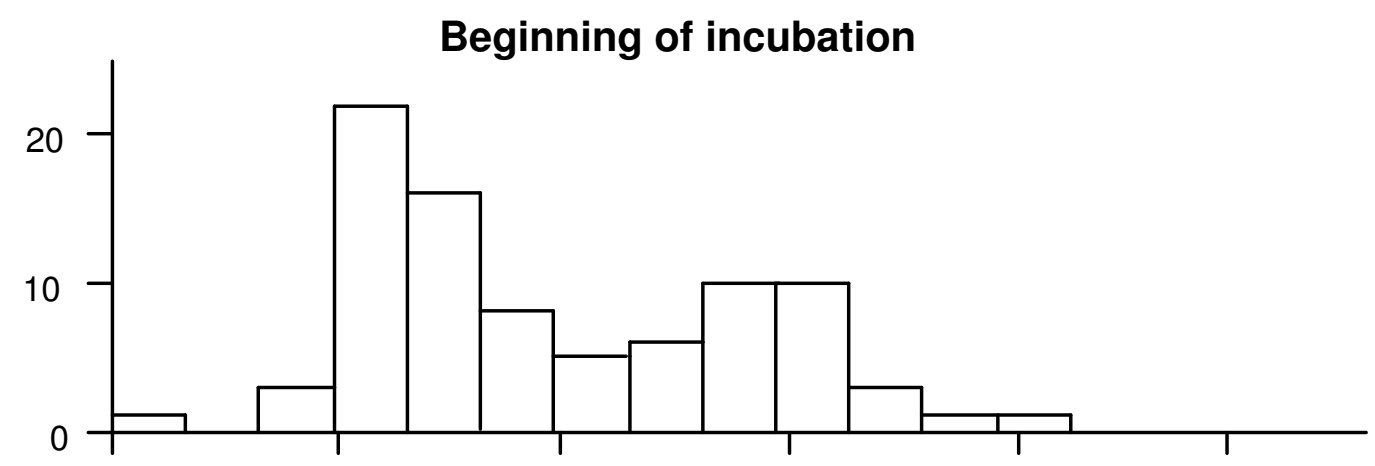

Hatching

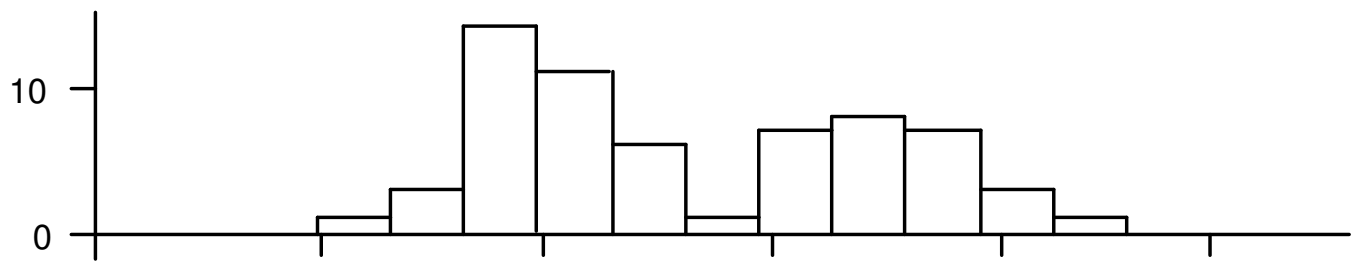

Fledging

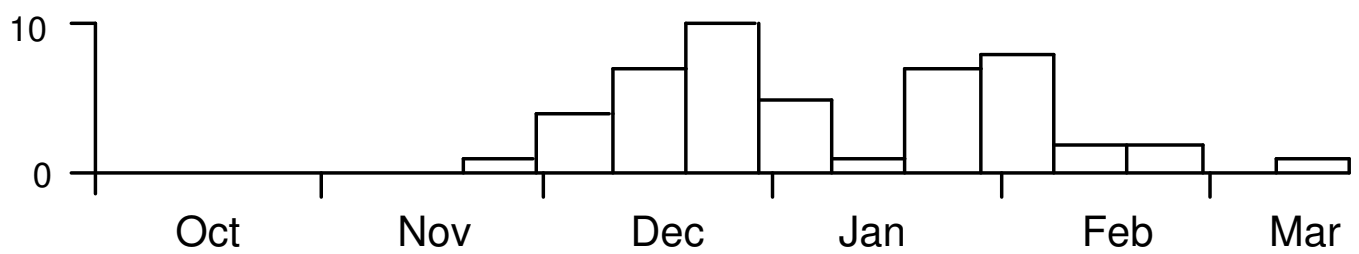

Figure 5.3: The timing of the beginning of incubation, hatching and fledging of yellowheads at Knobs Flat (all years combined).

There was some variation in the timing of breeding between the four years of the study (Table 5.3). I compared the beginning of incubation of first nesting attempts (measured in days since the end of September) for the four years using the Kruskal-Wallis test, and found that the variation was not significant $(\mathrm{H}=3.197, \mathrm{df}=3, \mathrm{p}>0.10)$

\section{Table 5.3}

Variation in the timing of breeding between years at Knobs Flat.

\begin{tabular}{l|rrrr} 
& \multicolumn{4}{|c}{ Date of beginning of incubation } \\
Year & Median & Earliest & Latest & $n$ \\
\hline & & & & \\
1984 & $5 \mathrm{Nov}$ & 9 Oct & $11 \mathrm{Dec}$ & 12 \\
1985 & $13 \mathrm{Nov}$ & 30 Oct & $25 \mathrm{Dec}$ & 12 \\
1986 & $7 \mathrm{Nov}$ & $26 \mathrm{Oct}$ & $22 \mathrm{Nov}$ & 12 \\
1987 & $10 \mathrm{Nov}$ & 30 Oct & $22 \mathrm{Nov}$ & 12
\end{tabular}


Most pairs started laying in the first few days of November and fledged their last clutch by mid February, giving a breeding season of about $3^{1} / 2$ months. The longest breeding season of any pair was from about 25 October, when they laid their first clutch, till 9 March when their second clutch fledged; a period of $4^{1} / 2$ months.

At Plato Creek the timing of only three nests was known. The dates at which incubation began were 10 November, 8 and 12 December.

\subsubsection{Nest building}

Nests were built by females though males invariably followed their mates back and forward to the nest while it was being built. Nests were built in bursts; females made several trips to their nests with material, but would then forage for several hours before resuming nest construction.

18 nests were found while they were being built, but I was unable to determine how long the nests had been under construction before they were found. The maximum period over which a nest was observed being built was 10 days, and the maximum time between finding a nest under construction and the first egg being laid was 12 days.

Three females were observed each building two nests at the same time, though in each case they only finished and used one of them.

Some females built, and sometimes laid, in their second nests before their first brood had fledged: one female began incubating her second clutch only three days after the first had fledged.

\subsubsection{Laying}

No special attempt was made to record the time it took birds to lay clutches, but it was apparent at four nests that eggs were sometimes laid at daily intervals and at other times at two-day intervals. Incubation started with the laying of the last egg.

\subsubsection{Incubation period}

To minimise disturbance during laying and hatching, attempts to determine the length of the incubation period were made at only four nests, which were checked daily from the fifteenth day of incubation. The beginning of incubation was regarded as being halfway between the time when a nest containing eggs was last checked but found not to be incubated, and the first check when it was. Similarly the end of incubation was half- 
way between the last check when no eggs had hatched and the first check when some had. The average incubation period was about 20 days (Table 5.4).

\section{Table 5.4}

Incubation period at four yellowhead nests.

\begin{tabular}{l|l} 
Nest & Incubation period \\
\hline $2 / 26$ & 21 days 18 hours \\
$3 / 7$ & 19 days I hour \\
$2 / 29$ & 19 days 19 hours \\
$3 / 2$ & 20 days 7 hours \\
Mean & 20 days 5 hours
\end{tabular}

\subsubsection{Nestling period}

To minimise disturbance during hatching and fledging, attempts to determine the length of the nestling period were made at only three nests, which were checked daily from the fifteenth day after hatching. In all three cases the nests were checked when hatching was in progress and the time of this check was regarded as the beginning of the nestling period. The end of the nestling period was the time at which chicks were seen leaving the nest in one case, and half-way between the last check when chicks were present and the first check when they had gone, in the other two. The average nestling period was about $22^{1} /{ }_{2}$ days (Table 5.5).

\section{Table 5.5}

Nestling period at three yellowhead nests.

\begin{tabular}{l|l} 
Nest & Nestling period \\
\hline $1 / 8$ & 22 days 13 hrs \\
$1 / 4$ & 22 days 21 hours \\
$3 / 10$ & 21 days 21 hours \\
Mean & 22 days 11 hours
\end{tabular}




\subsubsection{Clutch size}

Since some eggs were lost during incubation, only clutches found within four days of being laid were considered in this analysis. Clutches varied from 1 to 4 eggs (Figure $5.4)$, with a mean of $3.06(n=49, \mathrm{SD}=0.78)$.

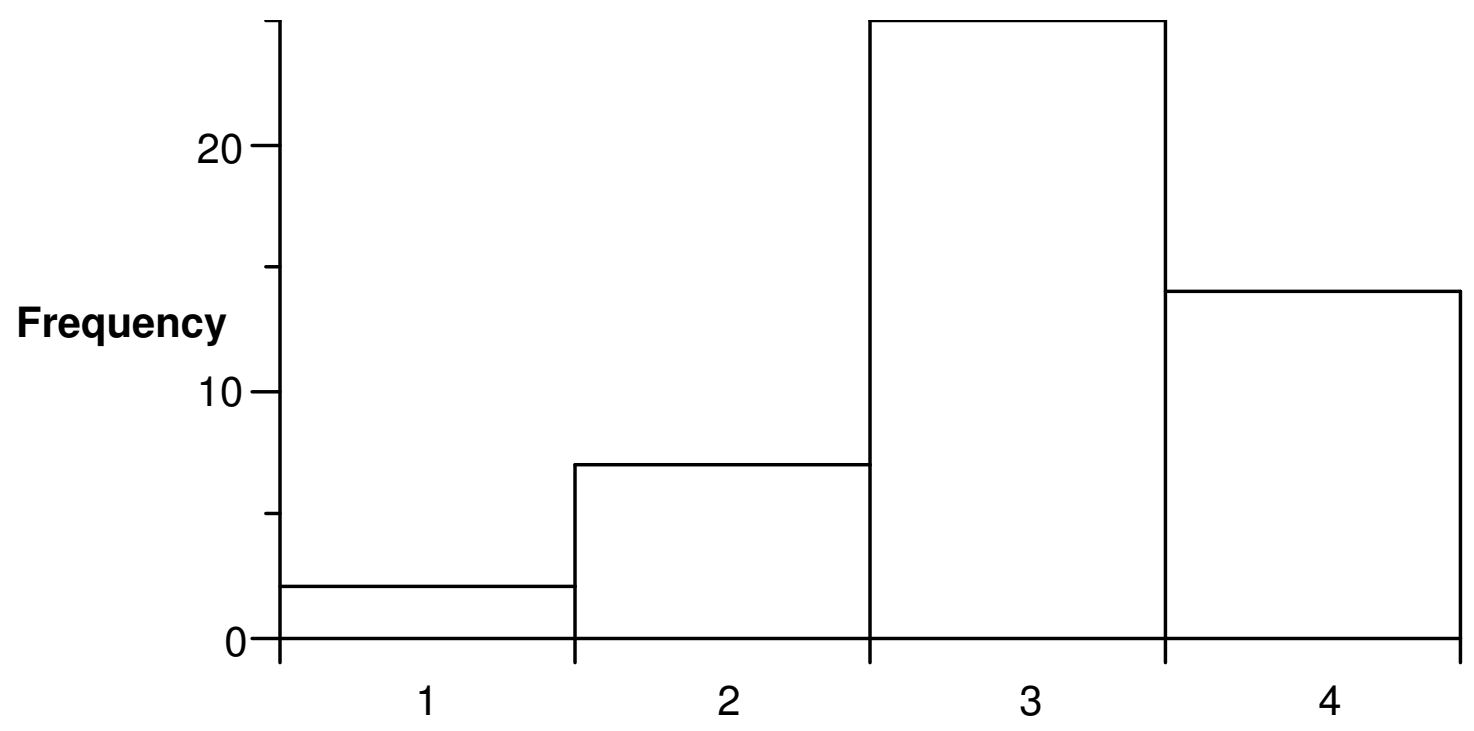

\section{Clutch size}

Figure 5.4: $\quad$ The frequency distribution of yellowhead clutch size.

Kruskal-Wallis and Mann-Whitney tests were used to test for significant variation in clutch size. Clutch sizes did not vary significantly between first and subsequent clutches (Table 5.6), or between months (Table 5.7), but did vary significantly between years (Table 5.8).

\section{Table 5.6}

Clutch sizes of first and subsequent clutches and Mann-Whitney test

\begin{tabular}{r|ccc} 
& Mean & SD & $n$ \\
\hline First clutch & 3.07 & 0.64 & 30 \\
Subsequent clutches & 3.05 & 0.97 & 19
\end{tabular}

$$
\begin{aligned}
Z & =0.395 \\
\text { Probability } & =0.69 \text { (not significant) }
\end{aligned}
$$


Table 5.7

Variation in clutch size between months and Kruskal-Wallis test

\begin{tabular}{l|ccr} 
Month & Mean & SD & $n$ \\
\hline & & & \\
October & 3.40 & 0.89 & 5 \\
November & 3.04 & 0.72 & 26 \\
December & 3.22 & 0.83 & 9 \\
January & 2.78 & 0.83 & 9
\end{tabular}

Degrees of freedom $=3$

Probability $>0.10$ (not significant)

Table 5.8

Variation in clutch size between years and Kruskal-Wallis test

\begin{tabular}{l|rrr} 
Year & Mean & SD & $n$ \\
\hline & & & \\
1984 & 3.11 & 0.93 & 9 \\
1985 & 2.69 & 0.79 & 16 \\
1986 & 3.54 & 0.52 & 13 \\
1987 & 3.00 & 0.63 & 11
\end{tabular}

$\mathrm{H}=9.66$

Degrees of freedom $=3$

Probability $<0.025$ (significant)

\subsubsection{Hatching success}

Of 87 nests in which eggs were laid at Knobs Flat 62, (71\%) had at least 1 egg hatch. In 36 nests, the number of eggs that were laid was known and the number of nestlings was counted within two days of hatching. In these nests 58 (54\%) of the 107 eggs hatched. There was no significant yearly variation in the hatching success of clutches and only questionable significance in the hatching success of eggs (Table 5.9). 


\begin{tabular}{|c|c|c|c|c|c|}
\hline \multicolumn{6}{|c|}{ Table 5.9} \\
\hline \multicolumn{6}{|c|}{ Yearly variation in hatching success at Knobs Flat } \\
\hline Year & $\begin{array}{l}\text { No. clutches } \\
\text { laid }\end{array}$ & $\begin{array}{c}\% \\
\text { hatched } \\
\end{array}$ & $\begin{array}{l}\text { No. clutches } \\
\text { counted }\end{array}$ & $\begin{array}{l}\text { No. eggs } \\
\text { counted }\end{array}$ & $\begin{array}{c}\% \\
\text { hatched } \\
\end{array}$ \\
\hline $1984-5$ & 24 & 71 & 5 & 14 & 29 \\
\hline $1985-6$ & 22 & 68 & 12 & 31 & 68 \\
\hline $1986-7$ & 26 & 65 & 10 & 36 & 42 \\
\hline $1987-8$ & 15 & 87 & 9 & 26 & 69 \\
\hline \multirow[t]{2}{*}{ Total } & 87 & 71 & 36 & 107 & 54 \\
\hline & $\begin{array}{c}\chi^{2} \\
\mathrm{df} \\
\mathrm{p}\end{array}$ & $\begin{array}{l}2.28 \\
3 \\
0.52\end{array}$ & & $\begin{aligned} \chi^{2} & =7.50 \\
\mathrm{df} & =3 \\
\mathrm{p} & =0.06\end{aligned}$ & \\
\hline
\end{tabular}

\subsubsection{Fledging success}

Of 62 nests that hatched some eggs at Knobs Flat, 48 (77\%) had at least one chick fledge. In 25 nests where the nestlings were counted soon after hatching, there were 66 nestlings of which $42(64 \%)$ fledged. There was significant variation between years in both these statistics (Table 5.10), caused by low fledging success in the summer of 1987-8 when there was a high rate of stoat predation (see section 5.3.2.11).

Table 5.10

Yearly variation in fledging success at Knobs Flat

\begin{tabular}{|c|c|c|c|c|c|}
\hline Year & $\begin{array}{c}\text { No. broods } \\
\text { hatched }\end{array}$ & $\begin{array}{c}\% \\
\text { fledged } \\
\end{array}$ & $\begin{array}{l}\text { No. broods } \\
\text { counted }\end{array}$ & $\begin{array}{l}\text { No. chicks } \\
\text { counted }\end{array}$ & $\begin{array}{c}\% \\
\text { fledged } \\
\end{array}$ \\
\hline 1984-5 & 17 & 82 & 3 & 10 & 80 \\
\hline $1985-6$ & 15 & 93 & 8 & 20 & 80 \\
\hline 1986-7 & 17 & 82 & 5 & 15 & 67 \\
\hline $1987-8$ & 13 & 46 & 9 & 21 & 38 \\
\hline \multirow[t]{2}{*}{ Total } & 62 & 77 & 25 & 66 & 63 \\
\hline & $\begin{array}{r}\chi^{2} \\
\mathrm{df} \\
\mathrm{p}\end{array}$ & $\begin{array}{l}9.916 \\
3 \\
0.02\end{array}$ & \multicolumn{3}{|c|}{$\begin{aligned} \chi^{2} & =9.451 \\
\mathrm{df} & =3 \\
\mathrm{p} & =0.02\end{aligned}$} \\
\hline
\end{tabular}




\subsubsection{Nesting success}

Of 87 clutches laid at Knobs Flat, 48 (55\%) fledged at least one chick. In 46 nests where the eggs were counted soon after laying, there were 140 eggs from which 44 chicks fledged $(31 \%)$. There was no significant variation in nesting success between years (Table 5.11).

\begin{tabular}{|c|c|c|c|c|c|}
\hline \multirow[b]{3}{*}{ Year } & \multicolumn{4}{|c|}{ Table 5.11} & \multirow[b]{3}{*}{$\begin{array}{c}\% \\
\text { fledged }\end{array}$} \\
\hline & \multicolumn{4}{|c|}{ Yearly variation in nesting success at Knobs Flat } & \\
\hline & $\begin{array}{c}\text { No. clutches } \\
\text { laid } \\
\end{array}$ & $\begin{array}{c}\% \\
\text { fledged } \\
\end{array}$ & $\begin{array}{l}\text { No. clutches } \\
\text { counted }\end{array}$ & $\begin{array}{l}\text { No. eggs } \\
\text { counted } \\
\end{array}$ & \\
\hline 1984-5 & 24 & 58 & 9 & 28 & 30 \\
\hline 1985-6 & 22 & 64 & 14 & 36 & 44 \\
\hline 1986-7 & 26 & 54 & 13 & 46 & 26 \\
\hline $1987-8$ & 15 & 40 & 10 & 40 & 23 \\
\hline Total & 87 & 55 & 46 & 140 & 31 \\
\hline & $\begin{array}{r}\chi^{2} \\
\mathrm{df} \\
\mathrm{p}\end{array}$ & .149 & & $\begin{aligned} \chi^{2} & =5.0 \\
\text { df } & =3 \\
\mathrm{p} & =0.1\end{aligned}$ & \\
\hline
\end{tabular}

There was no significant difference between the number of chicks fledged per nest by first or subsequent nesting attempts (Table 5.12).

\section{Table 5.12}

Number of chicks produced by first and subsequent nesting attempts.

\begin{tabular}{l|ccc} 
& Mean & SD & N \\
\hline & & & \\
First nest & 1.08 & 1.239 & 50 \\
Subsequent nest & 1.20 & 1.381 & 40
\end{tabular}

(Mann-Whitney) $\mathrm{Z}=0.348$

Probability $=0.728$

\subsubsection{Causes of nest loss}

Table 5.13 summarises the reasons for, and timing of, nest failure at 39 nests that failed to produce chicks at Knobs Flat. 
Table 5.13

Nest failures at Knobs Flat

\begin{tabular}{|c|c|c|c|c|c|c|}
\hline \multirow[b]{2}{*}{ Year } & \multicolumn{2}{|c|}{ Time of failure } & \multicolumn{4}{|c|}{ Reason for failure } \\
\hline & Egg & Chick & Unknown & Accident & Cuckoo* & Predator \\
\hline 1984-5 & 6 & 4 & 6 & 2 & 2 & 0 \\
\hline $1985-6$ & 6 & 2 & 7 & 0 & 1 & 0 \\
\hline 1986-7 & 8 & 4 & 11 & 0 & 1 & 0 \\
\hline 1987-8 & 2 & 7 & 3 & 0 & 0 & 6 \\
\hline
\end{tabular}

During the first three years of the study, there was a consistent pattern of nest loss. Most losses occurred at the egg stage and the cause was mostly unknown. Where it was known, it was not caused by predation, but either by nest parasitism by long-tailed cuckoos, or "accidents" (one nest flooded during a storm and one nest was crushed when the tree it was in fell down). An interesting feature at seven failed nests in the first three years, is that before the nests were abandoned some, but not all, of the eggs disappeared. I suspect that at least some of these eggs were taken by long-tailed cuckoos, and nest robbing and parasitism by long-tailed cuckoos is discussed more fully in Chapter 7.

In the last year of the study, the pattern of nest loss was quite different; most were preyed upon and most nest failures occurred during the chick stage rather than the egg stage.

At four of the six nests that were preyed upon there were remains of adults, chicks or eggs left in the nests, and at one nest a long-tailed cuckoo was seen removing the chicks from the nest and eating them. Two nests were found empty but otherwise undisturbed. The only clue that they had been preyed upon was that the females disappeared at the same time the nests failed. In previous years no females disappeared during the breeding season and this fact combined with the obvious predation at other nests from which females also disappeared led me to conclude that the contents of the two empty undisturbed nests were also preyed upon.

At one nest that was preyed upon there were stoat droppings in and around the nest hole, and at all the other nests, except the one taken by a long-tailed cuckoo, there was circumstantial evidence that stoats were the predator. None of the nests contained the egg shell fragments, or gnawed bones characteristic of rodent predation (Moors 1978). 
Stoats are the only other predatory mammals in the Eglinton Valley that are small enough to enter yellowhead nests.

The high incidence of stoat predation in the last year of the study was correlated with a "stoat plague" induced by a beech mast and increased numbers of mice (King 1983). I counted both live and dead stoats seen on the road between Te Anau and Knobs Flat and in my study area between November and February each summer. Since I spent approximately the same amount of time in my study area each summer and travelled the road the same number of times, these counts provide a rough index of stoat abundance (Table 5.14).

\section{Table 5.14}

Stoat counts at Knobs Flat between November and February for four summers.

\begin{tabular}{l|cccc} 
Year & $\begin{array}{c}\text { In study } \\
\text { area }\end{array}$ & $\begin{array}{c}\text { Live on } \\
\text { road }\end{array}$ & $\begin{array}{c}\text { Dead on } \\
\text { road }\end{array}$ & Total \\
\hline & & & & \\
$1984-5$ & 0 & 0 & 0 & 0 \\
$1985-6$ & 0 & 0 & 0 & 0 \\
$1986-7$ & 0 & 0 & 0 & 0 \\
$1987-8$ & 2 & 2 & 3 & 7
\end{tabular}

\subsubsection{Productivity}

I measured the productivity of yellowheads at Knobs Flat by counting the number of fledglings produced by each pair of birds each breeding season (Table 5.15). Productivities from first broods are presented to give an indication of the likely productivity if yellowheads nested only once a year, instead of twice.

\section{Table 5.15}

Yellowhead productivity at Knobs Flat, estimated by counting the number of fledglings produced by each pair

\begin{tabular}{l|cc|c} 
& \multicolumn{2}{|c|}{ Fledglings/pair } & \\
Year & 1st clutch & all clutches & No. of pairs \\
\hline & & & \\
$1984-5$ & 1.46 & 2.62 & 13 \\
$1985-6$ & 1.07 & 2.00 & 13 \\
$1986-7$ & 1.92 & 2.54 & 13 \\
$1987-8$ & 0.91 & 0.91 & 11
\end{tabular}




\subsubsection{Long-tailed cuckoos}

See Chapter 7 for an account of long-tailed cuckoo predation and nest parasitism.

\subsubsection{Mortality}

Mortality was measured only at Knobs Flat. For the purposes of estimating mortality rates, yellowheads were divided into the following easily recognisable classes: juveniles, immatures, and adults. Birds were also classified by sex. Age classes and sexes are described in Chapters 3 and 4.

\subsubsection{Summer}

Summer mortality was monitored by regularly checking for the presence of known birds within the study area. During the summer, birds were territorial and the disappearance of any bird before it finished breeding was regarded as indicating its death.

During the first three years of this study I detected no mortality of adult or immature yellowheads in summer, but during the 1987-8 breeding season 6 (50\%) of the 12 females that nested in or near my study area were preyed upon by stoats (see section 5.3.2.11).

\subsubsection{Winter}

Since my study area at Knobs Flat was part of a large forest block, birds moved freely in and out of it, particularly in the winter when they were not holding territories. Thus the disappearance of a bird between breeding seasons did not necessarily indicate its death.

Mortality was estimated by regarding the birds in my study area at the beginning of each breeding season as a sample of the population in the surrounding forest. Mortality rates were estimated from the age structure of samples from successive years.

Birds were regarded as being resident in the study area if they nested within it, or for non-breeding birds, if they spent most of their time in it. The number of juveniles in the study area was estimated by multiplying the average number of fledglings produced by all the pairs that were monitored, by the number of pairs that attempted to breed within the study area.

Table 5.16 shows the numbers of birds in each class at the end of each breeding season, and the winter mortality rates. The five females that were killed during the breeding 
season of 1987-88 within the study area are not included in the winter mortality rate, and all chicks that died are assumed to have died during the winter, though some may have died during the summer.

\begin{tabular}{|c|c|c|c|c|c|c|c|c|}
\hline \multirow[b]{4}{*}{$\underline{\text { Year }}$} & \multicolumn{5}{|c|}{ Table 5.16} & \multirow{2}{*}{\multicolumn{3}{|c|}{$\begin{array}{l}\text { "Juv-Imm" is the } \\
\text { r survival rates of }\end{array}$}} \\
\hline & $\begin{array}{l}\mathrm{n} \mathrm{st} \\
\mathrm{rsu}\end{array}$ & rat & $\begin{array}{l}\text { iter } \\
\text { iven } \\
\text { imn }\end{array}$ & es a & $\begin{array}{l}\mathrm{s} \text { at } \mathrm{r} \\
\text { is th } \\
\text { dults }\end{array}$ & & & \\
\hline & \multicolumn{2}{|c|}{ Adults } & \multicolumn{3}{|c|}{ Immatures } & \multirow{2}{*}{$\begin{array}{l}\text { Juveniles } \\
\text { (estimated) }\end{array}$} & \multirow{2}{*}{\multicolumn{2}{|c|}{$\begin{array}{c}\text { Over-winter } \\
\text { Survival } \\
\text { Juv-Imm Imm+ }\end{array}$}} \\
\hline & M & $\mathrm{F}$ & $\mathrm{M}$ & $\mathrm{F}$ & Unk & & & \\
\hline $1984-5$ & 12 & 12 & 3 & 0 & 1 & 31.44 & - & - \\
\hline $1985-6$ & 16 & 13 & 4 & 1 & 7 & 26.00 & 0.38 & 1 \\
\hline 1986-7 & 18 & 12 & 3 & 0 & 1 & 30.48 & 0.15 & 0.73 \\
\hline $1987-8$ & 16 & 14 & 0 & 0 & 1 & 10.00 & 0.03 & 0.8 \\
\hline 1988-9 & 11 & 7 & 1 & 0 & 4 & - & 0.50 & 0.72 \\
\hline $\begin{array}{l}\text { Weighted } \\
\text { average }\end{array}$ & & & & & & & 0.22 & 0.83 \\
\hline
\end{tabular}

\subsubsection{Incubation and nestling periods}

Incubation and nestling periods of 21 and 18 days respectively have previously been recorded by Soper (1960) at one yellowhead nest in the Eglinton Valley. While Soper's incubation period is within the range I recorded (19 - 22 days), his 18 day nestling period is much shorter than I recorded (21 - 22 days) and suggests that the chicks in his nest fledged early.

Yellowhead incubation and nestling periods are longer than those of the whitehead, and most other New Zealand forest passerines (see Gill 1983). This is consistent with Lack's (1948) observation that passerines which nest in holes or have enclosed nests, tend to have longer incubation and nestling periods than those with open nests.

\subsubsection{Breeding season}

Accounts of yellowhead breeding prior to this study record breeding in only November and December (Guthrie-Smith 1936, Soper 1963, Read 1987), though Read (1987) notes 
that a few nests have been recorded from mid-October till early March. This study confirms that the yellowhead breeding season can last for up to $4^{1} / 2$ months from mid October until early March.

Given the incubation (20 days) and nestling (221/ 2 days) periods of the species, and allowing 5 days to lay a clutch it would be possible for yellowheads to raise three broods in a breeding season. However most pairs raise only two.

The breeding season of yellowheads is similar in length to that of other forest insectivores which raise two broods (e.g. grey warblers, fantails (Gill et al. 1983), and brown creepers (Cunningham 1985) at Kowhai Bush), but shorter than that of robins which raised three clutches at Kowhai Bush (Gill et al. 1983).

Yellowheads at Knobs Flat start breeding later than the four well-studied insectivorous forest birds at Kowhai Bush (Gill et al. 1983 and Cunningham 1985). Furthermore, the birds at Plato Creek start breeding even later, as do those in the Hawdon Valley in Arthur's Pass National Park (Read 1987).

Several authors have suggested that the timing of breeding is related to food supply (e.g., Perrins 1970, Davies \& Lundberg 1985, and Gill et al. 1983), and Read (1987) suggested that the late, short breeding season, and lack of second clutches of yellowheads in the Hawdon Valley was related to a shortage of food. He cited the low density of yellowheads in the Hawdon as corroborating evidence for food shortage. The same explanation may apply at Plato Creek.

It is possible that the late, short breeding seasons and lack of second clutches at the Hawdon and Plato Creek, result not from a year-round shortage of food, but rather a delayed increase in the normal summer-time increase in food supply which is related to the climate and altitude of these two places. Both the Hawdon Valley and Plato Creek are at higher altitudes and have colder climates than Knobs Flat.

A late start will shorten the breeding season and limit the yellowheads to raising one brood. At Knobs Flat, most yellowheads can raise two broods and moult before winter, but at higher altitudes they may not be able to raise more than one.

It is probably not possible to determine whether the late, short breeding seasons, and lack of second clutches at Hawdon Valley and Plato Creek result from a general shortage of food at these places, or simply a later summer-time increase in the food supply. 
Yellowheads, like many other New Zealand passerines, breed slowly and have small clutches. Gill (1982), following an hypothesis developed for tropical birds, argued that long breeding seasons and small clutches have evolved in New Zealand birds in reponse to an equable climate. In the tropics, and perhaps New Zealand, food supply is virtually constant and populations remain close to the limit set by food. Extra food is always hard to obtain so birds raise small families slowly. This contrasts with the northern temperate zone where numbers of sedentary insectivorous song birds are reduced by a severe shortage of food in late winter. With the flush of invertebrates coinciding with the breeding season in sping, surviving sedentary birds and returning migrants raise large families.

Small clutch size is but one of a suite of adaptations that characterise temperate Australian passerines. Thomas (1974) suggests that the breeding stategies of temperate Australian land birds may be responses to a food supply that is "never abundant", and Gill (1982) noted the similarity of Thomas's and his own interpretations. Russell (1989) has recently shown that, in light of new phylogenies constructed by Sibley and Ahlquist (1985), the high incidence of co-operative breeding amongst Australian birds may result from shared ancestry, not from shared ecological conditions. The same argument may well apply to the small clutch sizes and slow breeding of New Zealand birds.

\subsubsection{Variation in stoat numbers}

During most years stoats are at low densities in the Eglinton Valley, but in the summer of 1987-8 the number of stoats and the predation rate of yellowhead nests were much higher. The relationship between beech mast (heavy seeding), mouse and stoat number has been well documented by King (1983) who did some of her work in the Eglinton Valley. King found that beech forests had irregular heavy seedfalls (masts) and that during the following winter, spring and summer mice numbers rose, as did stoat numbers during the following summer. King found that in the summers following beech mast, more birds were eaten by stoats than in other summers.

The rise in stoat numbers I observed followed exactly this pattern. In the spring of 1986 there was a heavy flowering of beech in the Eglinton Valley. In the following autumn there was a heavy seedfall, and in the summer of 1987-8 stoat numbers rose dramatically. 


\subsubsection{Stoat predation}

Stoats take higher numbers of yellowhead nests with nestlings than nests with eggs. A simple explanation is that nests with nestlings are more conspicuous because adult birds visit nests frequently to feed nestlings. Alternatively, because stoat numbers rise during the yellowhead's breeding season, there may be fewer stoats and less predation when yellowheads have eggs, but more by the time the eggs have hatched.

Stoat plagues have a dramatic effect on yellowhead breeding and mortality probably because yellowheads are hole-nesters and only the females incubate. Stoats do most damage, not by eating eggs and nestlings, but by eating incubating yellowheads. Birds that have open nests may lose eggs and nestlings to stoats, but the incubating birds will often detect a stoats approach, escape and renest. Such species will respond to high levels of stoat predation by an increased number of nesting attempts. Yellowheads cannot detect a stoats approach until the stoat puts its head into the nest hole, by which time its too late, and when the female as well as the brood is eaten there is no prospect of renesting.

If both male and female yellowheads incubated, instead of just females, the total number of birds taken by stoats would be the same, but half would be males and half females. By the next breeding the surviving birds could have formed new pairs and may breed again. If only females are taken there is little prospect of the surviving males forming new pairs because there is a shortage of females.

The effect of stoat plagues on yellowheads is probably worse than on other forest birds, not only because they are hole nesters, but because they breed relatively late. Many small forest insectivores will have already raised one clutch, or even finished breeding entirely before stoats born in October become independent in December. In contrast the peak breeding activity of yellowheads coincides with the increase in the stoat population as young stoats become independent (King and Moody 1982a and 1982b).

\subsubsection{Productivity, mortality and stoat plagues}

Table 5.17 compares the breeding success of yellowheads at Knobs Flat during normal and stoat plague years with the breeding of other New Zealand forest dwelling, insectivorous passerines. The productivity from only the first clutch during non-stoat plague years is given as an indication of the likely productivity of a population that raised only one clutch of chicks a year. 
Table 5.17

Breeding success of New Zealand forest-dwelling insectivorous passerines

\begin{tabular}{|c|c|c|c|c|c|c|c|}
\hline Species & $\begin{array}{c}\% \\
\text { nests } \\
\text { fledge }\end{array}$ & $\begin{array}{c}\% \\
\text { eggs } \\
\text { hatch }\end{array}$ & $\begin{array}{c}\% \\
\text { nestlings } \\
\text { fledge }\end{array}$ & $\begin{array}{c}\% \\
\text { eggs } \\
\text { fledge }\end{array}$ & $\begin{array}{l}\text { No. } \\
\text { fledge } \\
\text { /pair }\end{array}$ & Where & Reference \\
\hline Grey warbler & 42 & 70 & 54 & 38 & 4.0 & Kaikoura & Gill 1982 \\
\hline Fantail & - & 83 & 55 & 46 & - & Kaikoura & Powlesland 1982 \\
\hline Fantail & 57 & - & - & - & 2.5 & Tiritiri I. & McLean 1984 \\
\hline Fantail & - & - & - & - & 2.6 & Cuvier I. & $\begin{array}{l}\text { McLean \& } \\
\text { Jenkins } 1980\end{array}$ \\
\hline Robin & 32 & - & - & - & 3 & Kaikoura & Flack 1976 \\
\hline Robin & - & 63 & 42 & 26 & $2.1-2.9$ & Kaikoura & Powlesland 1983 \\
\hline Rifleman* & 50 & 58 & 79 & 45 & 3 & Dunedin & Gray 1969 \\
\hline Rifleman & - & - & 58 & - & - & Wellington & Gaze 1978 \\
\hline $\begin{array}{l}\text { Brown } \\
\quad \text { creeper }\end{array}$ & - & 64 & 57 & 36 & 3.2 & Kaikoura & Cunningham 1985 \\
\hline Whitehead & 43 & - & - & - & 1.1 & $\begin{array}{l}\text { Little } \\
\text { Barrier I. }\end{array}$ & $\begin{array}{l}\text { McLean \& } \\
\quad \text { Gill } 1988\end{array}$ \\
\hline $\begin{array}{l}\text { Yellowhead } \\
\text { normal yr } \\
\text { stoat yr } \\
\text { one clutch }\end{array}$ & $\begin{array}{l}58 \\
40 \\
-\end{array}$ & $\begin{array}{l}50 \\
69 \\
-\end{array}$ & $\begin{array}{l}76 \\
38 \\
-\end{array}$ & $\begin{array}{l}33 \\
23 \\
-\end{array}$ & $\begin{array}{l}2.4 \\
0.9 \\
1.5\end{array}$ & $\begin{array}{l}\text { this study } \\
\text { this study } \\
\text { estimated fr }\end{array}$ & m this study \\
\hline
\end{tabular}

During normal years yellowhead breeding success was within the range recorded for comparable New Zealand birds. During stoat plagues, however, their productivity was lower than comparable species. Yellowhead populations that produced only one clutch of chicks a year had a low productivity compared with other forest-dwelling passerines.

Table 5.18 compares the survival rates of yellowheads at Knobs Flat during normal years with that of other New Zealand forest dwelling, insectivorous passerines.

\section{Table 5.18}

Survival rates of New Zealand forest-dwelling insectivorous passerines

\begin{tabular}{l|ccll} 
Species & $\begin{array}{c}\text { Fledging } \\
\text { to breeding } \\
\text { survival }\end{array}$ & $\begin{array}{c}\text { Adult } \\
\text { survival }\end{array}$ & Place & Reference \\
Rifleman & 0.32 & 0.59 & Kaikoura & Sherley 1985 \\
Grey warbler & 0.045 & 0.815 & Kaikoura & Gill 1982 \\
Robin & 0.169 & 0.386 & Kaikoura & Powlesland 1983 \\
Brown creeper & 0.105 & 0.824 & Kaikoura & Cunningham 1985 \\
Yellowhead & 0.185 & 0.826 & This study &
\end{tabular}


Despite taking two years to reach breeding age, yellowhead recruitment is higher than other comparable New Zealand birds.

The real question about productivity and mortality of yellowheads is not whether they are higher or lower than other species, but whether productivity is sufficient to balance mortality. This question is investigated in Chapter 6.

\subsubsection{Other predators}

Stoats are the only common mammalian predator in the Eglinton Valley. Of the three species of rat in New Zealand only ship rats are known from the Eglinton, and they are rare (King 1983 and pers. obs.). Cats are also rare and some are recently released pets one shot in the valley recently was a castrated male. Ferrets (Mustela furo) and weasels (M. nivalis) are not known from the Eglinton and must be rare or absent.

My conclusion that most of the nest predation I recorded was by stoats (section 5.3.2.11), does not mean that yellowheads are not vulnerable to predation by other species. Ships rats, in particular, are known to be competent arboreal predators (Moors 1983) and in areas where they are common they are likely to have a significant impact on yellowhead populations.

\subsubsection{Density dependence}

This study reveals some evidence for density dependent productivity and juvenile mortality. Behavioural observations (Chapter 3) and the fact that not all of the pairs within my study area attempted to breed suggests that territorial behaviour may limit the density of breeding pairs and thus productivity. Evidence for density dependent juvenile mortality comes from the fact that the juvenile survival was highest in the year following a stoat plague. This is a time when one might expect high juvenile mortality because of high stoat numbers, instead there was low mortality perhaps in response to the low numbers of yellowheads present. O'Donnell (pers. comm.) working on yellowheads in the Hawdon Valley has recorded juvenile survival of about 67\%, and this too could be related to the low density and chick production there.

The effects of density dependent juvenile survival are explored more fully in the next chapter. 


\subsubsection{Predation of fledglings}

Read (1987) observed that yellowhead fledglings are fed on, or close to, the ground for the first few hours out of the nest, and he suggested that this might make the species particularly vulnerable to mammalian predation especially during a beech seeding year when predator numbers are high. I also observed fledgling yellowheads on the ground, but I observed many more that climbed straight into the canopy when they left the nest. Furthermore, survival of yellowheads from fledgling to year one was higher in the year when stoat numbers were high, than it was in the other years. It seems unlikely that predation of fledglings on the ground has a significant effect on yellowhead population dynamics.

\section{5}

\section{CONCLUSIONS}

1. Yellowheads can raise two broods per year, though they do not always do so.

2. Yellowheads breed from early October until early March at Knobs Flat.

3. Eggs are laid at one or two day intervals.

4. The incubation period is about 20 days.

5. The nestling period is about $22^{1} / 2$ days.

6. Clutch sizes vary from 1 to 4 , most are 3 .

7. During most years there is no significant nest predation by introduced mammals.

8. During a year when stoat numbers were high, $67 \%$ of nests were preyed upon by stoats.

9. During years when stoat numbers are low yellowhead productivity and mortality is well within the range recorded for other forest-dwelling passerines.

10. The productivity of yellowheads that produce only one brood a year is lower than that of most other forest-dwelling passerines.

11. During years when stoat numbers are high yellowhead productivity and mortality is lower than for most other forest-dwelling passerines. 
12. There is some evidence that there are density dependent mechanisms influencing the productivity and survival of chicks.

13. Predation of fledglings is unlikely to significantly affect yellowhead population dynamics. 


\section{CHAPTER 6}

\section{POPULATION MODELLING OF YELLOWHEADS}

6.1

INTRODUCTION

In the preceding chapter a four year study of productivity and survival of yellowheads at Knobs Flat, was described. During most years yellowhead survival and productivity were comparable with that of other small New Zealand forest-dwelling insectivorous passerines. However, during stoat plagues, which occurred after beech mast, productivity and female survival was much reduced. Furthermore, productivity and juvenile survival seemed to be controlled by density-dependent mechanisms and some populations raised only one brood a year whereas others raised two. I suggested that this might be due either to reduced length of breeding season at high altitudes or to reduced productivity of some areas.

Recent dramatic declines in yellowhead numbers in Arthur's Pass and Fiordland National Parks following stoat plagues prompted the development of a model of the likely long-term effects of stoat plagues on yellowhead survival (Elliott \& O'Donnell 1988). Elliott and O'Donnell predicted that yellowhead populations that raised only one brood a year are unlikely to be able to survive repeated stoat plagues. In this chapter another years figures were included and an attempt made to model in greater detail the likely effects of stoat plagues, multiple broods, density dependent mechanisms and population size on yellowheads.

A key aim of this modelling study was to assess whether periodic stoat plagues could alone account for the decline of yellowheads. In particular I aimed to construct a model of yellowhead populations that matched the population changes observed at Knobs Flat and at Arthur's Pass. The following questions were addressed using this model:

1. Does yellowhead productivity match mortality?

(i) during normal years.

(ii) during stoat plague years.

(iii) if yellowheads have only one brood a year.

2. Can yellowheads survive repeated stoat plagues?

(i) in areas where they have two broods a year.

(ii) in areas where they have only one brood. 
3. What effect does population size have on the yellowhead's ability to withstand stoat plagues?

In attempting to answer these questions particular attention was paid to the survival of yellowhead populations

(1) after 100 years: the length of time that yellowheads have been suffering stoat predation in New Zealand.

(2) that suffer stoat plagues every 5 years. Stoat plagues occur following beech mast (King 1983), and these occur every 4 to 6 years (Wardle 1984).

The evidence for density dependent control of productivity and survival was equivocal. Consequently models were developed with and without density dependent mechanisms to determine which best matched observed population changes.

In general, two approaches can be taken to developing population models: deterministic and stochastic.

Classical (deterministic) demographic theory (Lotka 1907, Leslie 1945) as used by Elliott and O'Donnell (1988), assumes populations live in fixed environments and have fixed vital rates (fertility and mortality), but recently there has been considerable development of a more realistic stochastic theory that incorporates random temporal changes in vital rates (see review by Tuljapurkar 1989).

Deterministic models are more mathematically tractable than stochastic ones, but for populations with significant variation in vital rates, and for populations near extinction, they produce biased estimates of growth rates and extinction probabilities (Tuljapurkar 1989). Tuljapurkar and Orzack (1980) showed that " . . the most probable effect of unpredictable temporal fluctuation can be to drive populations into long-term decline in numbers."

In this study I initially developed a deterministic model that approximately matched observed population changes. The deterministic model was then used to assess the qualitative effects of changes in numbers of clutches and stoat plague frequency. Stochasticity was then added to the model to assess the extinction probabilities of yellowhead populations. 


\subsubsection{Population parameters}

Table 6.1 shows the population parameters of yellowheads at Knobs Flat during the 4 years of my study.

\section{Table 6.1}

Population parameters of yellowheads at Knobs Flat between 1984 and 1989

\begin{tabular}{l||cc|cccc} 
& \multicolumn{2}{|c|}{$\begin{array}{c}\text { Productivity } \\
\text { (Fledglings/pair) }\end{array}$} & \multicolumn{4}{c}{ Survival } \\
Year & 1st clutch & Total & fledglings & foles year & winter & summer \\
\hline & & & & - & - & 1 \\
$1984-5$ & 1.46 & 2.62 & - & 1.03 & 1.03 & 1 \\
$1985-6$ & 1.07 & 2.00 & 0.38 & 0.73 & 0.73 & 1 \\
$1986-7$ & 1.92 & 2.54 & 0.15 & 0.73 & 0.5 \\
$1987-8^{*}$ & 0.91 & 0.91 & 0.03 & 0.88 & 0.88 & - \\
$1988-9$ & - & - & 0.50 & 0.69 & 0.69 & -
\end{tabular}

* A stoat plague occurred this year

Elsewhere (Chapter 5) I have shown that fledgling production and female summer survival are much reduced in years when stoat plagues occur, and for this reason I distinguished between "stoat plague years" and "normal years" in all of the models that I developed. For the purposes of modelling, population parameters during stoat plague years were set at the values recorded during the 1987-8 breeding season at Knobs Flat. Non-plague year parameters were taken to be the means of the parameters during the other years of study at Knobs flat.

To compare the behaviour of populations that raise one brood a year with those that raise two, I assumed that the productivity of one-brood populations was the same as that of two-brood ones during their first brood. I have assumed that survival was the same, except that for one-brood populations during stoat plagues I set female summer survival at 0.67 which was the survival of females at Knobs Flat during their first clutch.

Density dependent productivity was simulated by setting a maximum number of breeding pairs. A population might rise above this level, but not all of the adult birds would breed. The Knobs Flat population appeared to behave in this way (see Chapter $3)$.

Density dependent fledgling survival was simulated by assuming that fledgling survival was related to the number of breeding pairs of yellowheads present (see Figure 6.1). 
Maximum survival (0.5) was taken from the fledgling survival following the 1987-8 breeding season when the yellowhead population at Knobs flat was at its lowest. Minimum fledgling survival (0.2) was taken from the average of all the other years, when the yellowhead population density was high.

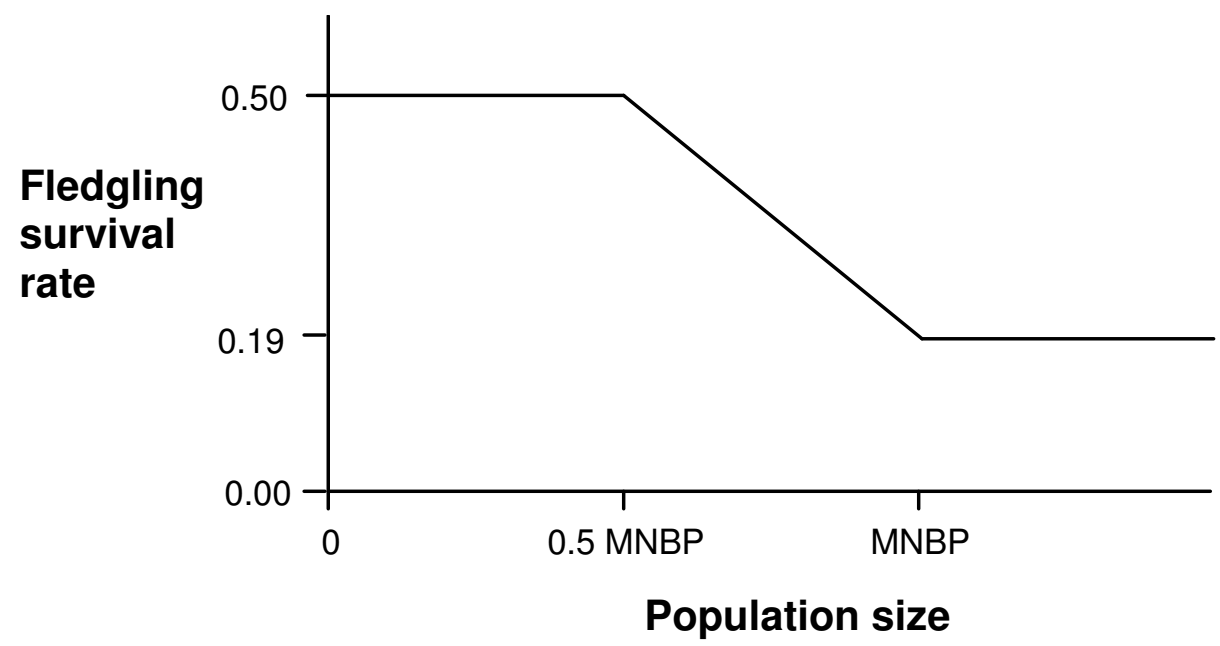

Figure 6.1: Fledgling survival rates used in density dependent yellowhead population simulation. $\mathrm{MNBP}=$ maximum number of breeding pairs; populations greater than this size will contain non-breeding adults.

Since the form of this relationship was chosen arbitrarily, the effect of changing the form was assessed by varying the population sizes at which production was maximal and minimal.

For comparison, a non-density dependent fledgling survival was calculated from the average fledgling survival over all years.

\subsubsection{Deterministic model}

For deterministic models the vital rates (productivity and mortality) were taken to be the weighted averages of the yearly rates. Implicit in calculating weighted averages was the idea that variation between years was due only to sampling variation and that yellowhead populations had constant parametric vital rates.

Simple deterministic models of populations with no density dependent controls are easily constructed using matrix algebra (Leslie 1945). For modelling yellowhead populations the productivity and survival of yellowheads in three age classes was incorporated in a stage-based population projection matrix (Lefkovitch 1965) of the form: 


$$
\left[\begin{array}{ccc}
0 & 0 & \mathrm{a}_{13} \\
\mathrm{a}_{21} & 0 & 0 \\
0 & \mathrm{a}_{32} & \mathrm{a}_{33}
\end{array}\right]
$$

where $\quad a_{13}=$ the number of female fledglings produced per year per breeding female

$\mathrm{a}_{21}=$ proportion of female fledglings that survive to become immatures

$\mathrm{a}_{32}=$ the proportion of immature females that survive to become adults

$\mathrm{a}_{33}=$ the proportion of adults that survive each year

Population projection matrices can be used to predict the population in one years time $(t+1)$ by multiplying them with vectors of population structure at the present time $(t)$ :

$$
\left[\begin{array}{l}
\mathrm{n}_{1} \\
\mathrm{n}_{2} \\
\mathrm{n}_{3}
\end{array}\right]_{\mathrm{t}+1}=\left[\begin{array}{ccc}
0 & 0 & \mathrm{a}_{13} \\
\mathrm{a}_{21} & 0 & 0 \\
0 & \mathrm{a}_{32} & \mathrm{a}_{33}
\end{array}\right]\left[\begin{array}{l}
\mathrm{n}_{1} \\
\mathrm{n}_{2} \\
\mathrm{n}_{3}
\end{array}\right]_{\mathrm{t}}
$$

where $\quad \mathrm{n}_{1}=$ the number of fledgling females.

$\mathrm{n}_{2}=$ the number of immature females.

$\mathrm{n}_{3}=$ the number of adult females.

Long-term population trends can be modeled by repeated multiplication, but there is an easier way; the dominant eigenvalues of population projection matrices are equal to population growth rates $(\lambda)$ (Groenendael et al. 1988) and are easily calculated. Growth rates are related to intrinsic rates of increase $(r)$ by:

$$
r=\log (\lambda)
$$

Growth rates greater than one indicate that productivity is greater than mortality and the population will increase. Rates less than one indicate a declining population.

To determine the frequency of stoat plagues that yellowhead populations can survive, population projection matrices for plague years were successively pre-multiplied by matrices for non-plague years and growth rates calculated for the product matrices. For example, if $\mathbf{A}$ was the population projection matrix for non-plague years and $\mathbf{B}$ the matrix for plague years, then $\mathbf{C}$, the matrix for one plague every n years, is given by:

$$
\mathbf{C}=\mathbf{A}^{\mathrm{n}-1} \mathbf{B}
$$

The dominant eigenvalue of $\mathbf{C}$ is not equal to the yearly growth rate $(\lambda)$, but to $\lambda^{\mathrm{n}}$. 


\subsubsection{Density dependence}

Incorporating density dependent effects into population projection matrices made them intractable and the effects of density dependence were investigated using computer simulation.

The simulations repeatedly calculated the number of fledglings, immatures and adults present each year from the product of the number present in the previous year and the appropriate survival or productivity estimate. Populations were seeded with 100 pairs, left for 100 years to reach stable age structures, then subjected to stoat plagues at fixed intervals. Population sizes were treated as continuous variables.

The simulation program was written in Basic and run on an Apple Macintosh computer (see Appendix A).

\subsubsection{Stochastic model}

For stochastic models vital rates were taken to be the un-weighted averages of the yearly rates and standard deviations were calculated. Implicit in unweighted averages is the notion that the variation in vital rates results from yearly variation in the parametric vital rates.

Stochasticity was introduced into the model by making each of the population parameters vary according to an appropriate probability distribution.

Adult survival was assumed to be normally distributed and was simulated using a random number generator that produced pseudo-random numbers with a normal distribution, mean of zero and standard deviation of 1. Linear transformation was used to convert these to random numbers with appropriate means and standard deviations. Survivals must always be bounded by 0 and 1 and following Boyce (1977) I rejected values that exceeded these bounds and generated new values to replace them.

During plague years adult female survival was assumed to be fixed and binomial sampling variation was the only source of variation. I wrote a simple algorithm to produce random numbers with binomial distributions to simulate this sort of variation.

Fledgling survival rates were assumed to vary according to the relationship shown in Figure 6.1, but binomial sampling variation was also added. 
Fledgling production was assumed to be normally distributed with means and standard deviations calculated from the four annual productivity estimates. Since there was only one estimate of the fledgling production during stoat plagues it was not possible to calculate a standard deviation for this parameter; I assumed it had the same standard deviation as fledgling production from first broods during non-stoat-plague years.

Nothing is known of the variability of intervals between stoat plagues in beech forests in New Zealand, though stoat plagues clearly do not occur at fixed intervals. The interval between stoat plagues was arbitrarily assumed to be normally distributed with a standard deviation equal to a sixth of the mean interval. That is, $99.74 \%$ of stoat plagues will occur within 0.5 and 1.5 times the mean interval.

As a further approach to reality population sizes were taken to be discrete variables and both males and females included in the simulation.

This simulation was initially undertaken by adding appropriate algorithms for variability to the density dependent program. The effect of stoat plague interval and initial population size was investigated by changing these parameters and running the simulation 100 times. This was found to take too long and the program was re-written and compiled in Fortran (see Appendix B).

\section{RESULTS}

\subsubsection{Deterministic model}

Table 6.2 shows the population projection matrices and growth rates during plague and non-plague years for populations of yellowheads that raise two broods a year and those that raise only one. The matrices were constructed assuming no density dependence. 


\section{Table 6.2}

Population projection matrices and growth rates of one and two brood yellowhead populations

\section{Normal year}

\section{TWO BROODS}

$$
\left[\begin{array}{ccc}
0 & 0 & 1.1933 \\
0.2246 & 0 & 0 \\
0 & 0.8295 & 0.8295
\end{array}\right]
$$

Growth rate $(\lambda)=1.0365$

Stoat-plague year

$$
\left[\begin{array}{ccc}
0 & 0 & 0.4545 \\
0.2246 & 0 & 0 \\
0 & 0.8295 & 0.4148
\end{array}\right]
$$

Growth rate $(\lambda)=0.6289$

Normal year

\section{ONE BROOD}

$$
\left[\begin{array}{ccc}
0 & 0 & 0.75 \\
0.2246 & 0 & 0 \\
0 & 0.8295 & 0.8295
\end{array}\right]
$$

$$
\text { Growth rate }(\lambda)=0.9761
$$

Stoat-plague year

$$
\left[\begin{array}{ccc}
0 & 0 & 0.4545 \\
0.2246 & 0 & 0 \\
0 & 0.8295 & 0.5558
\end{array}\right]
$$

Growth rate $(\lambda)=0.7194$

According to this model two-brood populations that never suffer stoat plagues would increase, whereas one-brood populations would slowly decline. Both types of population would decline if they suffered stoat plagues every year, but one-brood populations would decline more slowly than two-brood ones. This is because in twobrood populations during stoat plagues very few females manage to raise second broods, but large numbers of them die in the attempt.

\subsubsection{Frequency of stoat plagues}

Figure 6.2 shows the effect of the frequency of stoat plagues on growth rates of twobrood populations calculated by matrix algebra and assuming no density dependence. 


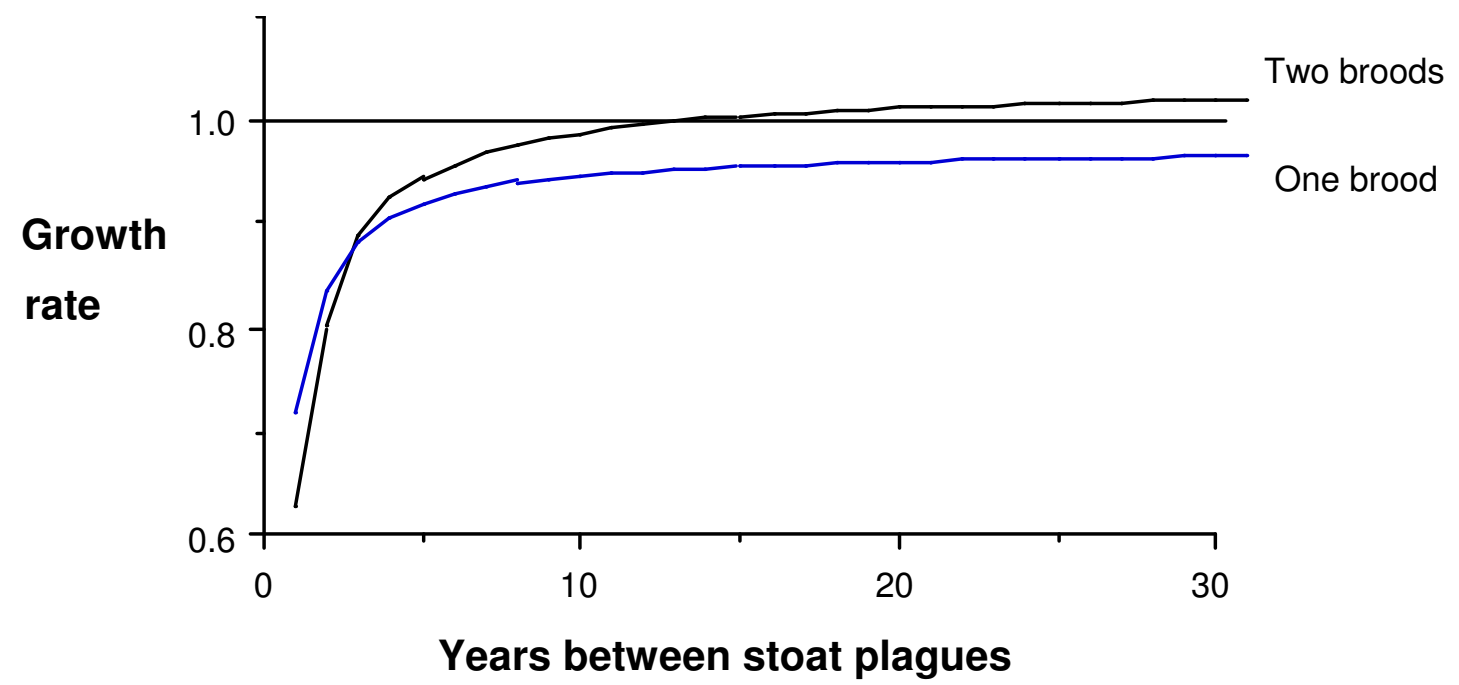

Figure 6.2: The effect of frequency of stoat plagues on the growth rate of yellowhead populations.

As the frequency of stoat plagues declines the population growth rates asymptotically approach the growth rates of populations suffering no plagues. Two-brood populations that suffer plagues less than once every 13 years will increase, whereas those that suffer plagues more frequently will decline. One-brood populations decline regardless of the frequency of stoat plagues since the growth rate never rises above 1 .

\subsubsection{Density dependence}

Figure 6.3 summarises effects of stoat plagues on one and two-brood populations with density dependent controls.

This simulation indicates that in the absence of stoat plagues populations with density dependent controls such as I have assumed, have the capacity to increase though they eventually stabilise. Two-brood populations stabilise with population sizes greater than the maximum number of breeding pairs (MNBP), i.e., they will have some nonbreeding adults in the population. One-brood populations stabilise with population sizes less than MNBP; all adults will breed. 
TWO-BROOD POPULATION

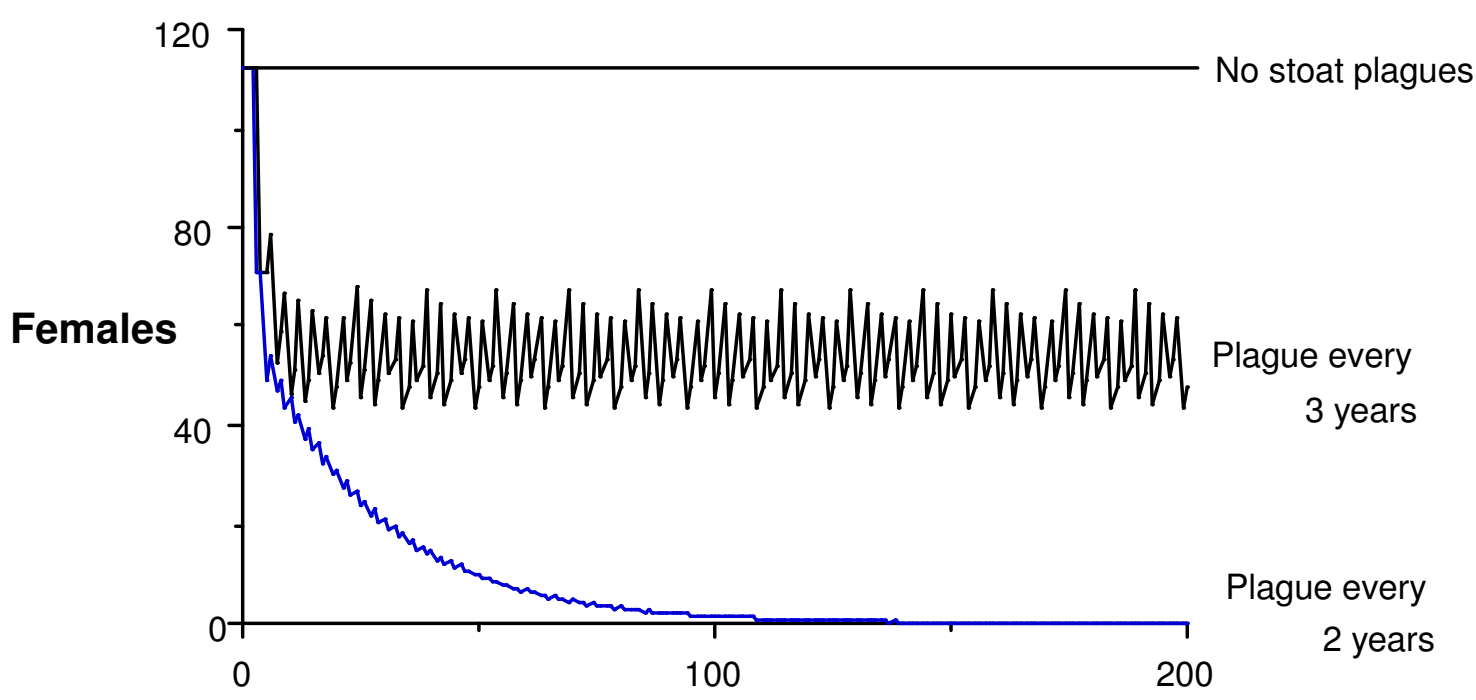

ONE BROOD POPULATION

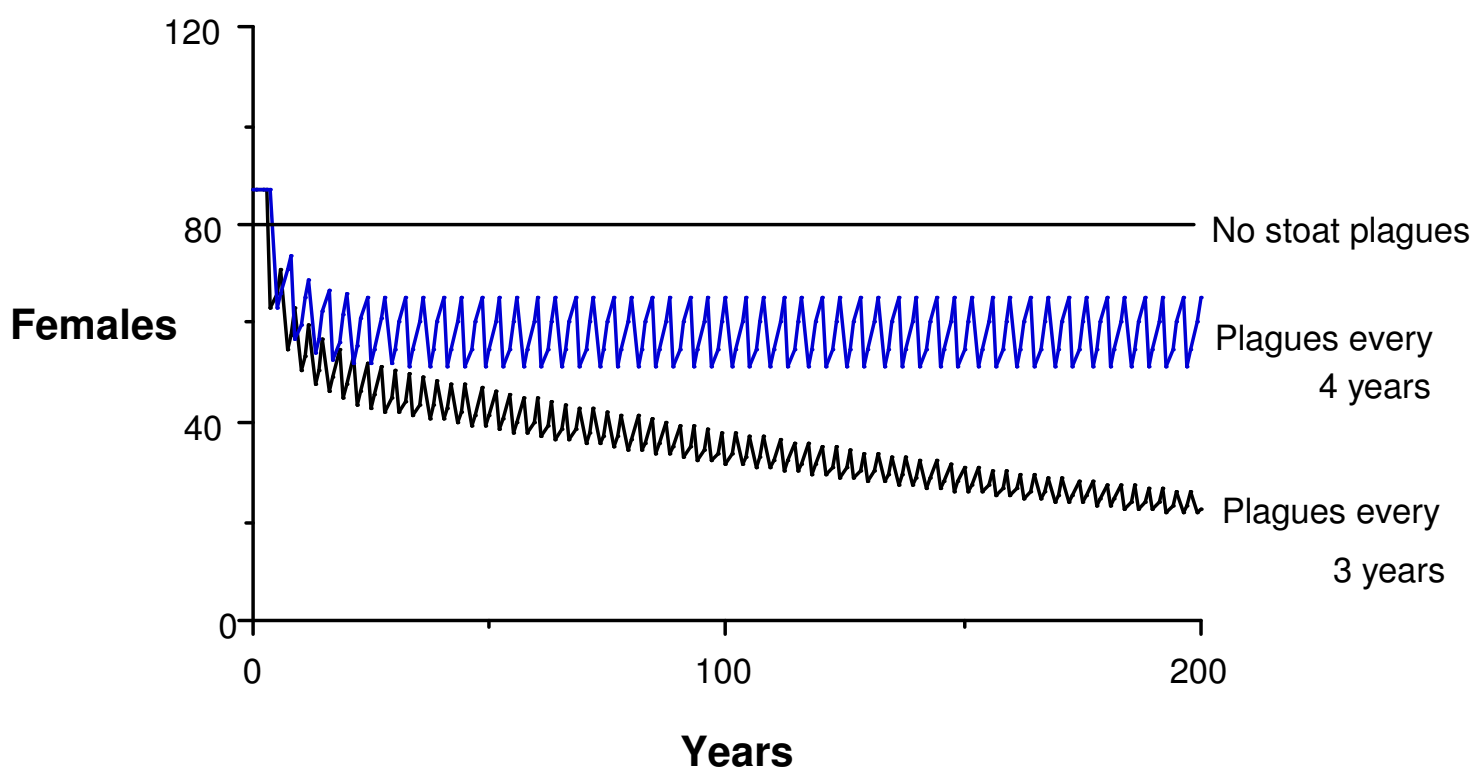

Figure 6.3: Density dependent population simulations for one and two-brood yellowhead populations.

Varying the form of the relationship between fledgling survival and population size had little effect on yellowhead populations. Provided the maximum survival $(0.5$ in this case) is sufficient to match mortality, a population will survive; the form of the relationship only effects the level at which the population stabilises.

One-brood populations are more susceptible to stoat plagues; they survive stoat plagues occurring no more frequently than once every four years, whereas two-brood populations survive them once every three years. 
Stoat plagues initially cause yellowhead populations to decline regardless of frequency, but at realistic plague frequencies, i.e., 4 - 6 years, populations quickly stabilise at low levels rather than decline to extinction. At Knobs Flat plagues occurring more than 8 years apart will enable the population to increase to carrying capacity. One-brood populations never increase to this level even if there are no plagues.

In both one and two brood populations stoat plagues cause an imbalance in the sex ratio. Even if stoat plagues occur infrequently the sex ratio is never even (Figure 6.4).

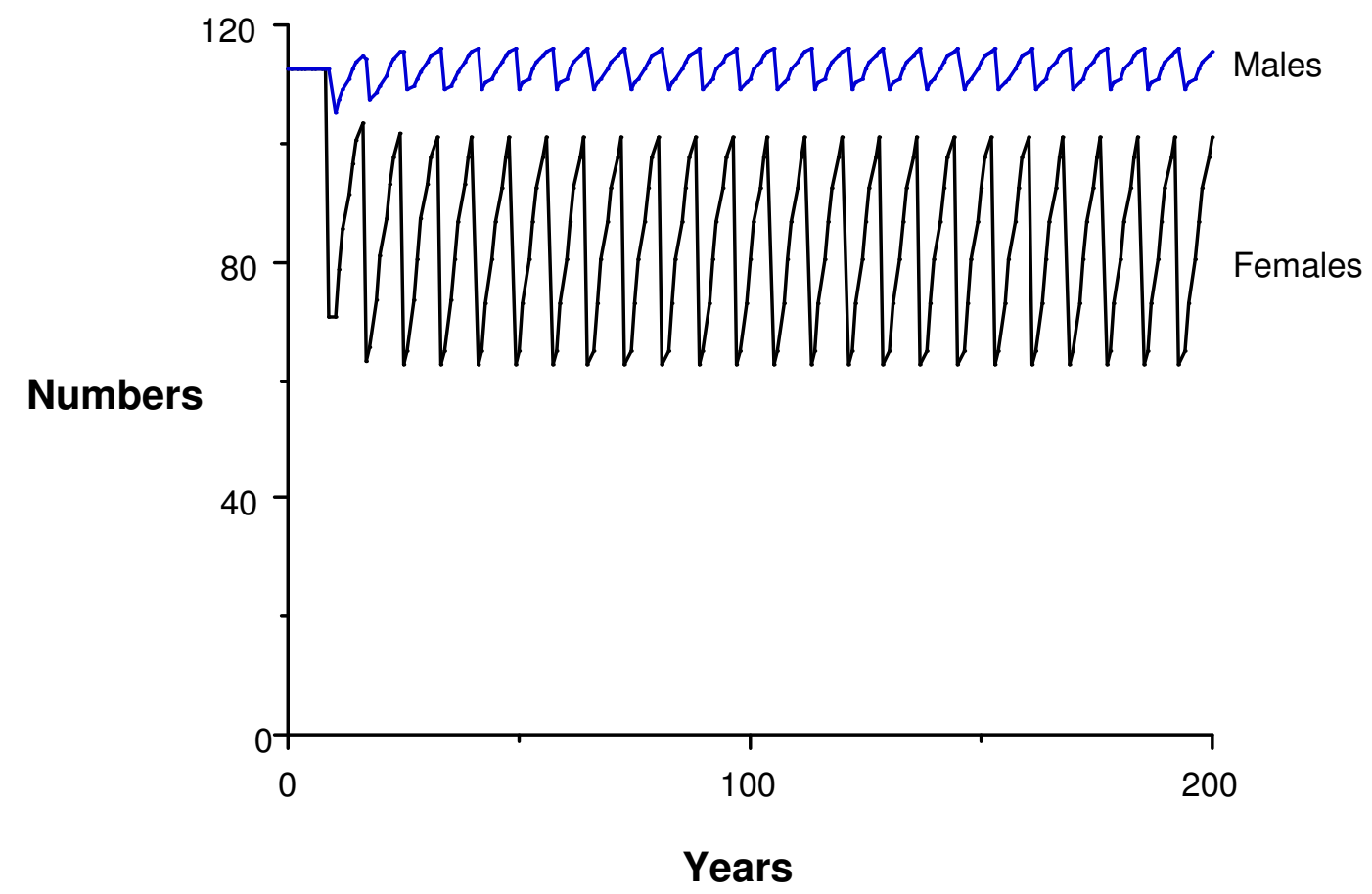

Figure 6.4: The effect of infrequent stoat plagues (1 every 8 years) on the sex ratio of a two-brood yellowhead population, such as at Knobs Flat.

\subsubsection{Stochastic model}

Stochastic population simulation gives rise to erratic population changes from which it is difficult to detect any long-term trends. However, by running 100 simulations and averaging their growth curves, trends emerged.

As predicted by Tuljapurkar and Orzack (1980) stochastic population models produced more pessimistic population projections than deterministic models.

With stoat plagues occurring every 5 years, all two-brood populations living in areas with carrying capacities greater than about 28 breeding pairs survive for 100 years 
(Figure 6.5), and their growth curves indicate they would survive indefinitely (Figure 6.6).

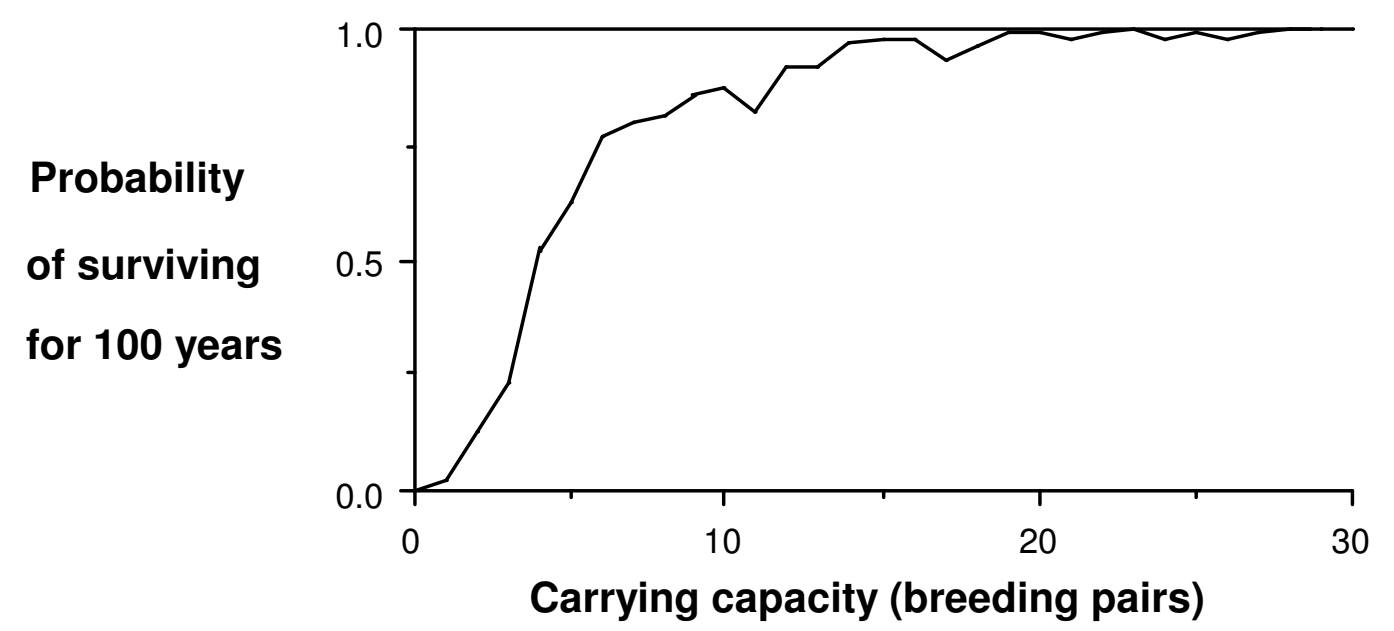

Figure 6.5: The relationship between probability of survival, and carrying capacity for two-brood populations suffering stoat plagues every 5 years. Note that the probability of survival of populations with carrying capacities greater than 28 pairs is 1 .

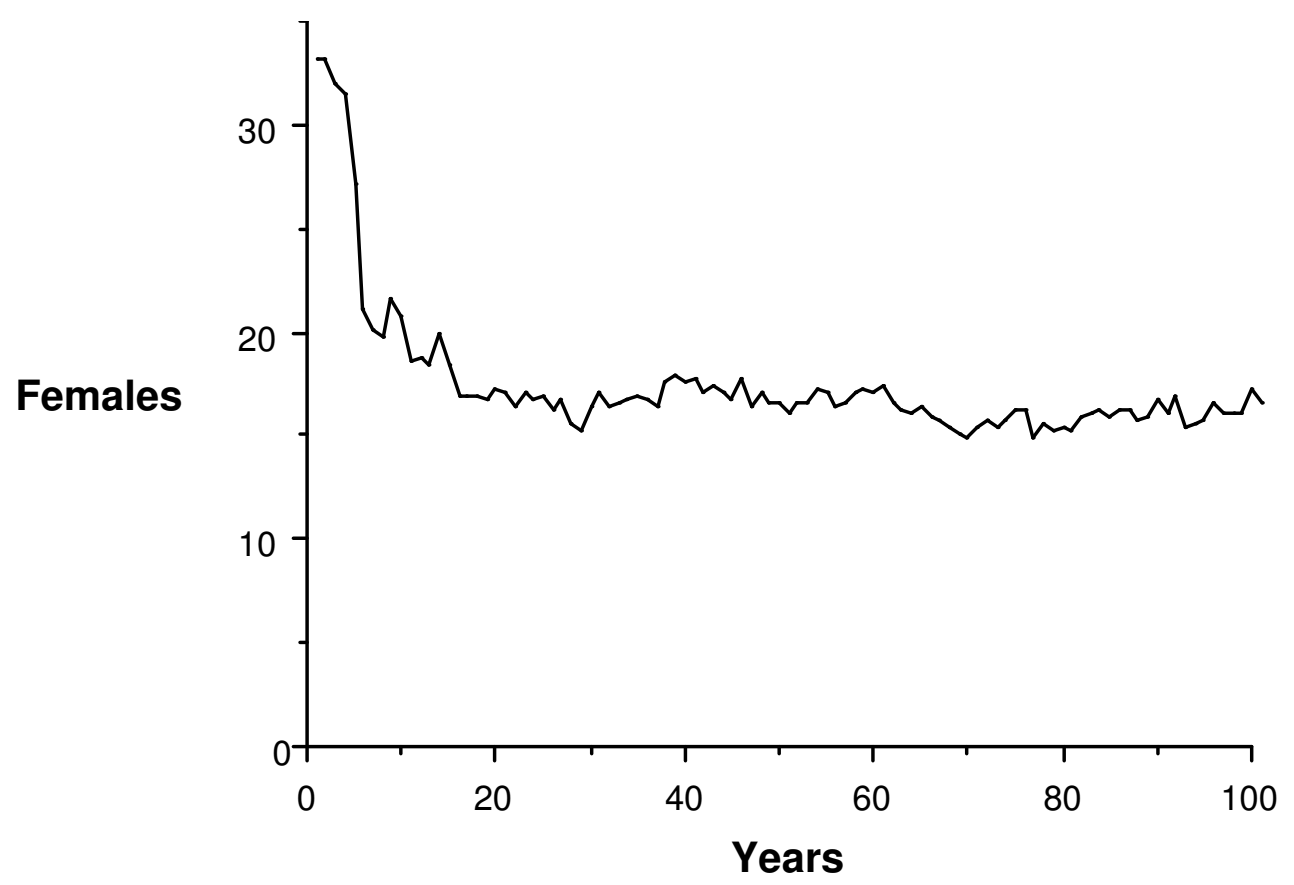

Figure 6.6: Growth curve for a two-brood yellowhead population with a carrying capacity of 30 pairs and suffering stoat plagues every five years. Note the population initially declines but eventually stabilizes.

In contrast one-brood populations suffering stoat plagues every 5 years declined regardless of the carrying capacity (e.g. Figure 6.7). Very large one-brood populations may still be extant after 100 years (Figure 6.8), but they have very low numbers and are heading for extinction. 


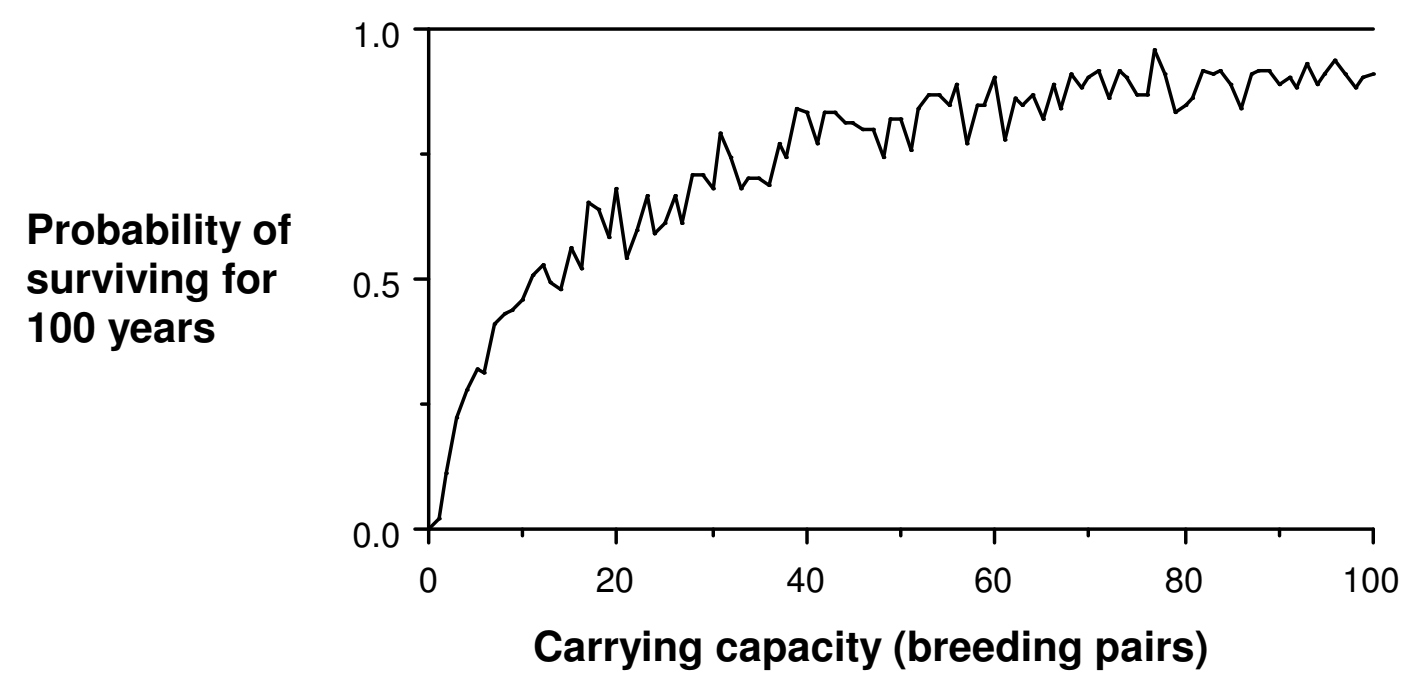

Figure 6.7: The relationship between probability of survival, and carrying capacity for one-brood populations suffering stoat plagues every 5 years. Note that the probability of surviving 100 years has not approached 1 even for large populations of greater than 100 pairs.

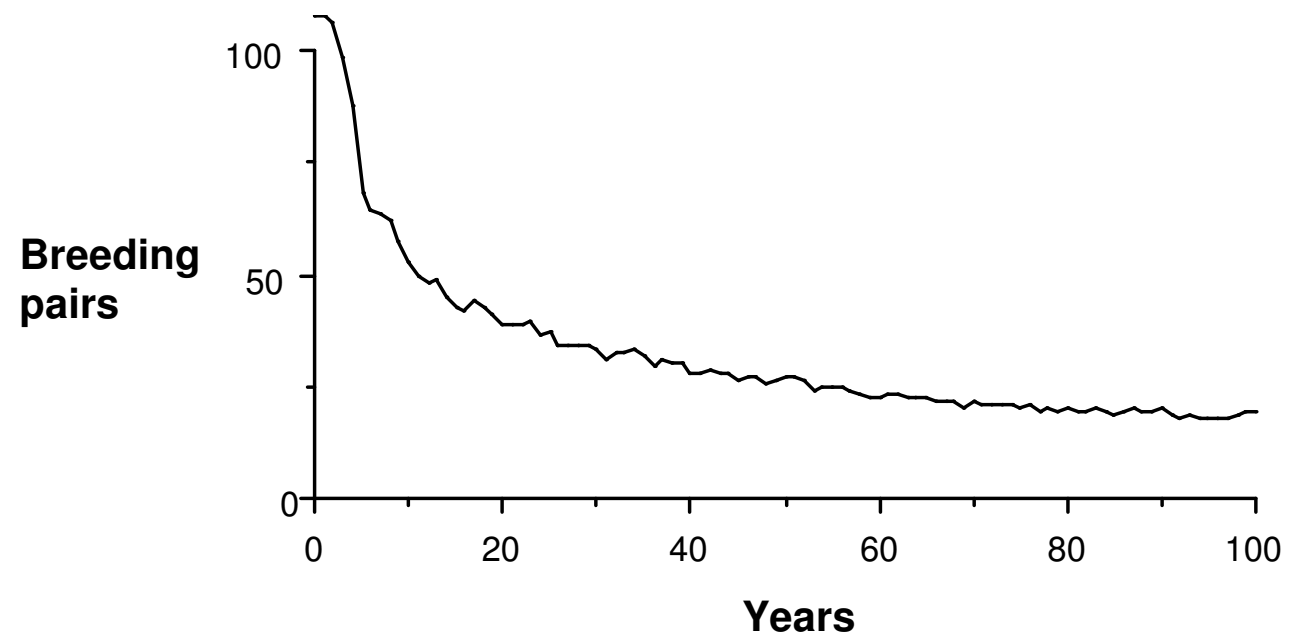

Figure 6.8: Growth curve for a one-brood yellowhead population with a carrying capacity of 100 pairs and suffering stoat plagues every five years. Note the population does not appear to stabilise.

6.4

DISCUSSION

The deterministic matrix model of yellowhead populations without density dependent controls does not closely match observations of yellowhead populations. 
Firstly, yellowhead populations clearly will not increase indefinitely in the absence of stoat plagues; there must be some regulatory mechanisms.

Secondly this model predicts that any population that suffers stoat plagues more than once every 13 years will decline. King (1983) recorded two stoat plagues with an interval of 4 years in the Eglinton Valley and Wardle (1984) noted that beech mast (which leads to stoat plagues) occurs on average every 4 to 6 years. It seems likely that the average time between stoat plagues is much less than 13 years in the Eglinton valley. Despite this the yellowhead population in my study area in the Eglinton Valley was apparently at carrying capacity during some of the breeding seasons before the stoat plague. It is clear that yellowhead populations can recover from stoat plagues in less than the 13 years predicted by this model predict.

In contrast deterministic simulations with density dependent mechanisms match observed population changes quite well. They indicate that two-brood populations suffering stoat plagues at realistic intervals will survive and even occasionally rise to carrying capacity as they do at Knobs Flat. They indicate that one brood populations suffering stoat plagues at realistic intervals will be at low density, as are the populations at Plato Creek and the Hawdon Valley.

The productivity of yellowheads during stoat plague years does not match mortality in both one and two-brood populations; yellowheads would not survive if predator numbers were constantly high rather than just periodically high. This could account for the early disappearance of yellowheads from the podocarp forests of central Westland and Stewart Island. Fruiting is much less periodic in these diverse forests than in low diversity beech forests and consequently rodent and stoat numbers are likely to be constantly high, rather than periodically high.

One-brood yellowhead populations are not as severely affected by stoat plagues as two brood ones, because females do not run the risk of being caught on the nest for as long as females in two brood populations. However, the productivity of one-brood populations is not as high as that of two-brood populations in non-stoat plague years, and they are less able to recover from stoat plagues.

If the density dependent mechanism that controls fledgling survival is anything like that in Figure 6.1, one-brood populations will rarely reach carrying capacity even when there are no stoat plagues. Repeated stoat plagues reduce both one and two-brood populations to below their carrying capacities, though sufficient gap between 
consecutive stoat plagues would enable two-brood populations to temporarily reach carrying capacity.

Repeated stoat plagues will also prevent recovery from the biased sex ratios caused by the female yellowhead's susceptibility to stoat predation. Sex ratios will remain biased between stoat plagues.

The stochastic population simulations indicate that all but the smallest yellowhead populations that raise two broods a year will survive repeated stoat plagues. Populations that raise only one brood a year will slowly decline if they suffer stoat plagues every 5 years, and only very large populations will be extant after 100 years.

If the yellowheads in places where they have declined or disappeared, raise or raised only one brood a year, then the occurrence of periodic stoat plagues will cause a slow decline, and this alone could explain much of the reduction in yellowhead distribution that has occurred since stoats arrived in New Zealand.

This study confirms (yet again) Tuljapurkar and Orzack's (1980) assertion that variation in vital rates reduces the growth rate, and increases the likelihood of extinction.

The overall picture that population modelling produces of yellowhead populations is as follows:

Yellowheads are vulnerable to predation, but provided their productivity is high, and the periods of predation infrequent, they survive. In areas where predator numbers are continuously high yellowheads are likely to disappear very quickly.

Yellowhead populations in favourable habitats that produce two broods a year, are able to survive repeated stoat plagues occurring about once every five years. Such populations may occasionally rise to above their carrying capacities and at such times there may be significant emigration. Populations in less favourable habitats that produce only one brood a year decline, so that after 100 years of stoat plagues most small populations are extinct and only a few once-large-but-now-small populations remain. One-brood populations never rise above their carrying capacities and there is unlikely to be emigration from them.

Over most of their range yellowheads now exist as a series of relatively isolated populations. Where some of these populations have two-broods it is possible that emigrating birds will occasionally recolonise areas from which one-brood populations 
have become extinct. Where all populations in an area are one-brood populations, recolonisation will never occur and all such populations will eventually become extinct. 


\section{CHAPTER 7}

\section{YELLOWHEADS AND LONG-TAILED CUCKOOS}

\section{1} INTRODUCTION

The yellowhead's hole nesting habits have been strongly implicated in its decline (see Chapters 5 and 6). Hole nesting is often regarded as an adaptation to reduce nest predation (Alerstam \& Högstedt 1981, von Haartman 1957, Lack 1968), and Soper (1976) suggested that in yellowheads hole nesting was a recently acquired adaptation to reduce nest parasitism and predation by long-tailed cuckoos. In Chapter 5 I showed that long-tailed cuckoos cause a significant proportion of yellowhead nest failures, and this would suggest that hole nesting is not an effective cuckoo-avoidance adaptation.

The long-tailed cuckoo breeds only in New Zealand, though it overwinters in a wide area of the tropical Pacific. It is a brood parasite, with the yellowhead, whitehead, and brown creeper being its main hosts. Its breeding habits and migration have attracted the interest of ornithologists, but these same habits along with its cryptic behaviour make it a difficult subject for study. Fulton (1904) exhaustively (perhaps exhaustingly) reviewed the information on the species at the time, and more recently it has caught the attention of Ian McLean (McLean 1982, 1985, 1987, 1988, McLean and Waas 1987). Despite these attentions some basic aspects of the birds biology have remained a mystery.

In this chapter the breeding and predatory habits of long-tailed cuckoos at Knobs Flat are described and their effect on yellowheads assessed. The main aim of this chapter was to assess Soper's (1976) assertion that hole nesting in yellowheads is an adaptation to reduce cuckoo parasitism.

7.2 METHODS

During a study of yellowhead breeding in 30 ha of beech forest at Knobs Flat between 1984 and 1988, ninety-five yellowhead nests were found (see Chapter 5). Each nest was described in detail and regularly monitored while in use. Special attention was paid to nests in which there was evidence of long-tailed cuckoo brood parasitism or predation. 
RESULTS

\subsubsection{Nest parasitism}

Six $(6.3 \%)$ of the 95 yellowhead nests found were parasitised by long-tailed cuckoos.

\subsubsection{Nest sites}

To determine whether nest hole size had any influence on whether or not a nest was likely to be parasitised by long-tailed cuckoos, the cross-sectional area of entrance holes, cavity depth and cavity cross-sectional area of cuckoo-parasitised nests were compared with unparasitised ones (Table 7.1). Mann-Whitney tests were used to test for significant differences. Cuckoo-parasitised nests had significantly larger entrance holes than unparasitised nests. Unparasitised nests were deeper than parasitised ones, but the difference was not significant.

\section{Table 7.1}

Comparison of yellowhead nests parasitised by long-tailed cuckoos with those not parasitised, and Mann-Whitney tests for difference.

\begin{tabular}{l|rrrc} 
& \multicolumn{4}{|c}{ Mean } \\
Variable & Cuckoo & No cuckoo & $\mathrm{Z}$ & Probability \\
\hline & 86.6 & 48.7 & 2.489 & $0.013^{*}$ \\
Entrance x-section $\left(\mathrm{cm}^{2}\right)$ & 6.6 & 11.5 & 1.459 & 0.147 \\
Hole depth $(\mathrm{cm})$ & 222.2 & 263.7 & 0.339 & 0.735
\end{tabular}

* Significant difference

Examination of the size distribution of entrance holes of cuckoo parasitised and unparasitised yellowhead nests (Figure 7.1) shows clearly that cuckoos do not use holes with small entrances even though they are more common than holes with large entrances. 


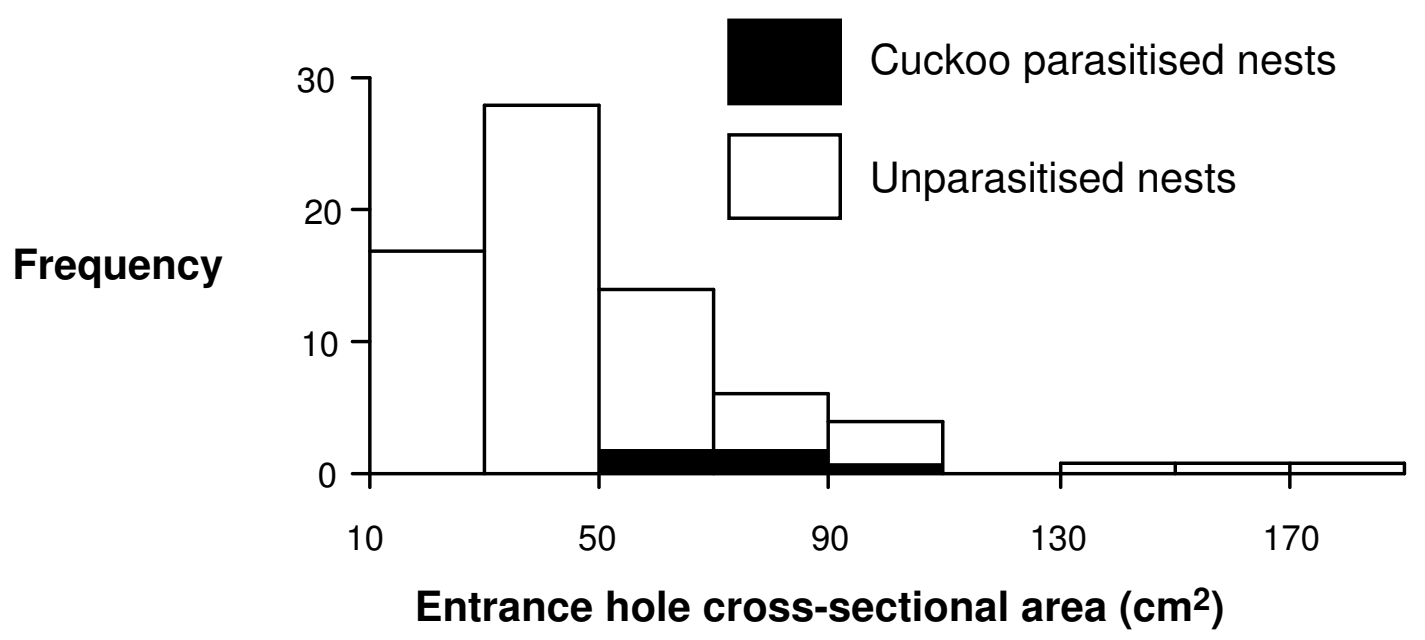

Figure 7.1: A comparison of the entrance hole cross-sectional area of yellowhead nests parasitised by long-tailed cuckoos, with those not parasitised by long-tailed cuckoos.

\subsubsection{Long-tailed cuckoo eggs}

Three of the six nests that were parasitised were found and inspected before any eggs hatched, no long-tailed cuckoo eggs were detected. Oliver's (1955) descriptions of both long-tailed cuckoo and yellowhead eggs indicate that they are almost exactly the same size and only very slightly different in colour. Most cuckoos that parasitise passerines have eggs that mimic those of their hosts (Becking and Snow 1985).

\subsubsection{Laying}

Cuckoos were not seen laying eggs but a few deductions about this event are possible.

The clutch sizes at three cuckoo-parasitised yellowhead nests were 2, 3 and 4 and all hatched only one cuckoo chick. Since clutches of 1 and 2 are very rare amongst normal yellowhead clutches (see Chapter 5), it is likely that cuckoos remove one yellowhead egg when they lay their own. The removal of one host egg and laying of only one cuckoo egg per nest is the norm for cuckoos, though the laying of more than one parasite egg per host nest has been recorded from some cuckoos including the longtailed cuckoos congener the koel (Eudynamis scolopacea) (Becking and Snow 1985).

\subsubsection{Timing of egg laying}

The date at which cuckoo eggs were laid was not known at any of the nests, but the date at which incubation of the nests began is known or was estimated from the hatching dates of yellowhead eggs. Incubation of the earliest and latest clutches that contained 
cuckoo eggs began on about 31 October and 4 January respectively. Figure 7.2 shows the distribution of dates at the beginning of incubation for yellowhead nests with and without cuckoos.

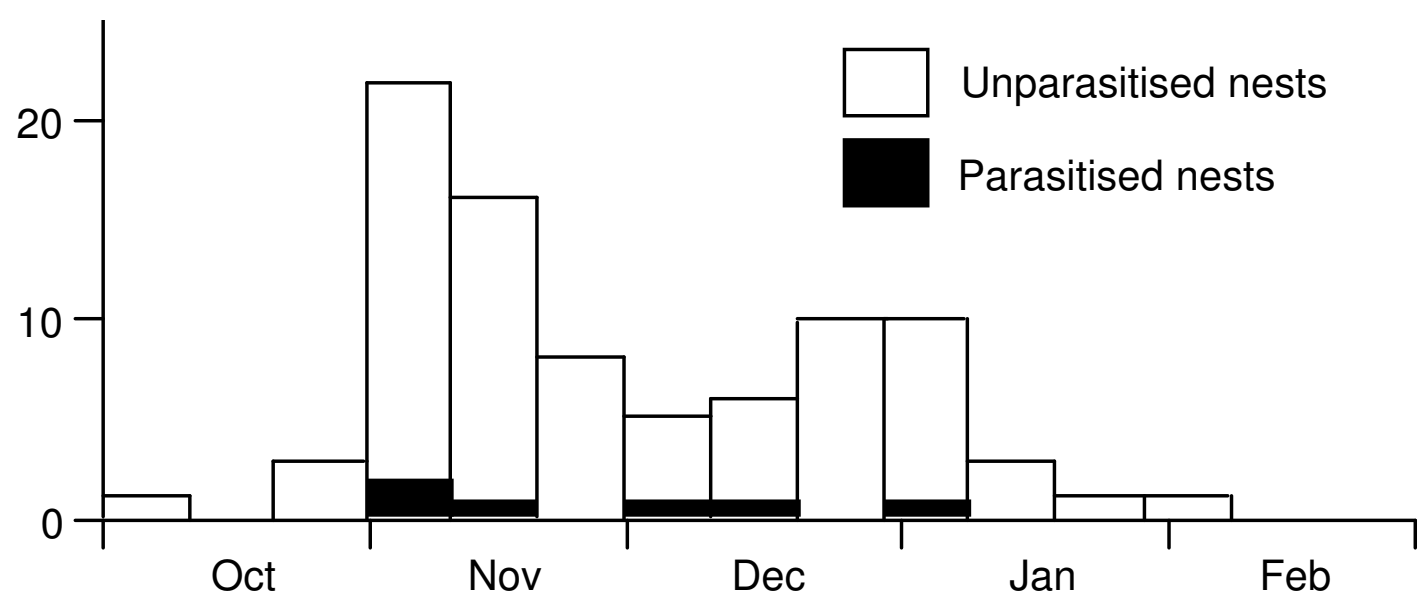

Figure 7.2: $\quad$ The beginning of incubation at cuckoo-parasitised and unparasitised yellowhead nests.

\subsubsection{Hatching and nestling period}

The relative timing of yellowhead and long-tailed cuckoo hatching was known only at one nest where a yellowhead chick hatched about a day before a long-tailed cuckoo. Cuckoos usually hatch before their hosts (Payne 1985).

Recently hatched long-tailed cuckoos are easily distinguished from recently hatched yellowheads. Both hatch naked, but the skin colour of yellowheads is pink, whereas that of cuckoos is dark brown. Cuckoos have much wider mouths which are orange inside. They hatch blind, and beg when disturbed, but when older they hiss, snap and lunge at disturbances. Becking and Snow (1985) suggest that these responses, which are also found in other cuckoos, are adaptations to deter predators.

One cuckoo spent 26 days in the nest before fledging, and appeared to be 2 or 3 days old when I found it. At another nest a long-tailed cuckoo was still in the nest 22 days after hatching. At yet another nest a cuckoo was well covered in feathers when first found, yet spent a further 18 days in the nest before fledging. The nestling period of long-tailed cuckoos is certainly longer than the 21 days which is the maximum length that they have previously been recorded in the nest (Fulton 1904), and is probably about 29 days. Yellowheads fledge in about $22^{1} / 2$ days (see Chapter 5). 
Long-tailed cuckoos are not always able to eject yellowhead chicks from nests. At most nests long-tailed cuckoo chicks pushed the yellowheads up onto the rim of the nest, where they died, but at two nests the shape of the inside of the nesting cavities were such that the yellowhead chicks always fell back in. At both these nests cuckoo chicks were observed trying to eject a yellowhead by wriggling under it and pushing up and backwards, but the yellowhead invariably just toppled off the cuckoo back into the nest. At these two nests both the cuckoo and yellowheads were raised together until the yellowheads fledged. The cuckoos were fed on the nest for at least five days after the yellowhead chick fledged and both cuckoos eventually left the nest but were not seen with their foster parents; they must have died.

For at least the first 11 days of their lives young long-tailed cuckoos were seen attempting to expel yellowhead chicks from their nests. In nests where they were unable to eject the yellowheads, this activity was counter-productive. Not only did it use up energy, but it meant that often when the parent yellowheads arrived at the nest the cuckoo was underneath the yellowhead chick and missed being fed. At the two nests that had both yellowhead and long-tailed cuckoo chicks the cuckoo's development was initially much slower than the yellowheads. By their seventeenth day long-tailed cuckoos chicks had lost the "urge" to eject other nest occupants, they wasted no energy on it and their development appeared to speed.

\subsubsection{Fledging to independence}

Only one cuckoo was seen soon after fledging and this bird was on the ground and unable to fly. It hid between a fern (Polystichum vestitum) and a $\log$ and called quietly to its foster parents. A long-tailed cuckoo chick observe about 22 days after it fledged, perched in one place and waited for its foster parents to come and feed it. I did not observed it feeding itself. This contrasts markedly with yellowhead chicks which immediately after fledging sit and wait to be fed, but as they develop they increasingly follow their parents around. By 22 days after fledging young yellowheads follow their parents all the time. McLean (1982) also observed well developed cuckoos sitting waiting to be fed by whiteheads rather than following them.

Two long-tailed cuckoos were observed being fed 22 and 28 days after they fledged, the latter of which was not being fed at 33 days. Another cuckoo was no longer fed by its foster parents after 32 days. From this limited information it is apparent that the maximum time that cuckoos are fed off the nest is between 28 and 32 days. 


\subsubsection{Nest predation}

I saw long-tailed cuckoos robbing a yellowhead nest only once. A cuckoo was first seen reaching into a nest hole and pulling the feathers off the back of the almost fully fledged nestlings inside. The cuckoo fled when I approached, but 90 minutes later was seen on the ground about $10 \mathrm{~m}$ from the nest thrashing a small bird. When inspected next morning the nest was empty.

Within my study area I also observed long-tailed cuckoos eating a nestling robin and a nestling chaffinch (Fringilla coelebs). Both nestlings were almost of adult size and both were being thrashed on the ground by a cuckoo.

Cuckoos were seen inspecting two further yellowhead nests, though both subsequently fledged young.

\section{DISCUSSION}

\subsubsection{Laying of cuckoo eggs}

Adult long-tailed cuckoos must be able to squeeze into nests they lay in since their fledglings, which are only slightly smaller are able to leave them. However the inside dimensions of most nest cavities are much less than the length of a cuckoo, and it would be difficult for an adult cuckoo to squeeze in, lay, and leave. Some cuckoo species get their eggs into host nests by laying them above a nest and letting them fall into it (Becking and Snow 1985). It is possible that long-tailed cuckoos also do this. Early ornithologists (e.g. Fulton 1904) believed that cuckoos often laid their eggs on the ground and transferred them to their host's nest in their bills. This view has long been discredited (Cunningham 1949).

\subsubsection{The timing of yellowhead and cuckoo laying}

None of the earliest or latest yellowhead nests at Knobs Flat were parasitised (Figure 7.2). However, Lovegrove (1985) and Wilkinson \& Wilkinson (1952) report longtailed cuckoo chicks that must have been laid much later than the last yellowhead eggs at Knobs Flat, and it is likely that the latest nests that I recorded are vulnerable to cuckoos. Nests at which incubation began before November, however, are probably never parasitised since cuckoos are generally supposed not to arrive in New Zealand before October (Cunningham 1985). I have not detected them at Knobs Flat before 18 October, and they may not be able to lay straight away. 
The first clutches of grey warblers completely escape nest parasitism because they are laid before the shining cuckoo (Chrysococcyx lucidus) arrives (Gill 1983). Although a few yellowhead nests are probably too early for long-tailed cuckoos, the bulk of first clutches are not.

\subsubsection{Fledgling cuckoo behaviour}

The fact that fledgling long-tailed cuckoos do not follow their foster parents may explain the apparent deaths of the two cuckoos that were raised with yellowhead chicks. The cuckoos would not have left their nests until 6-7 days after the yellowheads fledged, by which time the yellowheads would have started following their parents around. Thus the cuckoos would be sitting waiting to be fed whereas the young yellowheads would be harassing their parents for food. It is conceivable that the yellowheads continued to feed cuckoos on the nest after yellowheads had fledged because they were attached to the nest site. Once both yellowhead and cuckoo chicks were out of the nest, however, no special attempt would have been made to feed the cuckoo, consequently the yellowhead chicks would have got most of the food.

\subsubsection{The rate of nest parasitism and its effect on yellowhead productivity}

Six of 95 nests that I studied were parasitised; a rate of $6.3 \%$. The effect on yellowhead productivity was less than $6.3 \%$ because one third of the parasitised nests still produced yellowheads. The average number of fledglings produced at nests without cuckoo parasitism was 1.19 per nest. At 95 nests without parasitism one would therefore expect a total of 113.09 chicks, whereas with parasitism one would expect 107.95, a reduction of $4.55 \%$.

The fact that I saw long-tailed cuckoos at three nests yet I spent relatively little time watching nests, suggests that long-tailed cuckoos inspected many more that I did not see. Of the 95 nests I monitored, I had evidence that 9 (9\%) were known of by cuckoos (6 parasitised, 1 robbed and 2 inspected). I believe the cuckoos knew of many more.

Long-tailed cuckoos are known to rob nests of eggs as well as nestlings (Oliver 1955, Soper 1976), and given that long-tailed cuckoos knew the whereabouts of many yellowhead nests it seems likely that they would have robbed any they were not able to parasitise. At the one nest from which I saw yellowhead chicks being removed by a cuckoo there was no sign of disturbance, nor any remains of the chicks. In contrast, the other main predator of yellowhead nests, stoats, often left eggshell fragments or bones 
and feathers in the nest. Eight of the 95 nests that I monitored were abandoned when some or all of the eggs or chicks disappeared without trace, and it is likely that at least some of these were due to long-tailed cuckoo predation.

If cuckoos only preyed upon the one nest that I saw being robbed, then the reduction in yellowhead productivity due to both cuckoo parasitism and predation is $5.62 \%$. If the 8 nests described above were also taken by cuckoos then the reduction in productivity caused by cuckoos is $15.28 \%$.

\subsubsection{Does hole nesting protect yellowheads from long-tailed cuckoos?}

The implication of Figure 7.1 is that the entrance holes of $62 \%$ of yellowhead nests are so small that they prevent cuckoo brood parasitism. Furthermore the shape of the inside of some nest cavities prevents young yellowheads from being ejected. If yellowheads did not nest in holes and if open nests were parasitised at the same rate as the large hole nests, the effect of nest parasitism would be reduction in yellowhead breeding success of $16.8 \%$ instead of $4.55 \%$.

McLean (1988) calculated a brood parasitism rate for long-tailed cuckoos using whiteheads as hosts on Little Barrier Island. His rate was calculated as "... the proportion of successful Whitehead groups (i.e. those with fledglings) which had a cuckoo fledgling" and was based on observations of groups of whiteheads and their fledglings, not on their nests. On this basis 3 of 45 groups of yellowheads were seen with fledgling cuckoos in my study area, giving a rate of $6.67 \%$. McLean found much higher overall rates of brood parasitism amongst whiteheads (16.5\%), though whitehead nests at low altitudes were parasitised at a rate of $5.4 \%$, and those at high altitudes at $35.7 \%$.

Without knowledge of cuckoo density, direct comparisons between rates of cuckoo parasitism of open nesting whiteheads and hole nesting yellowheads are not meaningful. Similarly my conclusion that hole nesting reduces the effect of nest parasitism in yellowheads from 16.8 to $4.55 \%$ assumes that the availability of yellowhead nests limits cuckoo breeding. None-the-less these results strongly imply that hole nesting by yellowheads reduces cuckoo nest parasitism.

If hole nesting reduces nest parasitism by cuckoos then it must also reduce nest predation by them. Since the other native avian predators, falcons (Falco novaeseelandiae), Australasian harriers (Circus approximans), and morepork (Ninox 
novaeseelandiae) are bigger (or in the morepork's case, wider) than long-tailed cuckoos, hole nesting must also be protection against nest predation by these birds.

This study supports Soper's (1976) suggestion that hole nesting is an adaptation to reduce cuckoo parasitism and predation, and it is likely that it reduces rates of predation by all native predators. However, Alerstam and Högstedt (1981) suggested that hole nesting is only advantageous when the main predators find their prey by sight. The arrival of mammalian predators in New Zealand which find their prey by smell, probably means that hole nesting is no longer advantageous.

\section{CONCLUSIONS}

1. Six of 95 yellowhead nests found at Knobs Flat in the Eglinton Valley in Fiordland were parasitised by long-tailed cuckoos.

2. Long-tailed cuckoos have a nestling period of about 29 days; much longer than the yellowheads nestling period of about $22^{1} / 2$ days.

3. Long-tailed cuckoos are fed by their foster yellowhead parents for between 28 and 32 days once they leave the nest.

4. The size of yellowhead nest entrance holes probably prevents many yellowhead nests from being parasitised.

5. Nestling long-tailed cuckoos are sometimes unable to eject yellowhead nestlings and long-tailed cuckoos are sometimes raised alongside yellowheads.

6. Because yellowheads fledge before long-tailed cuckoos, cuckoos do not survive when they are raised alongside yellowheads.

7. Long-tailed cuckoos rob yellowhead nests of nestlings and probably eggs. They also take nestling robins and chaffinches.

8. The net effect of long-tailed cuckoo parasitism and predation on yellowheads is a reduction in productivity of between 5 and $15 \%$. 
9. Hole nesting probably provides significant protection from parasitism by longtailed cuckoos and predation by cuckoos and other avian predators. However it may make them more vulnerable to introduced mammalian predators. 
SECTION 3

\section{HABITAT RELATIONSHIPS}




\section{CHAPTER 8}

\section{HABITAT PREFERENCE}

\section{1}

INTRODUCTION

In the preceding chapters I have demonstrated that introduced predators have probably caused all yellowhead populations to decline, and the decline in low productivity populations has been such that many are extinct or nearly extinct.

The aim of this chapter is to test the hypothesis that the places where yellowheads have remained common have always supported larger, more productive populations than the places where they are rare or from which they have disappeared. Such a study will have considerable consequences for the future management of yellowheads. If yellowheads are simply disappearing from "inferior" habitats, then conservation effort would best be focussed on the remaining good habitats where the birds have better chances of survival. If this is not the case and yellowheads have disappeared from some places for reasons specific to those places, then effort might better be directed at finding out these reasons and acting accordingly.

To test this hypothesis I constructed a "habitat suitability index" which is a statistically constructed scale of habitat quality based on the yellowhead's patterns of habitat preference (Verner et al. 1986). It assumes that yellowheads prefer to live in habitats with the highest carrying capacities (Berry 1986), and habitats in which their productivity is greatest (Brennan et al. 1986). Using this scale I compare habitats in the southern South Island which still have yellowheads with habitats in the northern South Island from which yellowheads have disappeared or become rare.

Read (1988) studied the habitat preferences of yellowheads in Arthur's Pass National Park and observed that yellowheads apparently prefer forests on steeper slopes, and forests with large trees and red beech trees. He hypothesised that large trees contained a greater variety of habitats for invertebrates and thus might support better faunas than did small trees. He also noted that red beeches are known to occupy more fertile sites, and that this might lead to a greater productivity within red beech forest, and consequently a larger invertebrate biomass. Elliott and Ogle (1985), also observed relationships between yellowhead distribution and topography, soil fertility, forest structure, and forest composition.

Following Read (1988) and Elliott \& Ogle's (1985) work I constructed a habitat suitability index based on topography, forest structure, forest composition, and nutrient 
levels. I assumed that prey abundance, nest site abundance and consequently yellowhead abundance and productivity were related to this index.

Another approach to testing this hypothesis would be to compare the density and productivity of yellowheads in places where they remain with places from which they have disappeared or are about to disappear. Productivity and density measures already exist for one population in the Eglinton Valley in Fiordland (Chapter 5), but none exist for populations that have become extinct, nor for populations that seem likely to become extinct in the near future. Furthermore such studies are time consuming, expensive, and of course for extinct populations, impossible.

Another factor that may have an influence on the habitat preferences of yellowheads is the presence of brown creepers. Brown creepers and yellowheads belong to the same family and probably the same genus (Sibley \& Ahlquist 1987), and they appear to forage in a similar manner. The effect each has on the distribution of the other is explored in the construction of a habitat suitability index.

There were two main stages in meeting the main aims of this chapter:

1. Constructing a habitat suitability index.

2. Comparing habitats using the habitat suitability index.

The habitats that were compared were those within the study area where the habitat suitability index was constructed, and a selection of sites in the northern South Island where yellowheads once occurred or still occur.

\section{2}

\section{STUDY AREA}

\subsubsection{Habitat suitability index study area}

The habitat suitability index was constructed from data collected in the Dart, Rees and Routeburn catchments in Mount Aspiring National Park (Figure 8.1). A suitable study area had to contain a variety of habitats some of which contained yellowheads and some of which did not. Furthermore, the places without yellowheads had to lack them because of an unsuitable environment, rather than because they never reached the area, or disappeared through some historical accident. A suitable study area would therefore consist of a large block of continuous forest which contained a wide range of forest types, and in which yellowheads were patchily distributed. The Dart, Rees and Routeburn catchments met these criteria.

The forests and climate of Mount Aspiring National Park have been described in detail by Mark (1977). Rainfall within the study area ranges from about $3000 \mathrm{~mm}$ per annum on the main divide in the west, to about $1000 \mathrm{~mm}$ in the lower reaches of the Rees 
valley in the east. The forests are simple in both structure and diversity. They are dominated by three species of southern beech: silver beech, red beech, and mountain beech (Nothofagus solandri var. cliffortioides). At low altitudes all three species occur but red beech dominates. With increasing altitude the red beech becomes less important, and above $700 \mathrm{~m}$ it is absent. In wetter western areas silver beech forms the dominant subcanopy species under red beech and replaces it at high altitude, whereas in the east this role is taken by mountain beech. The largest trees are red beech, which in the valley floors achieve diameters of $2 \mathrm{~m}$ and heights of $40 \mathrm{~m}$. At the tree line, at between 1050 and $1200 \mathrm{~m}$, trees are quite stunted, reaching at most only $8 \mathrm{~m}$, with diameters of about $1 \mathrm{~m}$.

The only other common trees are Hall's totara (Podocarpus hallii), which is sometimes a canopy tree but mostly occurs only in the understorey; broadleaf, weeping mapou (Myrsine australis) and mountain toatoa, which are understorey plants; and several small-leaved Coprosma species which are rarely more than $2 \mathrm{~m}$ high.

\subsubsection{Sites for comparison}

Figure 8.2 shows the location of sites in the northern South Island which were used for comparison. These sites included one which still had yellowheads, three from which yellowheads had been recorded in the last five years, and 2 with no yellowheads that had similar landforms and forest types to places within Mt Aspiring National Park with high yellowhead densities, and at which yellowheads had previously been recorded. 


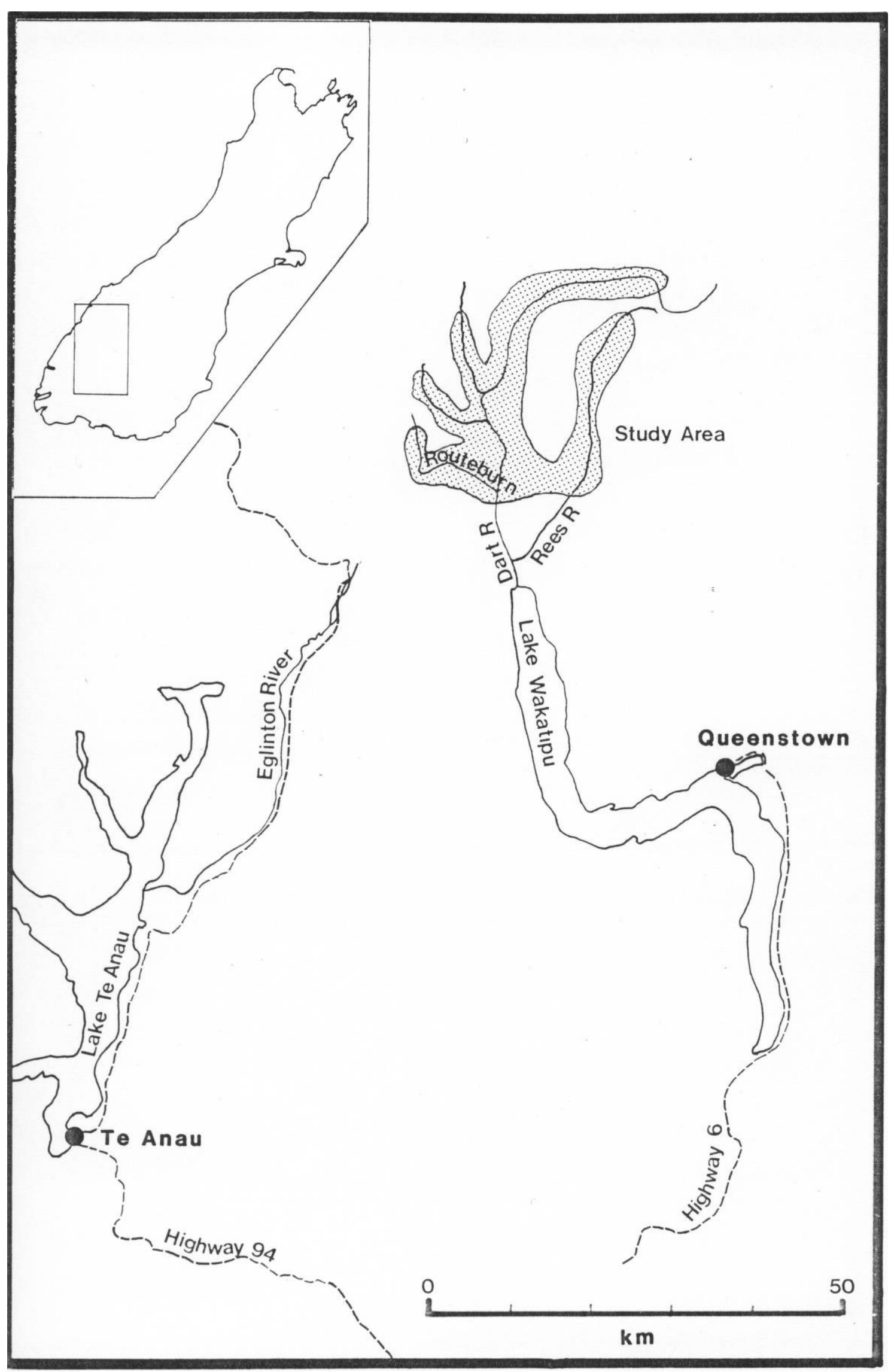

Figure 8.1: $\quad$ Habitat suitability index study area. 


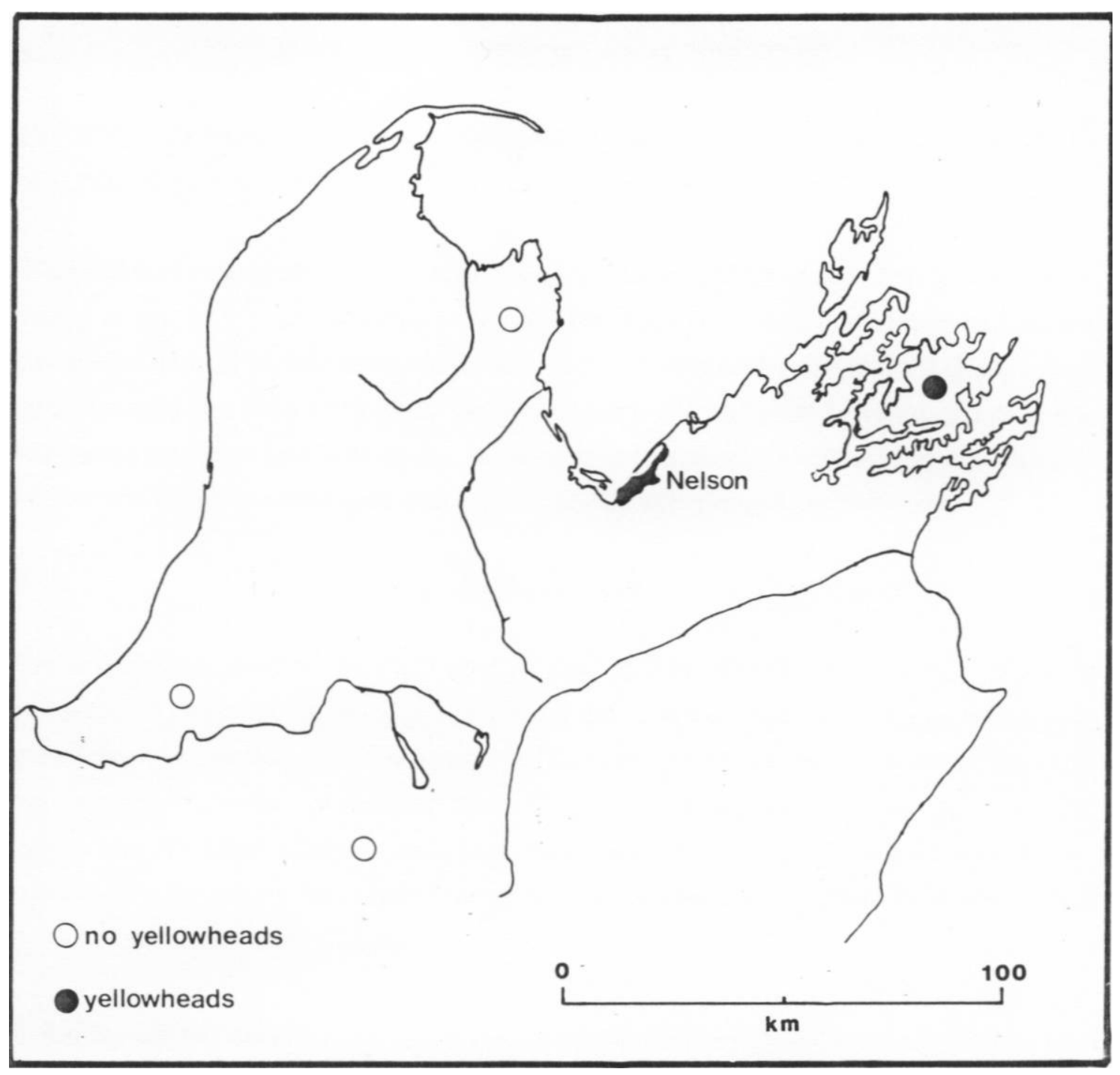

Figure 8.2: $\quad$ Sites in the northern South Island where the habitat suitability of beech forests for yellowheads was assessed. 
The study was undertaken between 15 October and 30 November 1986. At this time of year yellowheads are territorial and vocal and are resident in the areas in which they nest.

The field work was carried out by myself and 80 supervised Operation Raleigh venturers: young adults on a youth adventure course. The field work was undertaken in four ten day spells, with 20 people involved in each spell. During each ten day spell, 3 days were spent training the field workers to identify forest birds and plants, and to carry out the vegetation measurement techniques. In the field the venturers operated in groups of three or four and where possible each group contained an experienced ornithologist.

\section{4}

METHODS

The survey was based on the 1000 yard (914 m) grid on NZMS 1 maps (scale 1:63360), and an attempt was made to collect data from the centre of every grid square which was predominantly forested. Initially I proposed that each square be visited twice but time and manpower constraints prevented this. Centres of grid squares were located only approximately using compass and map, and where the centre of the grid square was inaccessible the survey was carried out as near to the centre as possible. Four sets of data were recorded from each grid square visited.

\subsubsection{Bird data}

A list of birds that were seen or heard within $200 \mathrm{~m}$ of the centre of the square was recorded. An attempt was also made to count the number of groups of yellowheads within $200 \mathrm{~m}$ of the centre of the square, and to count the number of yellowheads within each group.

The time of arrival at the centre of the square, and the time at which yellowheads and brown creepers were first heard or seen was also recorded. This enabled standardisation of the amount of time spent looking for birds: birds first recorded after a standard length time could be ignored in later analysis. At least 45 minutes was spent at the centre of each square.

\subsubsection{Topography}

At the centre of each square the following topographic data were recorded: 
1. Aspect; determined by taking a compass bearing with the compass facing away from the slope of the hill.

2. Slope; measured using a protractor and plumb-bob.

3. Landform; categorised into:

a. valley floor or terrace.

b. slope of hill.

c. ridge crest.

d. gully.

e. top of hill.

4. $\quad$ Altitude

5. $\quad$ Grid reference

\subsubsection{Vegetation}

The vegetation at the centre of each square was quantified using the variable area plot method of Batcheler and Craib (1985). Using this method, stem densities (the number of tree stems per hectare) and basal areas (the cross sectional area of tree stems per hectare) were calculated for every woody plant species recorded within each plot. Basal area and stem densities were calculated separately for plants less than two metres high.

\subsubsection{Nutrient levels}

From the silver beech tree nearest to the centre of the vegetation plot, a cupful of live leaves was collected from about head height, placed in a plastic bag and frozen as soon as possible. The leaves were later dried at $40^{\circ} \mathrm{C}$ for 24 hours, ground to a fine powder and the levels of ammonium-nitrogen, potassium, phosphorous, calcium, sodium, chlorine, sulphur, magnesium, manganese, iron, aluminium, copper and zinc measured. Ammonium nitrogen was measured by digesting $0.2 \mathrm{~g}$ of dried, ground leaf in sulphuric acid in the presence of a catalyst ("Kjeldahl digestion") and measuring the ammonia concentration with a probe connected to a microanalyser. All the remaining nutrients were measured by $\mathrm{x}$-ray fluorescence of $5 \mathrm{~g}$ of dried, ground leaf pressed into a round pellet shape.

\section{5}

\section{RESULTS}

\subsubsection{Coverage}

There were 354 forested 1000 yard grid squares within the study area, of which 259 (73\%) were surveyed once and 154 (44\%) surveyed twice: 413 observations in total. Figure 8.3 shows the coverage. Most of the squares that were not visited had only a small amount of forest within them, or they were on country so steep that access was impossible. Even though some squares were surveyed twice, the imprecision in 
locating the centre of squares was so great that it is unlikely that two samples were ever taken from exactly the same place. For this reason I treat all observations as independent random samples and include all in the analysis.

\subsubsection{Patterns of yellowhead distribution}

Yellowheads were widely distributed in the study area (Figure 8.3). They were most common in the lower reaches of the Dart River and in the Routeburn Valley. They were conspicuously less common in the Rees Valley and in the head-waters of the Dart.

To characterise yellowhead habitat, all of the vegetation and topographic parameters were tested for differences between those sites that had yellowheads and those that did not. For continuous variables the $\mathrm{t}$ test was initially used, but where the data were not normally distributed the Kruskal-Wallis test was used (Table 8.1). Discontinuous variables were tested using contingency table analysis (Table 8.2). Though slope and aspect were recorded in the field as continuous variables, they were grouped and treated as discontinuous variables for analysis. Slope and aspect were each divided into four classes, $<5^{\circ}, 5-15^{\circ}, 15-25^{\circ}$ and $>25^{\circ}$, and north, south, east and west, respectively. Though five landform classes were originally recognised, the frequency at which some landforms were recorded was too small to be used in contingency table analysis; consequently the original five landform classes were re-classified as either valley floor or "other". 


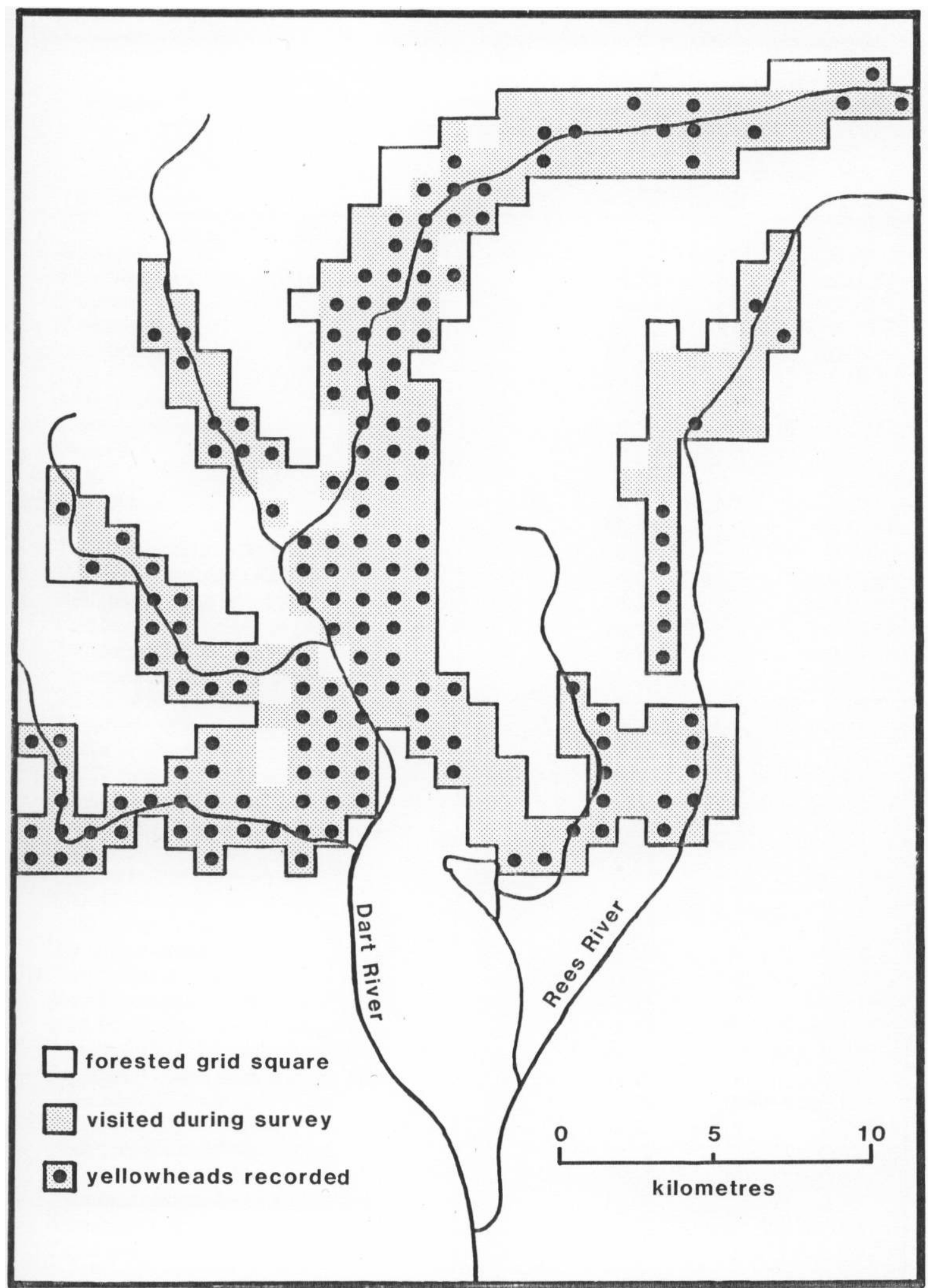

Figure 8.3: Survey coverage and distribution of yellowheads within the study area. 


\section{Table 8.1}

Kruskal-Wallis or t-test test for differences between samples with and without yellowheads. $*$ - significant at the $5 \%$ level, $* *$ at the $1 \%$ level.

\begin{tabular}{|c|c|c|c|c|}
\hline Variable & $\mathrm{t}$ & $\chi^{2}$ & df & Probability \\
\hline Nitrogen & 0.20 & - & 259 & 0.84 \\
\hline Phosphorous & - & 6.83 & 1 & $<0.01 * *$ \\
\hline Sulphur & - & 1.64 & 1 & 0.20 \\
\hline Potassium & - & 1.12 & 1 & 0.29 \\
\hline Aluminium & - & 15.15 & 1 & $<0.01 * *$ \\
\hline Iron & - & 15.91 & 1 & $<0.01 * *$ \\
\hline Manganese & - & 0.60 & 1 & 0.44 \\
\hline Magnesium & - & 4.55 & 1 & $0.03 *$ \\
\hline Copper & - & 2.34 & 1 & 0.13 \\
\hline Zinc & - & 1.19 & 1 & 0.28 \\
\hline Calcium & 0.03 & - & 278 & 0.98 \\
\hline Altitude & - & 36.85 & 1 & $<0.01 * *$ \\
\hline Total basal area $(>2 \mathrm{~m})$ & - & 0.06 & 1 & 0.81 \\
\hline Total stem density $(>2 \mathrm{~m})$ & - & 26.61 & 1 & $<0.01 * *$ \\
\hline Red beech basal area $(>2 \mathrm{~m})$ & - & 34.57 & 1 & $<0.01 * *$ \\
\hline Red beech stem density ( $>2 \mathrm{~m})$ & - & 26.56 & 1 & $<0.01 * *$ \\
\hline Mountain beech basal area $(>2 \mathrm{~m})$ & - & 15.25 & 1 & $<0.01 * *$ \\
\hline Mountain beech stem density ( $>2 \mathrm{~m})$ & - & 21.90 & 1 & $<0.01 * *$ \\
\hline Silver beech basal area $(>2 \mathrm{~m})$ & - & 4.36 & 1 & $0.04 *$ \\
\hline Silver beech stem density (>2m) & - & 2.04 & 1 & 0.15 \\
\hline Hall's totara basal area $(>2 \mathrm{~m})$ & - & 0.14 & 1 & 0.71 \\
\hline Hall's totara stem density (>2m) & - & 0.05 & 1 & 0.82 \\
\hline Mountain toatoa basal area $(>2 \mathrm{~m})$ & - & 1.06 & 1 & 0.30 \\
\hline Mountain toatoa stem density ( $>2 \mathrm{~m})$ & - & 1.03 & 1 & 0.31 \\
\hline Dead tree basal area $(>2 m)$ & - & 1.49 & 1 & 0.22 \\
\hline Dead tree stem density $(>2 m)$ & - & 6.71 & 1 & $<0.01 * *$ \\
\hline Other trees basal area $(>2 m)$ & - & 0.13 & 1 & 0.72 \\
\hline Other trees stem density $(2 \mathrm{~m})$ & - & 0.27 & 1 & 0.60 \\
\hline Total basal area $(<2 \mathrm{~m})$ & - & 0.40 & 1 & 0.53 \\
\hline Total stem density $(<2 \mathrm{~m})$ & - & 0.57 & 1 & 0.45 \\
\hline Red beech basal area $(<2 \mathrm{~m})$ & - & 7.95 & 1 & $<0.01 * *$ \\
\hline Red beech stem density $(<2 \mathrm{~m})$ & - & 7.33 & 1 & $<0.01 * *$ \\
\hline Mountain beech basal area $(<2 \mathrm{~m})$ & - & 0.21 & 1 & 0.64 \\
\hline Mountain beech stem density $(<2 \mathrm{~m})$ & - & 0.16 & 1 & 0.68 \\
\hline Silver beech basal area $(<2 \mathrm{~m})$ & - & 5.59 & 1 & $0.02 *$ \\
\hline Silver beech stem density $(<2 \mathrm{~m})$ & - & 4.51 & 1 & $0.03 *$ \\
\hline Hall's totara basal area $(<2 \mathrm{~m})$ & - & 0.12 & 1 & 0.73 \\
\hline Hall's totara stem density $(<2 \mathrm{~m})$ & - & 0.01 & 1 & 0.91 \\
\hline Mountain toatoa basal area $(<2 \mathrm{~m})$ & - & 0.39 & 1 & 0.53 \\
\hline Mountain toatoa stem density $(<2 m)$ & - & 0.38 & 1 & 0.54 \\
\hline Dead tree basal area $(<2 \mathrm{~m})$ & - & 1.14 & 1 & 0.29 \\
\hline Dead tree stem density $(<2 \mathrm{~m})$ & - & 0.09 & 1 & 0.76 \\
\hline Other trees basal area $(<2 \mathrm{~m})$ & - & 0.05 & 1 & 0.82 \\
\hline Other trees stem density $(<2 \mathrm{~m})$ & - & 0.11 & 1 & 0.74 \\
\hline
\end{tabular}




\section{Table 8.2}

Contingency table analysis testing for association between the presence or absence of yellowheads and some topographical parameters.

\begin{tabular}{l|rcc} 
& $\chi^{2}$ & df & Probability \\
\hline Parameter & 7.62 & 1 & $<0.01^{* *}$ \\
Landform & 7.04 & 3 & 0.07 \\
Aspect & 18.52 & 3 & $<0.01 * *$ \\
Slope & &
\end{tabular}

Sites at which yellowheads were recorded can be characterised as: having high levels of some elements; being at low altitude; having a low total stem density of large trees (>2 $\mathrm{m}$ high); having a high stem density and basal area of red beech; and low stem densities and basal areas of mountain and silver beech. Yellowheads were more frequently found on valley floors than on other landforms, and they were more frequently found on gentle slopes than on steep ones.

To determine whether there were any differences between places with high and low numbers of yellowheads, vegetation and topographic parameters were tested for differences between those sites where more than one group of yellowheads was recorded, and those sites where only one was recorded. Only those parameters that had previously been found to differ between sites with and without yellowheads were tested and none were found to be significantly different (Tables 8.3 and 8.4). For this reason only presence or absence of yellowheads is used in further analysis. 


\section{Table 8.3}

Kruskal-Wallis test for differences between sites where more that one group of yellowheads was recorded and sites where only one was recorded.

\begin{tabular}{l|ccc} 
Variable & $\chi^{2}$ & df & Probability \\
\hline & & & \\
Phosphorous & 0.00 & 1 & 0.95 \\
Aluminium & 0.08 & 1 & 0.78 \\
Magnesium & 0.17 & 1 & 0.68 \\
Iron & 0.19 & 1 & 0.67 \\
Altitude & 3.04 & 1 & 0.08 \\
Total stem density $(>2 \mathrm{~m})$ & 0.02 & 1 & 0.89 \\
Red beech basal area $(>2 \mathrm{~m})$ & 0.33 & 1 & 0.57 \\
Red beech stem density $(>2 \mathrm{~m})$ & 1.42 & 1 & 0.23 \\
Mountain beech basal area $(>2 \mathrm{~m})$ & 2.41 & 1 & 0.12 \\
Mountain beech stem density $(>2 \mathrm{~m})$ & 1.42 & 1 & 0.23 \\
Silver beech basal area $(>2 \mathrm{~m})$ & 0.51 & 1 & 0.47 \\
Dead tree stem density $(>2 \mathrm{~m})$ & 0.00 & 1 & 0.95 \\
Red beech basal area $(<2 \mathrm{~m})$ & 0.00 & 1 & 0.96 \\
Silver beech basal area $(<2 \mathrm{~m})$ & 2.53 & 1 & 0.11 \\
Silver beech stem density $(<2 \mathrm{~m})$ & 2.55 & 1 & 0.11 \\
& & &
\end{tabular}

\section{Table 8.4}

Contingency table analysis testing for association between yellowhead density and some topographical parameters.

\begin{tabular}{l|ccc} 
& $\chi^{2}$ & df & Probability \\
\hline Parameter & 0.56 & 1 & 0.45 \\
Landform & 3.60 & 3 & 0.31 \\
Slope &
\end{tabular}

\subsubsection{Yellowhead and brown creeper distribution}

Contingency table analysis was used to determine whether there was any association between the distributions of yellowheads and brown creepers (Table 8.5). There was none. 


\section{Table 8.5}

Contingency table analysis testing for association between the presence or absence of yellowheads and brown creepers.

\begin{tabular}{|c|c|c|c|}
\hline & & Bro & ers \\
\hline & & Present & Absent \\
\hline & Present & 180 & 143 \\
\hline & Absent & 45 & 45 \\
\hline & & $\begin{array}{l}=0.931 \\
=1 \\
=0.3346\end{array}$ & \\
\hline
\end{tabular}

\subsubsection{Habitat suitability index}

Logistic regression was used to produce a habitat suitability index. Logistic regression is a multivariate statistical technique that fits a sigmoid line to the relationship between a character state dependent variable, such as the presence or absence of yellowheads, and continuous independent variables, such as environmental parameters. Figure 8.4 shows such a relationship for one independent variable.

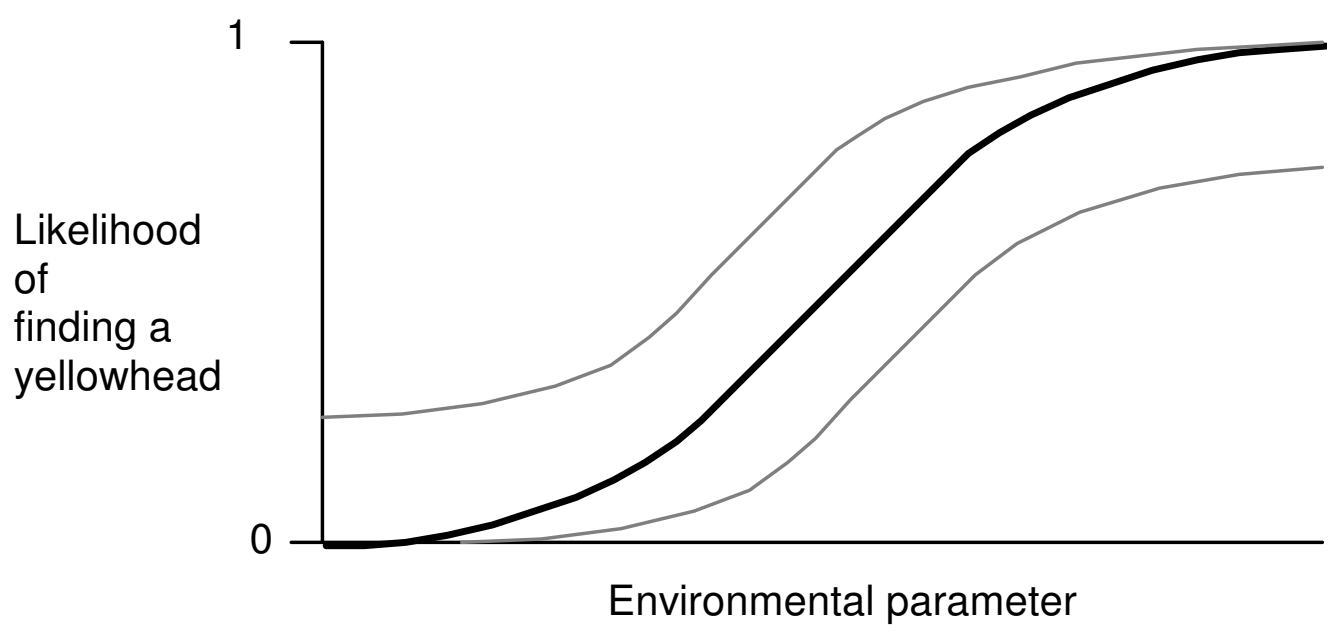

Figure 8.4: $\quad$ Hypothetical logistical relationship between the likelihood of finding yellowheads and some environmental parameter. The broken lines are confidence intervals.

Discriminant function analysis (DFA) can also be used to construct habitat suitability indices from presence/absence data, but this technique was rejected for the following reasons: 
1. DFA assumes that the character states represent multivariate normal distributions. Given that none of the variables that I entered in the model had normal distributions, it is unlikely that my samples came from multivariate normal distributions. Logistic regression is much more robust with respect to departures from normality (Press \& Wilson 1978).

2. DFA constructs linear functions, but given that many ecological phenomena have inherent nonlinear natures, nonlinear statistics such as logistic regression provide more realistic results (Goldstein 1977).

Following Brennan et al. (1986) only those variables that showed significant differences between places with and without yellowheads were used in the model (Tables 8.1 and 8.2). Variables that do not differ significantly between the two classes of sample cannot contribute to the explanatory power of a habitat suitability index.

To further select variables to be added to the model I used the stepwise option of SAS's logistic regression procedure (Harrell 1983). This option successively selects variables that contribute the greatest increase in the explanatory power of the model. Variable selection ceases when there are no more variables that significantly increase explanatory power. Table 8.6 shows the results of the stepwise logistic regression.

The SAS logistic regression procedure is designed for only continuous independent variables. To include the discontinuous variables landform and slope in the analysis, four dummy variables had to be created; one for landform and three for slope. Landform was included with all the other variables in the stepwise logistic regression procedure, but this was not possible with slope because the stepwise procedure is unable to select variables in a group; it selects them separately. To determine whether or not slope should have occurred in the model produced by the stepwise procedure, the logistic regression program was run several times without the stepwise option. Variables were added to the model in the same order as in the stepwise procedure and between successive runs the three slope dummy variables were added to the model and the change in $-2 \log$ likelihood recorded. At no stage did the inclusion of the slope dummy variables cause a greater change in $-2 \log$ likelihood than the addition of the variable picked by the stepwise procedure. I concluded that slope should not be added to the model. 


\section{Table 8.6}

Results of stepwise logistic regression. Variables are listed in the order in which they were added to the model. $* *$ - significant at the $1 \%$ level, $*_{-}$at the $5 \%$ level.

\begin{tabular}{l|rrc} 
& \multicolumn{3}{c}{} \\
Variable & $\beta$ & $\chi^{2}$ & Probability \\
\hline & & & \\
Intercept & 1.9684 & 19.24 & $<0.01^{* *}$ \\
Altitude & -0.0007 & 16.58 & $<0.01^{* *}$ \\
Total stem density $(>2 \mathrm{~m})$ & -2.7362 & 12.39 & $<0.01^{* *}$ \\
Red beech stem density $(>2 \mathrm{~m})$ & 7.5898 & 5.65 & $0.02^{*}$
\end{tabular}

The SAS logistic regression procedure provides a statistic to test how well models fit their data. This statistic indicated that this model did fit the data $\left(\chi^{2}=8.02, \mathrm{df}=9\right.$, $\mathrm{p}=0.5317)$.

These results show that though the distribution of yellowheads is associated with many fertility, forest structure, forest composition and topographic parameters, it is most closely associated with altitude, total stem density of trees and stem density of red beech trees.

\subsubsection{Suitability of northern habitats}

The habitat suitabilities of sites within the study area and in the northern South Island can be calculated by inserting values of altitude, stem density and red beech stem density into the logistic equation. However the effect of altitude on yellowheads is likely to be related to latitude, for example, Wardle (1984) suggested that one degree of latitude shifts the altitudinal distribution of beech forest plants by about $75 \mathrm{~m}$. To correct for this effect I examined topographical maps of Mt Aspiring National Park and the sites of my northern South Island samples and made corrections to the altitudes of the northern South Island samples according to the difference between the tree lines on mountains adjacent the sample sites and the tree line in Mt Aspiring National Park. Figure 8.5 compares the habitat suitabilities in Mt Aspiring National Park with those in the northern South Island. 


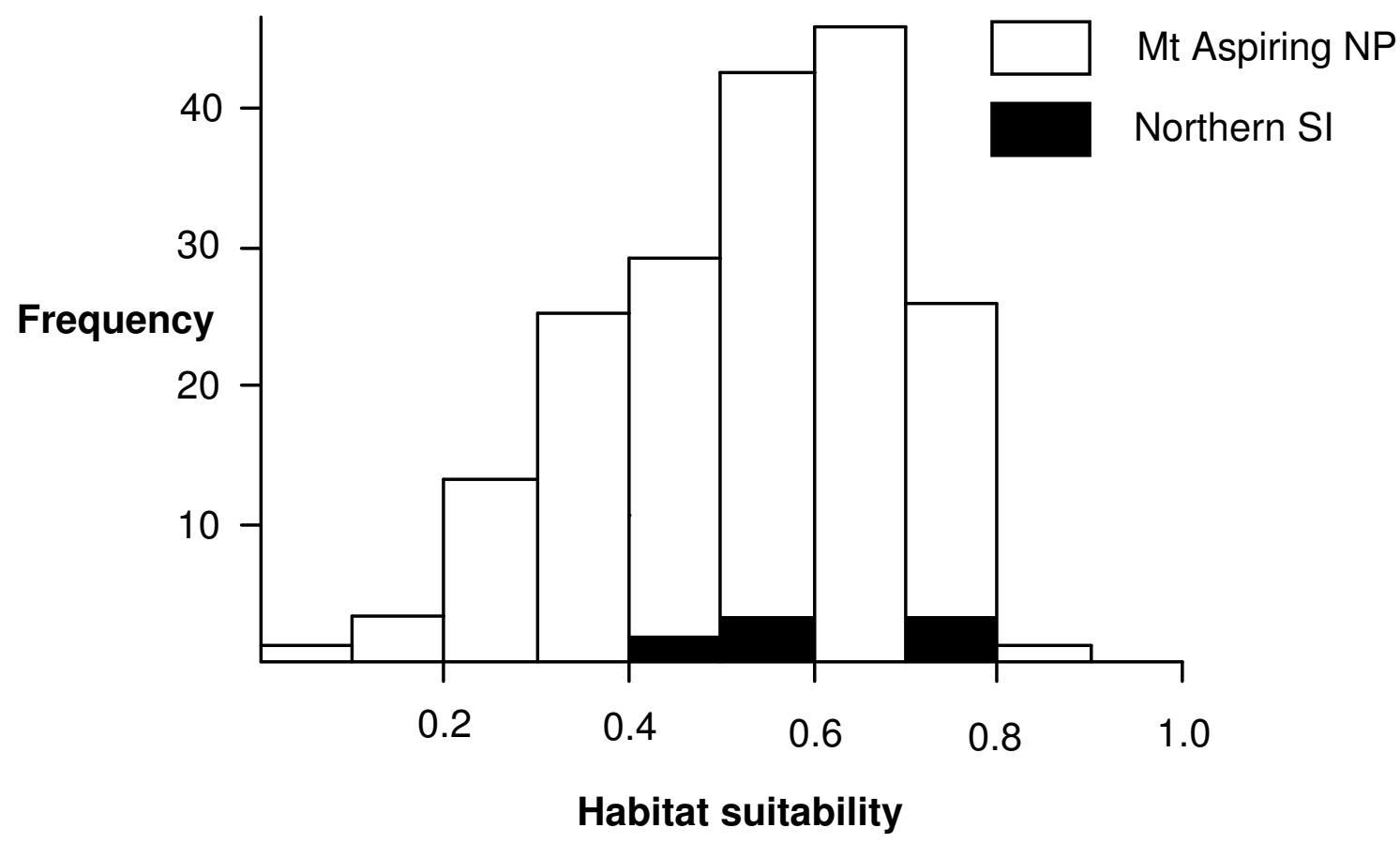

Figure 8.5: Habitat suitability for sites with yellowheads in Mt Aspiring National Park, and sites in the northern South Island.

\section{6}

DISCUSSION

Yellowheads are more likely to be found at sites:

1. at low altitude.

2. with high fertility.

3. on valley floors.

4. with low total stem densities of large trees ( $>2 \mathrm{~m}$ high).

5. with high stem densities and basal areas of red beech.

6. with low stem densities and basal areas of mountain and silver beech.

This study found no evidence of competitive exclusion of brown creepers by yellowheads.

Logistic regression suggests that altitude, stem density, and red beech stem density, in that order, are more closely related to yellowhead distribution than any of the other parameters. The implication of the logistic regression is that yellowhead distribution is determined firstly by factors associated with altitude, secondly by factors associated with forest structure and lastly by factors associated with forest composition. Factors associated with site fertility and landform probably have no direct effect on yellowhead distribution, but are correlated with factors that do. 
The yellowheads apparent preference for forests with low stem densities requires some explanation. As beech forests age their stem density decreases. Young forests are made up of many small trees whereas older forests comprise fewer large trees (Wardle 1984). Stem densities also vary with altitude and forest composition. At high altitudes there tends to be a high density of small trees giving a high stem density, whereas at low altitudes there are few large trees with a low stem density (Wardle 1984). Forests dominated by mountain beech have higher stem densities that those dominated by silver beech, which in turn have higher stem densities than those dominated by red beech (Wardle 1984). The yellowheads apparent preference for low stem densities is consistent with its preference for red beech and low altitudes, but it also suggests that yellowheads prefer mature forests with a few large trees, rather than young forests with many small trees.

The northern South Island sites that were measured were selected because they looked like good yellowhead habitat, i.e., they were forests of large trees of which most were red beech, or because they still had yellowheads or had recently had them. They should have had high habitat suitabilities, and most did (see Figure 8.5). Of particular note is that the only sample from the northern South Island that still has yellowheads (Mt Stokes in the Marlborough Sounds) had one of the lowest habitat suitabilities (0.46).

It is clear that in the northern South Island yellowheads have disappeared from sites that would support good yellowhead populations if they occurred further south. Furthermore, yellowheads seem to have disappeared from some of the best sites, yet survive in some "average" ones. Habitat quality alone cannot explain the disappearance of yellowheads from the northern South Island. 


\section{CHAPTER 9}

\section{HABITAT USE BY YELLOWHEADS}

\section{1}

INTRODUCTION

In Chapter 8 I showed that yellowheads prefer forests with low stem densities (i.e. a few large trees) and dominated by red beech, and these results agree with those of Read (1988a) who worked on yellowheads near Arthur's Pass. The question arises "do these habitat preferences result from yellowheads preferentially using large trees and red beech trees?"

Read (1988a) has already attempted to answer these questions by studying the patterns of habitat use in the forest in which he detected the patterns of habitat preference. Why do it all again?

Read's study area contained almost no silver beech, but silver beech is an important component in forests over most of the range of yellowheads. Its effect on habitat use requires analysis. Furthermore Read's analysis was limited. To detect selective use of forest components one compares their use with their availability. Read (1988a) compared the proportion of time yellowheads spent on different types of tree with the number of trees available measured in stems/ha; he took no account of tree size. Read acknowledged this shortcoming and suggested that a better measure of tree availability would be above ground surface area but noted that there was no practical way of measuring this.

The limitation of Read's study is a common one in studies of avian habitat use (e.g., Noske 1985, Recher et al. 1985) but there are studies that take account of tree size. Holmes and Robinson (1981) used known relationships between tree diameter and leaf area to approximate surface area, and Airola and Barret (1985) estimated cover.

In this study I used three estimates of tree size derived from variable area plots (Batcheler and Craib 1985), and an estimate of above ground surface area derived from a new method I have developed called "point height intercept analysis at an angle" (here-in-after "phiangle"). The novelty of this technique requires that its background and development be described in more detail than is appropriate within this chapter (see Appendix C).

As part of this study I have collected data on yellowhead foraging behaviour and time budget. 


\subsubsection{Sampling yellowhead habitat use}

Yellowhead habitat use was sampled at Knobs Flat using the method adopted by Read (1988a) for study of yellowheads near Arthur's Pass. This method is a modified version of one developed by O'Donnell and Dilks (1988) to measure habitat use by New Zealand forest birds. Groups of yellowhead were followed for periods of up to one hour between 0900 and 1800 hours on fine days and the activity, and position of a bird in the group was recorded every minute on the minute. Different individuals from within the same group were often recorded in successive samples because although it was easy to follow a group, it proved impossible to follow an individual. When the observed bird had been banded and the bands could be read, its identity was recorded.

Yellowhead activities were classed as one of the following:
1. gleaning
2. scanning
3. flying
4. preening
5. manipulating
6. bathing
7. feeding mate or chicks
8. $\quad$ ripping
9. calling

The following details of their position were recorded:

1. tree species

2. diameter at breast height $(\mathrm{DBH})$ of tree

3. height of tree (in $5 \mathrm{~m}$ intervals)

4. height of bird (in $5 \mathrm{~m}$ intervals)

5. perch site

(i) trunk

(ii) large branch $(>30 \mathrm{~cm}$ diameter)

(iii) small branch $(<30 \mathrm{~cm}$ diameter $)$

(iv) twig

(v) foliage

6. location on the $50 \mathrm{~m}$ grid

Most samples were collected during the summer months (November to March), but for comparison a small number were collected during July. 


\subsubsection{Vegetation sampling}

\subsubsection{Variable area plots}

Vegetation within the study area was measured using the variable area plot method of Batcheler and Craib (1985), except that the height of each tree as well as its diameter at breast height $(\mathrm{DBH})$ was measured. Tree height was measured to the nearest $5 \mathrm{~m}$ using a camera with a telephoto lens with a calibrated focusing scale. Batcheler and Craib's method produces measures of stem density and basal area for each plot, and these can be apportioned amongst size classes and species. Trees were divided into 8 size classes: less than $10 \mathrm{~cm}$ DBH, 10-30 cm DBH, 30-50 cm DBH, 50-70 cm DBH, 70-90 cm DBH, 90-110 cm DBH, 110-130 cm DBH, 130-150 cm DBH. In addition estimates of the surface area and volume of trunks in each plot were calculated using the following formulae:

$$
\begin{gathered}
\text { Trunk surface area }=\frac{\pi \infty \mathrm{DBH} \infty \text { height }}{2} \\
\text { Trunk volume }=\frac{\pi \infty \mathrm{DBH}^{2} \infty \text { height }}{8}
\end{gathered}
$$

Seventy four vegetation plots were measured on a $71 \mathrm{~m}$ grid. A $71 \mathrm{~m}$ grid pattern that covered the whole study area was created by measuring vegetation at every second grid point on the $50 \mathrm{~m}$ grid (see Figure 2.5). To provide more accurate quantification of the vegetation in areas where most of the habitat use observations were made, a further 28 vegetation plots were measured halfway between some of those on the $71 \mathrm{~m}$ grid (Figure 9.1).

\subsubsection{Phiangle}

The surface area of vegetation around 18 grid points at Knobs Flat (Figure 9.2) was estimated using phiangle (see Appendix C). This new method of forest mensuration was developed during this study and was not perfected until I had nearly finished. Furthermore, it is very time consuming and I was only able to measure vegetation at a few sites. Only grid points at which many yellowhead habitat use observations had been recorded were chosen for a trial of this technique. 


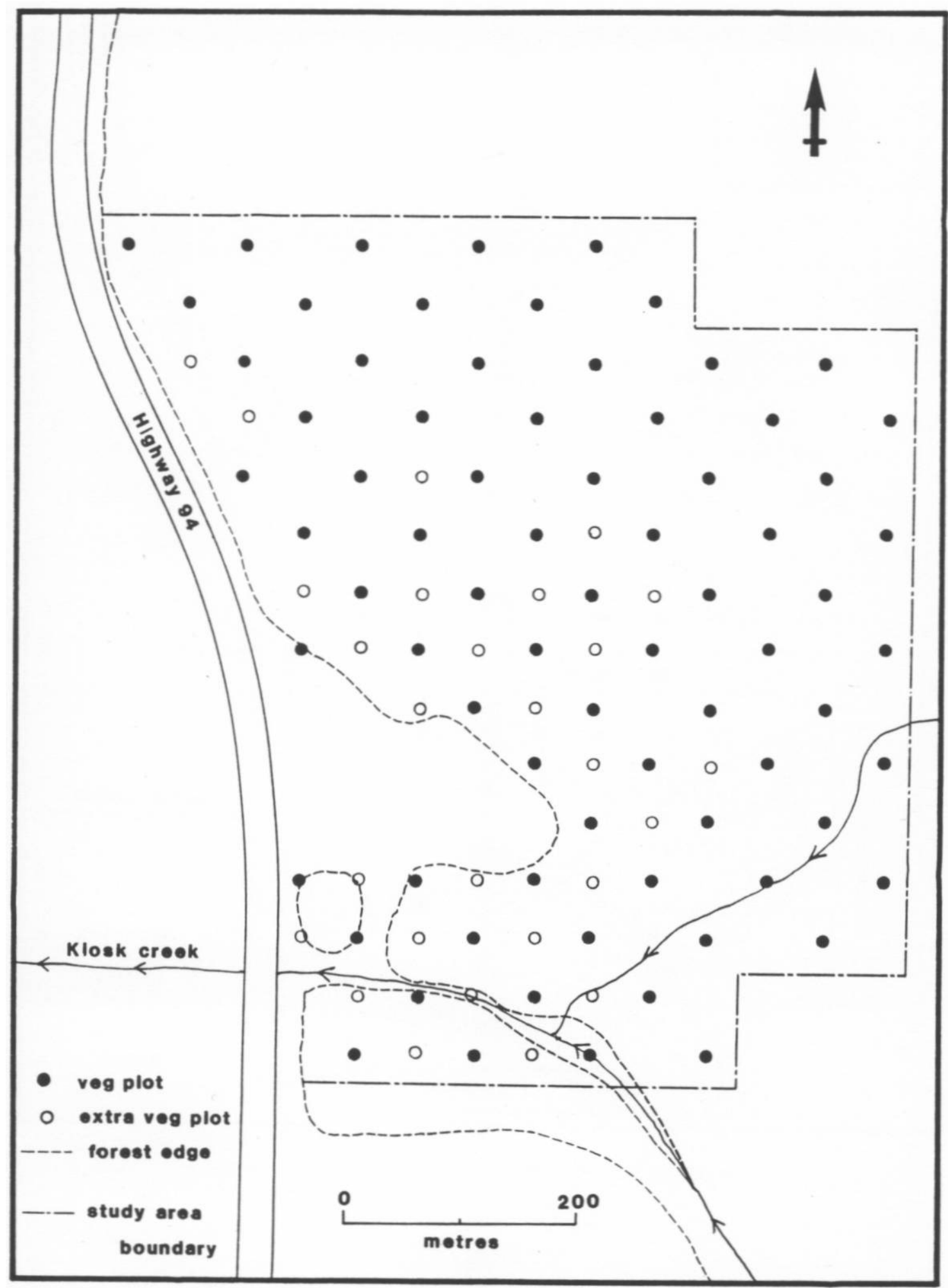

Figure 9.1: $\quad$ Variable area vegetation plots in the Knobs Flat study area. 


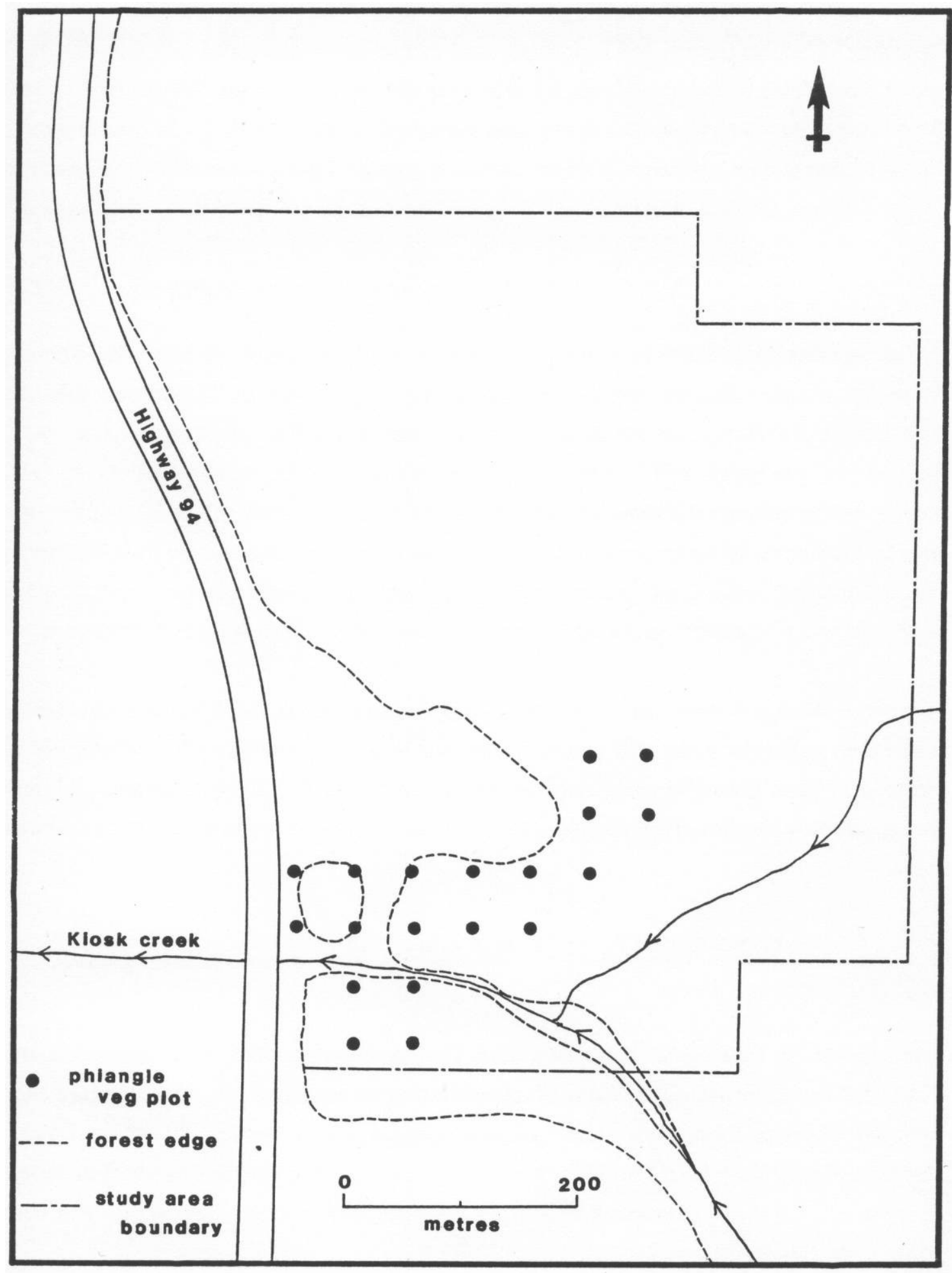

Figure 9.2: $\quad$ Phiangle vegetation plots in the Knobs Flat study area. 
Phiangle provides estimates of the surface area density $\left(\mathrm{m}^{2}\right.$ of surface per $\mathrm{m}^{3}$ of space) of forest. The surface area of a forest can be divided according to any classification using this technique, and I chose to partition surface area into the same categories that were used in measuring habitat use. Surface area was thus divided according to tree species, size classes (DBH), height off the ground, and whether it was branch, trunk or leaves.

\subsubsection{Assessing selective use of trees}

Though yellowheads might be observed more frequently in some sizes and species of tree, this may not reflect selectivity on the part of yellowheads but rather the abundance of these trees. Tests of the null hypothesis that yellowheads are not selective in their use of trees were undertaken using "goodness of fit" tests. The expected number of observations in each species or size class of tree was calculated by assuming that it was proportional to the abundance of trees in that class. Four measures of abundance of tree derived from variable area plots were used; stem density, basal area, estimated trunk volume, estimated trunk surface area, as well as the surface area estimated using phiangle.

Weighted averages of all these measures were used. Averages were weighted according to the number of yellowhead habitat use observations that were recorded near each sample. For example, if 10 yellowhead observations were recorded near vegetation sample site A and 20 near vegetation sample site $\mathrm{B}$, the average basal area was calculated as:

$$
\text { Average basal area }=\frac{(20 \infty \text { basal area of } A)+(10 \infty \text { basal area of } B)}{20+10}
$$

Since variable area plots were measured throughout the study area all habitat use observations could be included in goodness of fit tests involving variable area plot measurements. However, since phiangle measurements were made at relatively few places, only those habitat use observations recorded within $25 \mathrm{~m}$ of the phiangle samples were included in analyses involving phiangle vegetation measures.

\section{RESULTS}

\subsubsection{Yellowhead activities}

1758 samples of yellowhead behaviour in which the activity was determined were recorded.

The proportion of time spent on each activity differed between summer (October to March) and winter (June) $\left(\chi^{2}=183.729, \mathrm{df}=5, \mathrm{p}=0.0001\right)$. In the winter yellowheads 
spent less time gleaning, and on non-feeding activities but more time ripping and probing (Figure 9.3).
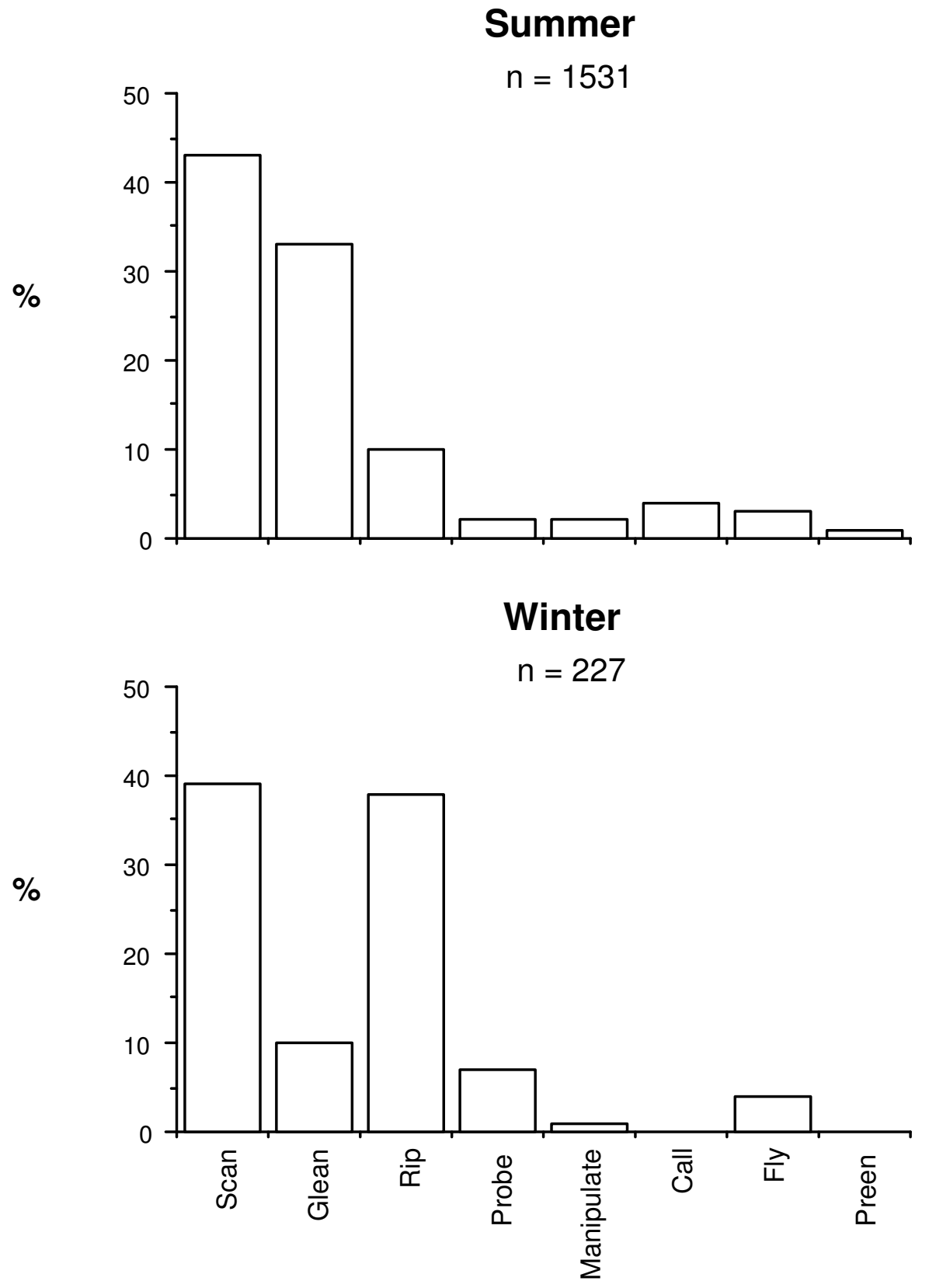

Activity

Figure 9.3: $\quad$ Activity patterns in summer and winter.

Initial inspection of the distribution of observations amongst activities and tree species (Table 9.1) suggests that yellowheads spend a higher proportion of their time gleaning when in mountain and silver beech than they do when in red beech. It is likely that this apparently low incidence of gleaning in red beech results from the fact that gleaning is often carried out in the twigs and foliage in the tops of trees, and as red beech is taller than the other two species, many observations in which the bird was gleaning in red 
beech will have been hidden from view and recorded as unknown. Red beech has a higher proportion of "unknown" activity than the other species.

\section{Table 9.1}

The distribution of observations amongst activity classes and tree species

\begin{tabular}{|c|c|c|c|c|c|c|c|c|c|c|}
\hline \multirow[b]{2}{*}{ Activity } & \multicolumn{2}{|c|}{$\begin{array}{l}\text { Silver } \\
\text { beech }\end{array}$} & \multicolumn{2}{|c|}{$\begin{array}{c}\text { Red } \\
\text { beech }\end{array}$} & \multicolumn{2}{|c|}{$\begin{array}{c}\text { Mountain } \\
\text { beech }\end{array}$} & \multicolumn{2}{|c|}{$\begin{array}{l}\text { Dead } \\
\text { trees }\end{array}$} & \multicolumn{2}{|c|}{$\begin{array}{l}\text { Other } \\
\text { trees }\end{array}$} \\
\hline & $n$ & $\%$ & $n$ & $\%$ & $n$ & $\%$ & $n$ & $\%$ & $n$ & $\%$ \\
\hline Scan & 538 & 29.4 & 139 & 21.3 & 18 & 35.3 & 5 & 27.8 & 39 & 50.6 \\
\hline Glean & 450 & 24.6 & 57 & 8.7 & 12 & 23.5 & 2 & 11.1 & 9 & 11.7 \\
\hline Rip & 175 & 9.6 & 38 & 5.8 & 3 & 5.9 & 7 & 38.9 & 8 & 10.4 \\
\hline Call & 23 & 1.3 & 44 & 6.7 & 0 & 0.0 & 0 & 0.0 & 7 & 9.1 \\
\hline Probe & 34 & 1.9 & 7 & 1.1 & 3 & 5.9 & 2 & 11.1 & 1 & 1.3 \\
\hline Preen & 11 & 0.6 & 8 & 1.2 & 2 & 3.9 & 0 & 0.0 & 2 & 2.6 \\
\hline Roost & 3 & 0.2 & 1 & 0.2 & 0 & 0.0 & 0 & 0.0 & 0 & 0.0 \\
\hline Manipulate & 23 & 1.3 & 4 & 0.6 & 1 & 2.0 & 0 & 0.0 & 3 & 3.9 \\
\hline Feed & & & & & & & & & & \\
\hline (chick or mate) & 6 & 0.3 & 0 & 0.0 & 0 & 0.0 & 0 & 0.0 & 0 & 0.0 \\
\hline Unknown & 568 & 31.0 & 354 & 54.3 & 12 & 23.5 & 2 & 11.1 & 8 & 10.4 \\
\hline$\overline{\text { Total }}$ & 1831 & & 652 & & 51 & & 18 & & 77 & \\
\hline
\end{tabular}

\subsubsection{Yellowhead use of perch sites}

1804 samples of yellowhead behaviour in which the perch site was observed were recorded. There was a strong seasonal difference in the use of perch sites (Figure 9.4) $\left(\chi^{2}=67.4\right.$, df $\left.=3, \mathrm{p}=0.0001\right)$. During the summer most $(56 \%)$ observations were on twigs and foliage whereas in the winter only $28 \%$ were. The use of trunks and branches, particularly small branches, rose in the winter. 


\section{Summer}

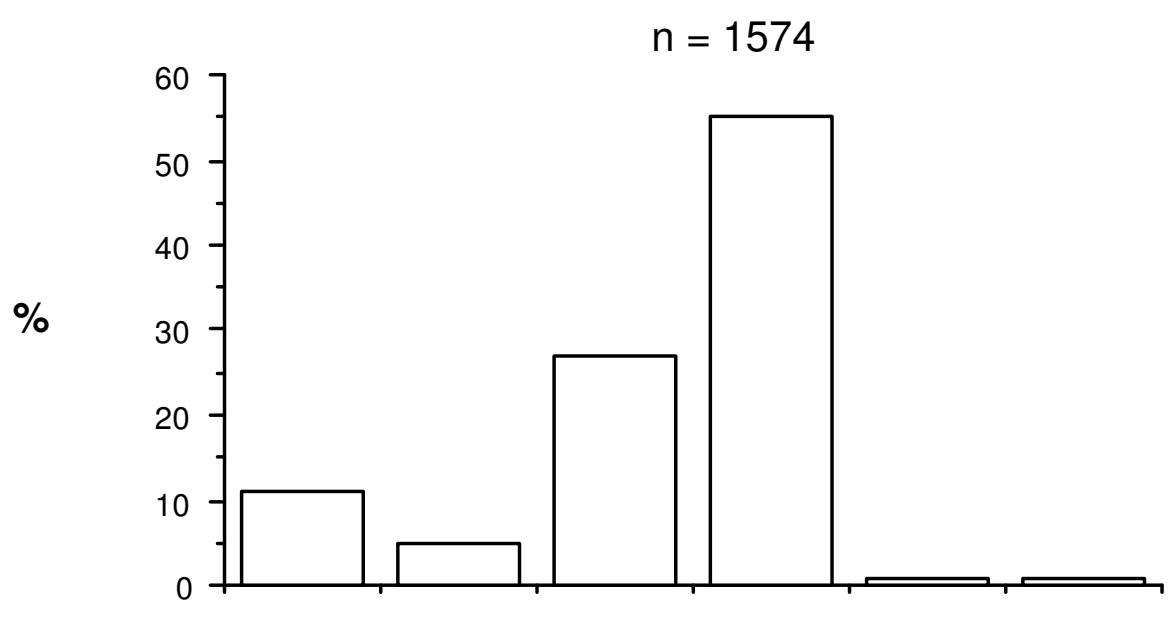

\section{Winter}

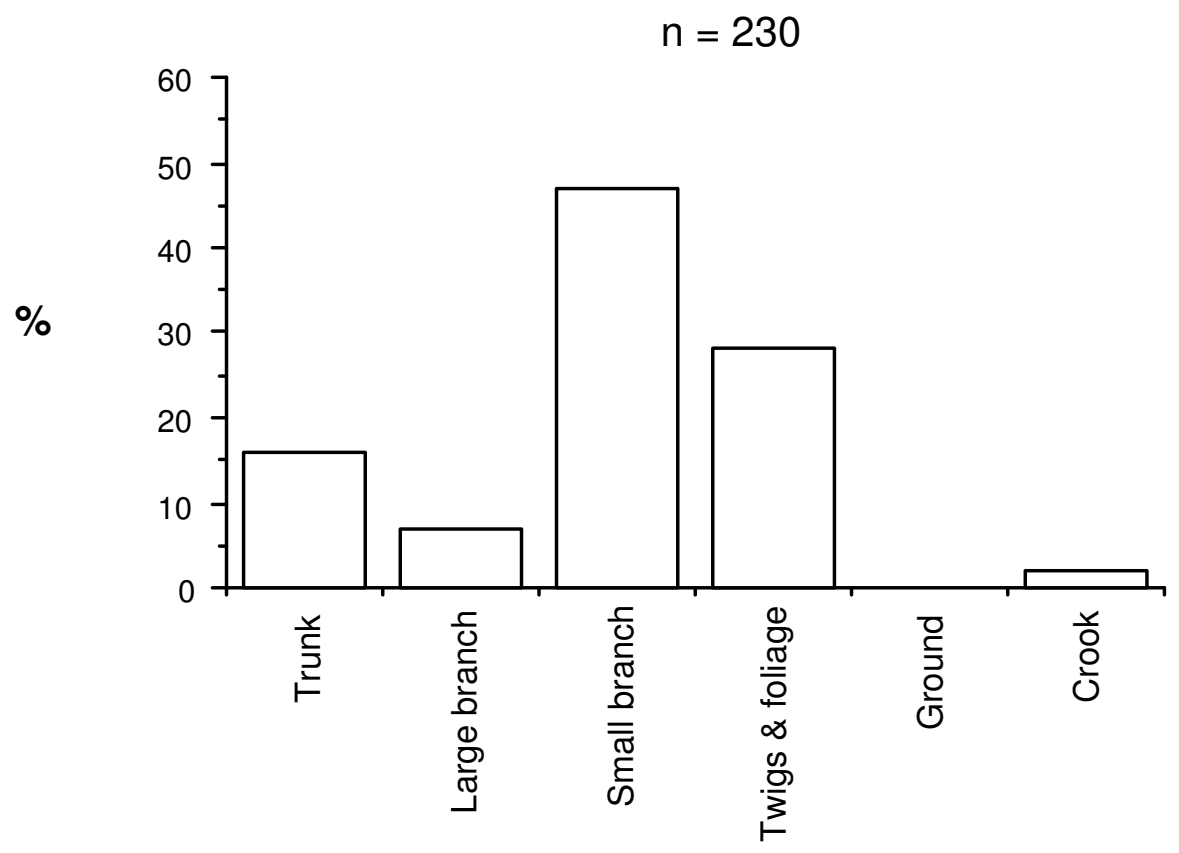

Perch site

Figure 9.4: $\quad$ Use of perch sites in summer and winter.

Like activities, perch sites initially seemed to be differentially distributed amongst tree species (Table 9.2). Once again however, the presence of a large number of observations in which the perch site was not determined, and the bias against recording perch sites in the tops of tall trees, precludes useful analysis. The apparent association between tree species and perch site is probably an artifact of this bias. 
Table 9.2

The distribution of observations amongst perch sites and tree species

\begin{tabular}{|c|c|c|c|c|c|c|c|c|c|c|}
\hline \multirow[b]{2}{*}{ Site } & \multicolumn{2}{|c|}{$\begin{array}{l}\text { Silver } \\
\text { beech }\end{array}$} & \multicolumn{2}{|c|}{$\begin{array}{c}\text { Red } \\
\text { beech }\end{array}$} & \multicolumn{2}{|c|}{$\begin{array}{c}\text { Mountain } \\
\text { beech }\end{array}$} & \multicolumn{2}{|c|}{$\begin{array}{c}\text { Dead } \\
\text { trees }\end{array}$} & \multicolumn{2}{|c|}{$\begin{array}{l}\text { Other } \\
\text { trees }\end{array}$} \\
\hline & $n$ & $\%$ & $n$ & $\%$ & $n$ & $\%$ & $n$ & $\%$ & $n$ & $\%$ \\
\hline Trunk & 160 & 8.8 & 28 & 4.3 & 4 & 7.8 & 8 & 44.4 & 11 & 0.0 \\
\hline Large branch & 68 & 3.7 & 11 & 1.7 & 1 & 2.0 & 2 & 11.1 & 0 & 0.0 \\
\hline Small branch & 351 & 19.2 & 138 & 21.3 & 12 & 23.5 & 6 & 33.3 & 24 & 34.3 \\
\hline Twigs \& foliage & 790 & 43.2 & 117 & 18.1 & 24 & 47.1 & 1 & 5.6 & 19 & 27.1 \\
\hline Unknown & 459 & 25.1 & 353 & 54.6 & 10 & 19.6 & 1 & 5.6 & 16 & 22.9 \\
\hline$\overline{\text { Total }}$ & 1828 & & 647 & & 51 & & 18 & & 70 & \\
\hline
\end{tabular}

\subsubsection{Yellowheads and tree species}

Figure 9.5 shows the distribution of yellowhead observations amongst tree species. Most activity was recorded in either silver or red beech, with nearly three quarters of observations being in silver beech. There was a significant difference in the use of trees in the summer and winter $\left(\chi^{2}=21.705, \mathrm{df}=4, \mathrm{p}=0.0002\right)$ with yellowheads making less use of red beech in the winter. 


\section{Summer}

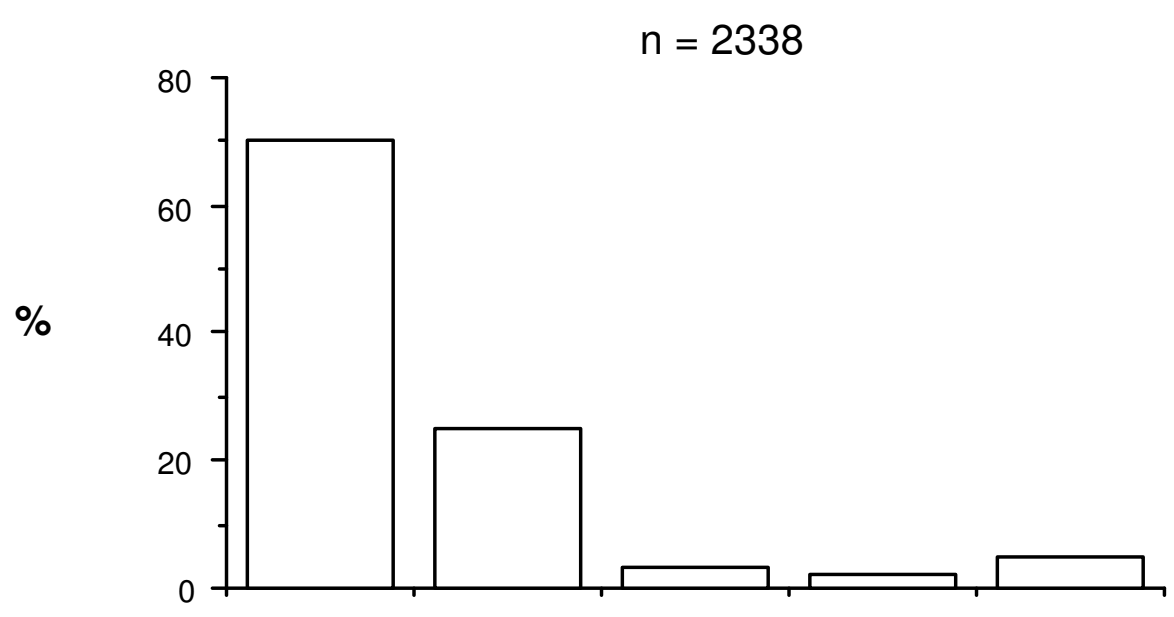

\section{Winter}

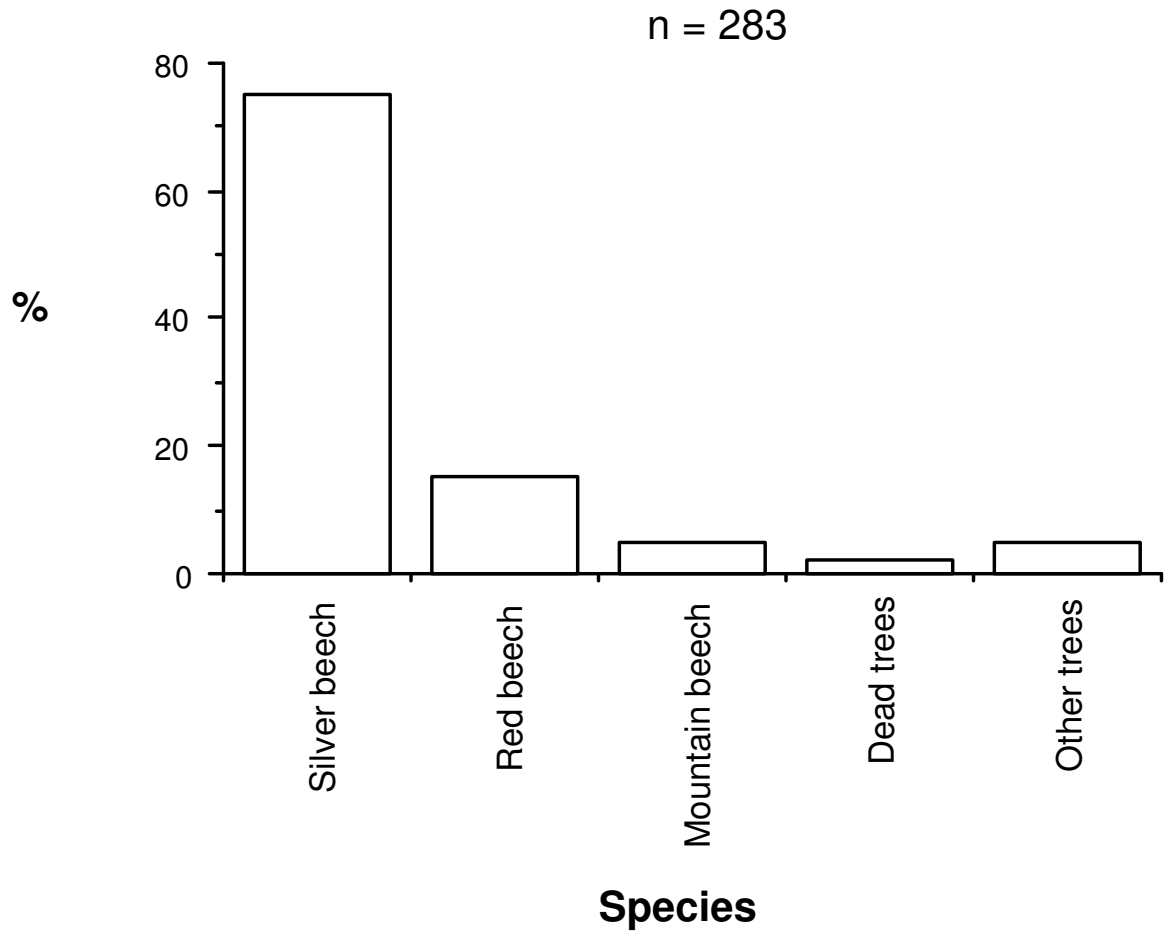

Figure 9.5: $\quad$ Use of different tree species in summer and winter.

\subsubsection{Yellowheads and tree size}

Figure 9.6 shows the distribution of yellowhead habitat use amongst trees of different sizes. Most yellowhead activity was recorded in trees greater than $30 \mathrm{~cm} \mathrm{DBH}$, and use of trees less than $50 \mathrm{~cm}$ DBH was less in the winter than in the summer $\left(\chi^{2}=19.733\right.$, $\mathrm{df}=6, \mathrm{p}=0.0031)$. 


\section{Summer}

$\mathrm{n}=2338$

Percent of observations

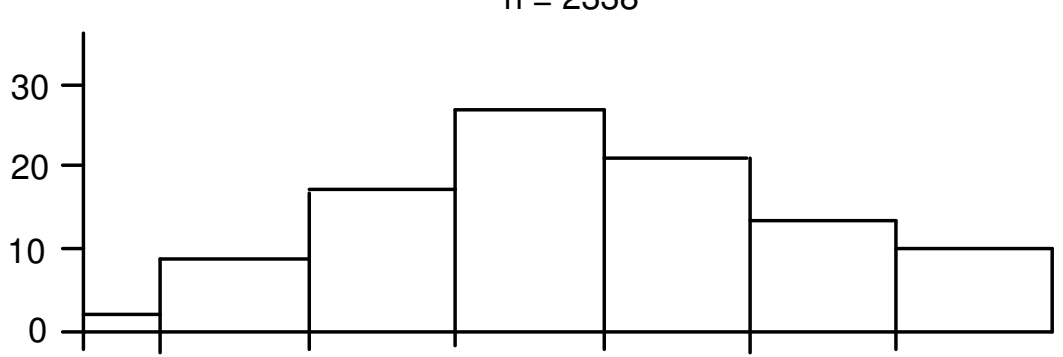

Winter

$\mathrm{n}=283$

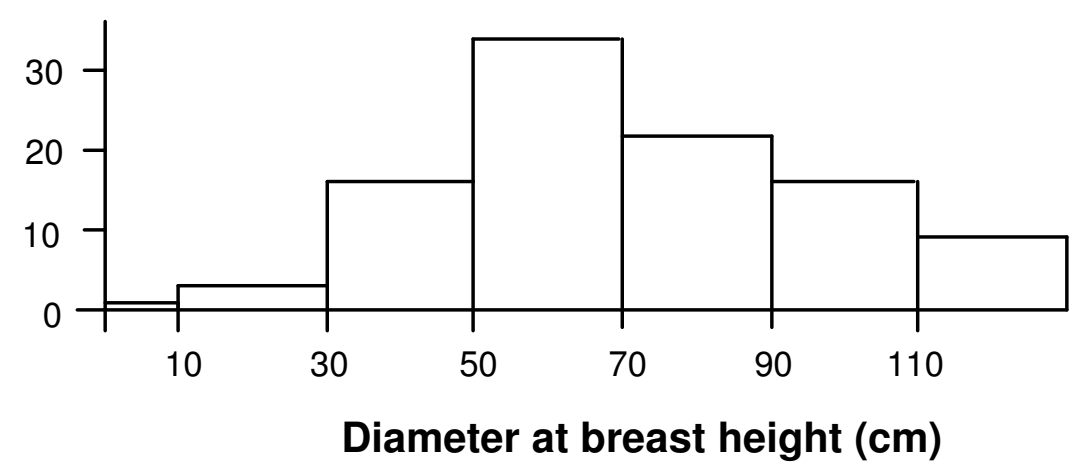

Figure 9.6: $\quad$ Use of different tree sizes in summer and winter.

\subsubsection{Selective tree use}

Because of the small number of observations of yellowhead habitat use made in the winter only the summer observations were used in analysis of selective tree use.

\subsubsection{Variable area plot measures of tree abundance}

Table 9.3 shows the distribution of observations amongst all species and size classes and the results of goodness of fit tests. The frequency of observation of yellowheads in trees is not well predicted by any of the variable area plot measures of abundance. This suggests that yellowheads are selective in their use of trees. 
Table 9.3

The distribution of observations amongst species and size classes of tree

\begin{tabular}{|c|c|c|c|c|c|c|}
\hline Species & Size class & Observed & $\begin{array}{c}\text { basal } \\
\text { area } \\
\end{array}$ & $\begin{array}{l}\text { pected or } \\
\text { stem } \\
\text { density } \\
\end{array}$ & $\begin{array}{l}\text { he basis } \\
\text { surface } \\
\text { area } \\
\end{array}$ & volume \\
\hline \multirow[t]{7}{*}{ Silver beech } & $<10$ & 17 & 19.5 & 682.1 & 64.0 & 5.7 \\
\hline & $10-30$ & 140 & 69.5 & 82.6 & 128.3 & 41.1 \\
\hline & $30-50$ & 384 & 312.2 & 59.1 & 454.0 & 322.0 \\
\hline & $50-70$ & 644 & 450.9 & 37.7 & 487.7 & 518.8 \\
\hline & $70-90$ & 422 & 227.7 & 10.6 & 183.4 & 261.3 \\
\hline & $90-110$ & 168 & 72.5 & 2.1 & 46.9 & 83.2 \\
\hline & $110+$ & 52 & 47.8 & 0.9 & 24.0 & 52.7 \\
\hline \multirow[t]{7}{*}{ Red beech } & $<10$ & 10 & 19.5 & 689.7 & 70.2 & 5.7 \\
\hline & $10-30$ & 54 & 76.9 & 92.1 & 184.1 & 58.7 \\
\hline & $30-50$ & 34 & 76.8 & 17.8 & 117.7 & 77.3 \\
\hline & $50-70$ & 57 & 90.7 & 7.7 & 110.7 & 117.5 \\
\hline & $70-90$ & 125 & 115.4 & 5.4 & 122.1 & 172.5 \\
\hline & $90-110$ & 173 & 124.1 & 3.6 & 89.5 & 161.1 \\
\hline & $110+$ & 197 & 292.5 & 4.5 & 200.2 & 495.4 \\
\hline \multirow[t]{5}{*}{ Mountain beech } & $<10$ & 1 & 1.6 & 23.6 & 5.3 & 0.6 \\
\hline & $10-30$ & 8 & 15.4 & 12.8 & 39.3 & 15.1 \\
\hline & $30-50$ & 11 & 8.1 & 1.6 & 15.0 & 10.4 \\
\hline & $50-70$ & 17 & 25.6 & 2.1 & 31.0 & 33.5 \\
\hline & $70+$ & 14 & 0.7 & 0.0 & 0.6 & 0.8 \\
\hline \multirow[t]{7}{*}{ Dead trees } & $<10$ & 0 & 4.9 & 125.0 & 10.3 & 0.8 \\
\hline & $10-30$ & 0 & 19.6 & 31.8 & 19.0 & 4.9 \\
\hline & $30-50$ & 5 & 89.4 & 17.5 & 39.9 & 27.9 \\
\hline & $50-70$ & 5 & 146.7 & 12.1 & 46.2 & 49.4 \\
\hline & $70-90$ & 5 & 104.6 & 4.7 & 44.2 & 64.0 \\
\hline & $90-110$ & 2 & 40.0 & 1.2 & 4.7 & 8.0 \\
\hline & $110+$ & 0 & 117.8 & 1.9 & 4.3 & 10.6 \\
\hline \multirow[t]{5}{*}{ Other trees } & $<10$ & 26 & 15.9 & 651.3 & 40.5 & 2.9 \\
\hline & $10-30$ & 26 & 22.7 & 38.3 & 27.3 & 7.4 \\
\hline & $30-50$ & 1 & 4.2 & 0.7 & 1.7 & 1.2 \\
\hline & $50-70$ & 1 & 4.4 & 0.3 & 5.3 & 5.8 \\
\hline & $70+$ & 22 & 3.5 & 0.2 & 3.7 & 4.7 \\
\hline \multirow{3}{*}{\multicolumn{2}{|c|}{$\begin{array}{l}\chi^{2} \\
\text { Degrees of freedom } \\
\text { Probability }\end{array}$}} & & 1412 & 72434 & 1639 & 1399 \\
\hline & & & 30 & 30 & 30 & 30 \\
\hline & & & 0.0001 & 0.0001 & 0.0001 & 0.0001 \\
\hline
\end{tabular}

To determine which species and size classes yellowheads favour, separate analysis by species and size class were undertaken (Tables 9.4 and 9.5). 
Table 9.4

The distribution of observations amongst tree species

\begin{tabular}{l||r|rrrr} 
& & \multicolumn{4}{|c}{ Expected on the basis of } \\
Species & Observed & $\begin{array}{r}\text { basal } \\
\text { area }\end{array}$ & $\begin{array}{c}\text { stem } \\
\text { density }\end{array}$ & $\begin{array}{c}\text { surface } \\
\text { area }\end{array}$ & volume \\
\hline & & & & & \\
Silver beech & 1827 & 1200 & 875 & 1388 & 1285 \\
Red beech & 650 & 796 & 821 & 894 & 1088 \\
Mountain beech & 51 & 51 & 40 & 91 & 60 \\
Dead trees & 17 & 523 & 194 & 168 & 166 \\
Other trees & 76 & 51 & 691 & 79 & 22 \\
& & & & & \\
$\chi^{2}$ & & 856 & 1783 & 359 & 671 \\
Degrees of freedom & & 4 & 4 & 4 & 4 \\
Probability & & 0.0001 & 0.0001 & 0.0001 & 0.0001
\end{tabular}

Table 9.5

The distribution of observations amongst size classes of tree

\begin{tabular}{|c|c|c|c|c|c|}
\hline Size class $(\mathrm{cm})$ & Observed & $\begin{array}{l}\mathrm{E} x \\
\text { basal } \\
\text { area } \\
\end{array}$ & $\begin{array}{l}\text { ected on } \\
\text { stem } \\
\text { density }\end{array}$ & $\begin{array}{c}\text { basis of } \\
\text { surface } \\
\text { area }\end{array}$ & volume \\
\hline$<10$ & 54 & 61 & 2172 & 190 & 16 \\
\hline $10-30$ & 228 & 204.1 & 257.6 & 398 & 127.2 \\
\hline $30-50$ & 435 & 490.7 & 96.7 & 628.3 & 438.8 \\
\hline $50-70$ & 724 & 718.3 & 59.9 & 680.9 & 725 \\
\hline $70-90$ & 588 & 451.9 & 20.9 & 354 & 503.3 \\
\hline $90-110$ & 343 & 236.6 & 6.9 & 141.1 & 252.3 \\
\hline $110+$ & 249 & 458.1 & 7.3 & 228.5 & 558.7 \\
\hline$\chi^{2}$ & & 194 & 50376 & 678 & 392 \\
\hline Degrees of freedom & & 6 & 6 & 6 & 6 \\
\hline Probability & & 0.0001 & 0.0001 & 0.0001 & 0.0001 \\
\hline
\end{tabular}

Yellowheads do not appear to use tree species in proportion to their abundance, regardless of which measure of tree abundance is used. On the basis of stem density, yellowheads appear to use silver beech, dead trees and other trees less than expected and red beech more. However, stem density is not a good measure of tree abundance 
since it takes no account of varying tree size. The other three measures, all of which take account of tree size, indicate that yellowheads use silver beech more than expected and red beech and dead trees less; there is no clear pattern for mountain beech and other trees

Regardless of which measure of abundance was used, significant selectivity of tree size was apparent, but there was no clear pattern of size class selectivity amongst the three measures that take tree size into account.

Variable area plots indicate that yellowheads selectively use silver beech, but provide no unequivocal evidence of selection of trees on the basis of size.

\subsubsection{Phiangle measures of tree abundance}

Phiangle revealed the same pattern of tree species preference with yellowheads preferring silver beech and avoiding all other species (Table 9.6).

\section{Table 9.6}

The distribution of observations amongst species of tree and goodness of fit test of hypotheses that yellowheads use tree species in proportion to their surface areas as measured using phiangle

\begin{tabular}{|c|c|c|}
\hline Species & Observed & Expected \\
\hline Silver beech & 446 & 271.6 \\
\hline Red beech & 319 & 478.2 \\
\hline Mountain beech & 0 & 0.1 \\
\hline Dead trees & 1 & 6.8 \\
\hline Other trees & 8 & 17.3 \\
\hline & $=175$ & \\
\hline Degrees of freedom & $=4$ & \\
\hline Probability & 0.0001 & \\
\hline
\end{tabular}

Like variable area plots, phiangle indicated that yellowheads had strong preferences for some tree sizes. Unlike the variable area plot analysis however, there was a clear pattern of preference for large trees and avoidance of small ones (Table 9.7). 


\section{Table 9.7}

The distribution of observations amongst size classes of tree and goodness of fit test of hypotheses that yellowheads use size classes in the proportion to their surface areas as measured using phiangle

\begin{tabular}{|c|c|c|}
\hline $\begin{array}{c}\text { Tree size } \\
(\mathrm{DBH} \text { in } \mathrm{cm})\end{array}$ & Observed & Expected \\
\hline$<10$ & 7 & 51.1 \\
\hline $10-30$ & 57 & 86.2 \\
\hline $30-50$ & 56 & 75.5 \\
\hline $50-70$ & 153 & 168.3 \\
\hline $70-90$ & 207 & 204.0 \\
\hline $90-110$ & 183 & 165.5 \\
\hline $110+$ & 111 & 23.5 \\
\hline$\chi^{2}$ & $=383$ & \\
\hline Degrees of freedom & $=6$ & \\
\hline Probability & 0.0001 & \\
\hline
\end{tabular}

In variable area plots, trees are partitioned amongst species and size classes, but in phiangle, surface area of trees can be partitioned according to any classification. Thus it is possible to determine whether yellowheads use perch sites or height strata in proportion to their surface areas (Tables 9.8 and 9.9). Yellowheads apparently prefer small branches to all other perching sites, and they show preferences for some height strata over others. When all tree species are lumped together (as in Table 9.9) yellowheads show no clear pattern of height strata preference. However, when the same analysis is carried out on a species by species basis for the two main species (Table 9.10), the pattern is clear: Yellowheads prefer the top $15 \mathrm{~m}$ of silver beech and the top $10 \mathrm{~m}$ of red beech. 


\section{Table 9.8}

The distribution of observations amongst perch sites and goodness of fit test of hypotheses that yellowheads use perch sites in proportion to their surface areas as measured using phiangle

\begin{tabular}{l|cc} 
Perch site & Observed & Expected \\
\hline & & \\
Twigs and foliage & 250 & 387.2 \\
Small branches & 177 & 16.1 \\
Large branches & 37 & 49.2 \\
Trunks & 24 & 35.5
\end{tabular}

$$
\begin{array}{rlc}
\chi^{2} & = & 1665 \\
\text { Degrees of freedom } & = & 3 \\
\text { Probability } & = & 0.0001
\end{array}
$$

Table 9.9

The distribution of observations amongst height strata and goodness of fit test of

\begin{tabular}{|c|c|c|}
\hline Height strata (m) & Observed & Expected \\
\hline $0-5$ & 40 & 917 \\
\hline $5-10$ & 73 & 151.1 \\
\hline $10-15$ & 207 & 171.3 \\
\hline $15-20$ & 177 & 146.2 \\
\hline $20-25$ & 108 & 145.9 \\
\hline $25-30$ & 123 & 47.2 \\
\hline $30+$ & 42 & 16.5 \\
\hline$\chi^{2}$ & \multicolumn{2}{|c|}{254} \\
\hline Degrees of freedom & \multicolumn{2}{|c|}{6} \\
\hline Probability & \multicolumn{2}{|c|}{0.0001} \\
\hline
\end{tabular}
hypotheses that yellowheads use height strata in proportion to their surface areas as measured using phiangle 
Table 9.10

The distribution of observations amongst height strata and goodness of fit test of hypotheses that yellowheads use height strata in proportion to their surface areas as measured using phiangle in red and silver beech trees

\begin{tabular}{|c|c|c|c|c|}
\hline \multirow{2}{*}{$\begin{array}{l}\text { Height } \\
\text { (m) }\end{array}$} & \multicolumn{2}{|c|}{ Red beech } & \multicolumn{2}{|c|}{ Silver beech } \\
\hline & Observed & Expected & Observed & Expected \\
\hline $0-5$ & 28 & 69.3 & 9 & 24.4 \\
\hline $5-10$ & 54 & 115.3 & 16 & 46.2 \\
\hline $10-15$ & 161 & 125.5 & 45 & 60.9 \\
\hline $15-20$ & 123 & 83.2 & 51 & 62.4 \\
\hline $20-25$ & 79 & 51.6 & 52 & 74.6 \\
\hline $25-30$ & - & - & 95 & 30.8 \\
\hline $30+$ & - & - & 42 & 10.8 \\
\hline$\chi^{2}$ & & & & \\
\hline $\mathrm{df}$ & & & & \\
\hline $\mathrm{p}$ & & & & \\
\hline
\end{tabular}

\section{4}

\section{DISCUSSION}

\subsubsection{Some problems with the habitat use sampling method}

\subsubsection{Pooling and repeated sampling bias}

Morrison (1984), Bradley (1985), and Machlis et al. (1985) have demonstrated that repeated sampling of the same bird can lead to biased samples of their behaviour. If individual birds differ in their behaviour, then bias will result if all birds are not sampled equally. Also, if birds tend to change from one activity to another infrequently, consecutive samples will not be independent of one another.

Airola and Barret (1985) overcame these problems by "... regarding each individual as an observation when determining frequencies and sample sizes for statistical test. Thus, when $n$ consecutive observations of an individual were recorded, each observation contributed to the species' total frequency by a value of $1 / n$ and all observations of the individual contributed $\Sigma^{1} /{ }_{n}=1$ to the species' frequencies."

Such a solution was not possible in my study since birds were not always individually identifiable, and more than one bird was often observed in a single series of consecutive observations. Morrison (1984) suggested that the problems of bias can be overcome by including a large sample of observations from numerous individuals in any analysis. He 
suggests a minimum of 30 individuals and 150 observations but the impossibility of identifying all yellowheads means I cannot assess my data using these guide-lines.

\subsubsection{Visibility bias}

Bradley (1985) said that estimates of the proportion of time birds spend in arbitrarily defined activity states, may be biased by differences in observability of these activity states. The same argument can be extended to estimates of the proportion of time birds spend in various tree types. For example, yellowheads calling in trees with little foliage may be recorded more frequently than yellowheads foraging quietly in trees with dense foliage. Wagner (1981) identified two sources of visibility bias, discovery and loss bias. Discovery bias results from differences in the ease with which birds in different activity states can be found. Loss bias results from differences in the chance that a bird will be lost during a bout of recording.

The "count all" (i.e., include all observations in an analysis, Bradley 1985) strategy that I have adopted avoids discovery bias by overwhelming the potentially biased early observations with far more numerous subsequent observations (Bradley 1985). There remains a considerable potential for loss bias in my data since it became obvious that some trees and activity types frequently result in my losing the bird. When birds flew, they were frequently lost to sight, as were birds that foraged in the tops of very tall red beech trees.

\subsubsection{Assessing the magnitude of bias}

To investigate possible bias resulting from the lack of independence of consecutive observations and variable visibility, I compared the distribution of observations amongst activities and tree species, using "count-all" and "count first" (Bradley 1985) sampling strategies. In "count-all" sampling observations may not be independent, but discovery bias is unlikely. In "count-first" sampling, only the first observation from each series is included; observations are independent, loss bias non-existent, but the possibility of discovery bias is high. I found no association between distribution amongst trees or activities, and sampling strategy (Tables 9.11 and 9.12), and conclude that neither pooling nor visibility bias is significant in my data. 
Table 9.11

Test for the effect of sampling regime on the distribution of observations amongst activities

\begin{tabular}{l|cc} 
Activity & \multicolumn{2}{|c}{ Sampling regime } \\
& Count all & Count first \\
\hline Scan & 741 & 45 \\
Glean & 531 & 34 \\
Rip & 234 & 10 \\
Other & 285 & 10
\end{tabular}

$$
\begin{aligned}
& \chi^{2}=3.736 \\
& \mathrm{df}=3 \\
& \mathrm{p}=0.291
\end{aligned}
$$

\section{Table 9.12}

\begin{tabular}{|c|c|c|c|}
\hline \multirow[t]{2}{*}{ Species } & \multirow{2}{*}{$\begin{array}{l}\text { Size class } \\
(\mathrm{cm})\end{array}$} & \multicolumn{2}{|c|}{ Sampling regime } \\
\hline & & Count all & Count first \\
\hline \multirow{6}{*}{ Silver beech } & $0-30$ & 157 & 7 \\
\hline & $30-50$ & 384 & 27 \\
\hline & $50-70$ & 644 & 27 \\
\hline & $70-90$ & 422 & 15 \\
\hline & $90-110$ & 168 & 7 \\
\hline & $110+$ & 52 & 1 \\
\hline \multirow[t]{7}{*}{ Red beech } & $<10$ & 10 & 1 \\
\hline & $10-30$ & 54 & 4 \\
\hline & $30-50$ & 34 & 1 \\
\hline & $50-70$ & 57 & 7 \\
\hline & 70-90 & 125 & 8 \\
\hline & $90-110$ & 173 & 12 \\
\hline & $110+$ & 197 & 7 \\
\hline \multirow[t]{2}{*}{ Mountain beech } & $0-50$ & 20 & 1 \\
\hline & $50+$ & 31 & 2 \\
\hline Dead trees & $0+$ & 17 & 1 \\
\hline Others & $0+$ & 76 & 4 \\
\hline & $\chi^{2}$ & $=15.964$ & \\
\hline Degrees & of freedom & $=16$ & \\
\hline & Probability & $=0.455$ & \\
\hline
\end{tabular}

Test for the effect of sampling regime on the distribution of observations amongst tree species and size classes 
Though Machlis et al. (1985) criticised studies using sampling regimes such as mine, they noted that in field studies inability to recognise birds often prevents measurement and elimination of bias. They did not suggest that such studies were valueless; only that the results should be viewed with caution. My comparison of "count-all" and "countfirst" sampling strategies has shown that although "count-all" sampling is potentially biased, in this case any bias is insignificant. The analyses presented in this chapter can be viewed with confidence.

In any case for practical reasons "count all" sampling was the only practical strategy. Yellowheads spend most of their time high in trees, where it is difficult to locate them, but once found they can often be followed for long periods of time. Making only a few, and always the same number of observations for each bird would have overcome some potential biases, but it would have been much more time consuming and could only have been used on recognisable birds (i.e., banded ones). Since habitat use sampling was only a small part of this study, it did not justify any increase in effort.

Read (1988a) attempted to overcome potential bias by using only every fifteenth observation in his data set, but did not determine whether or not this made any difference to his conclusions. He may have unnecessarily thrown away most of his data.

\subsubsection{Foraging behaviour}

The patterns of activity and use of perching sites revealed in this study are very similar to those of yellowheads near Arthur's Pass (Read 1988a and 1988b). In summer, yellowheads spent $90 \%$ of their time foraging and this proportion increased in the winter to $95 \%$. At the same time there was a transition from gleaning on twigs and foliage to ripping and probing on trunks and branches. Read (1988a) suggested that as yellowheads are largely insectivorous, the seasonal changes in foraging behaviour may reflect seasonal changes in the abundance of their prey. The high incidence of foliage gleaning in summer may reflect the high numbers of caterpillars present on leaves at that time. In winter the number of caterpillars drops and yellowheads may shift to eating overwintering larvae and pupae in the bark. They spend a greater proportion of their time foraging because days are shorter and food less abundant.

Decreased use of small trees in winter also probably resulted from the shift from foliage to bark gleaning, as small trees have proportionately less bark than large ones (Read 1988a). Similarly decreased use of red beech in the winter resulted from the fact that the average size of red beech trees at Knobs Flat was smaller than silver beech. Birds shifting from small to large trees will tend to move from red to silver beech. Moving from red to silver beech in the winter because silver beech trees were larger may not 
occur in other forests. At Knobs Flat the largest trees are red beech, but the average size of red beech is small because there are large number of small red beeches growing in areas of recent windthrow. In most beech forests red beech is likely to be larger on average than silver beech.

\subsubsection{Selective tree use}

I found that using stem density as a measure of tree availability when assessing selective tree use, is misleading. The largest trees inevitably appear to be selectively used, since they make up most of the forest biomass. Measures of tree size derived from variable area plots indicated that yellowheads preferred silver beech to red, but they revealed no pattern in tree size selectively. Phiangle surface area measures also showed that yellowheads preferred silver beech, and they revealed a pattern of tree size selectivity, i.e., yellowheads preferred the largest trees. Phiangle also showed that yellowheads spent more time on small branches and in the tops of trees than would be predicted on the basis of their surface areas.

Read (1988a) also found that yellowheads preferred large trees and he suggested that ". . the time yellowheads spend on a tree is related to its size." In fact yellowheads appear to use large trees even more often than one would predict on the basis of their size. Read's finding that yellowheads preferred red beech is entirely an artifact of his use of stem density as a measure of tree abundance.

Read (1988a) suggested "Larger trees might support more prey, either because they have larger surface areas (and therefore prey are more concentrated for a given volume of forest), or because they contain a greater density and diversity of invertebrates per unit surface area, perhaps because they offer a greater variety of habitats to invertebrates (e.g., more furrows in the bark and more attached moss and lichen)." A similar explanation might exist for their apparent preference for silver beech. The bark of red beech is loose and is constantly shed, so that red beech supports few epiphytic mosses, ferns and lichens. In contrast, silver beech bark is firm and supports abundant mosses, ferns and lichens amongst which yellowheads often forage for invertebrates.

In Chapter 8 I showed that yellowheads preferred forests with large trees and a large red beech component, and in this chapter I have attempted to determine whether or not these forest preferences result from preferential use of large trees and red beech trees. Yellowheads do preferentially use large trees, but they do not preferentially use red beech. I suspect that the apparent preference for red beech dominated forests, results from the fact that red beech grows on fertile sites (Wardle 1984) and forests with large red beech components typically comprise not only very large red beech trees but very large trees of every species. 
This study suggests that yellowheads prefer forests of large trees not because such forests grow on the most productive sites, but because even within forests on poor sites they prefer to forage on the largest trees. This conclusion is corroborated by Spurr's (1986) study in which he found that yellowheads did not return to logged forests for more than 25 years after they were logged. Had site characteristics determined whether or not yellowheads lived in the forests Spurr studied, then yellowheads should have recolonised the young forest that regenerated after logging.

It is clear from this study that the yellowhead's over-riding preference is for large trees, rather than for any species of tree or for any suite of site characteristics- they are tall forest specialists. 


\section{CHAPTER 10}

\section{NEST SITE SELECTION BY YELLOWHEADS}

10.1 INTRODUCTION

At the beginning of this thesis I proposed 4 questions about yellowhead nest site selection that I hoped to answer:

1. Does hole nesting protect yellowheads from predation and parasitism by longtailed cuckoos?

2. Is there evidenece that hole nesting is a recently acquired trait? Well developed patterns of nest site selection might indicate that it is not.

3. Do yellowhead patterns of habitat preference result from selective use of some types of tree for nesting?

4. Does hole nesting in yellowheads match the normal explanations for the evolution of hole nesting?

The first question was answered in Chapter 7. This chapter aims to answer the remaining three.

\subsubsection{Nest site selection}

Yellowheads nest in holes in trees, whereas their nearest relatives, the brown creeper and whitehead, nest in open nests, as do their likely next-nearest relatives, the Australian pachycephalines (Frith 1976). Soper (1976) suggested that hole nesting was a recently acquired trait. If hole nesting is a recently acquired trait then nest site selection may not be well developed. In this chapter I investigate nest site selection in yellowheads.

\subsubsection{Nest sites and habitat preference}

Elsewhere (Chapter 8 and 9) I have shown that the distribution of yellowheads during their breeding season is related to both forest structure and composition; specifically, yellowheads prefer forests with a few large trees. Habitat preferences result from selective use of a variety of features of which nest sites is but one possibility. One of the main aims of this Chapter is to determine whether or not selection or availability of nest sites has any effect on the patterns of habitat preference of yellowheads. Specifically I aim to:

1. determine whether yellowheads nest more frequently in some types of tree than others. 
2. determine whether the patterns of use of different tree species for nesting can help explain the observed patterns of habitat preference.

\subsubsection{The evolution of hole nesting in yellowheads}

Cavity nesting is a frequently studied phenomenon which has often been assumed to be a mechanism for reducing nest predation (e.g., Alerstam \& Högstedt 1981, von Haartman 1957, Lack 1968). Given that hole nesting is advantageous, only competition for holes prevents all species from doing it (Lack 1968, Alerstam and Högstedt 1981), and Alerstam and Högstedt suggest that the "choice" of nesting in cavities is a trade off between reduced predation and the reduced availability of nest sites compared with nesting in the open.

This model is not without its detractors. Nilsson (1986) showed that nests in holes did not necessarily suffer low rates of predation, and he suggested that thermoregulation and nest parasitism, as well as competition and predation can influence the evolution of hole nesting. Alerstam and Högstedt (1981) themselves suggested cavity nesting would only be advantageous when the most important nest predators detected their prey by sight (e.g. avian predators). If the main nest predators were mammals or reptiles that detected their prey by smell and sound, Alerstam and Högstedt believed cavity nesting would offer little or no advantage over nesting in the open. They further suggested that where mammal or reptile predation was common, cavity nesting could be counterproductive since it would expose the adults, as well as the clutch, to the risk of predation.

The evolution of hole nesting in yellowheads seems to fit Alerstam and Högstedt's (1981) model quite well. In pre-human New Zealand there were no mammalian predators and the main predators of yellowhead nests would have been long-tailed cuckoos, falcons, harriers, moreporks and laughing owls. Building nests in holes would have made them more difficult for these predators to find and could even have protected the nests once found if the entrance holes were small enough (Chapter 7). As long as plenty of suitable holes were available, nesting in holes would have been advantageous. Unfortunately in modern New Zealand yellowheads are exposed to mammalian predators from which hole nesting provides no protection.

To determine whether hole nesting in yellowheads fits the widely accepted model for the evolution of such behaviour, this study aims to:

1. determine to what extent yellowhead nest hole entrances might exclude native predators.

2. estimate the abundance of nest sites.

3. assess competition for such sites. 
The first point has been well covered in Chapter 7, the second and third are addressed here.

\section{2}

METHODS

\subsubsection{Nests}

All the nests of about 13 pairs of yellowheads that nested at Knobs Flat were found during the four breeding seasons between 1984 and 1988. Nests were found using the methods outlined in Chapter 5. Every time a nest was found the following were recorded:

1. The species of tree that the nest was in.

2. The height of the tree that the nest was in.

3. The diameter at breast height of the tree the nest was in.

4. The position of the nest in grid co-ordinates.

Where possible the tree was climbed and further information on the nest site collected, including:

5. The height of the nest above the ground.

6. The diameter of the branch or trunk that the nest was in.

7. The largest dimension of the entrance hole.

8. The smallest dimension of the entrance hole.

9. The distance between the bottom lip of the entrance hole and the bottom of the cavity that the nest was in.

10. The direction the nest faced (compass bearing).

Once found nests were monitored regularly to:

11. Determine whether or not they successfully produced chicks.

12. Determine the reason for nest failure.

13. Determine whether or not they were parasitised by long-tailed cuckoos.

Each nest site was marked so that nest site re-use could be detected.

\subsubsection{Vegetation measurement}

Methods of vegetation measurement are described in the previous chapter (section 9.2.2) 


\section{RESULTS}

\subsubsection{Description of nests}

Ninety five nests were found within the study area in 81 sites (some sites were used more than once).

All yellowhead nests were in holes in trees, and all but one consisted of loosely woven bowl of mosses, filmy ferns, lichens and a few feathers placed in the bottom of the hole. The one exception was a nest in which the eggs were laid directly on powdered rotten wood in the bottom of the hole.

Eighty two percent of nests were built in knot holes; the remainder were built in holes where rotten wood had crumbled away leaving a cavity.

The maximum dimension of the entrance hole ranged from 4 to $50 \mathrm{~cm}$ (mean=10.7, $\mathrm{n}=74)$, and the minimum dimension from 2.5 to $15 \mathrm{~cm}$ (mean=4.7, $\mathrm{n}=74)$. The smallest hole was $4 \infty 3 \mathrm{~cm}$, and the largest was $15 \infty 16 \mathrm{~cm}$. Hole depth ranged from 0 to $30 \mathrm{~cm}$ (mean=11.2, $\mathrm{n}=74$ ). The inside dimensions of the cavities were difficult to measure but appeared to be not less than about $10 \mathrm{~cm}$ in cross section.

All nests were in beech trees. Forty four percent were in live silver beech trees, $36 \%$ in live red beech, $4 \%$ in live mountain beech, and $16 \%$ in standing dead trees.

Though only $16 \%$ of nests were in dead trees, a higher proportion, $27 \%$, were in dead wood.

Most nests (67\%) were in the trunks of trees, but $30 \%$ were in large trunk-like branches.

Nests were between 4 and $30 \mathrm{~m}$ off the ground (mean=13.38, std=7.59, $\mathrm{n}=92$ ).

Nests were predominantly in large old trees; the average height of nest trees was $22.76 \mathrm{~m}(\mathrm{SD}=7.12, \mathrm{n}=9)$, and the average $\mathrm{DBH} 83.81 \mathrm{~cm}(\mathrm{SD}=33.96, \mathrm{n}=95)$. No nests were in trees less than $30 \mathrm{~cm} \mathrm{DBH}$ or in branches or trunks less than $20 \mathrm{~cm}$ in diameter.

Only two of the 81 nest sites were used for consecutive nesting attempts in the same breeding season, but 11 sites were used in two breeding seasons, and one in three.

\subsubsection{Successful and unsuccessful nest sites}

To test whether some nest sites might be more prone to failure than others, nests were classified as successful if they fledged chicks and unsuccessful if they did not. 
Association between success and tree species was tested in a contingency table (Table 10.1) and the continuous parameters of successful and failed nests were compared using the Mann-Whitney test (Sokal \& Rohlf 1981) (Table 10.2). To test for association between nest success and the direction the nest faces, the directions that nests were faced were classified into four groups, north, south, east and west (Table 10.3).

\section{Table 10.1}

Contingency table analysis for association between nest success and tree species.

\begin{tabular}{l|ccr} 
Tree species & \%success & \%failure & n \\
\hline & & & \\
Red beech & 43 & 57 & 30 \\
Silver beech & 52 & 48 & 42 \\
Dead tree & 60 & 40 & 15 \\
Other & 0 & 100 & 4
\end{tabular}

$$
\chi^{2}=5.135
$$

Degrees of freedom $=3$

Probability $=0.16$

\section{Table 10.2}

A comparison of the descriptive parameters of successful and failed nests and MannWhitney tests for difference.

\begin{tabular}{l|rccc} 
& \multicolumn{3}{|c}{ Mean } & \\
Parameter & successful & failed & $\mathrm{Z}$ & Probability \\
Height of nest & 13.774 & 12.853 & 0.380 & 0.70 \\
Height of tree & 22.626 & 22.409 & 0.053 & 0.96 \\
DBH & 82.809 & 83.409 & 0.117 & 0.91 \\
DNH & 47.826 & 41.791 & 0.919 & 0.36 \\
Max. hole dimension & 10.887 & 10.734 & 1.008 & 0.31 \\
Min. hole dimension & 4.825 & 4.578 & 0.134 & 0.89 \\
Hole depth & 12.659 & 9.29 & 1.810 & 0.07
\end{tabular}




\section{Table 10.3}

Contingency table analysis for association between nest success and the directions that nests faced.

\begin{tabular}{l|ccc} 
Direction & \%success & \%failure & n \\
\hline & & & \\
North & 45 & 55 & 20 \\
East & 44 & 56 & 16 \\
South & 58 & 42 & 19 \\
west & 61 & 39 & 31
\end{tabular}

$$
\begin{aligned}
\chi^{2} & =2.096 \\
\text { Degrees of freedom } & =3 \\
\text { Probability } & =0.55
\end{aligned}
$$

There were no significant differences between successful and failed nests, though successful nests were nearly significantly deeper than unsuccessful ones. Perhaps deep nests are less prone to predation.

\subsubsection{Nest site selectivity}

If birds are selective in their use of nest sites then at least one of the following will occur:

1. repeated use of nest sites that have been successful. Some birds tend to re-use nests in which they have successfully raised chicks in the past, and avoid reusing those in which they failed (e.g., eastern bluebirds (Sialia sialis) (Herlugson 1983), great tits (Parus major) (Harvey et al. 1979)).

2. repeated avoidance of nest sites that have been used before. Some species avoid re-using nests as a predator avoidance mechanism (e.g. Tengmalm's Owl (Aegolius funereus) (Sonerud 1985)) or possibly to avoid the build up of nest parasites.

3. preferential use of some sites regardless of their history. Some species find some sites inherently preferable because they are easy to find, offer better protection from predators or offer better protection from the elements (e.g., Nilsson 1984, Belles-Ilses and Picman 1986, Lumsden 1986).

I examined my data on yellowhead nest site use for evidence of any of the above.

\subsubsection{Re-use and knowledge of a nest site}

To test whether birds that had already used a nest were more or less likely to re-use it than other birds, I examined the history of use of every nest site. Once a nest had been 
used (and I had found it) every subsequent breeding season was regarded as an opportunity for the nest to be re-used. Each of these opportunities was then crossclassified according to:

1. whether or not the nest was re-used.

2. whether or not the birds that occupied the territory in which the nest site occurred, knew of the site. A nest site was regarded as being "known of" when at least one of the birds that had previously used the nest was still resident in the territory. A nest site was regarded as being "unknown" when birds with no known experience of the nest site occupied the area around the nest site.

Nest sites were no more likely to be re-used when the territory holders knew of the site than when they did not (Table 10.4).

\section{Table 10.4}

Contingency table testing for association between "knowledge" of a nest site and its reuse.

\begin{tabular}{l|cc} 
& Known & Unknown \\
\hline & & \\
Re-used & 5 & 76
\end{tabular}

Degrees of freedom $=1$

$$
\chi^{2}=1.752
$$

Probability $=0.19$

\subsubsection{Re-use of successful nests}

To test whether birds were more likely to use sites in which they had successfully raised chicks than sites which they had not, each nesting attempt was classified according to whether it was a success or a failure and whether or not the site was subsequently reused (Table 10.5).

Only the nesting attempts of pairs of birds that occupied the same territory in consecutive years were used in the analysis, since the previous success or failure of a site was probably only known to the birds that had used it. Nest sites first used in the last year of my study could not be included because I knew nothing of their subsequent history of use. 


\section{Table 10.5}

Contingency table testing for association between nest success and re-use.

\begin{tabular}{l|cc} 
& Chicks & No chicks \\
\hline & 10 & 4 \\
Re-used & 32 & 31
\end{tabular}

$$
\begin{aligned}
\chi^{2} & =1.967 \\
\text { Degrees of freedom } & =1 \\
\text { Probability } & =0.16
\end{aligned}
$$

The fact that a few yellowheads repeatedly used "failed" nest sites and the lack of statistical association between success and re-use suggests that past success or failure has little influence on nest site choice.

\subsubsection{Inherent preferability of nest sites}

Comparison of nest holes used with those available is the obvious way of determining whether yellowheads prefer some holes to others. Measuring availability of nest sites has been attempted (van Balen et al. 1982) but only low nests were included. The difficulty and time it would take to inspect all available nest sites makes this approach impractical for yellowheads.

Another way to detect nest site selectivity is from the pattern of re-use of sites. Nonrandom repeated use of the same sites indicates that yellowheads prefer some sites to others. Since I have shown that the history of use of sites has no effect on their re-use, any non-random re-use of sites indicates that yellowheads choose sites on the basis of characteristics other than their history.

Testing for non-random re-use of nest sites is analogous to testing for equal catchability in mark-recapture studies. In mark-recapture terms, a breeding season becomes a trapping session, nest sites become the animals, and the use of a nest site by a yellowhead becomes a capture. Recaptures occur when a nest site is used more than once. If nests are used at random then the frequency of single and multiple uses should have a zero-truncated binomial distribution, which is approximated by the more tractable zero-truncated Poisson distribution. Thus we can test for non-random use by comparing the frequency distribution of single and multiple uses with a zero-truncated Poisson distribution, and we can assess the significance of any difference using a chisquared goodness of fit test (Caughley 1977) (Table 10.6). 


\section{Table 10.6}

Observed and expected frequencies of single and multiple nest site use and goodness of fit test. Expected frequencies are estimated from a zero truncated Poisson distribution

(Caughley 1977).

\begin{tabular}{|c|c|c|}
\hline No. of times used & No. of sites & Expected frequency \\
\hline 1 & 69 & 69.26 \\
\hline 2 & 11 & 10.58 \\
\hline $3-4$ & 1 & 1.09 \\
\hline Degrees & $\begin{aligned} \chi^{2} & =0.025 \\
\text { lom } & =1 \\
\text { ility } & >0.10\end{aligned}$ & \\
\hline
\end{tabular}

The test indicates that I cannot reject the null hypothesis that nests are used randomly. One should treat this result with some caution as the sample size is small and one of the expected frequencies is less than five. However the fit between the observed and expected is not only close, it is perfect; no other observed frequencies could give a smaller value for chi-squared.

\subsubsection{Nest site - vegetation relationships}

\subsubsection{Vegetation near the nest}

To determine whether yellowheads chose to nest in any particular sorts of vegetation, I compared the vegetation in the samples nearest the nests with all of the vegetation samples, i.e., I compared the vegetation near nests with random vegetation samples. The stem densities of all possible combinations of tree species and size class were compared using the Mann-Whitney test (Table 10.7). None were significantly different and I conclude that the vegetation near nests did not differ from the rest of the vegetation. 
Table 10.7

Comparison of vegetation samples near nest sites with random vegetation samples.

\begin{tabular}{|c|c|c|c|c|c|}
\hline \multicolumn{2}{|c|}{$\begin{array}{l}\text { Stem density } \\
\left(\text { stems } / \mathrm{m}^{2}\right) \text { of }\end{array}$} & $\begin{array}{c}\text { Nest } \\
\text { sample }\end{array}$ & $\begin{array}{c}\text { Random } \\
\text { sample }\end{array}$ & $\mathrm{Z}$ & Probability \\
\hline \multirow[t]{7}{*}{ Silver beech } & $<10$ & 0.1100 & 0.1035 & 0.3725 & 0.7092 \\
\hline & $10-30$ & 0.0165 & 0.0162 & 0.2484 & 0.8042 \\
\hline & $30-50$ & 0.0079 & 0.0090 & 0.3152 & 0.7528 \\
\hline & $50-70$ & 0.0039 & 0.0044 & 0.3669 & 0.7136 \\
\hline & $70-90$ & 0.0014 & 0.0019 & 0.9898 & 0.3224 \\
\hline & $90-110$ & 0.0008 & 0.0005 & 1.4909 & 0.1360 \\
\hline & $110-130$ & 0.0001 & 0.0001 & 0.8263 & 0.4088 \\
\hline \multirow[t]{8}{*}{ Red beech } & $<10$ & 0.0727 & 0.0922 & 1.1340 & 0.2568 \\
\hline & $10-30$ & 0.0081 & 0.0109 & 0.5957 & 0.5510 \\
\hline & $30-50$ & 0.0027 & 0.0027 & 0.0696 & 0.9466 \\
\hline & $50-70$ & 0.0013 & 0.0015 & 0.4807 & 0.6307 \\
\hline & $70-90$ & 0.0015 & 0.0018 & 0.6592 & 0.5098 \\
\hline & $90-110$ & 0.0004 & 0.0005 & 0.2441 & 0.8074 \\
\hline & $110-130$ & 0.0003 & 0.0003 & 0.6773 & 0.4982 \\
\hline & $130-150$ & 0.0002 & 0.0003 & 0.7287 & 0.4662 \\
\hline \multirow[t]{7}{*}{ SDT } & $<10$ & 0.0189 & 0.0192 & 0.0481 & 0.9616 \\
\hline & $10-30$ & 0.0041 & 0.0060 & 1.7484 & 0.0804 \\
\hline & $30-50$ & 0.0019 & 0.0028 & 0.9948 & 0.3196 \\
\hline & $50-70$ & 0.0019 & 0.0015 & 0.7111 & 0.4770 \\
\hline & $70-90$ & 0.0005 & 0.0009 & 1.3781 & 0.1680 \\
\hline & $90-110$ & 0.0001 & 0.0002 & 0.2647 & 0.7910 \\
\hline & $110-130$ & 0.0002 & 0.0002 & 0.7405 & 0.4586 \\
\hline \multirow[t]{5}{*}{ Other } & $<10$ & 0.1194 & 0.1531 & 1.3764 & 0.1686 \\
\hline & $10-30$ & 0.0108 & 0.0102 & 0.2687 & 0.7878 \\
\hline & $30-50$ & 0.0000 & 0.0002 & 0.8105 & 0.4174 \\
\hline & $50-70$ & 0.0002 & 0.0002 & 0.2916 & 0.7702 \\
\hline & $70-90$ & 0.0000 & 0.0000 & 0.1776 & 0.8634 \\
\hline \multicolumn{2}{|l|}{ Silver beech } & 0.1407 & 0.1345 & 0.0602 & 0.9522 \\
\hline \multicolumn{2}{|l|}{ Red beech } & 0.0873 & 0.0819 & 1.2981 & 0.1940 \\
\hline \multicolumn{2}{|l|}{ SDT } & 0.0276 & 0.0309 & 0.8401 & 0.4008 \\
\hline \multicolumn{2}{|l|}{ Other } & 0.1304 & 0.1638 & 1.4409 & 0.1496 \\
\hline \multicolumn{2}{|l|}{$<10$} & 0.3210 & 0.3680 & 1.1505 & 0.2498 \\
\hline \multicolumn{2}{|l|}{$10-30$} & 0.0396 & 0.0433 & 1.1871 & 0.2372 \\
\hline \multicolumn{2}{|l|}{$30-50$} & 0.0126 & 0.0148 & 0.7029 & 0.4822 \\
\hline \multicolumn{2}{|l|}{$50-70$} & 0.0073 & 0.0076 & 0.1250 & 0.9004 \\
\hline \multicolumn{2}{|l|}{ 70-90 } & 0.0034 & 0.0037 & 0.1783 & 0.8578 \\
\hline \multicolumn{2}{|l|}{$90-110$} & 0.0013 & 0.0011 & 0.7968 & 0.4254 \\
\hline \multicolumn{2}{|l|}{$110-130$} & 0.0007 & 0.0005 & 1.0572 & 0.2906 \\
\hline \multicolumn{2}{|l|}{$130-150$} & 0.0002 & 0.0003 & 0.7287 & 0.4662 \\
\hline
\end{tabular}

\subsubsection{Nest sites and trees}

To determine whether some trees were used more frequently for nesting than others, I carried out a goodness of fit test where the expected number of nests in each species and 
size class of tree was calculated from the availability of trees in each species and size class (Table 10.8). The number of nest sites a tree has is likely to be related to the surface area of its trunk, so trunk surface area was used as a measurement of availability.

The surface area of each tree trunk was estimated by assuming it was a cone (Whittaker \& Woodwell 1967) and calculated as:

$$
\text { Surface Area }=\frac{\pi \infty \text { diameter } \infty \text { height }}{2}
$$

Furthermore since holes in trunks less than $30 \mathrm{~cm}$ in diameter have cavities too small to be used by yellowheads for nesting, I calculated the surface area excluding trunks and parts of trunks less than $30 \mathrm{~cm}$ in diameter.

\section{Table 10.8}

The observed and expected occurrence of nests in all tree species and size classes and Chi-squared goodness-of-fit test.

\begin{tabular}{ll|cc} 
Species & $\begin{array}{l}\text { Size } \\
\text { class }\end{array}$ & Observed & Expected \\
Silver beech & $30-50$ & 7 & 16 \\
& $50-70$ & 17 & 17 \\
& $70-90$ & 7 & 6 \\
& $90-110$ & 5 & 4 \\
Red beech & $110+$ & 6 & 1 \\
& $30-50$ & 1 & 5 \\
& $50-70$ & 1 & 8 \\
& $70-90$ & 10 & 16 \\
& $90-110$ & 11 & 5 \\
Dead tree & $110-130$ & 3 & 4 \\
& $130+$ & 2 & 6 \\
& $30-50$ & 9 & 1 \\
& $50-70$ & 3 & 2 \\
& $70-90$ & 0 & 0 \\
Other & $90-110$ & 1 & 0 \\
& $110+$ & 0 & 0 \\
& $30-50$ & 1 & 1 \\
& $50-70$ & 3 & 0 \\
\hline
\end{tabular}

$$
\begin{aligned}
\chi^{2} & =124 \\
\text { Degrees of freedom } & =18 \\
\text { Probability } & =0.0001
\end{aligned}
$$


The distribution of suitable nest sites amongst the various sizes and species of tree is not closely related to the surface area of the tree.

To investigate the distribution of nests amongst trees and tree sizes in more detail the occurrence of nests was examined on a species by species and size class by size class basis (Tables 10.9 and 10.10).

Table 10.9

Observed and expected frequencies of nests in size classes of tree on a species by species basis, and chi-squared goodness-of-fit tests.

\begin{tabular}{|c|c|c|c|c|c|c|c|c|c|c|}
\hline & & & & ize $c$ & & & & & & \\
\hline & & $\begin{array}{c}30- \\
50 \\
\end{array}$ & $\begin{array}{c}50- \\
70 \\
\end{array}$ & $\begin{array}{l}70- \\
90 \\
\end{array}$ & $\begin{array}{l}90- \\
110 \\
\end{array}$ & $\begin{array}{c}110- \\
130\end{array}$ & $130+$ & $\chi^{2}$ & df & $\mathrm{p}$ \\
\hline All species & obs & 10 & 28 & 21 & 17 & 10 & 9 & & & \\
\hline & $\exp$ & 22 & 29 & 24 & 10 & 5 & 6 & 19.52 & 5 & 0.00 \\
\hline Dead tree & obs & 2 & 9 & 3 & 0 & 1 & & & & \\
\hline & $\exp$ & 3 & 6 & 5 & 1 & 0 & & 3.13 & 4 & 0.54 \\
\hline Other & obs & 0 & 1 & 3 & & & & & & \\
\hline & $\exp$ & 1 & 2 & 1 & & & & 14.10 & 2 & 0.00 \\
\hline Red beech & obs & 1 & 1 & 10 & 11 & 3 & 8 & & & \\
\hline & $\exp$ & 4 & 6 & 12 & 4 & 3 & 5 & 20.50 & 5 & 0.00 \\
\hline Silver beech & obs & 7 & 17 & 7 & 5 & 6 & & & & \\
\hline & $\exp$ & 15 & 17 & 5 & 4 & 1 & & 45.01 & 4 & 0.00 \\
\hline
\end{tabular}




\section{Table 10.10}

Observed and expected frequencies of nests in species on a size class by size class basis, and chi-squared goodness-of-fit tests.

Size classes

\begin{tabular}{|c|c|c|c|c|c|c|c|}
\hline & & All & $\begin{array}{c}30 \\
-50 \\
\end{array}$ & $\begin{array}{c}50 \\
-70 \\
\end{array}$ & $\begin{array}{c}70 \\
-90 \\
\end{array}$ & $\begin{array}{c}90 \\
-110 \\
\end{array}$ & $110+$ \\
\hline \multirow[t]{2}{*}{ Dead tree } & obs & 15 & 2 & 9 & 3 & 0 & 1 \\
\hline & $\exp$ & 7 & 1 & 3 & 2 & 0 & 0 \\
\hline \multirow[t]{2}{*}{ Other } & obs & 4 & 0 & 1 & 3 & & \\
\hline & $\exp$ & 1 & 0 & 1 & 1 & & \\
\hline \multirow[t]{2}{*}{ Red beech } & obs & 34 & 1 & 1 & 10 & 11 & 11 \\
\hline & $\exp$ & 44 & 2 & 8 & 14 & 9 & 16 \\
\hline \multirow[t]{2}{*}{ Silver beech } & obs & 42 & 7 & 17 & 7 & 5 & 6 \\
\hline & $\exp$ & 43 & 7 & 17 & 5 & 7 & 1 \\
\hline \multirow{3}{*}{\multicolumn{2}{|c|}{$\begin{array}{r}\chi^{2} \\
\text { Degrees of freedom } \\
\text { Probability }\end{array}$}} & 16.99 & 3.78 & 20.18 & 7.27 & 1.29 & 21.53 \\
\hline & & 3 & 3 & 3 & 3 & 2 & 2 \\
\hline & & 0.00 & 0.29 & 0.00 & 0.060 .5 & 0.00 & \\
\hline
\end{tabular}

Table 10.9 shows that yellowheads nest more frequently in large trees than is predicted on the basis of surface area, but that his pattern is not the same for all tree species. Yellowheads nested equally frequently in all sizes of dead tree, whereas in live trees they nested more frequently in the larger size classes. This probably reflects most (53\%) nests in dead trees being in holes in rotten wood and such holes are equally abundant in all size classes of dead tree, whereas most (90\%) holes in live trees are knot holes which are more abundant in large trees.

Table 10.10 shows that yellowheads nest more frequently than predicted in dead trees and "other" trees, and less frequently in red beech.

The high number of nests in dead trees can be explained by dead and live trees having similar numbers of knot holes, but dead trees have many more holes in rotten wood, so that the total number of nest holes available in dead trees is greater.

The low number of nests in red beech is probably attributable to red beech having a very simple structure compared with the other tree species. Red beeches tend to have long 
straight trunks with few holes in them except near the crown, whereas silver beech has a short trunk and holes are common low in the tree.

\subsubsection{Direction of nest holes}

To determine whether yellowhead nest holes tended to face in any particular direction each hole was classified into one of four direction classes (north, south, east or west) and the distribution amongst these four classes was compared with a uniform distribution using chi-square (Table 10.11). Though there were more nests facing north and west, the difference was not significant. To further test for a direction preference north and west were lumped together as were south and east (Table 10.12). There were significantly more nests facing north and west than there were facing south and east.

\section{Table 10.11}

Nest site direction and a goodness-of-fit test to test for non-random directions: four directions.

\begin{tabular}{l|cc} 
Direction & Observed & Expected \\
\hline North & 27 & 23.25 \\
East & 19 & 23.25 \\
South & 16 & 23.25 \\
West & 31 & 23.25
\end{tabular}

$$
\chi^{2}=6.694
$$

Degrees of freedom $=3$

Probability $=0.08$

\section{Table 10.12}

Nest site direction and a goodness-of-fit test to test for non-random directions: two directions.

\begin{tabular}{l|cc} 
Direction & Observed & Expected \\
\hline North-west & 58 & 46.5 \\
South-east & 35 & 46.5
\end{tabular}

$$
\chi^{2}=6.059
$$

Degrees of freedom $=1$

Probability $=0.01$ 
There are two possible explanations for this phenomenon:

1. yellowheads prefer north-west facing nest sites.

2. suitable sites are more common on the north-west sides of trees. The strongest winds in the Eglinton Valley come from the north-west so it is likely that more knot holes would form on the north-west sides of trees when branches were broken in strong winds.

I have already demonstrated that yellowheads are not selective in their use of nests, and the second explanation seems more likely.

\subsubsection{Nest hole entrance size}

More nest holes with entrances with minimum dimensions of about $5 \mathrm{~cm}$ were used than were nest holes with larger or smaller entrances (Figure 10.1). Low use of small holes might reflect the difficulty of entering such holes; the minimum size used was $2.5 \mathrm{~cm}$. Low use of larger holes could be an adaptation to exclude large predators. While these arguments suggest that yellowheads might be selectively using such nest holes, selectivity cannot be conclusively demonstrated without information on the abundance of holes of all sizes, and I have already shown that amongst the holes yellowheads use they show no selectivity.

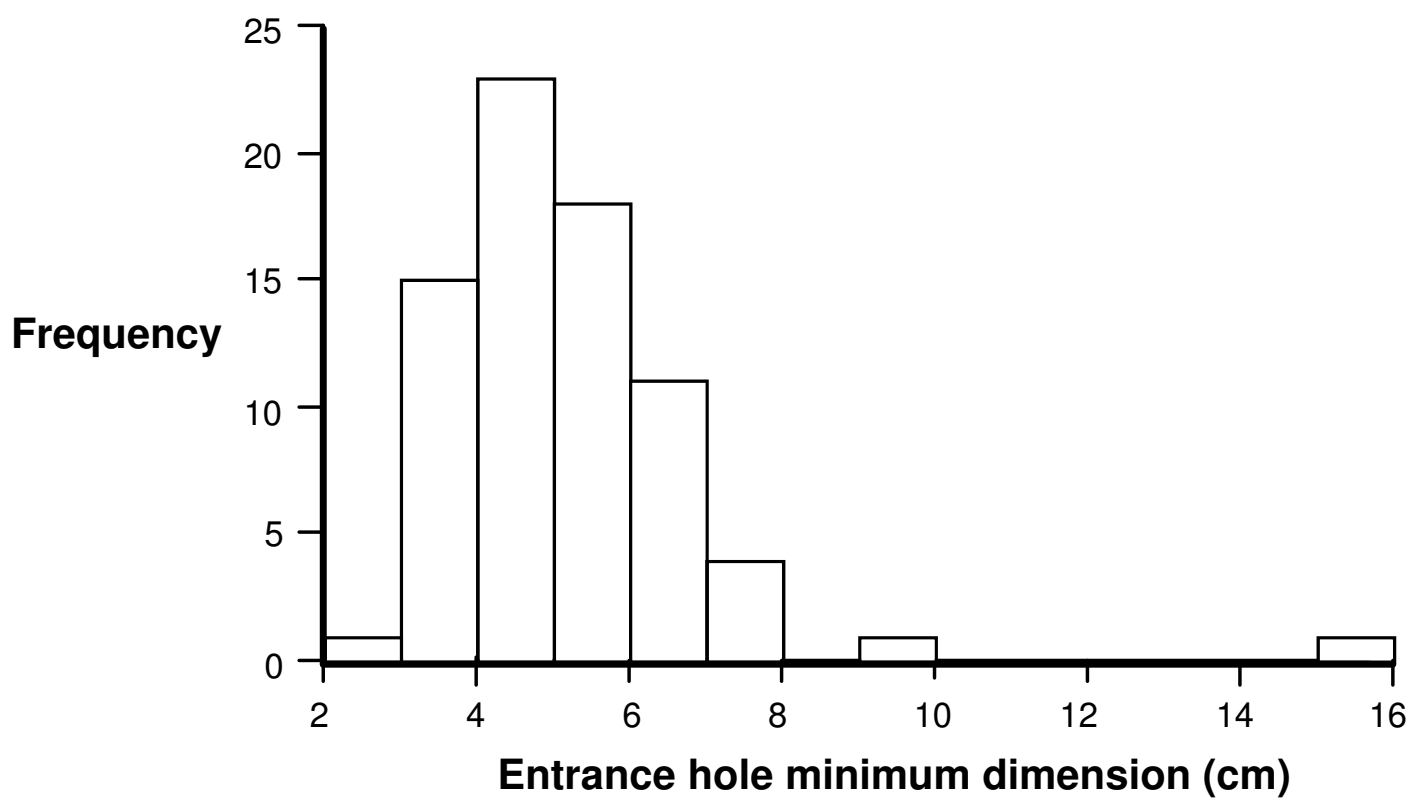

Figure 10.1: $\quad$ Frequency distribution of nest hole entrance size. 


\section{DISCUSSION}

\subsubsection{Nest site selectivity}

This study indicates that neither previous successful use, prior knowledge, nor inherent quality make a nest site more likely to be used by yellowheads; they show no detectable nest site selectivity. Clearly yellowheads must be sufficiently selective to chose between totally unsuitable sites and those that are usable, but my evidence suggests that this is the limit of their selectivity.

Many hole nesting species show well developed patterns of nest site selectivity (e.g., Nilsson 1984, Belles-Iles and Picman 1986 and Lumsden 1986), though often such patterns result from competition between species for nest sites, rather than the innate preferences of the species. Competition can not only result in patterns of nest use that appear to indicate preference, but it can be a significant force in the development of nest site preference.

The finding that yellowheads show little nest site selectivity supports Soper's (1976) suggestion that hole nesting is a recently acquired trait, and also suggests that there is little competition for nest sites.

The suggestion that hole nesting is a recently acquired trait is not profound. The yellowhead probably differentiated from its nearest relatives, the whitehead and brown creeper, as recently as the Pleistocene (Fleming 1982), and neither these species nor any of their more distant relatives, the pachycephaline warblers (Sibley and Ahlquist 1987), are hole nesters. If the phylogeny is correct, then yellowheads have hole nested for less than 10,000 years.

\subsubsection{Nest sites and habitat preferences}

Yellowheads are probably not selective in their use of nest sites, but nest sites are not equally abundant in all types of tree, so yellowheads nest more frequently in some types of tree than others. Yellowheads are selective in their use of forest (Chapter 8) and the availability of nest sites could well be a factor that has influenced their habitat preferences. To determine whether yellowhead nesting habits have any effect on their habitat preferences, we need to ask the following question:

Do the forest types that yellowheads prefer contain trees with high numbers of nest sites?

In Chapter 8 I investigated the relationships between the distribution of yellowheads and vegetation and topography. This study involved measuring the vegetation in 413 plots 
and determining whether or not yellowheads were present nearby. I used this same data in answering the above question.

To determine whether there is any association between habitat preferences and the availability of nest sites I cross classified each of the tree types (species and size of tree) according to:

1. Whether or not that tree type was more common in vegetation plots from which yellowheads were recorded than it was in vegetation plots without yellowheads.

2. Whether or not that tree type contained more nest sites than expected on the basis of surface area (see section 10.3.4.2).

Contingency table analysis was used to test for association (Table 10.13).

\section{Table 10.13}

Contingency table analysis for association between habitat preference and nest site availability.

\begin{tabular}{ll|cc} 
& & \multicolumn{2}{|c}{ Abundance in preferred forest } \\
& & Less common & More common \\
\hline & & 5 & 5 \\
Use as & Frequent & 3 & 6 \\
nest site & Infrequent &
\end{tabular}

$$
\begin{aligned}
\chi^{2}= & 0.54 \\
\text { Degrees of freedom }= & 1 \\
\text { Probability }= & 0.4625
\end{aligned}
$$

This admittedly weak test indicates that nest site abundance is probably not important in habitat selection.

\subsubsection{Evolution of hole nesting}

The most widely accepted evolutionary explanation of hole nesting (Lack 1968, Alerstam and Högstedt 1981) suggests that hole nesting is an adaptation to reduce predation, particularly by avian predators. The choice between hole nesting and not hole nesting is a trade off between the benefits of reduced predation and the costs of increased competition for nest sites. Thus one would expect hole nesting to evolve when there is significant avian predation, and little competition for hole nests. Chapter 5 indicated that yellowheads suffer significant predation (and parasitism) from avian predators, it remains only to assess the degree of competition for nest sites. 
Competition for nest sites has two components: nest site density, and the number of competitors.

\subsubsection{Nest site density}

The analogy between nest site re-use and mark recapture studies (see section 10.3.3.3) suggests that mark-recapture analysis may be an appropriate way to estimate the total number of nest sites available.

Caughley (1977) noted that ". . . mark-recapture analyses are not particularly robust and small deviations from their implicit assumptions can produce large errors in the results." He further said that the greatest source of error is unequal catchability and that its causes can be grouped into three main categories:

1. a property inherent in the individual (expressed in its behaviour in the immediate vicinity of a capturing device).

2. the result of learning (animals may become capture-prone or capture shy), and

3. a property depending on relative opportunity of capture (an animal cannot be trapped if no trap is placed within its home range).

Though I have already demonstrated empirically that nests are probably "equally catchable" i.e., randomly used (section 10.3.3 ), the novelty of this analysis is such that a detailed examination of theoretical nest "catchability" is warranted.

In nest site terms the first category includes variations in the likelihood of nest sites being found by yellowheads and variations in the likelihood of yellowheads choosing to use them once found. In mark-recapture studies animals that cannot be caught confound results, whereas nest sites that yellowheads cannot find or will not use are of no interest. Estimates of the total number of nest sites will in fact be estimates of the total number of usable nest sites; an entirely appropriate bias.

In nest site terms learning would occur when yellowheads preferentially chose the same nest time after time ("trap-happy" in mark recapture terms), or when they consistently avoided re-using nests ("trap-shy"). The analysis in section 10.3.3.1 has already shown that yellowheads neither preferentially use nor avoid nests they already know about.

The third source of unequal catchability is probably less of a problem in this nest site study than in most mark-recapture ones. Yellowheads are territorial and build nests anywhere within their territories, I have found several nests very near territory boundaries. In my study area all the available forest was occupied so that all nest sites could have been used, and to this extent the catchability of nests is equalised. However, nest sites that occur in territories with few nest sites, will be more catchable that those 
in territories where there are many. This is closely analogous to the situation where animals are trapped in traps that can only catch one at a time. Animals that live in an area where there are many other animals are less likely to be caught than animals living where there are few.

Overall it seems that from a theoretical point of view equal catchability is probably less of a problem in studying yellowhead nest sites than it is in most mark-recapture studies.

The acceptance of equal catchability means that it is appropriate to use mark-recapture estimates of the "population size" of nest sites. I have used two methods to estimate the number of nest sites available (Table 10.14). Eberhardt's (1969) method estimates the number of nest sites that were never used from the parameters of the Poisson distribution. This estimate is based on the assumption that their has been no "immigration" or "emigration" of nest sites. This is clearly not the case: new nest sites are formed as the trees grow and old nest sites are lost when trees fall or when nest sites in rotten wood collapse. However, the rates of such "immigration" and "emigration" must be very low. Bailey's (1952) method is a simple modification of the Lincoln index (Caughley 1977) and offers the improvement in that it also assumes no immigration, but it allows for emigration so long as the rates of emigration of marked and unmarked nests are the same. Standard errors can be estimated for the Bailey estimate. 


\section{Table 10.14}

Nest use and re-use and estimates of the number of nest sites.

\section{Year}

\begin{tabular}{llll}
$84-5$ & $85-6$ & $86-7$ & $87-8$ \\
\hline
\end{tabular}

Nest sites

New nest sites (unmarked)

Used sites (recaptures)

Bailey's (1952) method

Estimated no. of nest sites

Standard error of estimate

Eberhardt's (1969) method

Estimated no. of nest sites $=307$

$\begin{array}{rrrr}23 & 23 & 29 & 18 \\ 23 & 21 & 24 & 13 \\ 0 & 2 & 5 & 5\end{array}$

215

$\begin{array}{rrrr}- & 184 & 220 & 215 \\ - & 86 & 74 & 67\end{array}$

The variability of these estimates means that they cannot be regarded as accurate estimates of the number of nest sites available. However, this analysis clearly demonstrates that there are many more suitable nest sites available than are used by yellowheads.

\subsubsection{Competition for nest sites}

Other hole nesting species that occur in the Eglinton Valley that might use similar-sized holes include, starlings (Sturnus vulgaris), kingfishers (Halcyon sancta), and yellowcrowned parakeets (Cyanoramphus auriceps).

Kingfishers and starlings will have little impact on yellowheads because their numbers are low (only 1 pair of kingfishers and about 5 pairs of starlings nested in my study area). Yellow-crowned parakeets are about as numerous as yellowheads and use some of the same nest sites (three parakeet nests were found in sites previously used by yellowheads). Significant competition for nest sites is however unlikely because there are about 10 times as many nest sites as the yellowheads need and because parakeet breeding is spread throughout the year, and they use a wider range of hole types (pers. obs.).

It seems unlikely that either inter or intra-specific competition for nest sites limit the density or productivity of yellowheads in my study area. In pre-human times 
yellowheads may also have had to compete with red-crowned parakeets (Cyanoramphus novaezelandiae) for nests, but they would not have had to compete with starlings.

The presence of large numbers of nest holes and relatively little competition for them lends support to Alerstam and Högstedt's (1981) model of the evolution of hole nesting behaviour.

The fact that neither of the yellowheads closest relatives have adopted hole nesting suggests that holes are more abundant in yellowhead habitat than they are in either whitehead or brown creeper habitat. Of the three species the yellowheads seems most specialised in its habitat requirements, being almost confined to tall beech forests. The other two are found in a range of forest types including scrub and exotic forests. Some forest types occupied by whiteheads and brown creepers consist of trees too small to contain holes large enough for the birds to nest in, whereas tall beech forests invariably have large numbers of nest holes. 


\section{CHAPTER 11}

\section{DISCUSSION}

The 13 questions posed in the introduction (section 1.6) have been addressed in the preceding 6 chapters, it remains only to evaluate the successes and limitations of this study, determine whether its several parts combine to provide a coherent explanation for the decline of yellowheads, and make recommendations for future research and management.

\section{1}

\section{SUCCESSES}

This study has provided considerable information on many aspects of yellowhead biology that were previously unknown or incorrectly reported. They include, length of breeding season, clutch size, productivity, territorial behaviour and territory and mate fidelity. While investigating these aspects of yellowhead biology, tree climbing and mist netting techniques for use in tall beech forest had to be developed; yellowheads had not been banded before I started this study and none had been caught this century.

One important discovery of this study was the fact that yellowhead sexes were easily distinguishable by their calls and behaviour. They had previously been sexed by plumage, which is unreliable.

Before this study the relationship between beech mast and mouse and stoat numbers had been well documented (King 1983), but the effect of stoat plagues on forest birds had not been conclusively demonstrated. This study demonstrates that stoat plagues have considerable effects on the demography of yellowheads and perhaps other hole nesting forest birds.

Modelling of yellowhead populations has provided new insights into their long-term prospects for survival and is a technique not previously used on threatened birds in New Zealand.

In my investigations of the relationship between long-tailed cuckoos and yellowheads I assessed the effect of cuckoos on yellowhead productivity, found that hole nesting probably protects yellowheads from cuckoo predation and gathered much new information about cuckoo breeding. 
My investigation of yellowhead habitat preferences was more detailed and rigorous than previous studies of habitat preference in New Zealand, and my investigation of relationships between bird distribution and fertility seems to be a new area of exploration. The development of habitat suitability indices is widely used by wildlife managers and scientists in Australia and the United States, and I hope my use of it in this study will introduce this useful technique to New Zealanders.

By developing a new vegetation measurement technique (phiangle) I have overcome some of the problems of detecting selective habitat use in surface feeding birds, and my habitat use study has demonstrated that yellowheads are probably specialist tall forest birds.

In investigating patterns of nest site selection I explored some novel ways of estimating nest site abundance and assessing nest site selectivity, and demonstrated that nest site availability probably has little effect on the yellowheads habitat preferences.

\section{2}

\section{LIMITATIONS}

Many of the limitations of this study result from the behaviour of yellowheads. They spend most of their time high in trees where they are difficult to see and catch. Their nests, which are high off the ground, are difficult to find and dangerous to monitor.

When I started the study I had hoped to devote a proportion of my time to investigating co-operative breeding in yellowheads. The variable incidence of co-operative breeding between years, the difficulties of banding yellowheads, and the high over-winter mortality of immature birds, prevented investigation of relationships between kinship and co-operative breeding and the effect of co-operative breeding on yellowhead breeding success. Yellowheads proved to be a very poor subject for a co-operative breeding study.

This study might perhaps have resolved the contradictory views of Soper (1976), who said that yellowhead sexes could be distinguished by plumage, and Cunningham and Holdaway (1986) who said they could not. Resolution of this problem would have required repeated capture of many yellowheads throughout the year. Yellowheads were difficult enough to catch during their breeding season when they could be caught in mist nets erected near their nests, but in the winter they proved almost impossible to catch. Furthermore, they quickly became unresponsive to taped calls which were used to attract them into nets, and after one capture were difficult to catch again. These problems might have been worth overcoming if I had not discovered that yellowheads 
were easily sexed by their calls and behaviour; sorting out the plumage-sex relationship became rather a waste of time.

A decision not to attempt to assess stoat and rodent densities was made at the beginning of the study in an attempt to reduce the workload and because stoats had already been monitored in the Eglinton Valley for several years. In hindsight monitoring rat and stoat numbers may have been desirable.

Mathematical models are by definition limited. The greatest limitation of the models of yellowhead population changes came from the small data base from which vital rates were estimated, lack of knowledge of the variability of the timing and amplitude of stoat plagues, and lack of knowledge of the nature of density dependence in yellowheads. These problems could only be addressed in a much longer study.

An obvious limitation of this study was the fact that it was carried out mostly in summer. The behaviour and habitat relationships of yellowheads in winter may be of considerable consequence to the conservation of the species. However, given that I could not spend the whole year in the field, the ecology of yellowheads in the summer was likely to be more revealing than that in the winter. Yellowheads were most vulnerable to predation during their breeding season, and while breeding they did not wander as widely as they did at other times; their patterns of habitat selection were thus more obvious.

\section{3}

\section{SYNTHESIS}

The following is a synthesis of those aspects of yellowhead biology that have a bearing on the species decline.

During the Pleistocene when yellowheads, whiteheads and brown creepers probably differentiated (Fleming 1982), competition caused specialisation of the yellowhead for tall forest, and the brown creeper for scrub and low forest (Fleming 1982). In the North Island the whitehead faced no competition from close relatives and it is more of a generalist than either of the species which competed in the South Island. Evidence of the yellowhead's specialisation includes its rather specific habitat preferences for forests containing large trees (Chapter 8 and 9), and the fact that yellowhead fledglings remain dependent on their parents for a long time (Chapter 3).

The yellowhead, whitehead and brown creeper all suffer brood parasitism by long-tailed cuckoos, and from evidence presented here I suggest that in response to parasitism and 
predation yellowheads recently became hole nesters (Chapter 7). Hole nesting was probably not an option for whiteheads and brown creepers, because they often inhabit scrub or low forest with few holes, whereas yellowheads live in tall forest with numerous holes.

Living in tall forest and nesting in holes in trees probably served the yellowhead well for several thousand years. The Maori, who arrived about 1200 years ago, destroyed some tall forest, but much remained. The predators they introduced, dogs (Canis familiaris), and kiore (Rattus exulans) were not very arboreal and had little effect on yellowheads. At the beginning of European settlement yellowheads were still common in forest throughout the South and Stewart Islands.

Unfortunately hole nesting, while effective against avian and ground dwelling predators provides no protection against arboreal mammalian ones. Arboreal predators introduced by Europeans in the 1800's, such as ship rats and stoats found nesting yellowheads easy prey. In addition to hole nesting two features of yellowhead behaviour made them more susceptible to predators than some other forests birds: only the female incubates, and the timing of their nesting coincides with peak stoat densities (King and Moody 1982a, 1982b, Chapters 5 and 6).

In diverse lowland mixed forests, such as in Central Westland and Stewart Island, there are high numbers of rats and their numbers (along with stoats in Westland) increased every summer; yellowheads quickly disappeared. In beech forests, stoat numbers were high only once every 4-6 years after a beech mast, and rat numbers never high. Yellowheads have survived 100 years of stoat and rat predation in these forests.

Yellowheads appear to have two strategies for breeding and holding territories. Some birds remain in the vicinity of their territories all year round and raise two broods, whereas others establish new territories at the beginning of each breeding season, raise only one brood and leave their territories at the end of the breeding season. At Knobs Flat most pairs adopted the former strategy, whereas at Plato Creek and Arthur's Pass (Read 1987) they followed the latter (Chapter 5).

Choice of strategies is probably related to habitat quality. Yellowheads living in tall valley floor forest that I identified as "good habitat," seem to employ the "two-brood" strategy, whereas as those in "poor habitat" employ the "one-brood" strategy. Good habitat occurred on more fertile sites than poor habitat (Chapter 8) and it seems likely that good habitats are more productive and have a greater abundance of invertebrate food than poor ones. In areas with few invertebrates, food supplies may be depleted 
during the raising of the first brood. Thus females may not be in good enough condition to lay another clutch (see Laet and Dhondt 1989), and furthermore, there may be too few invertebrates to feed the first brood or to raise a second. Thus they raise only one brood and leave their territories soon after the first brood fledges.

In good yellowhead habitats there will be high numbers of invertebrates. During the raising of the first brood the food supply may not become unduly depleted, and the birds can raise a second brood and even stay on their territories throughout the year.

The productivity of yellowheads that raise two broods in good habitats is sufficient to match losses from stoat plagues every 4-6 years, whereas the productivity of yellowheads that raise one brood in poor habitats, is not (Chapters 5 and 6). In good habitats yellowheads have remained common and can probably survive long-term. In poor habitats they have slowly declined, and can only be expected to survive where they are in close proximity to a large productive population from which they receive immigrants.

The relationships between predation, productivity and habitat quality explain why yellowheads have disappeared from most of the settled parts of the South Island. Tall forests at low altitudes on valley floors were usually the first to be cleared for farmland, and the remaining patches of forest were often not of high enough quality to continue to support yellowhead populations in the face of predation.

It does not, however, explain the almost total disappearance of yellowheads from the northern South Island, since there are still some high quality, tall beech forests there, which ought to support highly productive yellowhead populations (Chapter 8).

I suggested in the Introduction that vespulid wasps were strongly implicated in the yellowhead's decline, and by eliminating a likely competing explanation (habitat quality), this study further implicates them. It also provides some indirect evidence of the wasp's role in the yellowhead's decline.

Most of the recent yellowhead records from the northern South Island come from high altitude silver beech forest; there are few recent records from tall beech forests on fertile valley floors. From the relationships between habitat quality and yellowhead productivity revealed in this study, one would expect yellowheads to disappear first from the high altitude sites and last from the tall forests on fertile valley floors. Competition between wasps and yellowheads provides a possible explanation for this apparent anomaly. In the northern South Island wasps reach highest densities in beech 
forests with large concentrations of Ultracoelostoma assimile, a scale insect that excretes sugary honeydew on which wasps feed. Beech scale insect does not live on silver beech and its density declines with altitude; it is rare above $800 \mathrm{~m}$ (Crozier 1981). Thus wasps are uncommon in high altitude silver beech forests, and yellowheads in such forests probably suffer little competition from them.

Another reason for the yellowhead's vulnerability to wasp competition is the timing of their breeding and the long period of dependence of their young. Most New Zealand forest passerines breed much earlier than yellowheads (Chapter 5) and their young are dependent on them for much shorter times (Chapter 3). Most have finished breeding and their young are fully independent by the time wasp numbers reach their peak in March and April (Sandlant and Moller 1989). In contrast the last yellowhead nests fledge in March and all young are still dependent on their parents during March and April. High wasps numbers coincide with the vulnerable juvenile stage in the life-cycle of yellowheads.

Though German wasps have been in the South Island for about 30 years the recent arrival of common wasps poses a possible increased threat to yellowheads. Following the arrival of common wasps in the northern South Island there has been a net increase in the density of wasps in beech forests (Sandlant and Moller 1989), and common wasps are more tolerant of colder conditions and may spread south into forests that have no wasps at present.

It is argued in this study that predation, particularly by stoats, can significantly lower yellowhead productivity. In beech forests, predator numbers are only high after beech masts, but even in these forests the productivity of populations in only the most favoured sites is high enough to match losses to predators. In areas where predator numbers fluctuate less, such as in mixed forests, predation may have caused the local extinction of yellowheads. Thus yellowheads may now be particularly vulnerable to any factor that further reduces their productivity.

Other forest-dwelling insectivorous birds, such as grey warblers, robins, brown creepers, tits and fantails, may not have suffered so greatly from introduced predators because they are not hole nesters. Their productivity has remained sufficiently high to withstand competition from introduced wasps.

King $(1984,1985)$ argued that stoats now have little effect on the densities of surviving forest birds, and that control of stoats is justified only in a few cases as part of integrated attempts to save very rare birds. This study, however, suggests that stoat 
predation may have permanently reduced all yellowhead populations, caused some to go into long term declines from which they will eventually become extinct, and has perhaps made the species vulnerable to competition from wasps. Other hole nesting forest birds may be affected by stoats in a similar manner, and in this respect it may be significant that two other rare but widespread forest birds, kaka (Nestor meridionalis) and yellow-crowned parakeet, are also hole nesters.

\section{4}

\section{FUTURE RESEARCH}

\subsubsection{Research for management}

Identifying stoats as an important factor in the decline of yellowheads does not necessarily mean that stoat control can help the species. Attempts to control stoats on a large scale using existing techniques may make little difference to yellowhead populations or be prohibitively expensive. The top priority for future work on yellowheads must be to determine whether cost-effective stoat control techniques can be developed that reduce stoat numbers sufficiently to have a beneficial effect on yellowhead populations.

Wasps are still only implicated in the decline of yellowheads. Interactions between wasps and yellowheads will probably prove impossible to investigate directly, since wasps and yellowheads do not occur together in significant numbers. However, investigation of the diet of wasps and yellowheads in similar forest types may give an indication of the extent of competition between the two.

Many of the conclusions regarding the effect of stoats on yellowhead populations are based on the population models presented in Chapter 6. Models are simplistic representations of the "real world" and in this case were based on only 4 years data. Conservation policy makers are faced with the choice of financing further research to refine yellowhead population models, or financing yellowhead research and management based on what they already know. To refine population models will require research for many more years, since stoat plagues occur infrequently, yet if the existing models are approximately correct more yellowhead populations will have become extinct in the meantime.

My suggestions for future management orientated research in priority order are:

1. Develop cost-effective stoat control techniques that reduce predation of yellowhead nests to sustainable levels. 
2. Assess the degree of competition between yellowheads and wasps.

3. Monitor yellowhead populations to provide better estimates of yellowhead population parameters and enable refinement of population models.

\subsubsection{Theoretical research}

There are some interesting features of yellowhead behaviour and ecology still little understood. More work could be done on their social organisation and the evolutionary forces that maintain co-operative breeding. Mechanisms for holding territories and the factors that determine which of the two breeding and territory-holding strategies are adopted could also usefully be investigated. The relationships between long-tailed cuckoos, yellowheads and brown creeper is of interest, particularly with respect to the evolution of hole nesting. Possible competition between brown creepers and yellowheads is also of interest.

However, because of the difficulties of observing yellowheads and the fact they are confined to remote forest blocks far from research institutions, they are not good subjects for esoteric studies of ecology and behaviour. Apart from hole nesting, most of the likely research topics could equally well be looked at using brown creepers or whiteheads, which are more common and easily accessible. Future research on yellowheads is likely to be driven by the requirements of conservation managers, rather than the curiosity of scientists.

\section{I1.5 IMPLICATIONS FOR YELLOWHEAD MANAGEMENT}

Though some research is required before we know whether stoats can be controlled to a level that will enable the long-term survival of some small low-productivity yellowhead populations, some populations are so small that they will have to be managed without this knowledge or they will become extinct. The yellowhead populations on Mt Stokes in the Marlborough Sounds, and in and around Arthur's Pass National Park, are not only very small and vulnerable, but they are of great significance since they are the northernmost known populations. Attempts should be made to control stoats in these areas immediately.

Stoat control for yellowheads living in beech forest need only be carried out during years when stoat numbers are high, since predation by stoats in other years may be almost negligible. In most forests high stoat numbers are predictable, since they occur after a beech mast and an increase in mouse numbers. Monitoring of beech seedfall and 
mouse numbers should be an integral part of any attempt to control stoats for the benefit of yellowheads.

This study indicates that in the absence of wasps "two-brood" yellowhead populations can probably recover from the effects of repeated stoat plagues. However, if several stoat plagues were to occur in quick succession, then the smaller "two-brood" populations as well as "one-brood" ones would be vulnerable. Furthermore the spread of common wasps into areas previously uninhabited by wasps is a cause for concern. To enable the necessary quick reaction to any decline in the apparently stable yellowhead populations in the southern South Island, a selection of these populations should be monitored regularly.

Though yellowheads are vulnerable they are still in sufficiently high densities in places for some experimental management to be carried out. Techniques for keeping and raising yellowheads in captivity, and for transferring birds to predator free islands should be investigated. 


\section{ACKNOWLEDGEMENTS}

Thanks to:

Ben Bell who supervised this study.

Ben Bell, Rod Hitchmough, Henrik Moller, Ralph Powlesland and Kath Walker who criticised earlier drafts of this thesis.

Alan and Connie Wright and Darren Peters who provided needed company during my occasional visits to civilisation (Te Anau).

The 80 Operation Raleigh venturers who collected most of the data for my study of habitat preferences.

Colin O'Donnell and Peter Dilks who provided information on yellowheads at Arthur's Pass.

Murray and Bette Elliott for lending me their car for about a year.

The Wildlife Service, Department of Internal Affairs and the Department of Lands and Survey who paid for the first 18 months of this study.

The Department of Lands and Survey who awarded me a National Parks and Reserves Scholarship to continue the study when the earlier finance ran out.

The Department of Conservation who continued to pay the scholarship when the Department of Lands and Survey was disestablished in 1987.

The Royal Forest and Bird Protection Society and the Ornithological Society of New Zealand who provided finance when I was between government departments.

Kath Walker who made as great a financial contribution as any of the government departments, and a much greater emotional one. 


\section{REFERENCES}

AIROLA, D.A.; BARRETT, R.H. 1985. Foraging and habitat relationships of insect-gleaning birds in a Sierra Nevada mixed-conifer forest. The Condor 87: 205-216.

ALERSTAM, T.; HÖGSTEDT, G. 1981. Evolution of hole-nesting in birds. Ornis Scandinavica 12: 188-193

BAILEY, N.T.J. 1952. Improvements in the interpretation of recapture data. Journal of Animal Ecology 21:120-127.

BATCHELER, C.L.; CRAIB, D.G. 1985. A variable area plot method of assessment of forest condition and trend. New Zealand Journal of Ecology 8: 83-95.

BECKING, J.H.; SNOW, D.W. 1985. Brood parasitism. Pages 67-70 in Campbell, B.; Lack, E. (editors). A Dictionary of Birds. T \& AD Poyser, Calton.

BELLES-ILES, J.; PICMAN, J. 1986. Nesting losses and nest site preferences in house wrens. The Condor 88: 483-486.

BERRY, K. 1986. Introduction: Development, testing and application of wildlife-habitat models. Pages 3-4 in Verner, J.L.; Morrison, M.L.; Ralph, C.J. (editors) Wildlife 2000. University of Wisconsin Press, Wisconsin.

BOYCE, M.S. 1977. Population growth with stochastic fluctuations in the life table. Theoretical Populations Biology 12: 366-373.

BRADLEY, D.W. 1985. The effects of visibility bias on time-budget estimates of niche breadth and overlap. The Auk 102: 493-499.

BRENNAN, L.A.; BLOCK, W.M.; GUTIERREZ, R.J. 1986. The use of multivariate statistics for developing habitat suitability index models. Pages 177-182 in Verner, J.L.; Morrison, M.L.; Ralph, C.J. (editors) Wildlife 2000. University of Wisconsin Press, Wisconsin.

BROWN, J.H.; KODRIC-BROWN, A. 1977. Turnover rates in insular biogeography: Effect of immigration on extinction. Ecology 58: 445-449.

BROWN, J.L.; BROWN, E.R. 1980. Reciprocal aid-giving in a communal bird. Zeitschrift Tierpsychologie 53: 313-324.

BUCKINGHAM, R. 1982. Distribution of yellowhead (Mohoua ochrocephala) in the Catlins State Forest Park. Unpublished report to the New Zealand Forest Service, Invercargill.

BULL, P.C.; GAZE, P.D.; ROBERTSON, C.J.R. 1978. Bird Distribution in New Zealand A Provisional Atlas 1969-1976. Ornithological Society of New Zealand, Wellington.

CAUGHLEY, G. 1977. Analysis of Vertebrate Populations. John Wiley and Sons, London.

COKER, P.M. 1980. Wildlife values of the forested areas of Hokonui and Catlins. Fauna Survey Unit Report No. 23. Wildlife Service, Department of Internal Affairs, Wellington. 
COKER, P.M.; IMBODEN, C. 1979. Wildlife values and wildlife conservation in South Westland. Fauna Survey Unit Report No. 21. New Zealand Wildlife Service, Department of Internal Affairs, Wellington.

CRAWLEY, M.C. 1981. Wildlife Service Research Priorities. Unpublished report, New Zealand Wildlife Service, Department of Internal Affairs, Wellington.

CROZIER, L.R. 1981. Beech honeydew: forest produce. New Zealand Journal of Forestry 26: 200-209.

CUNNINGHAM, J.B. 1985. Breeding ecology, social organization and communicatory behaviour of the brown creeper (Finschia novaeseelandiae). PhD thesis, University of Canterbury, Christchurch.

CUNNINGHAM, J.B.; HOLDAWAY, R.N. 1986. Morphology and head colour in the yellowhead. Notornis 33: 33-36.

CUNNINGHAM, J.M. 1949. Rearing of long-tailed cuckoo. New Zealand Bird Notes 3: 176178.

CUNNINGHAM, J.M. 1985. Long-tailed cuckoo. Page 255 in Robertson, C.J.R. Reader's Digest Complete Book of New Zealand Birds. Reader's Digest, Sydney.

DANIEL, M.J. 1976. Population ecology of ship and Norway rats in New Zealand. Pages 145152 in Dingwall, P.R.; Atkinson, I.A.E.; Hay, C. (editors). The Ecology and Control of Rodents in New Zealand Nature Reserves. Information Series, No. 4. Department of Lands and Survey, Wellington.

DAVIES, N.B.; LUNDBERG, A. 1985. The influence of food on time budgets and the timing of breeding in the dunnock, Prunella modularis. Ibis 127: 100-110.

DE LAET, J.F.; DHONDT, A.A. 1989. Weight loss of the female during the first brood as a factor influencing second brood initiation in Great Tits Parus major and Blue Tits $P$. caeruleus. Ibis 131: 281-289.

DENNISON, M.D.; ROBERTSON, H.A.; CROUCHLEY, D. 1984. Breeding of the Chatham Island warbler (Gerygone albofrontata). Notornis 31: 97-105.

DOW, D.D. 1980. Communally breeding Australian birds with an analysis of distributional and environmental factors. Ети 80: 121-140.

EBERHARDT, L.L. 1969. Population estimates from recapture frequencies. Journal of Wildlife Management 33:28-39.

ELLIOTT, G.; O'DONNELL, C. 1988. Recent decline in yellowhead populations. Science and Research Internal Report No. 29. Department of Conservation, Wellington.

ELLIOTT, G.P. 1986. Mohua a declining species. Forest and Bird 17: 26-28.

ELLIOTT, G.P.; OGLE, C.C. 1985. Wildlife and wildlife habitat values of Waitutu Forest Western Southland. Fauna Survey Unit Report No. 39. New Zealand Wildlife Service, Department of Internal Affairs, Wellington. 
EMLEN, S.T. 1982. The evolution of helping. II. The role of behavioural conflict. American Naturalist 119: 40-53.

EMLEN, S.T. 1984. Co-operative breeding in birds and mammals. Pages 305-339 in Krebs, J.R.; Davies, N.B. (editors). Behavioural Ecology. An Evolutionary Approach. Second edition. Blackwell, Oxford.

EMLEN, S.T.; VEHRENCAMP, S.L. 1983. Cooperative breeding strategies among birds. Pages 93-120 in Brush, A.H.; Clark, G.A. (editors). Perspectives in Ornithology. Cambridge University Press, Cambridge.

FALLA, R.A.; SIBSON, R.B.; TURBOTT, E.G. 1979. The New Guide to the Birds of New Zealand. Colins, Auckland.

FLACK, J.A.D. 1976. New Zealand robins. Wildlife - A Review 7: 15-19.

FLEMING, C.A. 1982. George Edward Lodge The Unpublished New Zealand Bird Paintings. Nova Pacifica, Wellington.

FORDHAM, R.A. 1961. Notes of the German wasp Vespula germanica. Tuatara 9:24-31.

FORDHAM, R.A. 1962. Spread of the German wasp in New Zealand. Tuatara 9: 129-130.

FRITH, H.J. (editor). 1976. Reader's Digest Complete Book of Australian Birds. Reader's Digest, Sydney.

FULTON, R. 1904. The kohoperoa or koekoea, long-tailed cuckoo (Urodynamis taitensis) : An account of its habits, description of a nest containing its (supposed) egg, and a suggestion as to how the parasitic habit in birds has become established. Transactions and Proceedings of the New Zealand Institute 36: 113-148.

GAZE, P.D. 1978. Breeding biology of the North Island rifleman. Notornis 25: 244.

GAZE, P.D. 1982. Yellowhead study. OSNZ News 25: 7.

GAZE, P.D. 1985. Distribution of yellowheads (Mohoua ochrocephala) in New Zealand. Notornis 32: 261-269.

GILL, B.J. 1982. Breeding of the grey warbler Gerygone igata at Kaikoura, New Zealand. Ibis 124: 123-147.

GILL, B.J. 1983. Brood-parasitism by the shining cuckoo Chrysococcyx lucidus at Kaikoura, New Zealand. Ibis 125: 40-55.

GILL, B.J.; McLEAN, I.G. 1986. Morphometrics of the whitehead Mohoua albicilla on Little Barrier Island, New Zealand. New Zealand Journal of Zoology 13: 267-271.

GILL, B.J.; POWLESLAND, R.G.; POWLESLAND, M.H. 1983. Laying seasons of three insectivorous song-birds at Kowhai Bush, Kaikoura. Notornis 30: 81-85.

GOLDSTEIN, R.A. 1977. Reality and models: Difficulties associated with applying general ecological models to specific situations. Pages 207-215 in Solomon, D.L.; Walters, C.F. (editors). Mathematical models in biological discovery. Springer-Verlag, New York. 
GRAY, R.S. 1969. Breeding biology of rifleman at Dunedin. Notornis 16: 5-22.

GROENENDAIL, VAN, J.; KROON, DE, H. 1988. Projection matrices in population biology. Trends in Ecology and Evolution 3: 264-269.

GURR, L. 1947. Measuring bird dimensions. Notornis 2: 57-61.

GUTHRIE-SMITH, H. 1936 Sorrows and joys of a New Zealand naturalist. A.H. and A.W. Reed, Dunedin.

HARPER, G. 1988. Sonographic analysis of the vocalisations of the yellowhead (Mohoua ochrocephala). Unpublished BSc(hons) thesis, Victoria University of Wellington, Wellington.

HARRELL, F.E. Jr. 1983. The logist procedure. Pages 181-202 in Joyner, S.P. (editor). SUGI supplementary library user's guide. SAS Institute Incorporated, Cary, North Carolina.

HARRISON, C.J.O. 1969. Helpers at the nest in Australian passerine birds. Еmu 69: 30-40.

HARVEY, P.H.; GREENWOOD, P.J.; PERRINS, C.M. 1979. Breeding area fidelity of great tits. Journal of Animal Ecology 48: 305-313.

HERLUGSON, C.J. 1983. Nest site selection in mountain bluebirds. The Condor 83: 252-255.

HOLDAWAY, R.N. 1988. The New Zealand passerine list: What if Sibley and Ahlquist are right? Notornis 35: 63-70.

HOLMES, R.T.; ROBINSON, S.K. 1981. Tree species preferences of foraging insectivorous birds in a northern hardwoods forest. Oecologia 48: 31-55.

HUMPHREY, P.S.; BRIDGE, D.; LOVEJOY, T.E. 1968. A technique for mist netting in the forest canopy. Bird Banding 39: 43-50.

HUMPHREY, P.S.; PARKES, K.C. 1959. An approach to the study of molts and plumages. Auk 76: 1-31.

KEARTON, P.M. 1979. Breeding biology and behaviour of the yellow-breasted tit. Unpublished MSc thesis, University of Canterbury.

KING, C.M. 1983. The relationships between beech (Nothofagus sp.) seedfall and populations of mice (Mus musculus), and the demographic and dietary responses of stoats (Mustela erminea), in three New Zealand forests. Journal of Animal Ecology 52: 141-66.

KING, C.M. 1984. Immigrant Killers. Oxford University Press, Auckland.

KING, C.M. 1985. Stoat in the dock. Forest and Bird 16(3): 7-9.

KING, C.M.; MOODY, J.E. 1982a. The biology of the stoat (Mustela erminea) in the National Parks of New Zealand. III. Morphometric variation in relation to growth, geographical distribution, and colonisation. New Zealand Journal of Zoology 9: 81-102.

KING, C.M.; MOODY, J.E. 1982b. The biology of the stoat (Mustela erminea) in the National Parks of New Zealand. IV. Reproduction. New Zealand Journal of Zoology 9: 103118. 
KINSKY. F.C. (convener). 1970. Annotated Checklist of the Birds of New Zealand. Reed, Wellington.

LACK, D. 1948. The significance of clutch size. III. Some interspecific comparisons. Ibis 90: 25-45.

LACK, D. 1968. Ecological Adaptations for Breeding in Birds. Methuen, London.

LEFKOVITCH, L.P. 1965. The study of population growth in organisms grouped by stages. Biometrics 21: 1-18.

LESLIE, P.H. 1945. On the use of matrices in certain population mathematics. Biometrika 33: 183-212.

LOTKA, A.J. 1907. Relation between birth rates and death rates. Science 26: 21-22.

LOVEGROVE, T.G. 1985. Page 136 in Classified Summarised Notes. Notornis 32.

LUMSDEN, H.G. 1986. Choice of nest boxes by tree swallows, Tachycineta bicolor, house wrens, Troglodytes aedon, eastern bluebirds, Sialia sialis and European starlings, Sturnus vulgaris. Canadian Field Naturalist 100: 343-349.

MACHLIS, L.; DODD, P.W.D.; FENTRESS, J.C. 1985. The pooling fallacy: problems arising when individuals contribute more than one observation to the data set. Zeitschrift Für Tierpsychologie 68: 201-214.

MARK, A.F. 1977. Vegetation of Mount Aspiring National Park New Zealand. National Parks Scientific Series Number 2. National Parks Authority, Wellington.

McLEAN, I.G. 1982. Whitehead breeding, and parasitism by long-tailed cuckoos. Notornis 29: $156-158$.

McLEAN, I.G. 1984. Breeding by fantails (Rhipidura fuliginosa) on Tiritiri Island. Notornis 31: $279-283$.

McLEAN, I.G. 1985. Breeding status of cuckoos on offshore islands of New Zealand: some corrections. New Zealand Journal of Ecology 8: 123-124.

McLEAN, I.G. 1987. Response to a dangerous enemy: should a brood parasite be mobbed. Ethology 75: 235-245.

McLEAN, I.G. 1988. Breeding behaviour of the long-tailed cuckoo on Little Barrier Island. Notornis 35: 89-98.

McLEAN, I.G.; DEAN, S.; de HAMEL, R. 1987. Co-operative breeding in brown creeper? Notornis 34: 223.

McLEAN, I.G.; GILL, B.J. 1988. Breeding of an island-endemic bird: the New Zealand whitehead Mohoua albicilla; Pachycephalinae. Emu 88: 177-182.

McLEAN, I.G.; GILL, B.J.; CURTHOYS, L.P. 1986. Mortality, interference and injury at whitehead nests. Notornis 33: 266-268. 
McLEAN, I.G.; JENKINS, P.F. 1980. Breeding and development of the New Zealand fantail. Notornis 27: 105-133.

McLEAN, I.G.; WAAS, J.R. 1987. Do cuckoo chicks mimic the begging calls of their hosts? Animal Behaviour 35: 1896-1907.

McSWEENEY, G. 1987. The fate of western Southland forests. Forest and Bird 18: 20-21.

MONTGOMERY, N.R. 1977. Single Rope Techniques a guide for vertical cavers. Sydney Speleological Society, Sydney.

MOORS, P.J. 1978. Methods for studying predators and their effects on forest birds. pp 47-57 in Dingwall, P.R.; Atkinson, I.A.E.; Hay, C. (editors). The ecology and control of rodents in New Zealand nature reserves. Information Series No 4. Department of Lands and Survey, Wellington.

MOORS, P.J. 1983. Predation by mustelids and rodents on the eggs and chicks of native and introduced birds in Kowhai Bush, New Zealand. Ibis 125: 137-154.

MORRISON, M.L. 1984. Influence of sample size and sampling design on analysis of avian foraging behaviour. The Condor 86: 146-150.

MORSE, D.H. 1980. Behavioural Mechanisms in Ecology. Harvard University Press, Massachusetts.

NILSSON, S.G. 1984. The evolution of nest-site selection among hole-nesting birds: the importance of nest predation and competition. Ornis Scandinavica 15: 167-175.

NILSSON, S.G. 1986. Evolution of hole-nesting in birds: on balancing selection pressures. Auk 103:432-435.

NOSKE, R.A. 1985. Habitat use by three bark-foragers of eucalyptus forests. Pages 193-204 in Keast, A.; Recher, H.F.; Ford, H.; Saunders, D. (editors). Birds of Eucalyptus Forests and Woodlands. Royal Australasian Ornithologists Union and Surrey Beatty and Sons, Chipping Norton, New South Wales.

O'DONNELL, C.F.J.; DILKS, P. 1988. A method for quantifying habitat use by forest birds. Science and Research Series No. 4. Department of Conservation, Wellington.

OLIVER, H.C. 1968. Annotated index to some early New Zealand bird literature. Wildlife Publication No. 106. Department of Internal Affairs, Wellington.

OLIVER, W.R.B. 1955. New Zealand Birds (second edition). A.H. and A.W. Reed, Wellington.

PASCOE, J. 1957. Mr. Explorer Douglas. Reed, Wellington.

PAYNE, R.B. 1985. Cuckoo. Pages 123-126 in Campbell, B.; Lack, E. (editors). A Dictionary of Birds. T \& AD Poyser, Calton.

PAYNE, R.B.; PAYNE, L.L.; ROWLEY, I. 1985. Splendid wren Malurus splendens response to cuckoos: an experimental test of social organization in a communal bird. Behaviour 94: 108-127. 
PERRINS, C.M. 1970. The timing of birds breeding seasons. Ibis 112: 242-255.

POWLESLAND, M.H. 1982. A breeding study of the South Island fantail (Rhipidura fuliginosa fuliginosa). Notornis 29: 181-195.

POWLESLAND, R.G. 1983. Breeding and mortality of the South Island robin in Kowhai Bush, Kaikoura. Notornis 30: 265-282.

PRESS, S.J.; WILSON, S. 1978. Choosing between logistic regression and discriminant analysis. Journal of the American Statistical Association 73: 699-705.

READ, A.F. 1984. The abundance and habitat use of yellowheads (Mohoua ochrocephala Gmelin, 1789) in the Hawdon Valley, Arthur's Pass National Park. Unpublished B.Sc. (honours) thesis, University of Otago, Dunedin.

READ, A.F. 1987. The breeding and flocking behaviour of yellowheads at Arthur's Pass National Park. Notornis 34: 11-18.

READ, A.F. 1988a. Habitat use by yellowheads, Mohoua ochrocephala (Aves: Muscicapidae), in the Hawdon River Valley, Arthur's Pass National Park. 1. Habitat preferences. New Zealand Journal of Zoology 15: 461-470.

READ, A.F. 1988b. Habitat use by yellowheads, Mohoua ochrocephala (Aves: Muscicapidae), in the Hawdon River Valley, Arthur's Pass National Park. 2. Time budgets and foraging behaviour. New Zealand Journal of Zoology 15: 471-480.

READ, A.F.; O'DONNELL, C.F.J. 1987. Abundance of yellowheads in the Hawdon River Valley, Arthur's Pass National Park, in 1983 and 1984. Notornis 34: 307-315.

RECHER, H.F.; HOLMES, R.T.; SCHULZ, M.; SHIELDS, J.; DAVANAGH, R. 1985. foraging patterns of breeding birds in eucalypt forest and woodland of southeastern Australia. Australian Journal of Ecology 10: 300-419.

ROBERTSON, H.A.; WHITAKER, A.H.; FITZGERALD, B.M. 1983. Morphometrics of forest birds in the Orongorongo Valley, Wellington, New Zealand. New Zealand Journal of Zoology 10: 87-98.

ROWLEY, I. 1969. Communal species of Australian birds. Bonner Zoologische Beitrage 19: 362-368.

RUSSELL, E.M. 1989. Co-operative breeding - a Gondwanan perspective. Ети 89: 61-62.

SANDLANT, G.R.; MOLLER, H. 1989. Abundance of common and German wasps (Hymenoptera: Vespidae) in the honeydew beech forests of New Zealand in 1987. New Zealand Journal of Zoology 16: 333-343.

SHERLEY, G.H. 1985. The breeding system of the South Island rifleman (Acanthisitta chloris) at Kowhai Bush, Kaikoura, New Zealand. Ph.D. thesis, University of Canterbury, Christchurch.

SIBLEY, C.G.; AHLQUIST, J.E. 1987. The relationships of four species of New Zealand Passerine Birds. Ети 87: 63-66. 
SIBLEY, C.G.; AHLQUIST, J.E. 1985. The phylogeny and classification of the AustraloPapuan passerine birds. Ети 85: 1-14.

SOKAL, R.R.; ROHLF, F.J. 1981. Biometry (second edition). W.H. Freeman and Company, San Francisco.

SONERUD, G.A. 1985. Nest hole shift in Tengmalm's owl Aegolius funereus as defence against nest predation involving long-term memory in the predator. Journal of Animal Ecology 54: 179-192.

SOPER, M.F. 1960. Observations on the nesting of yellowhead. Notornis 8: 259.

SOPER, M.F. 1963. New Zealand Bird Portraits. Whitcomb and Tombs, Christchurch.

SOPER, M.F. 1976. New Zealand Birds (second edition). Whitcoulls, Christchurch.

SPURR, E.B. 1986. Beech management - its effects on bird populations. What's new in forest research No. 146. Forest Research Institute, Rotorua.

THOMAS, D.G. 1974. Some problems associated with the avifauna. In Williams, W.D. (editor). Biogeography and ecology in Tasmania. Junk, The Hague.

TULJAPURKAR, S. 1989. An uncertain life: demography in random environments. Theoretical Population Biology 35: 227-294.

TULJAPURKAR, S. D.; ORZACK, S.H. 1980. Population dynamics in variable environments 1. Long-run growth rates and extinction. Theoretical Population Biology 18: 314-342.

VAN BALEN, J.H.; BOOY, C.J.H.; VAN FRANEKER, J.A.; OSIECK, E.R. 1982. Studies on hole-nesting birds in natural nest sites. I. Availability and occupation of natural nest sites. Ardea 70: 1-24.

VERNER, J.L.; MORRISON, M.L.; RALPH, C.J. (editors). 1986. Wildlife 2000. University of Wisconsin Press, Wisconsin.

VON HAARTMAN, L. 1957. Adaptation in hole nesting birds. Evolution 11:339-347.

WAGNER, J.L. 1981. Visibility bias in avian foraging data. The Condor 83: 263-264.

WARDLE, J.A. 1984. The New Zealand Beeches. New Zealand Forest Service, Wellington.

WHITAKER, A.H. 1972. An improved mist net rig for use in forests. Bird Banding 43: 1-8.

WHITTAKER, R.H.; WOODWELL, G.M. 1967. Surface area relations of woody plants and forest communities. American Journal of Botany 54:931-939.

WILKINSON, A.S.; WILKINSON, A.E. 1952. Kapiti Bird Sanctuary. Stidolph, R.H.D. (editor). Masterton Printing Co., Masterton.

WILLIAMS, G.R. 1962. Extinction of the land and freshwater inhabiting birds of New Zealand. Notornis 10: 15-32.

WILlIAMS, G.R. 1973. Birds. Pages 304 - 333 in Williams, G.R. (editor). The Natural History of New Zealand. Reed, Wellington.

WILLIAMS, M. 1986. Native bird management. Forest and Bird 17: 7-9. 


\section{APPENDIX A}

\section{BASIC DETERMINISTIC POPULATION SIMULATION}

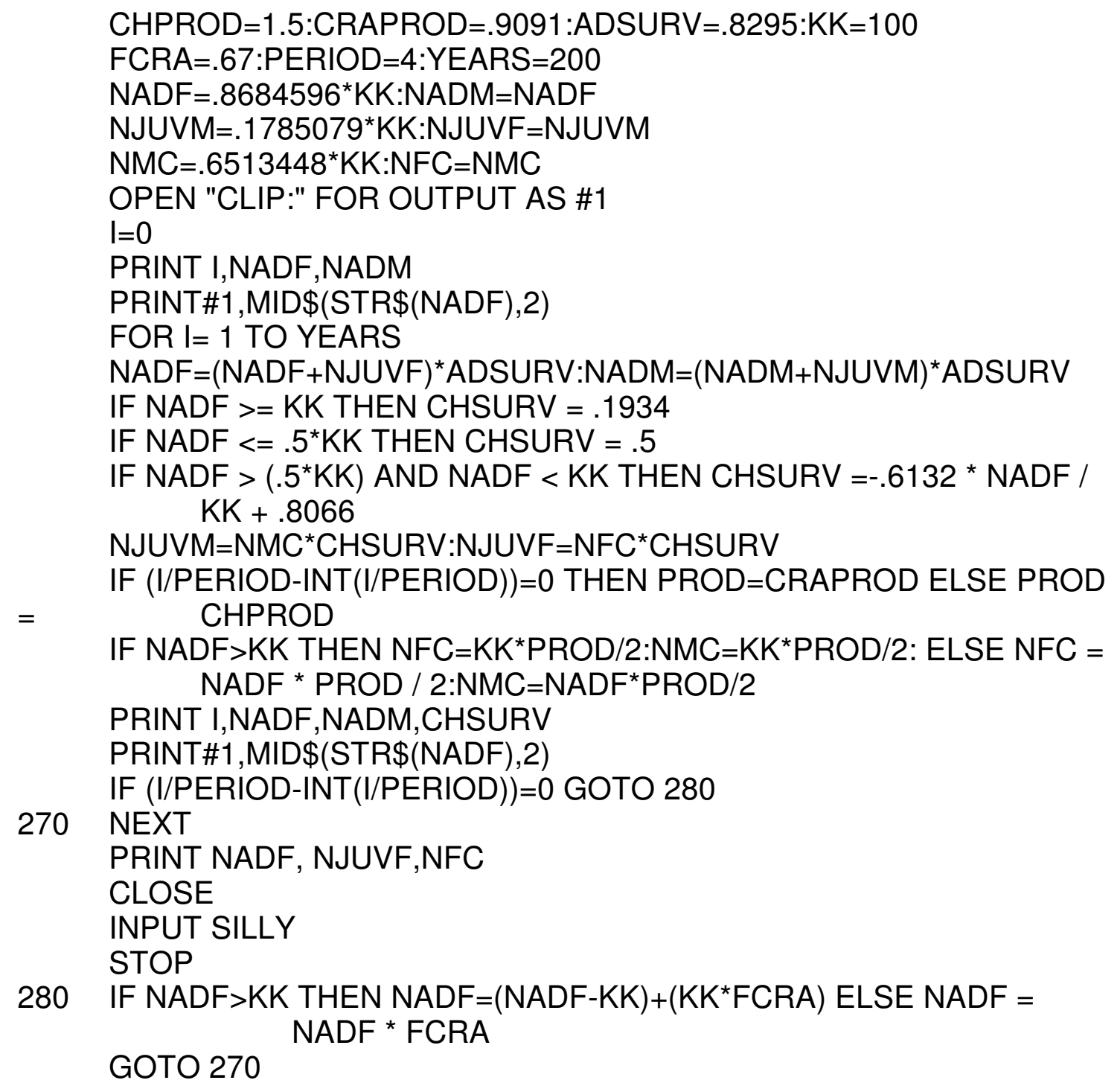




\section{APPENDIX B}

\section{FORTRAN STOCHASTIC POPULATION SIMULATION}

*MAIN PROGRAM

REAL *8 SEED

DIMENSION AVF(100)

CHARACTER ${ }^{*} 1$ TABY

$\mathrm{TABY}=\mathrm{CHAR}(9)$

DO $44 \mathrm{~J}=1,100$

$\operatorname{AVF}(\mathrm{J})=0.0$

44 CONTINUE

OPEN (4, FILE = "TRANS", STATUS = "NEW")

WRITE $\left(9,{ }^{*}\right)$ "TYPE IN A SEED FOR RNG"

READ $(9,120)$ SEED

120 FORMAT (F20.4)

ADSURV $=0.8355$

ADSTD $=0.1566$

WRITE $\left(9,{ }^{*}\right)$ "TYPE IN THE CARRYING CAPACITY"

$\operatorname{READ}(9,121)$ IKK

121 FORMAT (I9)

$\mathrm{FCRA}=0.5$

WRITE $\left(9,{ }^{*}\right)$ "TYPE IN THE PERIOD"

$\operatorname{READ}(9,121)$ IPERIOD

IYEARS $=100$

$P=0$

$P P=0$

ISURV $=0$

IEXT $=0$

IMIN $=100$

$\operatorname{IMAX}=0$

$Y=0$

$Y Y=0$

IYMIN = 100

IYMAX $=0$

DO $500 \mathrm{IJ}=1,100$

INADF $=$ NINT $\left(\mathrm{IKK}^{*} 1.122836\right)$

INADM $=$ INADF

INJUVM $=$ NINT $\left(I^{*} K^{*} 0.2307939\right)$

INJUVF $=$ INJUVM

INMC $=$ NINT $\left(I K K^{*} 1.19335\right)$

INFC $=$ INMC

$\mathrm{I}=1$

ITIMEC $=1$

CALL TTC (IPERIOD,ITIME,SEED)

WHILE $(($ I<=IYEARS $) \cdot A N D \cdot((I N A D F+I N J U V F+I N F C)>0))$

CALL NOADS

$+\quad$ (ADSURV,ADSTD,INJUVF,INJUVM,INADF,INADM,SEED) 
IF (INADF > IKK) THEN

ELSE $\mathrm{INBF}=\mathrm{IKK}$

END IF INBF $=$ INADF

IF (INADM < INBF) INBF = INADM

CALL CHSURVIV (CHSURV, INBF, IKK)

CALL NOJUVS (CHSURV,INFC,INMC,INJUVF,INJUVM,SEED) IF (ITIMEC = ITIME ) THEN

CALL TTC (IPERIOD,ITIME,SEED)

ITIMEC = 0

CALL CRACH (INMC, INFC, INBF,SEED)

ELSE

CALL FEMSURV (FCRA, INADF, INBF,SEED)

END IF

CALL NCRACH (INMC, INFC, INBF,SEED)

$\operatorname{AVF}(I)=\operatorname{AVF}(I)+I N A D F / 100.0$

$\mathrm{I}=\mathrm{I}+1$

REPEAT

ITIMEC = ITIMEC + 1

IF (INADF > IKK) THEN

ELSE $\mathrm{INBF}=\mathrm{IKK}$

END IF

INBF $=$ INADF

IF $($ INADM $<$ INBF) INBF = INADM

$P=P+I N B F$

$\mathrm{PP}=\mathrm{PP}+(\mathrm{INBF} * \mathrm{INBF})$

IF $(\mathrm{I}-1<100)$ THEN

ELSE

$$
\mathrm{IEXT}=\mathrm{IEXT}+1
$$

END IF

ISURV $=$ ISURV +1

IF $($ INBF $>$ IMAX) IMAX = INBF

IF $(\mathrm{INBF}<\mathrm{IMIN}) \quad$ IMIN $=$ INBF

$\mathrm{Y}=\mathrm{Y}+\mathrm{I}$

$Y Y=Y Y+\left(I^{*} \mid\right)$

IF (I>IYMAX) IYMAX = I

IF $($ I $<$ IYMIN $)$ IYMIN = I

WRITE $(9,11)$ IJ,I,INBF

11 FORMAT $(I 3,5 X,|3,5 X| 3$,

500 CONTINUE

WRITE $(9,505)$ IPERIOD,IKK

505 FORMAT ('PERIOD $=$ ',I3,5X,'CARRY CAPACITY = ',I9)

WRITE $(9,501)$ ISURV, IEXT

501 FORMAT ('NO. SURV = ',I3,5X,'NO. EXTINCT = ',I3)

SMEAN $=P / 100.0$

$S D=S Q R T((P P-((P * P) / 100.0)) / 99.0)$

WRITE $(9,503)$ SMEAN,SD

503 FORMAT ('MEAN NO. PAIRS = ',F10.4,5X,'SD = ',F10.4)

WRITE $(9,502)$ IMIN, IMAX 
502 FORMAT ('MIN NO. PAIRS = ',I3,5X,'MAX NO. PAIRS = ',I3)

$S Y=Y / 100.0$

SDY $=\operatorname{SQRT}\left(\left(Y Y-\left(\left(Y^{*} Y\right) / 100.0\right)\right) / 99.0\right)$

WRITE $(9,520)$ SY,SDY

520 FORMAT ('MEAN TIME TO EXT = ',F10.4,5X,'SD = ',F10.4)

WRITE $(9,521)$ IYMIN, IYMAX

521 FORMAT ('MIN TIME TO EXT = ',I3,5X,'MAX = ',I3)

DO $522 \mathrm{I}=1,100$

WRITE $(4,324)$ I,TABY,AVF(I)

324 FORMAT $(I 5, A 1, F 10.4)$

522 CONTINUE

PAUSE

END

${ }^{*}$ CHICK PRODUCTION DURING A CRASH

SUBROUTINE CRACH (INMC, INFC, INBF,SEED)

AVERAGE $=0.9091$

$S T D=0.4255$

CALL NORMAL (AVERAGE,STD,PROD,SEED)

IF $(P R O D<0.0)$ PROD $=0.0$

ICHICKS $=$ ANINT $(P R O D * I N B F)$

IF (ICHICKS > 0) THEN

$\mathrm{PROP}=0.5$

QUOT $=1.0$

IF $\left(0.25^{*} \mathrm{ICHICKS}<3\right)$ THEN

CALL BINOM (PROP , QUOT , ICHICKS , INMC,SEED)

ELSE

CALL BINORM (PROP,ICHICKS,INMC,SEED)

END IF

INFC $=$ ICHICKS - INMC

ELSE

INFC $=0$

INMC $=0$

RETURN

END IF

END

*CHICK PRODUCTION DURING A NORMAL YEAR

SUBROUTINE NCRACH (INMC, INFC, INBF,SEED)

AVERAGE $=2.3867$

$S T D=0.3372$

CALL NORMAL (AVERAGE,STD,PROD,SEED)

IF $(P R O D<0)$ PROD $=0$

ICHICKS $=$ ANINT $\left(\right.$ PROD ${ }^{*}$ INBF $)$

IF (ICHICKS > 0) THEN

$\mathrm{PROP}=0.5$

$\mathrm{QUOT}=1.0$

IF $\left(0.25^{*} \mathrm{ICHICKS}<3\right)$ THEN

CALL BINOM (PROP , QUOT , ICHICKS , INMC,SEED) ELSE 


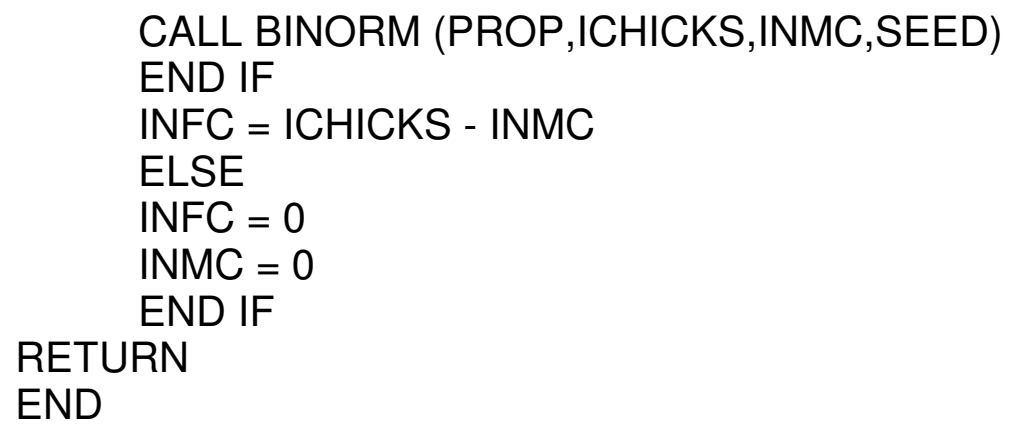

* NUMBER OF ADULT MALES AND FEMALES AT THE BEGINNING OF THE SEASON

SUBROUTINE NOADS (ADSURV, ADSTD, INJUVF, INJUVM, INADF,

$+\quad$ INADM, SEED)

IFSTART $=$ INADF + INJUVF

IMSTART $=$ INADM + INJUVM

1234

CALL NORMAL (ADSURV, ADSTD, SURV, SEED)

IF (SURV < 0.0.OR. SURV >1.0) GOTO 1234

INADF = ANINT(SURV*IFSTART)

RETURN

INADM $=$ ANINT(SURV*IMSTART)

END

*NUMBER OF JUVENILES AT THE BEGINNING OF THE SEASON

SUBROUTINE NOJUVS (CHSURV,INFC,INMC,INJUVF,INJUVM,SEED)

PROP $=1-$ CHSURV

QUOT $=$ CHSURV/PROP

IF (INMC*CHSURV*PROP $<3)$ THEN

ELSE

CALL BINOM (PROP, QUOT, INMC, INJUVM,SEED)

END IF

CALL BINORM (CHSURV,INMC,INJUVM,SEED)

IF (INFC*CHSURV*PROP<3) THEN

ELSE

CALL BINOM (PROP,QUOT,INFC,INJUVF,SEED)

CALL BINORM (CHSURV,INFC,INJUVF,SEED)

RETURN

END IF

END

*FEMALE SURVIVAL DURING A CRASH YEAR

SUBROUTINE FEMSURV (FCRA, INADF, INBF,SEED)

$\mathrm{PROP}=1-\mathrm{FCRA}$

QUOT $=$ FCRA $/$ PROP

INODIE $=$ INADF - INBF

IF (FCRA * PROP*INBF $<3)$ THEN

ELSE

CALL BINOM (PROP, QUOT,INBF, IEND,SEED)

CALL BINORM (FCRA, INBF,IEND, SEED)

END IF

INADF $=$ IEND + INODIE 
RETURN

END

* BINOMIAL-RANDOM VARIATION

SUBROUTINE BINOM (PROP, QUOT, ISTART, IEND,SEED)

IF (ISTART >0) THEN

CALL RANDOM (RAND,SEED)

TEST $=$ PROP $* \star$ ISTART

TESTTOT $=$ TEST

ICOUNT $=1$

IF (RAND $<=$ TESTTOT) THEN

IEND $=0$

ELSE

WHILE (RAND > TESTTOT) ICOUNT $=$ ICOUNT +1

TEST $=$ TEST * QUOT * $(($ ISTART - ICOUNT $)+2)$

$+\quad /($ ICOUNT -1$)$

TESTTOT $=$ TESTTOT + TEST

REPEAT

IEND $=$ ICOUNT -1

END IF

ELSE

IEND $=0$

END IF

RETURN

END

* RANDOM NUMBER GENERATOR

SUBROUTINE RANDOM (RAND,SEED)

REAL*8 SEED,ZGRESS, SEEDY

$X=24298$

$\mathrm{Y}=99991$

$Z=199017$

ZGRESS $=X *$ SEED $+Y$

SEEDY $=((Z G R E S S / Z)-$ INT $(Z G R E S S / Z)){ }^{*} Z$

RAND $=$ SEEDY $/ Z$

SEED $=$ SEEDY

RETURN

END

* TIME TO NEXT CRASH

SUBROUTINE TTC (IPERIOD,ITIME,SEED)

CALL RANDOM (UONE,SEED)

CALL RANDOM (UTWO,SEED)

TSD $=$ IPERIOD $/ 6.0$

ATIME $=$ SQRT $\left(-2^{*} \text { LOG }(\text { UONE })\right)^{*} \mathrm{COS}\left(6.2831853^{*} \mathrm{UTWO}\right)^{*} \mathrm{TSD}$

ITIME = ANINT $($ ATIME $)+$ IPERIOD

IF $($ ITIME < 1) ITIME = 1

RETURN

END 
* NORMAL APPROXIMATION OF BINOMIAL

SUBROUTINE BINORM (AVERAGE, ISTART,IEND, SEED)

START = REAL(ISTART)

CALL RANDOM (UONE,SEED)

CALL RANDOM (UTWO,SEED)

$A=S Q R T\left(-2^{*} \operatorname{LOG}(\mathrm{UONE})\right)^{*} \mathrm{COS}\left(6.2831853^{*} \mathrm{UTWO}\right)$

$A B=A^{*} S Q R T\left(A V E R A G E * S T A R T^{*}(1.0-A V E R A G E)\right)$

IEND $=$ ANINT $\left(A B+\right.$ AVERAGE ${ }^{\star}$ START $)$

IF $($ IEND < 1) IEND = 0

IF (IEND>ISTART) IEND = ISTART

RETURN

END

* DENSITY DEPENDENT CHICK SURVIVAL

SUBROUTINE CHSURVIV (CHSURV, INBF, IKK)

IF (INBF >= IKK) THEN

$$
\text { CHSURV }=0.1934
$$

ELSE

IF (INBF $\left.<=0.5^{*} \mid \mathrm{KK}\right)$ THEN

CHSURV $=0.5$

ELSE

CHSURV $=-0.6132 *$ INBF $/ \mathrm{IKK}+0.8066$ END IF

END IF

RETURN

END

* NORMAL-RANDOM VARIATION

SUBROUTINE NORMAL (AVERAGE,STD,ANSWER,SEED)

CALL RANDOM (UONE,SEED)

CALL RANDOM (UTWO,SEED)

$A=S Q R T\left(-2^{*} \text { LOG }(\text { UONE })\right)^{*} \operatorname{COS}\left(6.2831853^{*}\right.$ UTWO $)$

ANSWER $=\left(A^{*} S T D\right)+$ AVERAGE

RETURN

END 


\section{APPENDIX C}

\section{A NEW METHOD OF MEASURING VEGETATION FOR STUDIES OF BIRD HABITAT USE: POINT HEIGHT INTERCEPT ANALYSIS AT AN ANGLE}

\section{C.1}

INTRODUCTION

In studies of the habitat use of forest birds, patterns of habitat use are often compared with vegetation patterns in order to determine whether birds selectively use some forest components (e.g., Holmes and Robinson 1981, Noske 1985, Recher et al. 1985, Airola and Barret 1985, Read 1988). If birds are not responsive to the vegetation features measured, then such comparisons may fail to detect selective habitat use even though it exists.

Many studies of habitat use measure stem density (e.g. Read 1988) or vegetation cover (e.g. Airola and Barret 1985) because they are provided by well known vegetation mensuration techniques which are easy to use; not because they are parameters which measure features to which birds are likely to be responsive.

Many arboreal insectivorous forest birds take most of their prey from the surface of plants, and it is likely that surface area is a vegetation parameter to which these birds might be responsive. There are, however no documented vegetation mensuration techniques that provide unbiased measures of surface area, without felling trees.

MacArthur \& Horn (1969) and Park (1973) provide methods that measure cover, which is effectively the horizontal surface area of vegetation, but vertical components of forests, such as tree trunks, have little horizontal surface and are virtually unmeasured by these methods.

Here I describe a simple modification of MacArthur and Horn's (1969) method that provides an unbiased method of estimating surface area of vegetation in tall forests. I call the method Point intercept analysis at an angle or "Phiangle". 


\section{C.2.1 Background to the new Method}

MacArthur and Horn's (1969) method involves taking a large number of vertical sightings from the forest floor and recording the height at which the sightings first hit vegetation. For any height interval the cover provided by vegetation is given by:

$$
\text { cover }=\frac{\text { number of sightings that hit vegetation within an interval }}{\text { number of sightings that had not hit vegetation in any lower intervals }}
$$

From this an estimate of the average number of hits per interval is given by:

$$
\text { Average number of hits }=-\log (1-\text { cover })
$$

This estimate assumes that the objects that are being hit are randomly dispersed.

Park's (1973) method is similar but involves actually counting the number of hits in a vertical sighting rather than estimating them. Rather than stopping at the first hit, one "looks round" each hit and thus counts all of them. Park's method has the advantage of counting rather than estimating the hits, but can only be used in relatively low forest; one cannot see round hits more than a few metres up. Furthermore, seeing round hits implies that hits are not sampled along a single line, but within a cylinder. Scott (1965) showed that when cylinders are used the frequency of hits is not directly related to vegetation density, but varies with the size of the cylinder and the size of the object that is hit.

For these reasons I chose MacArthur and Horn's method as the basis for my new method.

The number of hits from Parks method, or the estimated number from MacArthur and Horn's, can be converted directly to "horizontal area surface area density" if the length of the sightings are known. For example, two hits along a five metre sightings implies that there are two square metres of horizontal vegetation surface in five cubic metres of space.

Wilson (1960) working on grassland vegetation showed that sightings inclined at angles of $32.5^{\circ}$ produced measures with least bias in favour of either vertical or horizontal surfaces. My method involves simply taking sightings according to MacArthur and 
Horn's method except that instead of taking the sightings vertically, they are taken at an angle of $32.5^{\circ}$ to the horizontal.

\section{C.2.2 The new method}

Sightings at $32.5^{\circ}$ to horizontal were taken by looking through a camera with a $300 \mathrm{~mm}$ telephoto lens mounted on a tripod. Any vegetation covering a cross marked in the centre of the camera's focussing screen was recorded and its distance from the tripod found by focussing on it and reading the scale on the lens. Height off the ground was calculated as:

$$
\text { height }=\text { distance } \times \sin 32.5^{\circ}
$$

Height was not regarded as being a continuous variable and height measurements were assigned to five metre height classes. To get sightings below the height of the tripod the camera was reversed.

Ideally sightings should be taken from random sites within the area to be sampled, and at random compass bearings. In practice, however, it is easier to sample on a grid pattern at a series of fixed bearings. I was constrained to place the samples in such a way that vegetation could be measured at every grid point. I took sightings at five metre intervals along grid lines in a $50 \mathrm{~m}$ grid (Figure C.1). In theory, only two sightings at $180^{\circ}$ should be taken from one site, since sightings at smaller angles are likely to sample the same tree repeatedly and thus not be independent. As a compromise between this requirement and the problems of getting enough sightings, I took four sightings at $90^{\circ}$ from each site. Using this regime I obtained 80 sightings from each area that I was sampling. MacArthur and Horn recommended that sufficient sightings be taken so that some passed right through the vegetation without hitting. In the tall beech forest in which I sampled 80 was sufficient. 


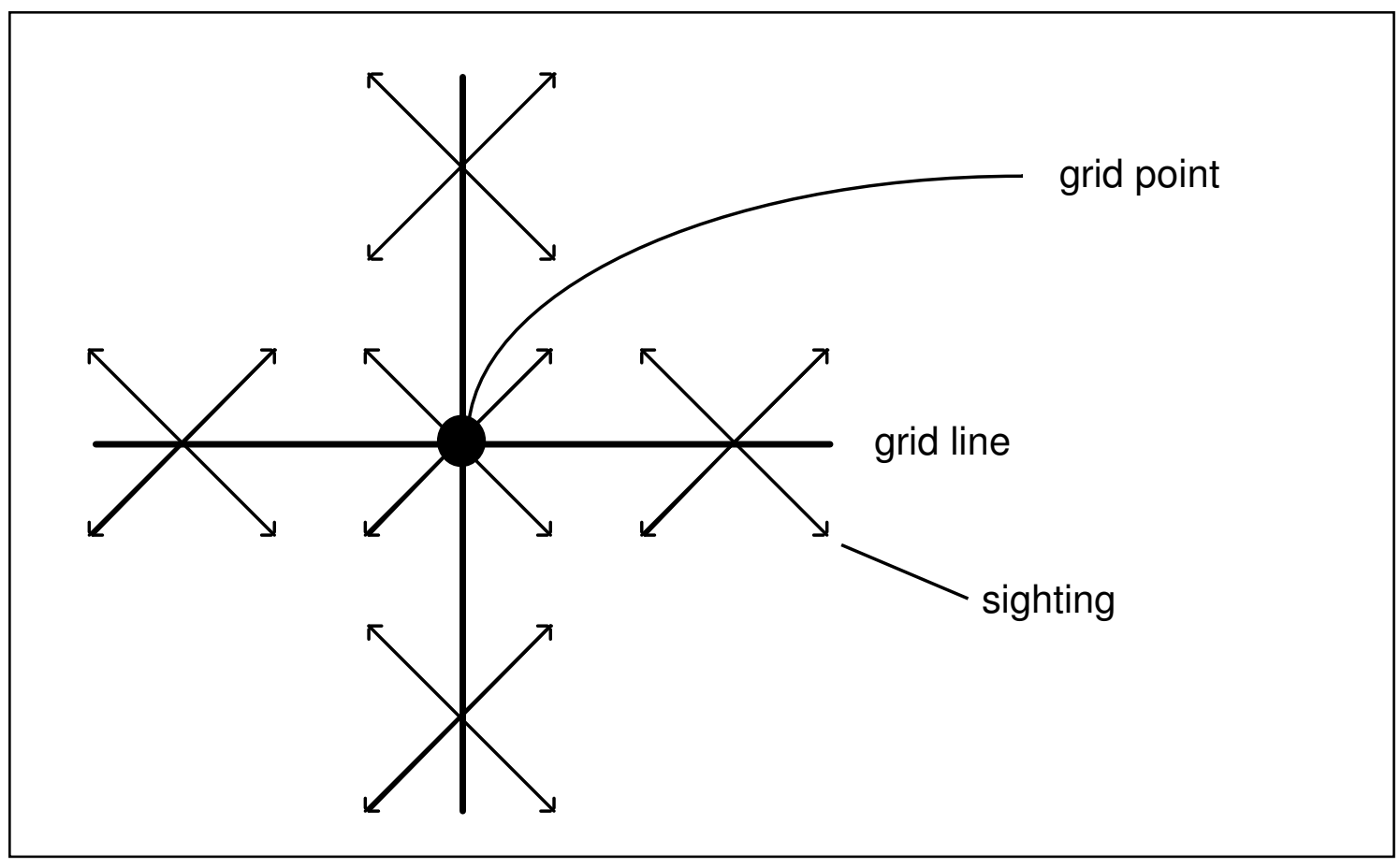

Figure C.1: $\quad$ The arrangement of phiangle sightings with respect to the grid at Knobs Flat.

Because of the $32.5^{\circ}$ angle of the sightings, some hit vegetation far removed from the grid point. Where adjacent grid points are being sampled it is necessary to calculate the horizontal position of the hit on the ground so that hits can be attributed to the correct grid point. Similarly when a sighting passes through a height interval high above the ground without hitting vegetation, the horizontal position of that "miss" has to be calculated. This is easily done using trigonometry. Assuming the sightings are taken on a grid aligned North-South, and given $\mathrm{x}$ and y co-ordinates of the place from which the sightings are taken of are $(0,0)$, the $\mathrm{x}$ and $\mathrm{y}$ co-ordinates of hits and misses are given by:

$$
\begin{aligned}
& \mathrm{x}=\frac{\text { height }}{\tan 32.5^{\circ}} \times \sin (\text { bearing at which sighting was taken }) \\
& \mathrm{y}=\frac{h e i g h t}{\tan 32.5^{\circ}} \times \cos (\text { bearing at which sighting was taken })
\end{aligned}
$$

Having done a series of sightings the cover (at $32.5^{\circ}$ ) in each height interval is calculated as:

$$
\text { cover }=\frac{\text { number of hits in interval }}{\text { number of sightings passing through previous height interval }}
$$


An estimate of the total surface area density (at $32.5^{\circ}$ ) in a height interval is calculated as:

$$
\text { total surface area density }=\frac{-\log (1-\text { cover })}{\text { length of sighting in that interval }}
$$

Surface area density can be apportioned amongst $n$ different objects that are hit as follows:

$$
\text { surface area density of object } t_{i}=\frac{\text { hits }_{\mathrm{i}}}{\sum_{\mathrm{i}=1}^{n} \text { hits }_{\mathrm{i}}} \times \text { total surface area density }
$$

\section{C.3}

\section{REFERENCES}

AIROLA, D.A.; BARRETT, R.H. 1985. Foraging and habitat relationships of insect-gleaning birds in a Sierra Nevada mixed-conifer forest. The Condor 87: 205-216.

HOLMES, R.T.; ROBINSON, S.K. 1981. Tree species preferences of foraging insectivorous birds in a northern hardwoods forest. Oecologia 48: 31-55.

MACARTHUR, R.H.; HORN, H.S. 1969. Foliage profile by vertical measurements. Ecology 50: $802-804$.

NOSKE, R.A. 1985. Habitat use by three bark-foragers of eucalyptus forests. Pages 193-204 in Keast, A.; Recher, H.F.; Ford, H.; Saunders, D. (eds). Birds of Eucalyptus Forests and Woodlands. Royal Australasian Ornithologists Union and Surrey Beatty and Sons, Chipping Norton, New South Wales.

PARK, G.N. 1973. Point height intercept analysis a refinement of point analysis for structural quantification of low arboreal vegetation. New Zealand Journal of Botany 11: 103114.

READ, A.F. 1988. Habitat use by yellowheads, Mohoua ochrocephala (Aves: Muscicapidae), in the Hawdon River Valley, Arthur's Pass National Park. 1. Habitat preferences. New Zealand Journal of Zoology 15: 461-470.

RECHER, H.F.; HOLMES, R.T.; SCHULZ, M.; SHIELDS, J.; DAVANAGH, R. 1985. foraging patterns of breeding birds in eucalypt forest and woodland of southeastern Australia. Australian Journal of Ecology 10: 300-419.

SCOTT, D. 1965. A height frequency method for sampling tussock and shrub vegetation. New Zealand Journal of Botany 3: 253-260.

WILSON, J.W. 1960. Inclined point quadrats. New Phytologist 59: 1-8. 